\title{
Metabolic fate of hallucinogenic NBOMes
}

Sebastian Leth-Petersen, ${ }^{\mathrm{a}, \dagger}$ Charlotte Gabel-Jensen, ${ }^{\mathrm{b}, \dagger}$ Nic Gillings, ${ }^{\mathrm{c}}$ Szabolzs Lehel, ${ }^{\mathrm{c}}$ Hanne D. Hansen, ${ }^{\mathrm{d}}$ Gitte M. Knudsen, ${ }^{\mathrm{d}}$ Jesper L. Kristensen ${ }^{\mathrm{a},{ }^{*}}$

${ }^{a}$ Department of Drug Design and Pharmacology, University of Copenhagen, Denmark.

${ }^{\mathrm{b}}$ Department of Pharmacy, University of Copenhagen, Denmark

${ }^{\mathrm{c}}$ PET and Cyclotron Unit, University Hospital, Copenhagen, Denmark.

${ }^{\mathrm{d}}$ Neurobiology Research Unit and Center for Integrated Molecular Brain Imaging, University Hospital, Copenhagen, Denmark.<smiles>COc1cc(CCNCc2ccccc2OC)c(OC)cc1Br</smiles>

Hallucinogenic<smiles>[R]c1cc(OC)c(CCNCc2ccccc2OC)cc1O[C@@H]1O[C@H](C(=O)O)[C@@H](O)[C@H](O)[C@H]1O</smiles>

\section{Corresponding Author}

* Jesper L. Kristensen, Department of Drug Design and Pharmacology, Faculty of Health and Medical Sciences, University of Copenhagen, Universitetsparken 2, DK-2100 Copenhagen, Denmark. E-mail: jesper.kristensen@ sund.ku.dk. Phone: (+45)35336487.

Table of content:

Microsomal incubations and metabolite identification

P. 1-10

Organic synthesis (entry 1 - 38)

P. 11-32

Radiochemistry

P. 32-36

References

P. 36

Analytical spectra

P.37-117 


\section{Microsomal incubations}

A reaction mixture containing $100 \mathrm{mM}$ phosphate buffer $(\mathrm{pH} 7.4), \mathrm{HLM}$ or PLM $0.5 \mathrm{mg} / \mathrm{ml}$ protein concentration, magnesium chloride $3.3 \mathrm{mM}$, glucose-6-phosphate dehydrogenase $0.4 \mathrm{U} / \mathrm{ml}$, glucose-6phosphate $3.3 \mathrm{mM}$ and $\mathrm{NADP}^{+} 1.2 \mathrm{mM}$ was pre-incubated in a shaking water bath at $37^{\circ} \mathrm{C}$ for $7 \mathrm{~min}$. The reaction was started by adding 1 to a final reaction concentration of $25 \mu \mathrm{M}$. Two identical control incubations with either no liver microsomes or no NADPH regenerating system were included. After $1 \mathrm{~h}$ incubation, the reaction was stopped by mixing with equal volumes of ice-cold acetonitrile. Also time point 0 samples were collected. The samples were stored at $-20{ }^{\circ} \mathrm{C}$ until analysis. Before analysis the thawed samples were centrifuged (14500 rpm for $10 \mathrm{~min}$ ) (Centrifuge 5425, Eppendorf AG, Hamburg, Germany) and the clear supernatant was transferred to LC-vials.

\section{LC-MS $^{n}$}

All analyses were performed using a Thermo-Finnigan Q-Exactive mass-spectrometer (Thermo-Finnigan, San Jose, CA), coupled to a Ultimate 3000 UHPLC-system (Thermo-Finnigan, San Jose, CA). The mass spectrometer was equipped with a heated electrospray ionization source operated in the positive ionisation mode. All compounds of interest was detected as the $[\mathrm{M}+\mathrm{H}]^{+}$adduct. lonization settings were optimized for compound 1 using direct infusion of the synthesized standard into the mobile phase (75 \% A and $25 \% \mathrm{~B}$ ), mobile phase flow rate $0.5 \mathrm{ml} / \mathrm{min}$. The optimized settings were used for all analysis. For MSMS analyses the collision energy (HCD) was set to 35. For full scan experiments the instrument resolution was 70000 in the t-ms ${ }^{2}$ (targeted ms-ms) mode the instrument resolution was 17500.

The column was a Phenomenex Kinetex C18, $2.6 \mu \mathrm{m}, 100 \AA \AA .2 .1 \mathrm{~mm}$ ID $\times 100 \mathrm{~mm}$. The mobile phases for the gradient elution were A: $0.1 \%$ formic acid and either 2 or $5 \%$ acetonitrile in Milli-Q water and B: $0.1 \%$ formic acid and $95 \%$ acetonitrile in Milli-Q water. The gradient was linear running from $100 \% \mathrm{~A}$ and $0 \% \mathrm{~B}$ to $50 \% \mathrm{~A}$ and $50 \% \mathrm{~B}$ over 4 min followed by re-equilibration with $100 \% \mathrm{~A}$ and $0 \% \mathrm{~B}$ for $2 \mathrm{~min}$. The flow rate was $0.5 \mathrm{ml} / \mathrm{min}$ and the column was kept at $50^{\circ} \mathrm{C}$.

For quantitative analysis of 1,6 and 9 in pig plasma, the mass spectrometer was operated in the t-ms ${ }^{2}$ mode including target $\mathrm{m} / \mathrm{z}^{\prime} \mathrm{s} 380.09,366.07$ and 542.10. Quantitation was based on the respective peak areas in extracted ion chromatograms $(\mathrm{m} / \mathrm{z}$ 121.065, $\Delta \mathrm{m} 5 \mathrm{ppm})$ against a standard curve of the synthesized standards spiked in blank plasma. The standards were handled as described for the pig plasma samples; prior to analysis the pig plasma samples $(100 \mu \mathrm{l})$ were precipitated with acetonitrile $(100 \mu \mathrm{l})$. After centrifugation (14500 rpm for $10 \mathrm{~min}$ ) (Centrifuge 5425, Eppendorf AG, Hamburg, Germany) the clear supernatant $(170 \mu \mathrm{l})$ was evaporated to dryness in a vacuum centrifuge at $45^{\circ} \mathrm{C}$ for $2 \mathrm{~h}$ (Concentrator Plus, 
Eppendorf AG, Hamburg, Germany). The dry residue was reconstituted in mobile phase A (70 $\mu \mathrm{l})$, centrifuged (14500 rpm for $10 \mathrm{~min}$ ) (Centrifuge 5425, Eppendorf AG, Hamburg, Germany) and the clear supernatant was transferred to vials for LC-MS analysis. Calibration curves for 1, $\mathbf{6}$ and $\mathbf{9}$ were constructed using the peak area in the extracted ion chromatograms ( $\mathrm{m} / \mathrm{z} 121.065, \Delta \mathrm{m} 5 \mathrm{ppm})$. The calibration curve for 1 and 6 was linear ( $R^{2} 0.995$ and 0.991) covering the concentration range 5-500 nM (1) and 0.5-50 nM (6). The calibration curve for 9 was quadratic $\left(R^{2}\right.$ 0.9998) covering the concentration range 1-100 nM. A standard chromatogram found in figure S1. The results are summarized in figure S2.

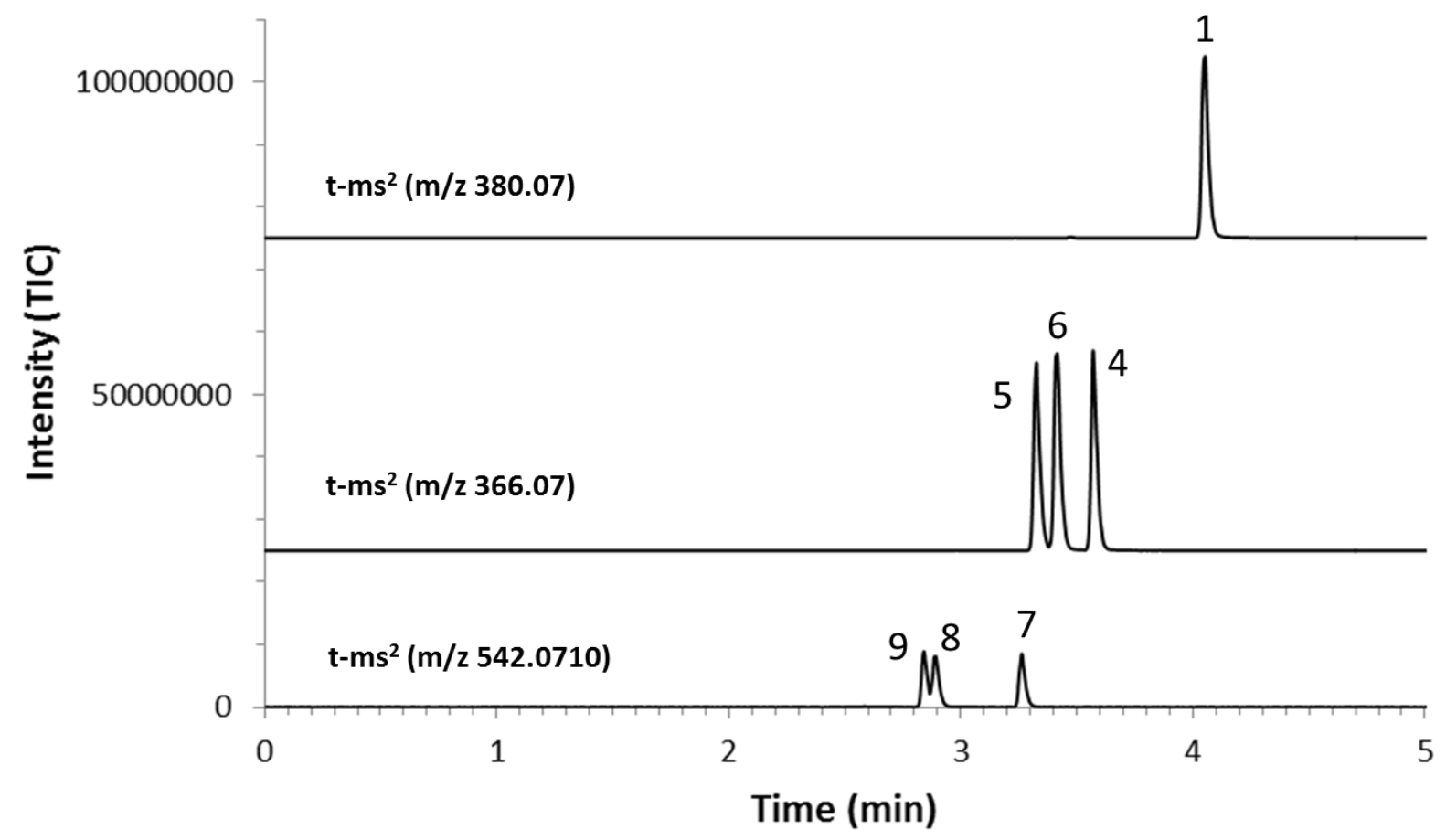

Chromatograms standards in water. Retention time; $1: 4.05 \mathrm{~min}, 4: 3.57 \mathrm{~min}, 5: 3.33 \mathrm{~min}, 6$ : $3,42 \mathrm{~min}, 7: 3.26 \mathrm{~min}, \mathbf{8 :} 2.88 \mathrm{~min}, 9: 2.84 \mathrm{~min}$

Figure S1. 


\begin{tabular}{|c|c|c|c|}
\hline & Glucuronide 9 & Phenol 6 & 25B-NBOMe (1) \\
\hline Time (min) & \multicolumn{3}{|c|}{ concentration (nM) } \\
\hline 2,5 & 14 & 2,38 & 240 \\
\hline 5 & 28 & 1,93 & 128 \\
\hline 10 & 50 & 1,30 & 94 \\
\hline 30 & 87 & 0,63 & 43 \\
\hline 50 & 81 & 0,52 & 31 \\
\hline 70 & 62 & 0,42 & 21 \\
\hline 90 & 45 & 0,32 & 13 \\
\hline 120 & 37 & 0,27 & 9 \\
\hline
\end{tabular}

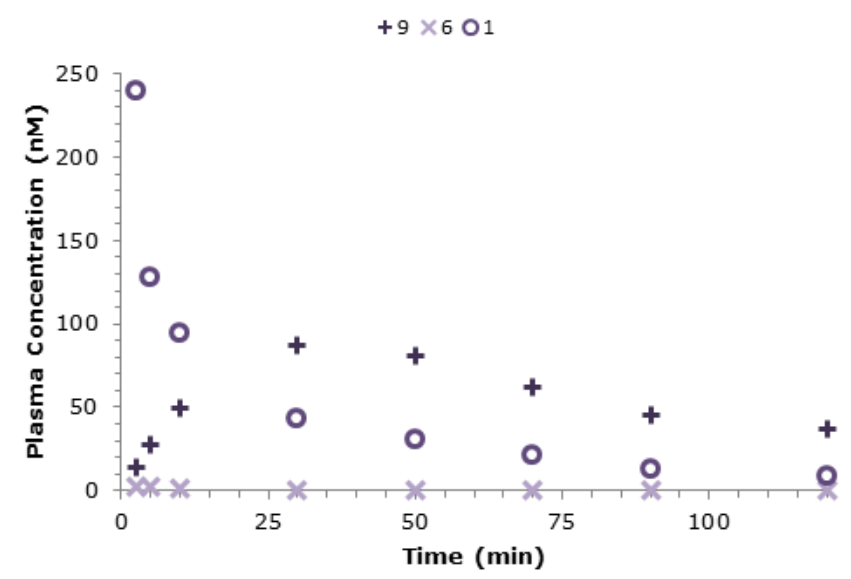

Plasma concentration of 25B-NBOMe (1), phenol 6 and glucuronide 9 in pig over 120 minutes

Figure S2.

\section{Metabolite Identification}

For qualitative analysis the mass spectrometer was operated in the full scan mode $m / z 75-700$. Compounds were assigned as metabolites of $\mathbf{1}$ if the spectrum displayed the typical isotopic pattern of bromine $\left({ }^{79} \mathrm{Br}\right.$ $(50.7 \%)$ and $\left.{ }^{81} \mathrm{Br}(49.3 \%)\right)$. The structure of the detected metabolites was further investigated in $\mathrm{t}-\mathrm{ms}^{2}$ mode including target $\mathrm{m} / \mathrm{z}^{\prime} \mathrm{s} 352.06,366.07,378.08,380.09,382.06 / 384.06,394.08,396.08$, and 542.10. Generally, the collision induced fragmentation resulted in a major fragment ion formed after the cleavage of the bond between the nitrogen and the carbon of the $\mathrm{N}$-benzyl group. This is observed as the major ion with $\mathrm{m} / \mathrm{z} 121.065$ (figure S3, C). When a metabolic demethylation takes place in the N-benzyl ring two major fragment ions are observed in the mass spectrum. The major fragment described above is observed at $m / z 107.049$ along with another major fragment ion at $m / z 243.002$ representing the cleavage of the bond between the nitrogen and the carbon of the phenethylamine moiety (figure S3, A). This is illustrated with representative MS-MS spectra of the synthetized standards of the three phenols $(\mathbf{4}, \mathbf{5}$ and $\mathbf{6}$; figure S3, A and C; entry 14, 15 and 16) and the three glucuronides (7, 8 and 9; figure S3, B and D; entry 20, 21 and 22) 

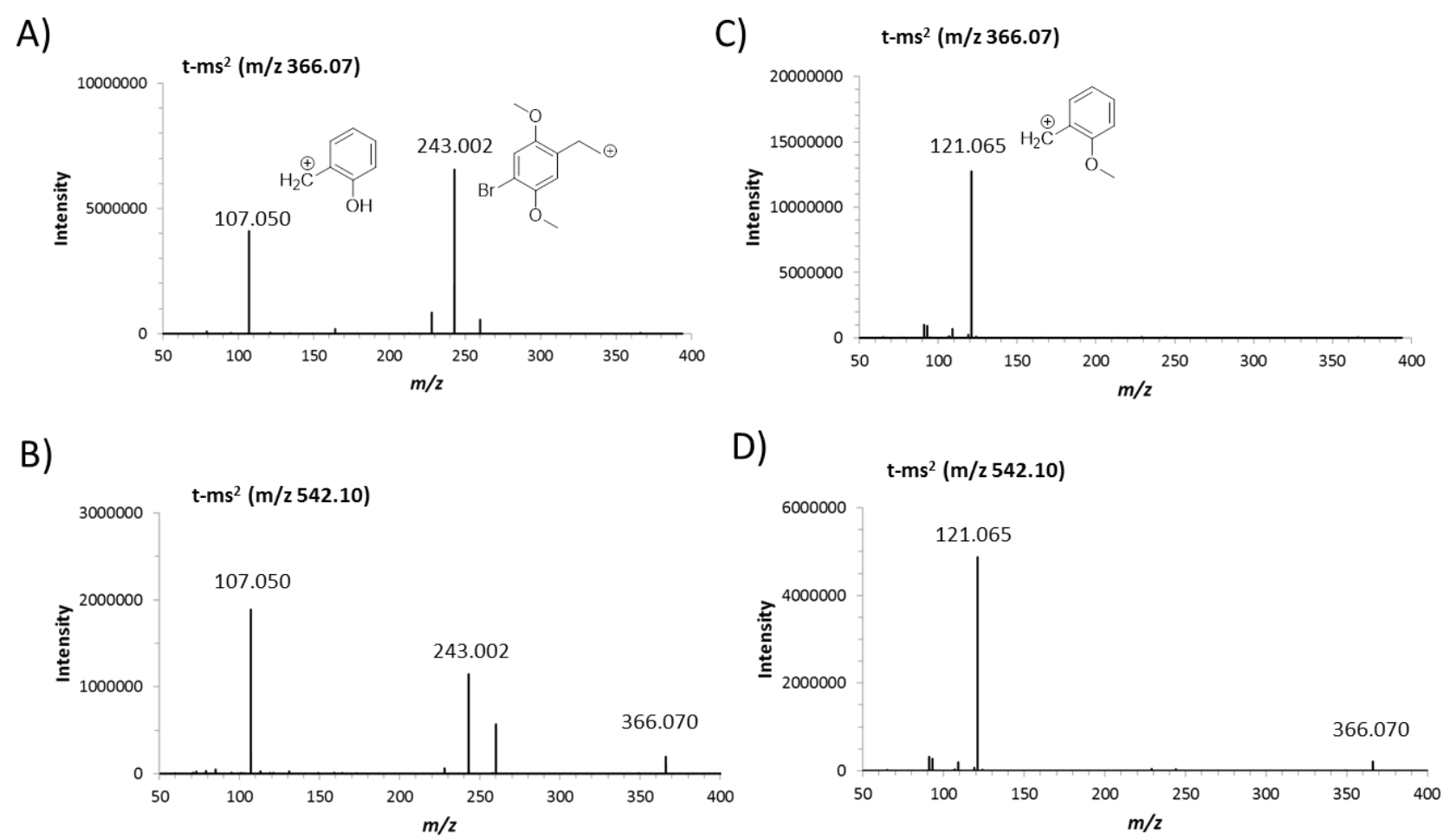

Standard ms-ms spectra. A) phenol 4, B) glucuronide 7, C) phenol 6 and D) glucuronide 9 . The corresponding ms-ms spectra for phenol 5 and glucuronide 8 are similar to phenol 6 and glucuronide $9 \mathrm{~ms}-\mathrm{ms}$ spectra, respectively.

Figure S3.

Metabolic alterations that do not involve demethylation in the $N$-benzyl moiety results in one major fragment corresponding to the $\mathrm{m} / z 121.065$ described above. For example two metabolites (M13 and M15, table S1) with $\mathrm{m} / \mathrm{z}$ of the parent ion $[\mathrm{M}+\mathrm{H}]^{+}=396.084$ corresponding to hydroxylation in the phenethylamine moiety (entry 31 and 34) or in the benzyl moiety (entry 23 and 24) was initially observed in the microsomal incubation experiment. The MS-MS spectra of the metabolites are shown below (figure S4); for M13 the major fragment is observed at $\mathrm{m} / \mathrm{z} 137.060$ corresponding to the $\mathrm{m} / \mathrm{z} 121.065$ fragment with a hydroxylation $(\Delta \mathrm{m}=15.995)$ while the $\mathrm{m} / \mathrm{z} 121.065$ fragment is observed in $\mathrm{M} 15$. Hence, the metabolic hydroxylation in M13 is in the $N$-benzyl moiety, while the hydroxylation is in the phenethylamine moiety in M15. 
A) $\mathrm{M} 13$

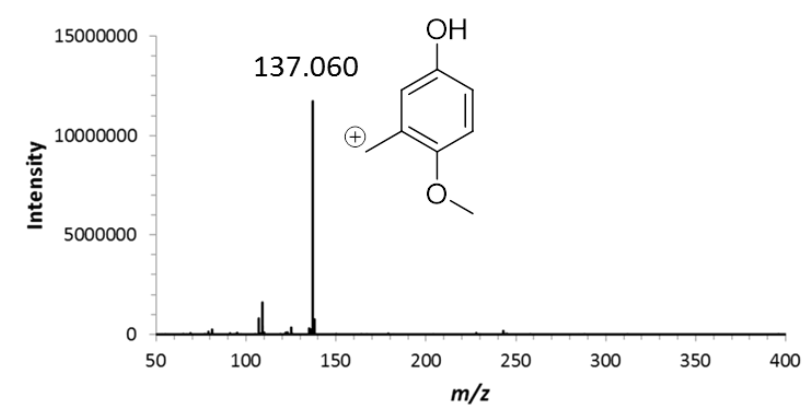

B) M15

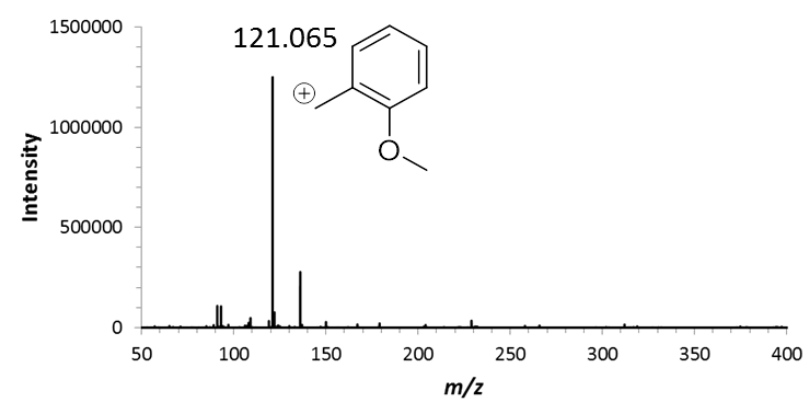

LC t-ms $\mathrm{m}^{2}$ (m/z 396.084) spectra corresponding hydroxylation or N-oxidation. A) M13 (Rt 3.92min) B) M15 (Rt 4.15 $\min )$.

\section{Figure S4.}

Three metabolites (M2, M3 and M5; figure S5) with $\mathrm{m} / \mathrm{z}$ of the parent ion $[\mathrm{M}+\mathrm{H}]^{+}=352.064$ corresponding to two demethylations were initially observed in the microsomal incubation experiment. The structures proposed based on the MS-MS spectra are based on the characteristic fragmentation patterns of phenol 4, 5 and 6 (entry 14, 15 and 16); since the $N$-benzyl moiety is intact (fragment $\mathrm{m} / \mathrm{z} 121.065$ ) in M2, demethylation must be in the phenethylamine moiety. For M5 the $m / z 107.050$ fragment shows that one demethylation is in the $\mathrm{N}$-benzyl moiety, furthermore the other major fragment generally observed when the demethylation is in the $N$-benzyl moiety is observed at $\mathrm{m} / \mathrm{z} 228.987$. This corresponds to the $\mathrm{m} / \mathrm{z}$ 243.002 fragment observed for phenol 4 with a loss of 14.015 which infers metabolic demethylation in both parts of the molecule. The spectra of M3 and M5 are similar suggesting demethylation of one of the methoxy groups in the phenethylamine moiety. Further verification of the structures of M3 and M5 require standards for retention time comparison or verification of metabolite structures by NMR.

\section{A) $M 2$}

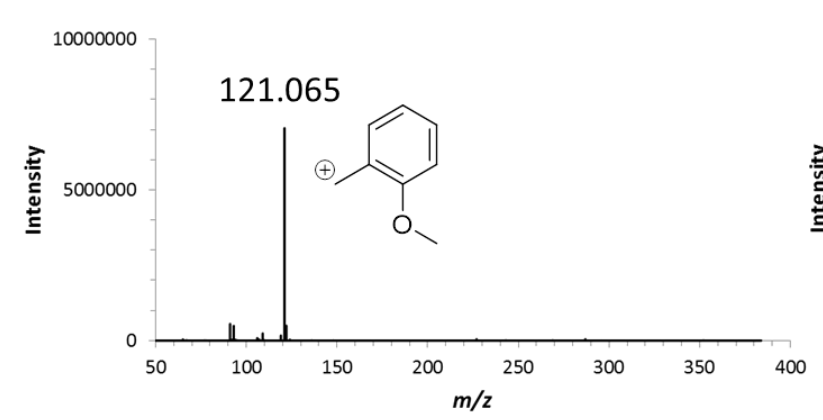

B) M5

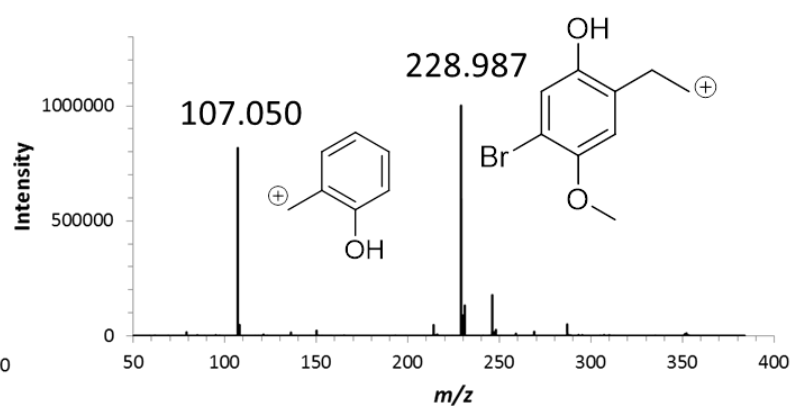

LC $t-m s^{2}$ (m/z 352.08) spectra corresponding to two demethylations. A) M2 (Rt 2.92min) B) M5 (Rt $3.36 \mathrm{~min}$ ).

Figure S5. 
The proposed structures of the metabolites are summarized in table S1 below. The structural assignment is based on deductions similar to the above described for M13/M15 and M2/M5.

The identity of M13 was further verified by retention time matching with synthetic standards. Based on the MS-MS fragmentation pattern (figure S4, A) the hydroxylation is in the $N$-Benzyl ring. Standards hydroxylated in the $\mathrm{N}$-benzyl ring meta and para to the methoxy group were synthesised (entry 24 and 23) and analyzed by LC MS-MS. The gradient was altered to obtain chromatographic separation of the compounds which cannot be differentiated by mass spectrometric fragmentation. The gradient was linear running from $100 \% A$ and $0 \% B$ to $75 \% A$ and $25 \%$ B over 4 min followed by re-equilibration with $100 \% A$ and $0 \% \mathrm{~B}$ for $2 \mathrm{~min}$. Overlay chromatograms from the analysis of the two synthetic standards and the 60 min sample from HLM incubation showed that M13 originates from metabolic hydroxylation in the para position of the $\mathrm{N}$-benzyl ring. Furthermore, it was observed that a metabolite derived from metabolic hydroxylation in the meta position of the $N$-benzyl ring was not detected (figure S6A).

In the same way, the MS-MS fragmentation pattern (table S1) suggests that the hydroxylation in M15 is in the phenethylamine moiety. Beta- and $N$-hydroxylated standards (entry 31 and 34 ) were synthesized and analyzed by LC MS. Overlay chromatograms from the analysis of the beta-hydroxylated standard (entry 31) and the 60 min sample from HLM incubation strongly indicates that this is M15 (figure S6B). Furthermore, the retention time of $\mathrm{N}$-hydroxylated 1 (entry 34) was $4.91 \mathrm{~min}$. A metabolite derived from metabolic $N$ hydroxylation was not detected.

M11 m/z 394.068 corresponding to a combination of both metabolic hydroxylation and desaturation was not further identified. However, based on chromatographic retention time matching it was concluded that M11 is neither a beta-keto (entry 32) or nitrone (entry 33) metabolite. 


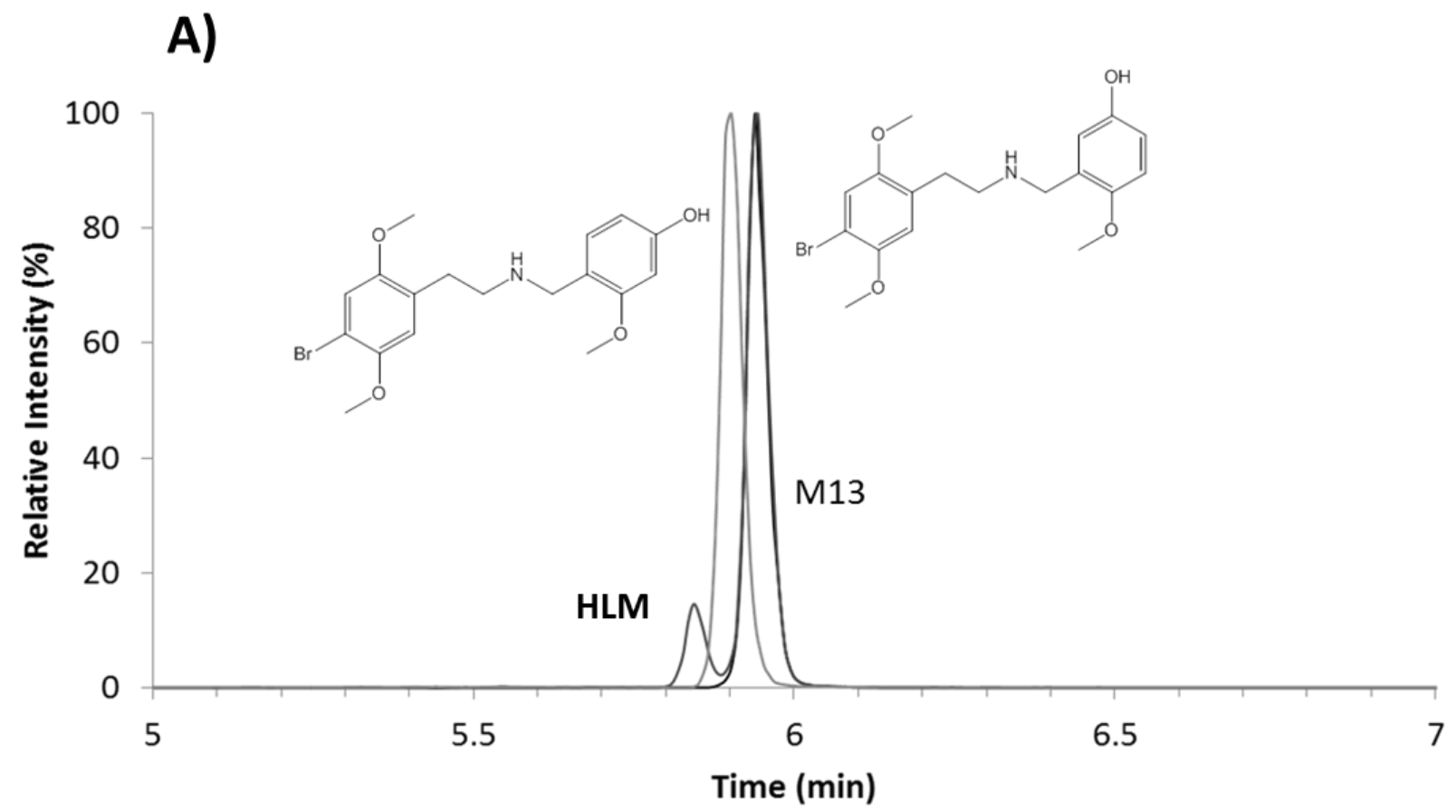

B)

M15

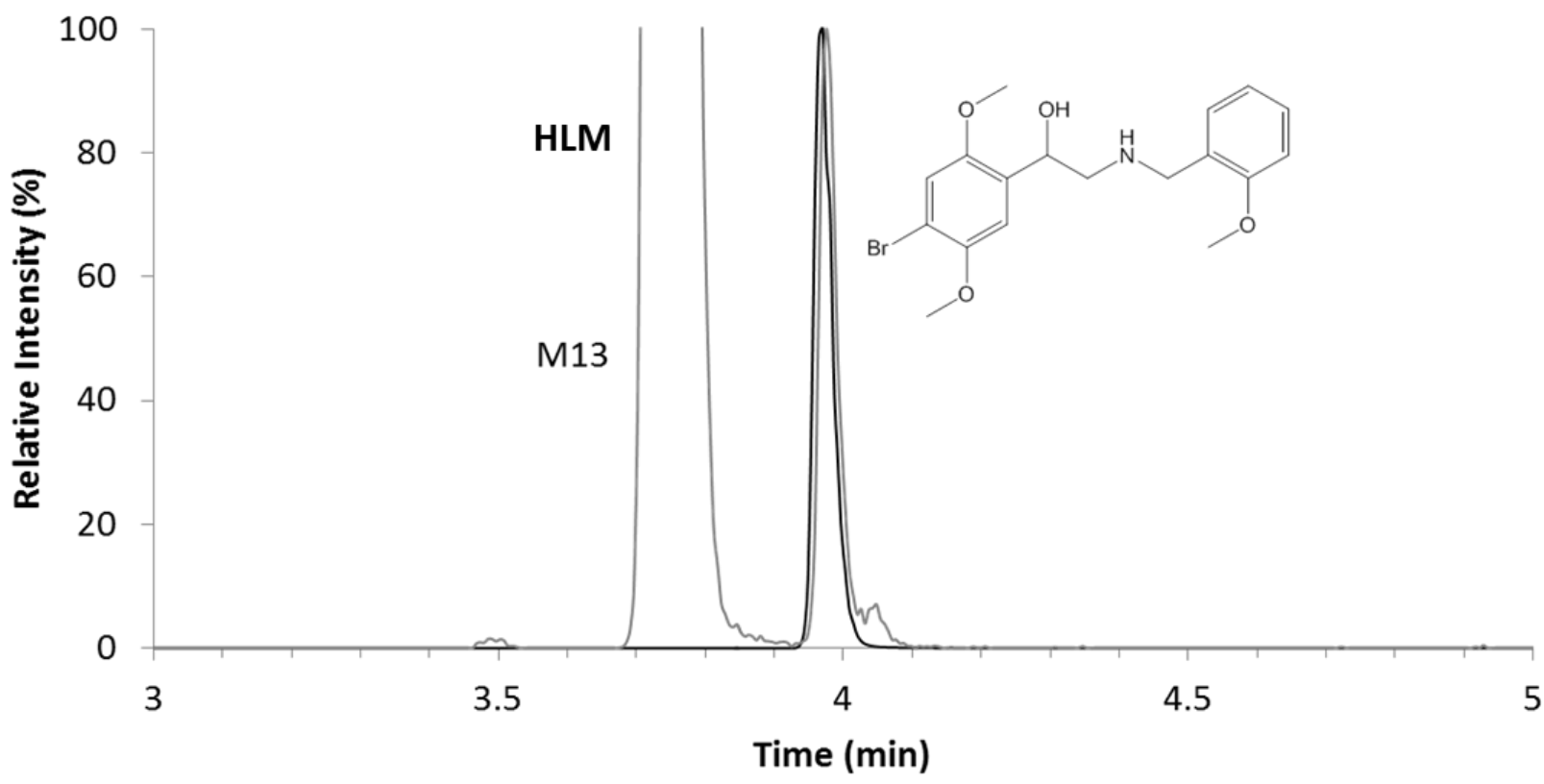

Figure S6. 


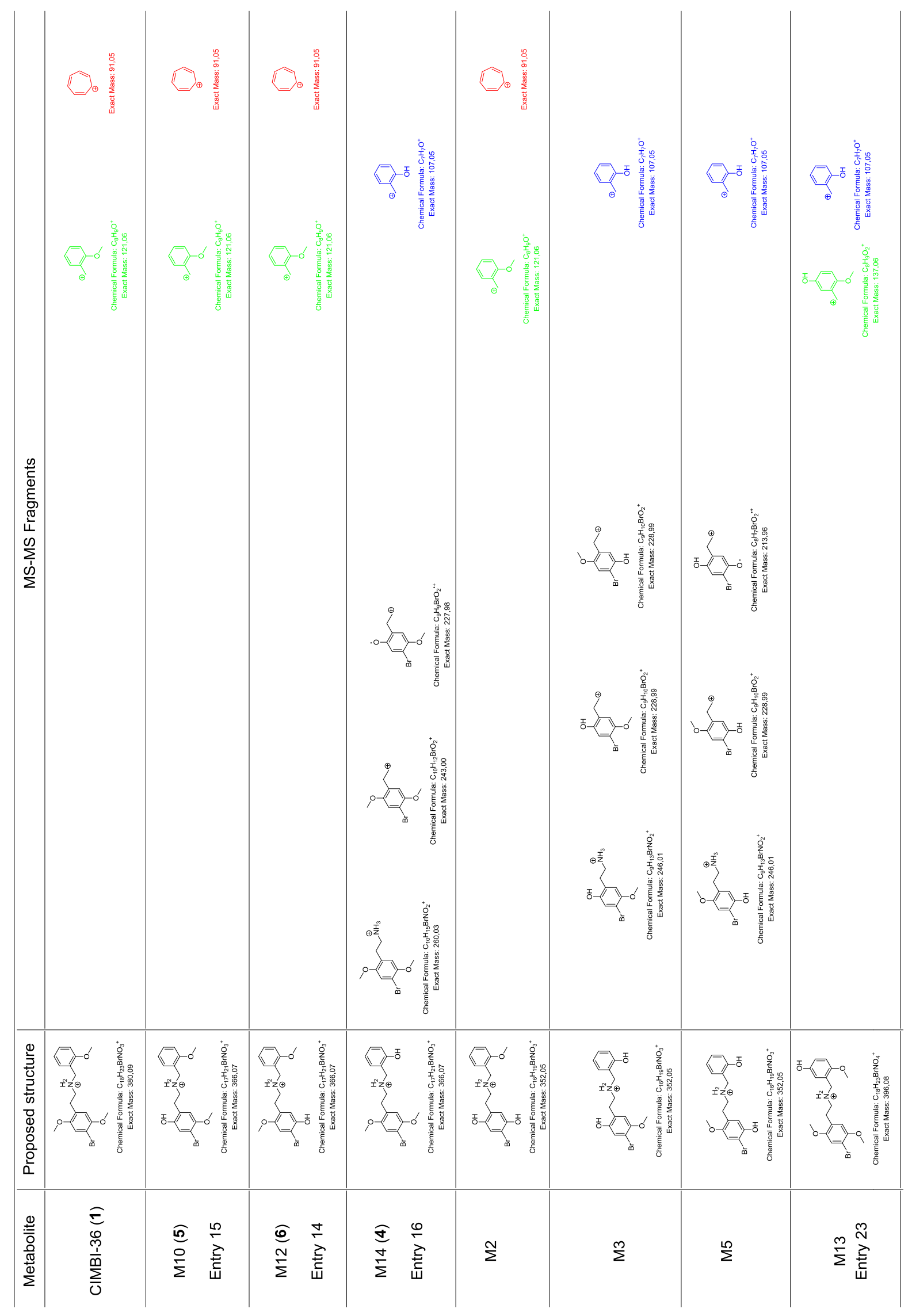




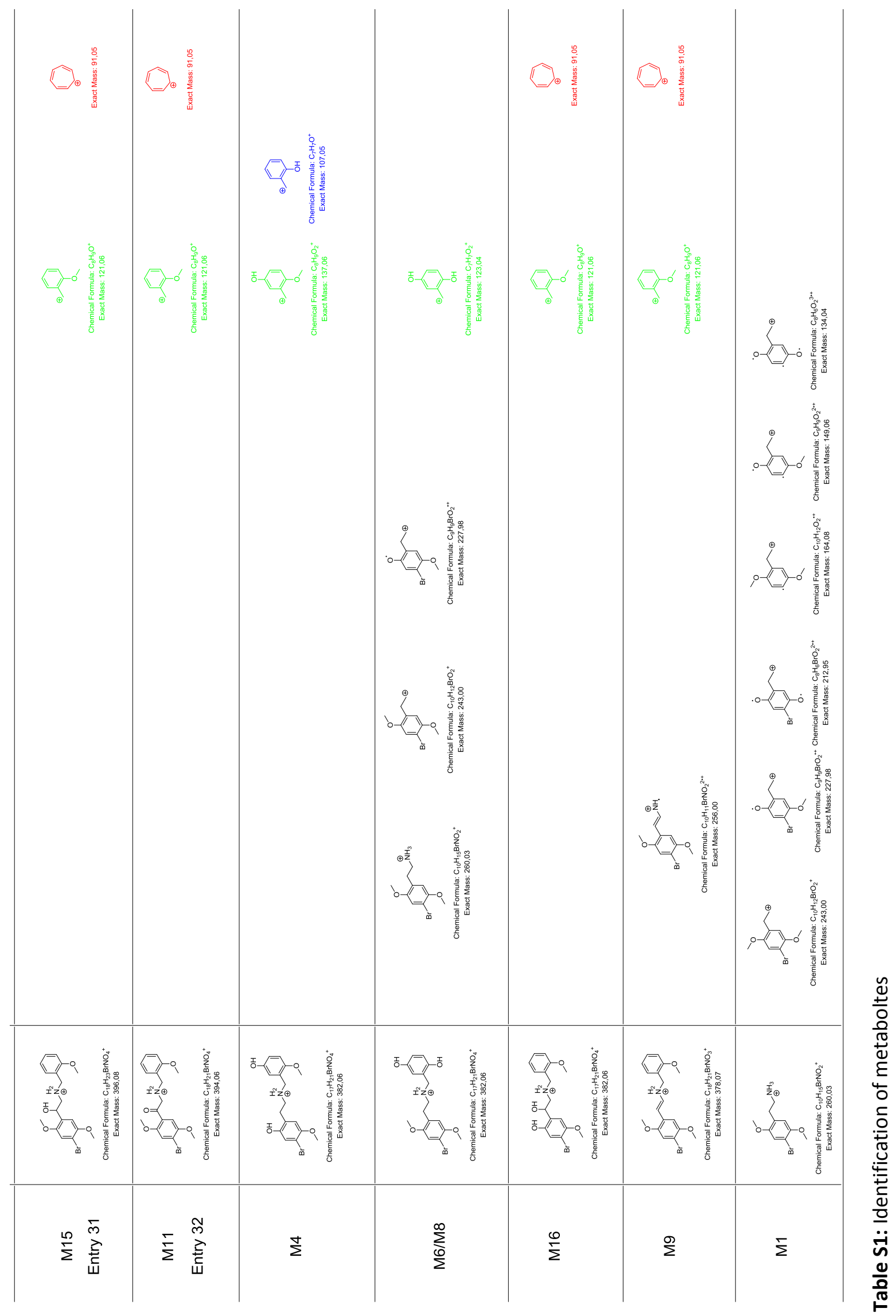




\section{Organic synthesis}

General procedures

All reagents and solvents were purchased from Aldrich and used without further purification. Thin layer chromatography was carried out on Merck silica gel $60 \mathrm{~F}_{254}$ plates, and detection took place using UV (254 and $366 \mathrm{~nm})$. Merck silica gel $(0.040-0.063 \mathrm{~mm})$ was used for flash column chromatography, and Merck silica gel $(0.015-0.040 \mathrm{~mm})$ was used for dry column vacuum chromatography. ${ }^{1}{ }^{1} \mathrm{H}$ and ${ }^{13} \mathrm{C}$ NMR was recorded on a Bruker Avance $400 \mathrm{MHz}$ spectrometer equipped with a $5 \mathrm{~mm}$ PABBO $\mathrm{BB}\left\{{ }^{1} \mathrm{H},{ }^{19} \mathrm{~F}\right\}$ Z-GRD probe, at $300 \mathrm{~K}$, a $600 \mathrm{MHz}$ Bruker Avance III HD ultra-shielded standard-bore magnet equipped with a 5$\mathrm{mm}$ cryogenically cooled ${ }^{13} \mathrm{C} /{ }^{1} \mathrm{H}$ DCH probe head, or a Bruker 600-Avance-III spectrometer equipped with a $5 \mathrm{~mm} \mathrm{TCl}$ cryoprobe operating at $600.16 \mathrm{MHz}$ for ${ }^{1} \mathrm{H}-\mathrm{NMR}$ and $150.91 \mathrm{MHz}$ for ${ }^{13} \mathrm{C}-\mathrm{NMR}$. TMS was used as internal reference for ${ }^{1} \mathrm{H}$ and the solvent non-deterrent $\left(\mathrm{CDCl}_{3}=7.26 \mathrm{ppm}\right.$ for ${ }^{1} \mathrm{H}$ and 49.00 for ${ }^{13} \mathrm{C} ; \mathrm{d}_{6}$-DMSO $=2.50 \mathrm{ppm}$ for ${ }^{1} \mathrm{H}$ and 39.52 for ${ }^{13} \mathrm{C}$ ) was used as internal reference for ${ }^{13} \mathrm{C}$ NMR. Data are tabulated in the following order: chemical shift $(\delta)$ [multiplicity (b, broad; $s$, singlet; $d$, doublet; $t$, triplet; $q$, quartet; sept, septet; $m$, multiplet), coupling constant(s) $J(\mathrm{~Hz})$, number of protons]. LC/MS were obtained using a Waters Acquity UPLC-MS instrument fitted with an Acquity 2.1x50mm UPLC BEH C18 $1.7 \mu \mathrm{m}$ column, and an APPI positive ion source. The column was heated at $60^{\circ} \mathrm{C}$ and the flow was $1.2 \mathrm{~mL} / \mathrm{min}$. PDA: $254 \mathrm{~nm}$, probe temperature: $450{ }^{\circ} \mathrm{C}$, source temperature: $150{ }^{\circ} \mathrm{C}$. Solvent system: $\mathrm{A}=\mathrm{H}_{2} \mathrm{O}: \mathrm{TFA}$ (100:0.05) and $\mathrm{B}=\mathrm{CH}_{3} \mathrm{CN}: \mathrm{TFA}(100: 0.035)$, linear gradient elution with $10 \% \mathrm{~B}$ to $100 \% \mathrm{~B}$ in 1 minute then $10 \% \mathrm{~B}$ in $0.2 \mathrm{~min}$.

Entry 1: 4-isopropoxyphenol<smiles>CC(C)Oc1ccc(O)cc1</smiles>

Hydroquinone (20.00 g, $181.63 \mathrm{mmol}, 1.00 \mathrm{eq}$.$) and isopropyliodide (30.88 g, 181.63 \mathrm{mmol}, 1.00 \mathrm{eq}$.$) was$ stirred in EtOH $(25 \mathrm{~mL})$ under $\mathrm{N}_{2}$ at reflux. $\mathrm{KOH}(10.72 \mathrm{~g}, 191.00 \mathrm{mmol}, 1.05$ eq.) in water $(30 \mathrm{~mL})$ was added to the reaction dropwise over 1 hour. The reaction was then kept at reflux for an additional 3 hours, cooled to ambient temperature and poured into aq. $1 \mathrm{~N} \mathrm{NaOH}$. The mixture was washed with diethyl ether, acidified with $10 \%$ aq. $\mathrm{HCl}$ and extracted twice with diethyl ether. The extract was dried over $\mathrm{Na}_{2} \mathrm{SO}_{4}$, 
filtered and concentrated by rotary evaporation. The residue was purified by dry column vacuum chromatography ( 0 to $45 \%$ EtOAc in in heptane, $\mathrm{Rf}=0.6$ in 1:1-EtOAc:heptane) yielding the product as brown oil (11.88 g, $43 \%)$.

${ }^{1} \mathrm{H}$ NMR $(600 \mathrm{MHz}$, Chloroform-d) $\delta 6.75-6.64(\mathrm{~m}, 4 \mathrm{H}), 4.75(\mathrm{~s}, 1 \mathrm{H}), 4.32($ hept, $J=6.1 \mathrm{~Hz}, 1 \mathrm{H}), 1.23(\mathrm{~d}, J=$ $6.1 \mathrm{~Hz}, 6 \mathrm{H}) .{ }^{13} \mathrm{C}$ NMR (151 MHz, Chloroform-d) $\delta 151.81,149.66,117.82,116.05,71.30,22.12$.

Entry 2: 2-hydroxy-5-isopropoxybenzaldehyde<smiles>CC(C)Oc1ccc(O)c(C=O)c1</smiles>

4-isopropoxyphenol (11.88 g, $78.06 \mathrm{mmol}, 1.00$ eq.) was dissolved in $20 \mathrm{~mL}$ dry $\mathrm{MeOH}, \mathrm{Mg}(1.14 \mathrm{~g}, 46.87$ mmol, 0.60 eq.) was added, and the mixture was brought to reflux under $\mathrm{N}_{2}$. More several portions of more $\mathrm{MeOH}$ was added to keep the mixture stirring. When all the $\mathrm{Mg}$ had reacted toluene $(100 \mathrm{~mL})$ was added and the $\mathrm{MeOH}$ distilled off (using a Dean Stark trap) until the reaction temperature reached $95{ }^{\circ} \mathrm{C}$. Then formaldehyde ( $8.44 \mathrm{~g}, 281.01 \mathrm{mmol}, 3.60$ eq.) was added as a slurry in toluene $(50 \mathrm{~mL})$ in 4 portions over one hour. The reaction was then kept at $95{ }^{\circ} \mathrm{C}$ for one more hour, then cooled to $20-30{ }^{\circ} \mathrm{C}$ and poured onto $10 \%$ aq. $\mathrm{HCl}(400 \mathrm{~mL})$. The mixture was stirred overnight, separated and the aq. phase extracted twice with EtOAc. The combined organic phases were washed with brine, dried over $\mathrm{MgSO}_{4}$, filtered and concentrated by rotary evaporation. The residue was purified by dry column vacuum chromatography ( 0 to $45 \%$ EtOAc in heptane, $\mathrm{Rf}=0.5$ in 1:1-EtOAc:heptane) yielding the product as a golden-brown crystalline solid (5.594 $\mathrm{g}$, $40 \%)$.

${ }^{1} \mathrm{H}$ NMR (600 MHz, Chloroform-d) $\delta 10.56(\mathrm{~s}, 1 \mathrm{H}), 9.76(\mathrm{~s}, 1 \mathrm{H}), 7.06$ (dd, $\left.J=9.0,3.0 \mathrm{~Hz}, 1 \mathrm{H}\right), 6.95$ (d, $J=3.0$ $\mathrm{Hz}, 1 \mathrm{H}), 6.85(\mathrm{~d}, J=9.0 \mathrm{~Hz}, 1 \mathrm{H}), 4.38$ (hept, $J=6.0 \mathrm{~Hz}, 1 \mathrm{H}), 1.26(\mathrm{~d}, J=6.0 \mathrm{~Hz}, 6 \mathrm{H}) .{ }^{13} \mathrm{C} \mathrm{NMR}(151 \mathrm{MHz}$, Chloroform-d) $\delta 196.15,156.05,150.83,127.39,120.27,118.89,118.61,71.47,22.00$. 
Entry 3: 2-hydroxy-5-methoxybenzaldehyde<smiles>COc1ccc(O)c(C=O)c1</smiles>

The compound was made following the same procedure as for 2 except using and 2-methoxyphenol as the starting material.

${ }^{1} \mathrm{H}$ NMR (600 MHz, Chloroform-d) $\delta 10.57(\mathrm{~s}, 1 \mathrm{H}), 9.79(\mathrm{~s}, 1 \mathrm{H}), 7.08$ (dd, J = 9.1, $\left.3.1 \mathrm{~Hz}, 1 \mathrm{H}\right), 6.93$ (d, J = 3.1 $\mathrm{Hz}, 1 \mathrm{H}), 6.86(\mathrm{~d}, J=9.0 \mathrm{~Hz}, 1 \mathrm{H}), 3.75(\mathrm{~s}, 3 \mathrm{H}) .{ }^{13} \mathrm{C}$ NMR (151 MHz, Chloroform-d) $\delta$ 196.12, 156.08, 152.74, $125.26,120.05,118.73,115.21,55.94$.

Entry 4: 5-isopropoxy-2-methoxybenzaldehyde<smiles>COc1ccc(OC(C)C)cc1C=O</smiles>

2-hydroxy-5-isopropoxybenzaldehyde (10.00 g, $55.49 \mathrm{mmol}, 1.00$ eq.), Mel (5.00 mL, $80.32 \mathrm{mmol}, 1.45$ eq.) and $\mathrm{K}_{2} \mathrm{CO}_{3}(10.00 \mathrm{~g}, 72.36 \mathrm{mmol}, 1.30$ eq.) was added to DMSO (200 mL) and stirred at ambient temperature overnight. The mixture was partitioned between diethyl ether and brine, the aq. phase separated and extracted twice with diethyl ether. The combined ether extracts were washed with water and brine, dried over $\mathrm{MgSO}_{4}$, filtered and concentrated. The residue was purified by a plug of silica (eluting with $50 \%$ EtOAc in heptane), yielding the product as an off-white solid (10.00 g, $93 \%)$.

${ }^{1} \mathrm{H}$ NMR (600 MHz, Chloroform-d) $\delta 10.36(\mathrm{~s}, 1 \mathrm{H}), 7.27(\mathrm{~d}, J=3.2 \mathrm{~Hz}, 1 \mathrm{H}), 7.04(\mathrm{dd}, J=9.0,3.2 \mathrm{~Hz}, 1 \mathrm{H}), 6.86$ $(\mathrm{d}, J=9.0 \mathrm{~Hz}, 1 \mathrm{H}), 4.43$ (hept, $J=6.1 \mathrm{~Hz}, 1 \mathrm{H}), 3.82(\mathrm{~s}, 3 \mathrm{H}), 1.24(\mathrm{~d}, J=6.1 \mathrm{~Hz}, 7 \mathrm{H}) .{ }^{13} \mathrm{C} \mathrm{NMR}(151 \mathrm{MHz}$, Chloroform-d) $\delta 189.62,156.61,151.80,125.50,125.06,113.37,113.28,70.91,56.13,21.98$. 
Entry 5: 2-isopropoxy-5-methoxybenzaldehyde<smiles>COc1ccc(OC(C)C)c(C=O)c1</smiles>

The compound was made following the same procedure as for entry 4 except using isopropyl iodide and 2hydroxy-5-methoxybenzaldehyde as the starting materials, and heating the reaction to $60{ }^{\circ} \mathrm{C}$ overnight.

${ }^{1} \mathrm{H}$ NMR (600 MHz, Chloroform-d) $\delta 10.38(s, 1 \mathrm{H}), 7.24(\mathrm{~d}, J=3.3 \mathrm{~Hz}, 1 \mathrm{H}), 7.04(\mathrm{dd}, J=9.1,3.3 \mathrm{~Hz}, 1 \mathrm{H}), 6.89$ $(\mathrm{d}, J=9.1 \mathrm{~Hz}, 1 \mathrm{H}$ ), 4.50 (hept, $J=6.0 \mathrm{~Hz}, 1 \mathrm{H}), 3.73(\mathrm{~s}, 3 \mathrm{H}), 1.30(\mathrm{~d}, J=6.0 \mathrm{~Hz}, 6 \mathrm{H}) .{ }^{13} \mathrm{C} \mathrm{NMR}(151 \mathrm{MHz}$, Chloroform-d) $\delta$ 190.01, 155.39, 153.64, 126.40, 123.73, 116.81, 109.78, 72.28, 55.78, 22.07.

Entry 6: 5-isopropoxy-2-methoxy-beta-nitrostyrene<smiles>COc1ccc(OC(C)C)cc1/C=C/[N+](=O)[O-]</smiles>

5-isopropoxy-2-methoxybenzaldehyde (4.56 g, $23.47 \mathrm{mmol}, 1.00$ eq.), MeNO2 (1.50 mL, $28.16 \mathrm{mmol}, 1.20$ eq.) and propylenediammonium diacetate $(0.456 \mathrm{~g}, 2.35 \mathrm{mmol}, 0.10$ eq.) was dissolved in iPA (10 mL) and stirred at reflux for 18 hours under $\mathrm{N}_{2}$. The mixture was partitioned between EtOAc and brine, the aq. phase separated and extracted twice with EtOAc. The combined organic phases were dried over $\mathrm{MgSO}_{4}$, filtered and concentrated by rotary evaporation. The residue was purified by flashchromatography (10 \% EtOAc in heptane $\mathrm{Rf}=0.5$ in 1:1-EtOAc:heptane), yielding the product as a light yellow crystalline solid (3.146 g, $56 \%)$.

${ }^{1} \mathrm{H}$ NMR $(400 \mathrm{MHz}$, Chloroform-d) $\delta 8.03(\mathrm{~d}, J=13.6 \mathrm{~Hz}, 1 \mathrm{H}), 7.77(\mathrm{~d}, J=13.6 \mathrm{~Hz}, 1 \mathrm{H}), 6.96-6.89(\mathrm{~m}, 2 \mathrm{H})$, $6.82(\mathrm{~d}, J=8.9 \mathrm{~Hz}, 1 \mathrm{H}), 4.43-4.32(\mathrm{~m}, 1 \mathrm{H}), 3.83(\mathrm{~s}, 3 \mathrm{H}), 1.25(\mathrm{~d}, J=6.0 \mathrm{~Hz}, 6 \mathrm{H}) .{ }^{13} \mathrm{C} N M R(101 \mathrm{MHz}$, Chloroform-d) $\delta 153.98,151.72,138.40,135.30,121.62,119.59,119.23,112.38,71.24,55.97,22.01$. 
Entry 7: 2-isopropoxy-5-methoxy-beta-nitrostyrene<smiles>COc1ccc(OC(C)C)c(/C=C/[N+](=O)[O-])c1</smiles>

The compound was made following the same procedure as for entry 6 except using 2-isopropoxy-5methoxybenzaldehyde as the starting material.

${ }^{1} \mathrm{H}$ NMR $(400 \mathrm{MHz}$, Chloroform-d) $\delta 8.06(\mathrm{~d}, J=13.6 \mathrm{~Hz}, 1 \mathrm{H}), 7.78(\mathrm{~d}, J=13.6 \mathrm{~Hz}, 1 \mathrm{H}), 6.97-6.80(\mathrm{~m}, 3 \mathrm{H})$, 4.53 (hept, $J=6.0 \mathrm{~Hz}, 1 \mathrm{H}$ ), $3.73(\mathrm{~s}, 3 \mathrm{H}), 1.33(\mathrm{~d}, J=6.0 \mathrm{~Hz}, 6 \mathrm{H}) .{ }^{13} \mathrm{C}$ NMR $(101 \mathrm{MHz}$, Chloroform-d) $\delta 153.38$, $52.40,138.25,135.56,120.53,119.49,115.73,115.20,71.84,55.84,22.11$.

Entry 8: 5-isopropoxy-2-methoxyphenethylamine hydrochloride<smiles>COc1ccc(OC(C)C)cc1CCN</smiles>

5-isopropoxy-2-methoxy-beta-nitrostyrene $(6.349 \mathrm{~g}, 26.76 \mathrm{mmol}, 1.00$ eq.) dissolved in dry THF (100 $\mathrm{mL})$ was added (dropwise via addition funnel) to a refluxing suspension of $\mathrm{LiAlH}_{4}(3.12 \mathrm{~g}, 82.21 \mathrm{mmol}, 3.07$ eq.) in dry THF $(50 \mathrm{~mL})$ under $\mathrm{N}_{2}$. After complete addition, the reaction was kept at reflux for 2 hours, before being cooled with an ice bath and quenched (successive careful addition of water ( $3 \mathrm{~mL}$ ), $16 \%$ aq. $\mathrm{NaOH}$ (3 $\mathrm{mL})$, water $(9 \mathrm{~mL}), \mathrm{Na}_{2} \mathrm{SO}_{4}(50 \mathrm{~g})$, and diethyl ether $\left.(300 \mathrm{~mL})\right)$. The resulting white solids were removed by filtration and the filtercake washed with diethyl ether. The solvent was removed by rotary evaporation. The residue was dissolved in dry diethyl ether, and the product was crystallized by adding $4 \mathrm{n} \mathrm{HCl}$ in dioxane, collecting by filtration and drying the white crystals $(4.431 \mathrm{~g}, 67 \%)$ under high vaccum.

${ }^{1} \mathrm{H}$ NMR $\left(600 \mathrm{MHz}\right.$, DMSO- $\left.d_{6}\right) \delta 8.16(\mathrm{brs}, 3 \mathrm{H}), 6.89(\mathrm{~d}, J=8.6 \mathrm{~Hz}, 1 \mathrm{H}), 6.83-6.75(\mathrm{~m}, 2 \mathrm{H}), 4.47$ (hept, $J=6.0$ $\mathrm{Hz}, 1 \mathrm{H}$ ), $3.74(\mathrm{~s}, 3 \mathrm{H}), 2.95$ (dd, $J=9.1,6.6 \mathrm{~Hz}, 2 \mathrm{H}), 2.84(\mathrm{dd}, J=9.1,6.6 \mathrm{~Hz}, 2 \mathrm{H}), 1.23(\mathrm{~d}, J=6.0 \mathrm{~Hz}, 6 \mathrm{H}) .{ }^{13} \mathrm{C}$ NMR $\left(151 \mathrm{MHz}, \mathrm{DMSO}-d_{6}\right) \delta 151.69,151.58,126.67,118.94,115.07,112.24,70.17,56.22,38.95,28.46$, 22.40 . 
Entry 9: 2-isopropoxy-5-methoxyphenethylamine hydrochloride<smiles>COc1ccc(OC(C)C)c(CCN)c1</smiles>

The compound was made following the same procedure as for entry 8 except using 2-isopropoxy-5methoxy-beta-nitrostyrene as the starting material.

${ }^{1} \mathrm{H}$ NMR (600 MHz, DMSO- $\left.d_{6}\right) \delta 8.11(\mathrm{brs}, 3 \mathrm{H}), 6.96-6.91(\mathrm{~m}, 1 \mathrm{H}), 6.82-6.75(\mathrm{~m}, 2 \mathrm{H}), 4.49$ (hept, $J=6.0$ $\mathrm{Hz}, 1 \mathrm{H}), 3.70(\mathrm{~s}, 3 \mathrm{H}), 2.99-2.93(\mathrm{~m}, 2 \mathrm{H}), 2.84(\mathrm{dd}, J=9.3,6.5 \mathrm{~Hz}, 2 \mathrm{H}), 1.25(\mathrm{~d}, J=6.0 \mathrm{~Hz}, 6 \mathrm{H}) .{ }^{13} \mathrm{C}$ NMR $(151$ MHz, DMSO- $\left.d_{6}\right) \delta 153.50,149.75,128.06,116.58,115.54,112.96,70.80,55.75,39.01,28.72,22.45$.

Entry 10: 4-bromo-5-isopropoxy-2-methoxyphenethylamine hydrochloride<smiles>COc1cc(Br)c(OC(C)C)cc1CCN</smiles>

5-isopropoxy-2-methoxyphenethylamine ( $7.357 \mathrm{~g}, 35.15 \mathrm{mmol}, 1.00$ eq.) was dissolved in $30 \mathrm{~mL} \mathrm{AcOH}$ and stirred under $\mathrm{N}_{2}$. Elemental bromine $(1.81 \mathrm{~mL}, 35.15 \mathrm{mmol}, 1.00 \mathrm{eq})$ in $\mathrm{AcOH}(10 \mathrm{~mL})$ was added dropwise, and the reaction stirred for 2 hours at ambient temperature. The mixture was poured into water, $\mathrm{Na}_{2} \mathrm{SO}_{3}$ was added, followed by $35 \%$ aq. $\mathrm{NaOH}$ until $\mathrm{pH}>10$. The mixture was extracted with diethyl ether three times and the combined extracts dried over $\mathrm{MgSO}_{4}$, filtered and concentrated by rotary evaporation. The residue was purified by flashchromatography (85:10:5-EtOAc:MeOH:triethylamine, $\mathrm{Rf}=0.2)$, and the product precipitated as a white crystalline solid $(6.657 \mathrm{~g}, 58 \%)$ by dissolving the freebase in diethyl ether, adding $4 \mathrm{~N} \mathrm{HCl}$ in dioxane.

${ }^{1} \mathrm{H}$ NMR $(600 \mathrm{MHz}$, Chloroform-d) $\delta 8.33$ (brs, 2H), 7.05 (s, 1H), $6.92(\mathrm{~s}, 1 \mathrm{H}), 4.44$ (hept, $J=6.1 \mathrm{~Hz}, 1 \mathrm{H}), 3.82$ (s, 3H), $3.28-3.20(\mathrm{~m}, 2 \mathrm{H}), 3.03(\mathrm{t}, J=7.6 \mathrm{~Hz}, 2 \mathrm{H}), 1.34(\mathrm{~d}, J=6.1 \mathrm{~Hz}, 6 \mathrm{H}) .{ }^{13} \mathrm{C}$ NMR $(151 \mathrm{MHz}$, Chloroformd) $\delta 152.51,148.49,124.36,120.87,115.74,113.66,74.00,55.98,39.16,29.48,22.22$. 
Entry 11: 4-bromo-2-isopropoxy-5-methoxyphenethylamine hydrochloride<smiles>COc1cc(CCN)c(OC(C)C)cc1Br</smiles>

The compound was made following the same procedure as for entry 10 except using 2-isopropoxy-5methoxyphenethylamine as the starting material.

${ }^{1} \mathrm{H}$ NMR (400 MHz, Chloroform-d) $\delta 8.19$ (brs, 2H), $6.98(\mathrm{~s}, 1 \mathrm{H}), 6.81(\mathrm{~s}, 1 \mathrm{H}), 4.38(\mathrm{~h}, J=6.1 \mathrm{~Hz}, 1 \mathrm{H}), 3.77(\mathrm{~s}$, $3 \mathrm{H}), 3.15(\mathrm{t}, J=7.4 \mathrm{~Hz}, 2 \mathrm{H}), 2.95(\mathrm{dd}, J=8.5,6.3 \mathrm{~Hz}, 2 \mathrm{H}), 1.25$ (d, $J=6.1 \mathrm{~Hz}, 6 \mathrm{H}) .{ }^{13} \mathrm{C}$ NMR $(101 \mathrm{MHz}$, Chloroform-d) $\delta 150.13,149.93,125.71,118.78,115.50,110.46,71.45,57.12,39.34,29.50,22.10$.

Entry 12: N-(2-methoxybenzyl)-4-bromo-5-isoproxy-2-methoxyphenethylamine hydrochloride<smiles>COc1cc(Br)c(OC(C)C)cc1CCNCc1ccccc1OC</smiles>

4-bromo-5-isopropoxy-2-methoxyphenethylamine hydrochloride $(1.00 \mathrm{~g}, 3,08 \mathrm{mmol}, 1.00$ eq.) 2methoxybenzaldehyde $(0.50 \mathrm{~g}, 3.67 \mathrm{mmol}, 1.19$ eq.) and triethylamine ( $1.50 \mathrm{~mL}, 10.75 \mathrm{mmol}, 3.49$ eq.) was dissolved in dry dichloromethane ( $30 \mathrm{~mL}$ ). $3 \AA$ A molecular sieves (a tablespoon) were added and the reaction stirred for 30 minutes under argon. Sodium triacetoxyborohydride $(2.61 \mathrm{~g}, 12.32 \mathrm{mmol}, 4.00 \mathrm{eq}$.) was then added, and the reaction stirred overnight. The mixture was then filtered through celite, concentrated by rotary evaporation, partitioned between EtOAc and brine, and the aq. Phase separated and extracted twice with EtOAc. The combined organic phases were dried $\left(\mathrm{Na}_{2} \mathrm{SO}_{4}\right)$, filtered and concentrated. The residue was purified by flashchromatography (45:50:5-Heptane:EtOAc:Triethylamine, $\mathrm{Rf}=0.2)$. The product was crystallized by dissolving the freebase in diethyl ether $(10 \mathrm{~mL})$ and adding $1 \mathrm{~mL} 4 \mathrm{M} \mathrm{HCl}$ in dioxane, followed by heptane until the solution became cloudy $(30-40 \mathrm{~mL})$, then placing the vessel at 2-5 C overnight. The resulting white crystalline solid $(0.286 \mathrm{~g}, 21 \%)$ was collected by filtration and dried at high vacuum.

${ }^{1} \mathrm{H}$ NMR $(600 \mathrm{MHz}$, Chloroform-d) $\delta 9.34$ (brs, 2H), 7.29 (dd, J = 7.5, 1.7 Hz, 1H), 7.27 - 7.22 (m, 1H), $6.90-$ $6.84(\mathrm{~m}, 2 \mathrm{H}), 6.80-6.73(\mathrm{~m}, 2 \mathrm{H}), 4.35(\mathrm{p}, J=6.1 \mathrm{~Hz}, 1 \mathrm{H}), 4.06(\mathrm{~s}, 2 \mathrm{H}), 3.66(\mathrm{~s}, 3 \mathrm{H}), 3.54(\mathrm{~s}, 3 \mathrm{H}), 3.00$ (brs, $4 \mathrm{H}), 1.25(\mathrm{~d}, J=6.1 \mathrm{~Hz}, 6 \mathrm{H}) .{ }^{13} \mathrm{C}$ NMR (151 MHz, Chloroform-d) $\delta 157.59,152.03,148.69,132.02,131.17$, 
Entry 13: N-(2-methoxybenzyl)-4-bromo-2-isoproxy-5-methoxyphenethylamine hydrochloride<smiles>COc1cc(CCNCc2ccccc2OC)c(OC(C)C)cc1Br</smiles>

The compound was synthesized using the same procedure as for entry 12 except using 4-bromo-2isopropoxy-5-methoxyphenethylamine hydrochloride as the starting material.

${ }^{1} \mathrm{H}$ NMR $\left(600 \mathrm{MHz}, \mathrm{DMSO}-d_{6}\right) \delta 9.08(\mathrm{brs}, 2 \mathrm{H}), 7.48(\mathrm{dd}, J=7.6,1.7 \mathrm{~Hz}, 1 \mathrm{H}), 7.45-7.40(\mathrm{~m}, 1 \mathrm{H}), 7.23(\mathrm{~s}, 1 \mathrm{H})$, $7.10(\mathrm{~d}, J=8.3 \mathrm{~Hz}, 1 \mathrm{H}), 7.04-6.98(\mathrm{~m}, 2 \mathrm{H}), 4.54(\mathrm{p}, J=6.0 \mathrm{~Hz}, 1 \mathrm{H}), 4.18-4.11(\mathrm{~m}, 2 \mathrm{H}), 3.84(\mathrm{~s}, 3 \mathrm{H}), 3.80(\mathrm{~s}$, $3 \mathrm{H}), 3.12-3.03(\mathrm{~m}, 2 \mathrm{H}), 2.99-2.91(\mathrm{~m}, 2 \mathrm{H}), 1.20(\mathrm{~d}, J=6.0 \mathrm{~Hz}, 6 \mathrm{H}) .{ }^{13} \mathrm{C}$ NMR $\left(151 \mathrm{MHz}, \mathrm{DMSO}-d_{6}\right) \delta$ $157.96,150.00,131.88,131.30,127.29,120.88,120.11,119.20,115.35,111.60,109.44,71.28,57.03$, $56.09,46.21,45.36,27.10,22.20,8.94$.

Entry 14: N-(2-methoxybenzyl)-4-bromo-5-hydroxy-2-methoxyphenethylamine hydrochloride<smiles>COc1cc(Br)c(O)cc1CCNCc1ccccc1OC</smiles>

$\mathrm{N}$-(2-methoxybenzyl)-4-bromo-5-isoproxy-2-methoxyphenethylamine hydrochloride $(0.50 \mathrm{~g}, 1.12 \mathrm{mmol}$, 1.00 eq.) was dissolved in dry dichloromethane $(20 \mathrm{~mL}$ ), stirred under argon, and cooled to $-78 \mathrm{C}$ (dry ice and acetone bath). $1 \mathrm{M} \mathrm{BCl}_{3}$ in dichloromethane (1.68 mL, $1.68 \mathrm{mmol}, 1.50$ eq.) was added dropwise. The reaction was stirred overnight and slowly allowed to reach ambient temperature, before being quenched by careful addition of $10 \mathrm{~mL} 2 \mathrm{M} \mathrm{HCl}$ in $\mathrm{MeOH}$. After stirring for 5 minutes, the mixture was concentrated by rotary evaporation, chased several times with $\mathrm{MeOH}$ (to azeotropically remove trimethyl borate). The residue was then diluted with diethyl ether causing the product to precipitate as a white crystalline solid (0.305 g, $67 \%)$.

${ }^{1} \mathrm{H}$ NMR $\left(600 \mathrm{MHz}, \mathrm{DMSO}-d_{6}\right) \delta 9.78(\mathrm{~s}, 1 \mathrm{H}), 8.93(\mathrm{brs}, 2 \mathrm{H}), 7.48-7.40(\mathrm{~m}, 2 \mathrm{H}), 7.11(\mathrm{dd}, J=8.3,1.0 \mathrm{~Hz}, 1 \mathrm{H})$, $7.09(\mathrm{~s}, 1 \mathrm{H}), 7.02(\mathrm{td}, J=7.4,1.0 \mathrm{~Hz}, 1 \mathrm{H}), 6.81(\mathrm{~s}, 1 \mathrm{H}), 4.14(\mathrm{~s}, 2 \mathrm{H}), 3.85(\mathrm{~s}, 3 \mathrm{H}), 3.72(\mathrm{~s}, 3 \mathrm{H}), 3.05-2.99(\mathrm{~m}$, 
2H), $2.89-2.84(\mathrm{~m}, 2 \mathrm{H}) .{ }^{13} \mathrm{C}$ NMR (151 MHz, DMSO- $\left.d_{6}\right) \delta 157.93,150.85,148.28,131.89,131.34,125.74$, $120.87,120.10,118.35,115.88,111.58,107.73,56.57,56.09,46.44,45.44,26.82$.

Entry 15: N-(2-methoxybenzyl)-4-bromo-2-hydroxy-5-methoxyphenethylamine hydrochloride<smiles>COc1cc(CCNCc2ccccc2OC)c(O)cc1Br</smiles>

The compound was synthesized using the same procedure as for entry 14, except using N-(2methoxybenzyl)-4-bromo-2-isoproxy-5-methoxyphenethylamine hydrochloride as the starting material.

${ }^{1} \mathrm{H}$ NMR $\left(600 \mathrm{MHz}, \mathrm{DMSO}-d_{6}\right) \delta 9.74(\mathrm{~s}, 1 \mathrm{H}), 9.02(\mathrm{brs}, 2 \mathrm{H}), 7.48(\mathrm{dd}, J=7.5,1.7 \mathrm{~Hz}, 1 \mathrm{H}), 7.45-7.41(\mathrm{~m}, 1 \mathrm{H})$, $7.10(\mathrm{dd}, J=8.4,1.0 \mathrm{~Hz}, 1 \mathrm{H}), 7.08(\mathrm{~s}, 1 \mathrm{H}), 7.01(\mathrm{td}, J=7.4,1.0 \mathrm{~Hz}, 1 \mathrm{H}), 6.91(\mathrm{~s}, 1 \mathrm{H}), 4.17-4.11(\mathrm{~m}, 2 \mathrm{H}), 3.84$ $(\mathrm{s}, 3 \mathrm{H}), 3.76(\mathrm{~s}, 3 \mathrm{H}), 3.14-3.05(\mathrm{~m}, 2 \mathrm{H}), 2.96-2.89(\mathrm{~m}, 2 \mathrm{H}) .{ }^{13} \mathrm{C}$ NMR $\left(151 \mathrm{MHz}, \mathrm{DMSO}-d_{6}\right) \delta 157.95$, $150.15,148.77,131.94,131.31,124.02,120.85,120.15,119.52,115.37,111.57,109.04,57.02$, 56.07, $46.35,45.45,26.98$.

Entry 16: N-(2-hydroxybenzyl)-4-bromo-2,5-dimethoxyphenethylamine hydrochloride<smiles>COc1cc(CCNCc2ccccc2O)c(OC)cc1Br</smiles>

This compound was synthesized as previously described. ${ }^{2}$

${ }^{1} \mathrm{H}$ NMR $\left(600 \mathrm{MHz}, \mathrm{DMSO}-d_{6}\right) \delta 10.36(\mathrm{~s}, 1 \mathrm{H}), 9.21(\mathrm{~s}, 2 \mathrm{H}), 7.42(\mathrm{dd}, J=7.6,1.7 \mathrm{~Hz}, 1 \mathrm{H}), 7.25-7.21(\mathrm{~m}, 1 \mathrm{H})$, $7.19(\mathrm{~s}, 1 \mathrm{H}), 7.04-6.99(\mathrm{~m}, 2 \mathrm{H}), 6.84(\mathrm{td}, J=7.4,1.2 \mathrm{~Hz}, 1 \mathrm{H}), 4.15-4.05(\mathrm{~m}, 2 \mathrm{H}), 3.80(\mathrm{~s}, 3 \mathrm{H}), 3.75(\mathrm{~s}, 3 \mathrm{H})$, $3.10-3.01(\mathrm{~m}, 2 \mathrm{H}), 3.01-2.94(\mathrm{~m}, 2 \mathrm{H}) .{ }^{13} \mathrm{C}$ NMR $\left(151 \mathrm{MHz}, \mathrm{DMSO}-d_{6}\right) \delta 156.56,151.92,149.81,132.10$, $130.80,125.97,119.44,118.50,116.29,115.89,115.38,109.30,57.06,56.69,46.09,45.28,26.80$. 


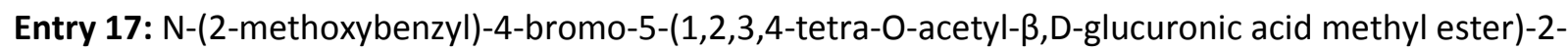
methoxyphenethylamine<smiles>COC(=O)[C@H]1O[C@@H](Oc2cc(OC)c(OC)cc2Br)[C@H](OC(C)=O)[C@@H](OC(C)=O)[C@@H]1OC(C)=O</smiles>

$\mathrm{N}$-(2-methoxybenzyl)-4-bromo-5-hydroxy-2-methoxyphenethylamine hydrochloride $(1.00 \mathrm{~g}, 2.48 \mathrm{mmol}$, 1.00 eq.) and 1,2,3,4-tetra-O-acetyl- $\beta, D$-glucuronic acid methyl ester ( $4.67 \mathrm{~g}, 12.42 \mathrm{mmol}, 5.00$ eq.) was stirred in dry dichloromethane $(30 \mathrm{~mL})$ under argon. Boron trifluoride diethyl etherate $(3.06 \mathrm{~mL}, 24.83$ mmol, 10 eq.) was added via syringe, turning the reaction mixture into a clear reddish solution. The reaction was stirred at room temperature for 2 days, during which 2 additional portions of 1,2,3,4-tetra-Oacetyl- $\beta, D$-glucuronic acid methyl ester and boron trifluoride diethyl etherate was added to the reaction. The reaction was quenched by careful addition of sat. aq. bicarbonate, separated and the aq. phase extracted 3 times with EtOAc. The combined organic phases were dried over $\mathrm{Na}_{2} \mathrm{SO}_{4}$, filtered and concentrated by rotary evaporation. The residue was purified by flash chromatography ( $100 \%$ heptane to $50 \%$ heptane with 85:10:5-EtOAc:MeOH:triethylamine, $\mathrm{Rf}=0.7$ in $5 \%$ triethylamine in EtOAc), however all fractions containing the product $\mathrm{m} / \mathrm{z}=684$ peak (LC/MS) were contaminated with tetracyl-D-Glucuronic acid methyl esther. The impure product $(3.10 \mathrm{~g}, 183 \%)$ was a white solid, and was used in the following deprotection step without further purification. 
Entry 18: N-(2-(1,2,3,4-tetra-O-acetyl- $\beta, D-$ glucuronic acid methyl ester)-benzyl)-4-bromo-2,5dimethoxyphenethylamine<smiles>COC(=O)[C@H]1O[C@@H](Oc2ccccc2CNCCc2cc(OC)c(Br)cc2OC)[C@H](OC(C)=O)[C@@H](OC(C)=O)[C@@H]1OC(C)=O</smiles>

The compound was synthesized using the same procedure as for entry 17 , except using $\mathrm{N}-(2-$ hydroxybenzyl)-4-bromo-2,5-dimethoxyphenethylamine hydrochloride as the starting material. 


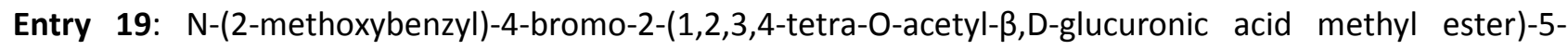
methoxyphenethylamine<smiles>COC(=O)C1OC(Oc2cc(OC)c(Br)cc2CCNCc2ccccc2OC)[C@H](O)[C@@H](OC(C)=O)[C@@H]1OC(C)=O</smiles>

The compound was synthesized using the same procedure as for entry 17 , except using $\mathrm{N}-(2-$ methoxybenzyl)-4-bromo-2-hydroxy-5-methoxyphenethylamine hydrochloride hydrochloride as the starting material.

Entry 20: glucuronide 9<smiles>COc1cc(Br)c(O[C@@H]2O[C@H](C(=O)O)[C@@H](O)[C@H](O)[C@H]2O)cc1CCNCc1ccccc1OC</smiles>

N-(2-methoxybenzyl)-4-bromo-5-(1,2,3,4-tetra-O-acetyl- $\beta, D-g l u c u r o n i c \quad$ acid methyl ester)-2methoxyphenethylamine $(1.00 \mathrm{~g}, 1.47 \mathrm{mmol})$ was dissolved in 1:9-water:MeOH $(10 \mathrm{~mL})$, followed by potassium cyanide $(0.95 \mathrm{~g}, 14.7 \mathrm{mmol}, 10 \mathrm{eq}$.). The reaction was stirred at room temperature for 30 minutes, then the reaction mixture was purified on a Waters Autopurification system with a Sunfire ${ }^{\mathrm{TM}}$ Prep $\mathrm{C} 18 \mathrm{OBD}^{\mathrm{TM}} 5 \mu \mathrm{m}, 30 \times 100 \mathrm{~mm}$ column. The column was heated at $60{ }^{\circ} \mathrm{C}$ and the flow was $90 \mathrm{~mL} / \mathrm{min}$. Fractions were collected by UV (230) and mass $(542 \mathrm{~g} / \mathrm{mol})$. Mobile phases used: $\mathrm{A}=\mathrm{H}_{2} \mathrm{O}$ containing $0.05 \%$ TFA and $\mathrm{B}=\mathrm{CH}_{3} \mathrm{CN}$ containing $0.05 \%$ TFA. The length of each run was 6 minutes and the gradient used was linear (2\% B in A to $50 \%$ B in A). Injection volume was $500 \mu \mathrm{L}$ per run. Concentrating the fractions by rotary evaporation and chasing with $\mathrm{CH}_{3} \mathrm{CN}$ to remove water yielded product as a colorless gum $(0.109 \mathrm{~g}, 14 \%)$.

${ }^{1} \mathrm{H}$ NMR $\left(600 \mathrm{MHz}\right.$, DMSO-d $\left.d_{6}\right) \delta 8.73(\mathrm{~s}, 2 \mathrm{H}), 7.49-7.39(\mathrm{~m}, 2 \mathrm{H}), 7.21(\mathrm{~s}, 1 \mathrm{H}), 7.12(\mathrm{~d}, J=8.3 \mathrm{~Hz}, 1 \mathrm{H}), 7.08(\mathrm{~s}$, $1 \mathrm{H}), 7.03(\mathrm{t}, J=7.4 \mathrm{~Hz}, 1 \mathrm{H}), 5.06(\mathrm{~d}, J=7.3 \mathrm{~Hz}, 1 \mathrm{H}), 4.16$ (brs, $2 \mathrm{H}), 3.85(\mathrm{~s}, 3 \mathrm{H}), 3.82(\mathrm{~d}, J=9.7 \mathrm{~Hz}, 1 \mathrm{H}), 3.78$ 
$(\mathrm{s}, 3 \mathrm{H}), 3.47-3.38(\mathrm{~m}, 1 \mathrm{H}), 3.34-3.27(\mathrm{~m}, 2 \mathrm{H}), 3.14-3.06(\mathrm{~m}, 2 \mathrm{H}), 2.96-2.84(\mathrm{~m}, 2 \mathrm{H}) .{ }^{13} \mathrm{C} \mathrm{NMR}(151 \mathrm{MHz}$, DMSO- $\left.d_{6}\right) \delta 170.45,157.97,153.13,147.61,131.81,131.40,125.75,120.90,120.06,119.07,116.01$, 111.59, 111.09, 101.61, 76.52, 76.06, 73.44, 71.73, 56.66, 56.06, 46.39, 45.71, 40.83, 30.88, 26.85.

Entry 21: glucuronide 8<smiles>COc1cc(CCNCc2ccccc2OC)c(OC2O[C@H](C(=O)O)[C@@H](O)[C@H](O)[C@H]2O)cc1Br</smiles>

The compound was synthesized using the same procedure as for compound entry 20 , except using $\mathrm{N}-(2-$ methoxybenzyl)-4-bromo-2-(1,2,3,4-tetra-O-acetyl- $\beta$,D-glucuronic acid methyl ester)-5methoxyphenethylamine as the starting material.

${ }^{1} \mathrm{H}$ NMR $\left(600 \mathrm{MHz}, \mathrm{DMSO}-d_{6}\right) \delta 8.72(\mathrm{~s}, 2 \mathrm{H}), 7.46-7.40(\mathrm{~m}, 2 \mathrm{H}), 7.36(\mathrm{~s}, 1 \mathrm{H}), 7.13-7.09(\mathrm{~m}, 1 \mathrm{H}), 7.05-$ $6.98(\mathrm{~m}, 2 \mathrm{H}), 4.99(\mathrm{~d}, J=7.1 \mathrm{~Hz}, 1 \mathrm{H}), 4.16(\mathrm{t}, J=5.9 \mathrm{~Hz}, 2 \mathrm{H}), 3.93(\mathrm{~d}, J=9.7 \mathrm{~Hz}, 1 \mathrm{H}), 3.86-3.79(\mathrm{~m}, 7 \mathrm{H}), 3.41$ $(\mathrm{t}, J=9.1 \mathrm{~Hz}, 1 \mathrm{H}), 3.37-3.29(\mathrm{~m}, 2 \mathrm{H}), 3.24-3.11(\mathrm{~m}, 2 \mathrm{H}), 3.04-2.92(\mathrm{~m}, 2 \mathrm{H}) .{ }^{13} \mathrm{C}$ NMR $(151 \mathrm{MHz}$, DMSO$\left.d_{6}\right) \delta 170.69,158.73,158.51,157.93,151.28,149.67,131.80,131.41,127.68,121.08,120.88,120.19$, $114.92,111.58,109.30,102.27,76.04,75.76,73.53,71.80,56.95,56.07,46.80,45.85,27.01$.

Entry 22: glucuronide 7<smiles>COc1cc(CCNCc2ccccc2O[C@@H]2O[C@H](C(=O)O)[C@@H](O)[C@H](O)[C@H]2O)c(OC)cc1Br</smiles>

The compound was synthesized using the same procedure as for compound 20 , except using $\mathrm{N}-(2-(1,2,3,4-$ tetra-O-acetyl- $\beta$,D-glucuronic acid methyl ester)-benzyl)-4-bromo-2,5-dimethoxyphenethylamine as the starting material.

${ }^{1} \mathrm{H}$ NMR $\left(600 \mathrm{MHz}\right.$, DMSO- $\left.d_{6}\right) \delta 8.90-8.78(\mathrm{~m}, 2 \mathrm{H}), 7.52-7.40(\mathrm{~m}, 2 \mathrm{H}), 7.25-7.16(\mathrm{~m}, 2 \mathrm{H}), 7.13(\mathrm{td}, J=$ 
7.5, $1.1 \mathrm{~Hz}, 1 \mathrm{H}), 6.99(\mathrm{~s}, 1 \mathrm{H}), 5.05(\mathrm{~d}, J=7.7 \mathrm{~Hz}, 1 \mathrm{H}), 4.33-4.22(\mathrm{~m}, 2 \mathrm{H}), 4.00(\mathrm{~d}, J=9.6 \mathrm{~Hz}, 1 \mathrm{H}), 3.80(\mathrm{~s}, 3 \mathrm{H})$, $3.77(\mathrm{~s}, 3 \mathrm{H}), 3.50-3.37(\mathrm{~m}, 3 \mathrm{H}), 3.19-3.11(\mathrm{~m}, 2 \mathrm{H}), 2.98-2.92(\mathrm{~m}, 2 \mathrm{H}) .{ }^{13} \mathrm{C}$ NMR $\left(151 \mathrm{MHz}, \mathrm{DMSO}-d_{6}\right) \delta$ $170.51,159.06,158.83,156.20,151.99,149.89,131.94,131.45,125.79,123.22,121.73,116.36,116.33$, $115.42,109.49,101.97,75.89,73.51,71.82,57.11,56.70,46.54,45.69,26.83$.

Entry 23: N-(5-hydroxy-2-methoxybenzyl)-4-bromo-2,5-dimethoxyphenethylamine hydrochloride<smiles>COc1cc(CCNCc2cc(O)ccc2OC)c(OC)cc1Br</smiles>

This compound was synthesized as previously described. ${ }^{2}$

${ }^{1} \mathrm{H}$ NMR $\left(600 \mathrm{MHz}\right.$, Methanol- $\left.d_{4}\right) \delta 7.20(\mathrm{~s}, 1 \mathrm{H}), 6.98-6.93(\mathrm{~m}, 2 \mathrm{H}), 6.88(\mathrm{dd}, J=8.8,2.9 \mathrm{~Hz}, 1 \mathrm{H}), 6.85(\mathrm{~d}, J=$ $2.9 \mathrm{~Hz}, 1 \mathrm{H}), 4.19(\mathrm{~s}, 2 \mathrm{H}), 3.85(\mathrm{~s}, 3 \mathrm{H}), 3.82(\mathrm{~s}, 3 \mathrm{H}), 3.81(\mathrm{~s}, 3 \mathrm{H}), 3.25-3.21(\mathrm{~m}, 2 \mathrm{H}), 3.04-3.00(\mathrm{~m}, 2 \mathrm{H}) .{ }^{13} \mathrm{C}$ NMR $\left(151 \mathrm{MHz}\right.$, Methanol- $\left.d_{4}\right) \delta 151.87,151.14,151.00,150.35,124.48,119.35,118.06,116.95,115.90$, $114.83,111.67,110.23,56.01,55.25,55.00,46.63,46.32,26.85$.

Entry 24: N-(4-hydroxy-2-methoxybenzyl)-4-bromo-2,5-dimethoxyphenethylamine hydrochloride<smiles>COc1cc(CCNCc2ccc(O)cc2OC)c(OC)cc1Br</smiles>

This compound was synthesized as previously described. ${ }^{2}$

${ }^{1} \mathrm{H}$ NMR $\left(600 \mathrm{MHz}\right.$, Methanol- $\left.d_{4}\right) \delta 7.23-7.16(\mathrm{~m}, 2 \mathrm{H}), 6.97(\mathrm{~s}, 1 \mathrm{H}), 6.52(\mathrm{~d}, J=2.3 \mathrm{~Hz}, 1 \mathrm{H}), 6.45(\mathrm{dd}, J=8.2$, $2.3 \mathrm{~Hz}, 1 \mathrm{H}$ ), 4.15 (s, 2H), 3.85 (brs, $6 \mathrm{H}$ ), 3.80 (s, 3H), 3.20 (dd, J = 9.0, $6.5 \mathrm{~Hz}, 2 \mathrm{H}$ ), 3.02 (dd, J = 9.0, $6.5 \mathrm{~Hz}$, $2 H) .{ }^{13} \mathrm{C}$ NMR $\left(151 \mathrm{MHz}\right.$, Methanol- $\left.d_{4}\right) \delta 160.60,159.18,151.86,150.31,132.26,124.65,115.84,114.89$, $110.11,109.41,107.09,98.60,56.05,55.28,54.68,46.30,45.99,26.90$. 
Entry 25: N-(2-methoxybenzyl)-glycine methyl ester<smiles>COC(=O)CNCc1ccccc1OC</smiles>

Glycine methyl ester hydrochloride (2.00 g, $15.93 \mathrm{mmol}, 1.00$ eq.), 2-methoxybenzaldehyde (2.17 g, 15.93 $\mathrm{mmol}, 1.00$ eq.) and triethylamine (2.22 mL, $15.93 \mathrm{mmol}, 1.00$ eq.) was dissolved in dry methanol (50 $\mathrm{mL})$ and stirred at reflux under $\mathrm{N}_{2}$ with 3 Ångstrøm molecular sieves overnight. After cooling the reaction mixture to room temperature, $\mathrm{NaCNBH}_{3}(2.00 \mathrm{~g}, 31.86 \mathrm{mmol}, 2.00$ eq.) was added, and the reaction stirred another 24 hours at ambient temperature. The mixture was diluted with EtOAc and filtered through celite, the filtrate was washed with brine, dried over $\mathrm{Na}_{2} \mathrm{SO}_{4}$, filtered and concentrated by rotary evaporation. The residue was purified by flashchromatography (45:5:50-heptane:triethylamine:EtOAc, $\mathrm{Rf}=0.5$ ) yielding the product as a yellow oil (1.23 g, $37 \%)$.

${ }^{1} \mathrm{H}$ NMR (600 MHz, Chloroform-d) $\delta 7.21-7.15(\mathrm{~m}, 2 \mathrm{H}), 6.84(\mathrm{td}, J=7.4,1.1 \mathrm{~Hz}, 1 \mathrm{H}), 6.81-6.78(\mathrm{~m}, 1 \mathrm{H})$, $3.78-3.73(\mathrm{~m}, 5 \mathrm{H}), 3.62(\mathrm{~s}, 3 \mathrm{H}), 3.35(\mathrm{~s}, 2 \mathrm{H}) .{ }^{13} \mathrm{C}$ NMR (151 MHz, Chloroform-d) $\delta 172.51,157.72,130.09$, $128.66,126.93,120.45,110.29,55.29,51.76,49.76,48.46$.

Entry 26: N-BOC-N-(2-methoxybenzyl)-glycine methyl ester<smiles>COC(=O)CN(Cc1ccccc1OC)C(=O)OC(C)(C)C</smiles>

$\mathrm{N}$-(2-methoxybenzyl)-glycine methyl ester (1.00 g, $4.78 \mathrm{mmol}, 1.00$ eq.), (BOC) ${ }_{2} \mathrm{O}$ (1.25 g, $5.73 \mathrm{mmol}, 1.20$ eq.), triethylamine ( $1.33 \mathrm{~mL}, 9.56 \mathrm{mmol}, 2.00$ eq.) and DMAP (0.08 g, $0.07 \mathrm{mmol}, 0.01$ eq.) was stirred in THF (30 mL) overnight. The reaction mixture was concentrated by rotary evaporation, and the residue was purified by flashchromatography ( $10 \%$ EtOAc in heptane, $\mathrm{Rf}=0.2$ ) yielding the product as a colorless oil $(1.20 \mathrm{~g}, 81 \%)$.

${ }^{1} \mathrm{H}$ NMR $(600 \mathrm{MHz}$, Chloroform-d) $\delta 7.25-7.11(\mathrm{~m}, 2 \mathrm{H}), 6.85(\mathrm{t}, J=7.4 \mathrm{~Hz}, 1 \mathrm{H}), 6.78(\mathrm{dd}, J=8.2,4.2 \mathrm{~Hz}, 1 \mathrm{H})$, $4.44(\mathrm{~d}, J=31.5 \mathrm{~Hz}, 2 \mathrm{H}), 3.88(\mathrm{~d}, J=79.2 \mathrm{~Hz}, 2 \mathrm{H}), 3.73(\mathrm{~d}, J=4.7 \mathrm{~Hz}, 3 \mathrm{H}), 3.63(\mathrm{~d}, J=4.9 \mathrm{~Hz}, 3 \mathrm{H}), 1.38(\mathrm{~d}, J=$ 
14.1 Hz, 9H). ${ }^{13} \mathrm{C}$ NMR (151 MHz, Chloroform-d) $\delta$ 170.78, 170.71, 157.59, 157.41, 156.05, 155.45, 130.02, $129.31,128.63,128.58,125.77,125.65,120.62,120.37,110.20,110.17,80.38,80.16,55.23,55.15,51.88$, $51.79,48.82,48.30,46.69,46.07,28.38,28.28$.

Entry 27: N-BOC-N-(2-methoxybenzyl)-glycine<smiles>COc1ccccc1CN(CC(=O)O)C(=O)OC(C)(C)C</smiles>

N-BOC-N-(2-methoxybenzyl)-glycine methyl ester (1.00 g, $3.23 \mathrm{mmol}, 1.00 \mathrm{eq})$, was dissolved in THF (20 $\mathrm{mL}$ ), then $1 \mathrm{M} \mathrm{LiOH} \mathrm{in} \mathrm{H}_{2} \mathrm{O}(20 \mathrm{~mL}, 20 \mathrm{mmol}, 6.19$ eq.) was added and the reaction stirred for 10 minutes. $1 \mathrm{M}$ aq. $\mathrm{HCl}(20 \mathrm{~mL})$ was added, followed by brine. The resulting aq. phase was separated and extracted twice with EtOAc. The combined organic fractions were dried over $\mathrm{MgSO}_{4}$, filtered and concentrated. The residue $(1.10 \mathrm{~g}, 115 \%)$ was used in the following step without further purification.

Entry 28: tert-butyl (2-(methoxy(methyl)amino)-2-oxoethyl)(2-methoxybenzyl)carbamate<smiles>COc1ccccc1CN(CC(=O)N(C)OC)C(=O)OC(C)(C)C</smiles>

N-BOC-N-(2-methoxybenzyl)-glycine (1.10 g, 3.72 mmol, 1.00 eq.), N,O-dimethylhydroxylamine hydrochloride $(0.363 \mathrm{~g}, 3.72 \mathrm{mmol}, 1.00$ eq.) and triethylamine $(2.08 \mathrm{~mL}, 14.90 \mathrm{mmol}, 4.00$ eq.) was dissolved in dry dichloromethane $(30 \mathrm{~mL})$, stirred under $\mathrm{Ar}$ and cooled to $-10{ }^{\circ} \mathrm{C}$. $50 \%$ propylphosphonic acid anhydride in EtOAc ( $3.56 \mathrm{~g}, 5.59 \mathrm{mmol}, 1.50$ eq.) was added dropwise, and the reaction stirred for 30 minutes, before being quenched by addition of sat. aq. bicarbonate $(30 \mathrm{~mL})$. The aq. fraction was separated and extracted twice with dichloromethane. The combined organic fractions were dried over $\mathrm{MgSO}_{4}$, filtered and concentrated by rotary evaporation. The residue was purified by flashchromatography (10\% EtOAc in heptane, $\mathrm{Rf}=0.1)$ yielding the product as a white solid $(1.00 \mathrm{~g}, 79 \%)$.

${ }^{1} \mathrm{H}$ NMR $(600 \mathrm{MHz}$, Chloroform-d) $\delta 7.26-7.13(\mathrm{~m}, 2 \mathrm{H}), 6.85(\mathrm{td}, J=7.4,1.1 \mathrm{~Hz}, 1 \mathrm{H}), 6.77$ (dd, $J=8.1,5.3$ $\mathrm{Hz}, 1 \mathrm{H}), 4.47(\mathrm{~d}, J=27.9 \mathrm{~Hz}, 2 \mathrm{H}), 4.04(\mathrm{~d}, J=77.4 \mathrm{~Hz}, 2 \mathrm{H}), 3.72(\mathrm{~d}, J=6.6 \mathrm{~Hz}, 3 \mathrm{H}), 3.56(\mathrm{~d}, J=26.6 \mathrm{~Hz}, 3 \mathrm{H})$, $3.10(\mathrm{~s}, 3 \mathrm{H}), 1.38(\mathrm{~d}, J=4.6 \mathrm{~Hz}, 9 \mathrm{H}) .{ }^{13} \mathrm{C}$ NMR $(151 \mathrm{MHz}$, Chloroform-d) $\delta 157.60,157.42,156.40,155.85$, 
$129.64,129.00,128.35,128.30,126.29,126.17,120.55,120.30,110.24,110.16,80.00,79.76,61.21,61.15$, $55.26,55.18,47.82,47.48,46.79,46.05,28.41,28.32$.

Entry 29: N-BOC-N-(2-methoxybenzyl)-4-bromo-2,5-dimethoxy-beta-ketophenethylamine<smiles>COc1cc(C(=O)N(CC(=O)OC(C)(C)C)Cc2ccccc2OC)c(OC)cc1Br</smiles>

1,4-dibromo-2,5-dimethoxybenzene ( $0.875 \mathrm{~g}, 2.96 \mathrm{mmol}, 2.00$ eq.) was dissolved in dry THF (10 $\mathrm{mL})$, stirred under $\mathrm{Ar}$ and cooled to $0^{\circ} \mathrm{C}$. A $1.3 \mathrm{M}$ solution of $\mathrm{PrMgCl} \mathrm{LiCl-complex} \mathrm{in} \mathrm{THF} \mathrm{(} 2.50 \mathrm{~mL}, 3.26 \mathrm{mmol}, 2.2$ eq.) was added dropwise, and the reaction stirred for 1 hour. tert-butyl (2-(methoxy(methyl)amino)-2oxoethyl)(2-methoxybenzyl)carbamate $(0.500 \mathrm{~g}, 1.48 \mathrm{mmol}, 1.00$ eq.) was dissolved in THF (5 mL), cooled to $0{ }^{\circ} \mathrm{C}$ and stirred under $\mathrm{Ar}$ before the Grignard reaction mixture was added dropwise. The reaction mixture was stirred another 30 minutes at $0{ }^{\circ} \mathrm{C}$ before the cooling bath was removed and the reaction allowed to stir at ambient temperature overnight. The reaction was quenched by addition of sat. aq. $\mathrm{NH}_{4} \mathrm{Cl}$, and extracted 3 times with EtOAc. The combined organic fractions were washed with brine, dried over $\mathrm{Na}_{2} \mathrm{SO}_{4}$, filtered and concentrated. The residue was purified by flashchromatography (10\% EtOAc in heptane $\mathrm{Rf}=0.3)$, yielding the product as a white solid $(0.510 \mathrm{~g}, 70 \%)$.

${ }^{1} \mathrm{H}$ NMR $(400 \mathrm{MHz}$, Chloroform-d) $\delta 7.22-7.08(\mathrm{~m}, 4 \mathrm{H}), 6.87(\mathrm{t}, J=7.4 \mathrm{~Hz}, 1 \mathrm{H}), 6.76(\mathrm{t}, J=8.1 \mathrm{~Hz}, 1 \mathrm{H}), 4.52$ (d, $J=32.8 \mathrm{~Hz}, 2 \mathrm{H}), 4.43(\mathrm{~d}, J=4.8 \mathrm{~Hz}, 2 \mathrm{H}), 3.82(\mathrm{~d}, J=3.3 \mathrm{~Hz}, 3 \mathrm{H}), 3.76(\mathrm{~d}, J=2.3 \mathrm{~Hz}, 3 \mathrm{H}), 3.66(\mathrm{~d}, J=12.3$ $\mathrm{Hz}, 3 \mathrm{H}), 1.36(\mathrm{~d}, J=27.3 \mathrm{~Hz}, 9 \mathrm{H})$. 
Entry 30: N-BOC-N-(2-methoxybenzyl)-4-bromo-2,5-dimethoxy-beta-hydroxyphenethylamine<smiles>COc1cc(C(O)CN(Cc2ccccc2OC)C(=O)OC(C)(C)C)c(OC)cc1Br</smiles>

N-BOC-N-(2-methoxybenzyl)-4-bromo-2,5-dimethoxy-beta-ketophenethylamine (0.300 g, $0.607 \mathrm{mmol}, 1.00$ eq.) was stirred in $\mathrm{EtOH}(10 \mathrm{~mL}) . \mathrm{NaBH}_{4}(0.100 \mathrm{~g}, 2.64 \mathrm{mmol}, 4.36$ eq.) was added and the mixture stirred for 1 hour, then partitioned between EtOAc $(100 \mathrm{~mL})$ and brine $(100 \mathrm{~mL})$. The aq. phase was separated and extracted twice with EtOAc, the combined organinc fractions were washed with brine, dried over $\mathrm{MgSO}_{4}$, filtered and concentrated by rotary evaporation. The residual colorless oil $(0.240 \mathrm{~g}, 79 \%)$ was used in the following de-protection step without further purification.

Entry 31: N-(2-methoxybenzyl)-4-bromo-2,5-dimethoxy-beta-hydroxyphenethylamine<smiles>COc1cc(C(O)CNCc2ccccc2OC)c(OC)cc1Br</smiles>

N-BOC-N-(2-methoxybenzyl)-4-bromo-2,5-dimethoxy-beta-hydroxyphenethylamine $(0.200 \mathrm{~g}, 0.403 \mathrm{mmol}$, 1.00 eq.) was dissolved in $4 \mathrm{~N} \mathrm{HCl}$ in dioxane ( $5 \mathrm{~mL}, 20 \mathrm{mmol}, 50$ eq.), and stirred for 20 minutes. The reaction mixture was diluted with diethyl ether and placed at $5{ }^{\circ} \mathrm{C}$ over night. The resulting white crystals $(0.090 \mathrm{~g}, 52 \%)$ were collected by decanting off the supernatant, washing with diethyl ether and drying under high vacuum.

${ }^{1} \mathrm{H}$ NMR $\left(600 \mathrm{MHz}, \mathrm{DMSO}-d_{6}\right) \delta 9.16$ (brs, $\left.1 \mathrm{H}\right), 8.89$ (brs, $\left.1 \mathrm{H}\right), 7.48$ (dd, $\left.J=7.5,1.7 \mathrm{~Hz}, 1 \mathrm{H}\right), 7.45-7.40(\mathrm{~m}$, $1 \mathrm{H}), 7.23-7.19(\mathrm{~m}, 2 \mathrm{H}), 7.10(\mathrm{dd}, J=8.4,1.0 \mathrm{~Hz}, 1 \mathrm{H}), 7.01(\mathrm{td}, J=7.4,1.0 \mathrm{~Hz}, 1 \mathrm{H}), 6.26(\mathrm{~d}, J=4.7 \mathrm{~Hz}, 1 \mathrm{H})$, $5.21-5.15(\mathrm{~m}, 1 \mathrm{H}), 4.18(\mathrm{q}, J=13.5 \mathrm{~Hz}, 2 \mathrm{H}), 3.84(\mathrm{~s}, 3 \mathrm{H}), 3.81(\mathrm{~s}, 3 \mathrm{H}), 3.73(\mathrm{~s}, 3 \mathrm{H}), 3.07-3.01(\mathrm{~m}, 1 \mathrm{H}), 2.90$ - 2.82 (m, 1H). ${ }^{13} \mathrm{C}$ NMR $\left(151 \mathrm{MHz}\right.$, DMSO- $\left.d_{6}\right) \delta 158.07,150.13,132.01,131.28,130.37,120.85,119.85$, $116.38,111.58,111.54,110.15,63.32,57.05,56.74,56.09,51.42,45.66,40.54$. 
Entry 32: N-(2-methoxybenzyl)-4-bromo-2,5-dimethoxy-beta-hydroxyphenethylamine<smiles>COc1cc(C(=O)CNCc2ccccc2OC)c(OC)cc1Br</smiles>

The compound was synthesized using the same procedure as for compound entry 29, except using N-BOC$\mathrm{N}$-(2-methoxybenzyl)-4-bromo-2,5-dimethoxy-beta-ketophenethylamine as the starting material.

${ }^{1} \mathrm{H}$ NMR $\left(400 \mathrm{MHz}, \mathrm{DMSO}-d_{6}\right) \delta 9.33(\mathrm{~s}, 2 \mathrm{H}), 7.59(\mathrm{~s}, 1 \mathrm{H}), 7.49(\mathrm{dd}, J=7.4,1.6 \mathrm{~Hz}, 1 \mathrm{H}), 7.47-7.40(\mathrm{~m}, 2 \mathrm{H})$, $7.10(\mathrm{~d}, J=8.3 \mathrm{~Hz}, 1 \mathrm{H}), 7.02(\mathrm{t}, J=7.4 \mathrm{~Hz}, 1 \mathrm{H}), 4.41(\mathrm{~s}, 2 \mathrm{H}), 4.21(\mathrm{~s}, 2 \mathrm{H}), 3.91(\mathrm{~s}, 3 \mathrm{H}), 3.87(\mathrm{~s}, 3 \mathrm{H}), 3.81(\mathrm{~s}$, 3H). ${ }^{13} \mathrm{C}$ NMR $\left(101 \mathrm{MHz}\right.$, DMSO- $\left.d_{6}\right) \delta 190.79,158.17,154.52,150.25,132.29,131.45,123.06,120.93$, $119.90,119.86,118.97,112.19,111.57,57.50,57.16,56.03,55.59,45.37$.

Entry 33: N-nitrone-N-(2-methoxybenzyl)-4-bromo-2,5-dimethoxyphenethylamine<smiles>COc1cc(CC[N+]([O-])=Cc2ccccc2OC)c(OC)cc1Br</smiles>

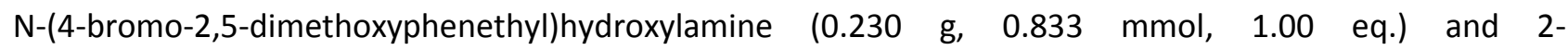
methoxybenzaldehyde $\left(0.118 \mathrm{~g}, 0.867 \mathrm{mmol}, 1.04\right.$ eq.) was dissolved in $\mathrm{EtOH}(10 \mathrm{~mL})$ and heated to $50{ }^{\circ} \mathrm{C}$ for 3 hours. The reaction mixture was concentrated by rotary evaporation and chased with toluene. The residue was purified by flashchromatography (diethyl ether, $\mathrm{Rf}=0.8$ ), yielding the product as white crystals (0.132 g, $40 \%)$.

${ }^{1} \mathrm{H}$ NMR $(600 \mathrm{MHz}$, Chloroform-d) $\delta 9.17(\mathrm{dd}, J=7.9,1.7 \mathrm{~Hz}, 1 \mathrm{H}), 7.44(\mathrm{~s}, 1 \mathrm{H}), 7.29-7.23(\mathrm{~m}, 1 \mathrm{H}), 6.97(\mathrm{~s}$, 1H), $6.95-6.90(\mathrm{~m}, 1 \mathrm{H}), 6.75(\mathrm{dd}, J=8.3,1.0 \mathrm{~Hz}, 1 \mathrm{H}), 6.67(\mathrm{~s}, 1 \mathrm{H}), 4.00(\mathrm{t}, J=6.7 \mathrm{~Hz}, 2 \mathrm{H}), 3.73(\mathrm{~s}, 3 \mathrm{H}), 3.69$ $(\mathrm{s}, 3 \mathrm{H}), 3.54(\mathrm{~s}, 3 \mathrm{H}), 3.19(\mathrm{t}, J=6.7 \mathrm{~Hz}, 2 \mathrm{H}) .{ }^{13} \mathrm{C}$ NMR (151 MHz, Chloroform-d) $\delta$ 156.84, 151.54, 149.86, 131.46, 129.38, 128.59, 126.23, 120.61, 119.36, 115.73, 115.13, 109.85, 109.51, 67.05, 56.69, 56.11, 55.55, 28.91. 
Entry 34: N-hydroxy-N-(2-methoxybenzyl)-4-bromo-2,5-dimethoxyphenethylamine<smiles>COc1cc(CCN(O)Cc2ccccc2OC)c(OC)cc1Br</smiles>

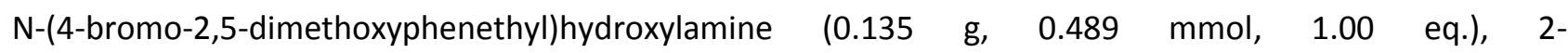
methoxybenzaldehyde (0.105 g, $0.771 \mathrm{mmol}, 1.58$ eq.) and triethylamine $(0.08 \mathrm{~mL}, 0.574 \mathrm{mmol}, 1.17$ eq.) was dissolved in EtOH $(10 \mathrm{~mL})$ and heated at reflux overnight. After cooling to RT the reaction was quenched with $\mathrm{MeOH}$ and concentrated by rotary evaporation. The residue was purified by flashchromatography (diethyl ether, $\mathrm{Rf}=0.7)$ yielding the product as a white solid $(0.050 \mathrm{~g}, 26 \%$ ). ${ }^{1} \mathrm{H}$ NMR $(600 \mathrm{MHz}$, Chloroform-d) $\delta 7.22(\mathrm{dd}, J=7.4,1.7 \mathrm{~Hz}, 1 \mathrm{H}), 7.15(\mathrm{td}, J=7.8,1.8 \mathrm{~Hz}, 1 \mathrm{H}), 6.92(\mathrm{~s}, 1 \mathrm{H})$, $6.82(\mathrm{td}, J=7.4,1.1 \mathrm{~Hz}, 1 \mathrm{H}), 6.78(\mathrm{dd}, J=8.2,1.1 \mathrm{~Hz}, 1 \mathrm{H}), 6.66(\mathrm{~s}, 1 \mathrm{H}), 6.21(\mathrm{~s}, 1 \mathrm{H}), 3.79(\mathrm{~s}, 2 \mathrm{H}), 3.74(\mathrm{~s}, 3 \mathrm{H})$, $3.73(\mathrm{~s}, 3 \mathrm{H}), 3.66(\mathrm{~s}, 3 \mathrm{H}), 2.87-2.76(\mathrm{~m}, 4 \mathrm{H}) .{ }^{13} \mathrm{C}$ NMR (151 MHz, Chloroform-d) $\delta$ 157.84, 152.16, 149.91, 131.34, 129.08, 128.59, 125.51, 120.28, 115.79, 114.90, 110.51, 108.68, 59.50, 58.58, 56.97, 56.14, 55.43, 28.27 .

Entry 35: N-(4-bromo-2,5-dimethoxyphenethyl)hydroxylamine<smiles>COc1cc(CCNO)c(OC)cc1Br</smiles>

4-bromo-2,5-dimethoxyphenethyl methanesulfonate $\left(1.731 \mathrm{~g}, 5.10 \mathrm{mmol}, 1.00\right.$ eq.) and $50 \% \mathrm{NH}_{2} \mathrm{OH}$ in water (1.5 mL, $22.71 \mathrm{mmol}, 4.45$ eq.) was dissolved in $\mathrm{MeOH}(30 \mathrm{~mL}$ ) and heated at reflux overnight. The reaction mixture was concentrated by rotary evaporation and the residue was purified by flash chromatography (50\% EtOAc in heptane, $\mathrm{Rf}=0.5)$, yielding the product as a white solid $(0.377 \mathrm{~g}, 27 \%$ ). ${ }^{1} \mathrm{H}$ NMR (600 MHz, Chloroform-d) $\delta 6.96(\mathrm{~s}, 1 \mathrm{H}), 6.70(\mathrm{~s}, 1 \mathrm{H}), 3.77(\mathrm{~s}, 3 \mathrm{H}), 3.70(\mathrm{~s}, 3 \mathrm{H}), 3.07(\mathrm{t}, J=6.9 \mathrm{~Hz}$, 2H), $2.78(\mathrm{t}, J=6.9 \mathrm{~Hz}, 2 \mathrm{H}) .{ }^{13} \mathrm{C}$ NMR $(151 \mathrm{MHz}$, Chloroform-d) $\delta 151.04,148.95,126.87,114.91,114.03$, $108.14,55.96,55.11,52.33,27.17$. 
Entry 36: 4-bromo-2,5-dimethoxyphenethyl methanesulfonate<smiles>COc1cc(CCOS(C)(=O)=O)c(OC)cc1Br</smiles>

2-(4-bromo-2,5-dimethoxyphenyl)ethan-1-ol (1.098 g, $4.21 \mathrm{mmol}, 1.00$ eq.) and triethylamine $(1.00 \mathrm{~mL}$, $7.17 \mathrm{mmol}, 1.70$ eq.) was dissolved in dry DCM and stirred under $\mathrm{N}_{2}$ at $0{ }^{\circ} \mathrm{C}$. Methanesulfonyl chloride (1.00 $\mathrm{mL}, 12.92 \mathrm{mmol}, 3.07$ eq.) was added dropwise, and the reaction stirred for 15 minutes. The reaction was quenched with sat. aq. bicarb., separated and the aq. phase extracted twice with DCM. The combined organic fractions were dried over $\mathrm{Na}_{2} \mathrm{SO}_{4}$, filtered and concentrated by rotary evaporation. The crude material (white solid, $1.731 \mathrm{~g}, 121 \%$ ) was used in the following step without further purification.

${ }^{1} \mathrm{H}$ NMR $(600 \mathrm{MHz}$, Chloroform-d) $\delta 7.08(\mathrm{~s}, 1 \mathrm{H}), 6.80(\mathrm{~s}, 1 \mathrm{H}), 4.42(\mathrm{t}, J=6.9 \mathrm{~Hz}, 2 \mathrm{H}), 3.87(\mathrm{~s}, 3 \mathrm{H}), 3.82(\mathrm{~s}$, $3 \mathrm{H}), 3.05(\mathrm{t}, J=6.9 \mathrm{~Hz}, 2 \mathrm{H}), 2.92(\mathrm{~s}, 3 \mathrm{H}) .{ }^{13} \mathrm{C}$ NMR (151 MHz, Chloroform-d) $\delta 151.89,150.00,124.49$, $115.89,115.41,110.21,68.70,57.03,56.07,37.38,30.94$.

Entry 37: 2-(4-bromo-2,5-dimethoxyphenyl)ethan-1-ol<smiles>COc1cc(CCO)c(OC)cc1Br</smiles>

4-bromo-2,5-dimethoxyphenyl acetic acid (4.00 g, $14.54 \mathrm{mmol}, 1.00$ eq.) was dissolved in THF (50 mL). Neat borane dimethylsulphide complex $(2.65 \mathrm{~mL}, 43.62 \mathrm{mmol}, 3.00 \mathrm{eq})$ was added dropwise and the solution stirred under $\mathrm{N}_{2}$ overnight. The reaction was then cooled with an icebath and carefully quenched by dropwise addition of $20 \mathrm{~mL} 2 \mathrm{~N} \mathrm{HCl}$ in $\mathrm{MeOH}$, removal of cooling bath and stirring at ambient temperature for an additional 1 hour. Concentration by rotary evaporation and chasing with $\mathrm{MeOH}$ gave a clear oil. $3 \mathrm{~mL}$ toluene and a seed crystal was added to the residue, resulting in the precipitation of the title compound as white crystals ( $2.98 \mathrm{~g}, 78 \%)$.

${ }^{1} \mathrm{H}$ NMR (600 MHz, Chloroform-d) $\delta 7.07(\mathrm{~s}, 1 \mathrm{H}), 6.80(\mathrm{~s}, 1 \mathrm{H}), 3.87(\mathrm{~s}, 3 \mathrm{H}), 3.84(\mathrm{t}, J=6.4 \mathrm{~Hz}, 2 \mathrm{H}), 3.81(\mathrm{~s}$, 3H), $2.88(\mathrm{t}, J=6.4 \mathrm{~Hz}, 2 \mathrm{H}) .{ }^{13} \mathrm{C}$ NMR $(151 \mathrm{MHz}$, Chloroform-d) $\delta 152.03,150.01,127.33,115.97,115.32$, $109.34,62.53,56.97,56.15,34.13$. 
Entry 38: 4-bromo-2,5-dimethoxyphenyl acetic acid<smiles>COc1cc(CC(=O)O)c(OC)cc1Br</smiles>

2,5-dimethoxyhenyl acetic acid (4.927 g, $25.11 \mathrm{mmol}$, 1.00 eq.) was suspended in $\mathrm{AcOH}$ ( $25 \mathrm{~mL}) . \mathrm{Br}_{2}(0.68$ $\mathrm{mL}, 26.37 \mathrm{mmol}, 1.05$ eq.) dissolved in $\mathrm{AcOH}$ ( $25 \mathrm{~mL}$ ) was added dropwise to the suspension under $\mathrm{N}_{2}$. The reaction was stirred overnight before being poured into cold water $(200 \mathrm{~mL})$ with stirring. The resulting white precipitate was filtered and recrystallized from iPA and water (white crystals, $3.13 \mathrm{~g}, 45 \%$ ).

${ }^{1} \mathrm{H}$ NMR $\left(600 \mathrm{MHz}\right.$, Chloroform-d) $\delta 7.10(\mathrm{~s}, 1 \mathrm{H}), 6.82(\mathrm{~s}, 1 \mathrm{H}), 3.87(\mathrm{~s}, 3 \mathrm{H}), 3.81(\mathrm{~s}, 3 \mathrm{H}), 3.66(\mathrm{~s}, 2 \mathrm{H}) .{ }^{13} \mathrm{C}$ NMR (151 MHz, Chloroform-d) $\delta 176.94,151.88,150.01,122.20,116.26,115.25,110.79,56.96,56.35,35.56$.

\section{Radio chemistry}

General Procedures

\section{$\left[{ }^{11} \mathrm{C}\right]$ methyl trifluoromethylsuphonate (methyl triflate)}

$\left[{ }^{11} \mathrm{C}\right]$ methane was prepared "in-target" by $16 \mathrm{MeV}$ proton bombardment of a pressurised gas mixture of $10 \%$ hydrogen in nitrogen (25 bar) using using the Scanditronix MC-32 cyclotron at Copenhagen University Hospital Rigshospitalet. Subsequently the radioactive gas was transferred to an automated radiochemistry system, where the $\left[{ }^{11} \mathrm{C}\right]$ methane was trapped on a small, cooled column (Haysep $\mathrm{Q},-160 \stackrel{\circ}{ } \mathrm{C}$ ). ${ }^{3}$ Following complete trapping, the column was heated to $\mathrm{X} \stackrel{\mathrm{O}}{\mathrm{C}}$ to release the radioactivity to a recirculating system, where the a high temperature reaction with iodine coverted it to $\left[{ }^{11} \mathrm{C}\right]$ iodomethane (methyl iodide), which was trapped on the same Haysep $Q$ column at $X{ }^{\circ} C^{4}{ }^{4}$ Heating the Haysep $Q$ column to $100 \stackrel{\circ}{ }$ a a flow of helium gas $\left(30 \mathrm{ml} / \mathrm{min}\right.$ ) allowed for release of the formed $\left[{ }^{11} \mathrm{C}\right]$ iodomethane, which was coverted online to $\left[{ }^{11} \mathrm{C}\right]$ methyl triflate by passing the gas stream through a small column containing silver trifluoromethylsuphonate absorbed on an inert carbon support and heated to $180 \stackrel{\circ}{ } \mathrm{C}$. The resultant $\left[{ }^{11} \mathrm{C}\right]$ methyl triflate was trapped in a $1.1 \mathrm{ml}$ vial containing a $300 \mu \mathrm{l}$ solution of the appropriate radiolabelling precursor (see below for details). 


\section{Preparative HPLC}

All $\left[{ }^{11} \mathrm{C}\right]$-labelled compounds were purified using the same method using a Knaur HPLC system and a Waters Xterra C18, $2.5 \mu \mathrm{m}, 50 \times 10 \mathrm{~mm}$ column; temperature $60^{\circ} \mathrm{C}$; eluent $25 / 75$ ethanol/0.082\% phosphoric acid; flow rate $9 \mathrm{ml} / \mathrm{min}$.

\section{Analytical HPLC}

Analysis of final products was performed using a Ultimate 3000 HPLC system (Thermo Scientific) with a Kinetex C-18, 4.6 x 50 mm column (Phenomenex) eluted with 33/67 acetonitrile/0.082\% phosphoric acid; flow rate $1.5 \mathrm{ml} / \mathrm{min}$, with in-line UV $(296 \mathrm{~nm})$ and radiodetection.<smiles>COc1cc(CCN(Cc2ccccc2O)C(=O)OC(C)(C)C)c(OC)cc1Br</smiles><smiles>COc1cc(CCNCc2ccccc2OC)c(OC)cc1Br</smiles>

The compound was prepared as previously described with minor modifications. ${ }^{5}$ Briefly, $\left[{ }^{11} \mathrm{C}\right] \mathrm{methyl}$ triflate was transferred in a stream of helium to a $1.1 \mathrm{ml}$ vial containing $0.1-0.2 \mathrm{mg}$ of the labelling precursor (tertbutyl 4-bromo-2,5-dimethoxyphenethyl(2-hydroxybenzyl)carbamate) and $2 \mu \mathrm{l} 2 \mathrm{M} \mathrm{NaOH}$ in $300 \mu \mathrm{l}$ acetone, cooled to $-10^{\circ} \mathrm{C}$. The resulting mixture was heated at $40^{\circ} \mathrm{C}$ for 30 seconds to allow for complete reaction of the $\left[{ }^{11} \mathrm{C}\right]$ methyl triflate followed by addition of $250 \mu \mathrm{lTFA} /$ acetone $(1: 1)$ and heating at $80^{\circ} \mathrm{C}$ for $5 \mathrm{~min}$ to hydrolyse the boc protecting group. After neutralization with a mixture of $750 \mu \mathrm{l} 2 \mathrm{M} \mathrm{NaOH}$ and $3.7 \mathrm{ml}$ $0.082 \%$ phosphoric acid, the reaction mixture was purified by HPLC. The fraction corresponding to the radiolabelled product (retention time $3.7 \mathrm{~min}$ ) was collected by allowing the HPLC eluent to flow directly through a $0.22 \mu \mathrm{m}$ sterile filter (Millex GV, Millipore) into a $20 \mathrm{ml}$ glass vial containing $9 \mathrm{ml}$ sterile phosphate buffered saline solution ( $\mathrm{pH} 7$ ), giving a $14 \mathrm{ml}$ sterile solution of the labelled product containing around $7 \% \mathrm{w} / \mathrm{v}$ ethanol. The total synthesis time after end of bombardment was approx. $30 \mathrm{~min}$, yielding 1.0-3.5 GBq of product with a radiochemical purity of $>99 \%$ and specific radioactivity in the range of $240-$ $1400 \mathrm{GBq} / \mu \mathrm{mol}$ at the end of synthesis. 
<smiles>COc1cc(CCN(Cc2ccccc2OC)C(=O)OC(C)(C)C)c(O)cc1CCNCc1ccccc1OC</smiles>

This compound was prepared exactly as described above but using (tert-butyl 4-bromo-2-hydroxy,5methoxyphenethyl(2-methoxybenzyl)carbamate) as radiolabelling precursor, yielding $1.8 \mathrm{GBq}$ of product with a radiochemical purity of $>97 \%$ and specific radioactivity of $445 \mathrm{GBq} / \mu \mathrm{mol}$ at the end of synthesis.<smiles>COc1cc(Br)c(O)cc1CCNCc1ccccc1OC</smiles><smiles>COc1cc(CCNCc2ccccc2OC)c(OC)cc1Br</smiles>

This compound was prepared by selective $\left[{ }^{11} \mathrm{C}\right]$-methylation $\left(\left[{ }^{11} \mathrm{C}\right]\right.$ methyl triflate) of the corresponding phenol precursor (entry 20) without protection of the secondary amine moiety, yielding 2.0-3.5 GBq of product with a radiochemical purity of $>97 \%$ and specific radioactivity of $500-1000 \mathrm{GBq} / \mu \mathrm{mol}$ at the end of synthesis. To improve $O$ vs. $N$ selectivity (ratio - 60:40) the precursor solution (1 mg precursor in $300 \mu \mathrm{l}$ acetone $+1 \mu \mathrm{l} 2 \mathrm{M}$ aq. $\mathrm{NaOH}$ ) was heated at $40^{\circ} \mathrm{C}$ for 20 minutes, then cooled to $-10^{\circ} \mathrm{C}$ prior to trapping of the $\left[{ }^{11} \mathrm{C}\right]$ methyl triflate.<smiles>CCOc1ccccc1C=O</smiles><smiles>COc1cc(Br)c(O)cc1CCN</smiles>

Reductive Amination<smiles>CCOc1ccccc1CNCCc1cc(O)c(Br)cc1OC</smiles>

This compound was prepared by first reductive amination of $2-\left[{ }^{11} \mathrm{C}\right]$ methoxybenzaldehyde with 2methoxy,4-bromo,5-hydroxyphenethylamine (previously published procedure), ${ }^{6}$ followed by HPLC purification as described above, yielding $384 \mathrm{MBq}$ of product with a radiochemical purity of $>97 \%$ and specific radioactivity of $>1000 \mathrm{GBq} / \mu \mathrm{mol}$ at the end of synthesis. 


\section{Radioactive Metabolite Analysis}

Arterial blood samples from PET studies were analysed using a column-switching HPLC system as previously described but with some modifications. ${ }^{7}$ Briefly, plasma samples were obtained from blood samples by centrifugation and filtered using $0.45 \mu \mathrm{m}$ syringe filter prior to HPLC analysis (Ultimate 3000 system, Thermo Scientific, with an on-line radiodetector, Posi-RAM, LabLogic, UK). Extraction of the lipophilic component of the plasma was achieved using a Shim-pack MAYI-ODS(G) column (30 x $4.6 \mathrm{~mm}, 50 \mu \mathrm{m}$ particles, Shimadzu) with a mobile phase consisting of $20 \mathrm{mM}$ sodium dihydrogen phosphate and 2-mM sodium-1-decane sulphonate (adjusted to pH 7.2 with phosphoric acid) and 2\% 2-propanol at a flow rate of $5 \mathrm{ml} / \mathrm{min}$. After a 4 minute extraction step the extraction column was eluted (reverse flow direction) with $30 \%$ acetonitrile in $100 \mathrm{mM}$ disodium hydrogen phosphate $(\mathrm{pH} 2.6)$, containing $5 \mathrm{mM}$ sodium-1-decane suphonate, flow rate $5 \mathrm{ml} / \mathrm{min}$, through an analytical column (Onyx Monolithic C-18, $50 \times 4.6 \mathrm{~mm}$, Phenomenex). The total analysis time was 8 minutes per sample and up to $4 \mathrm{ml}$ plasma could be analysed per injection.

\section{Animal Studies}

Four female Danish Landrace pigs (mean weight \pm SD, $21 \pm 1.5 \mathrm{~kg}$ ) were used for in vivo PET imaging. The animals were housed under standard conditions and were allowed to acclimatize for 1 week. Before scanning, anaesthesia was induced with i.m. injection of $0.13 \mathrm{~mL} / \mathrm{kg}$ Zoletil veterinary mixture (11.36 $\mathrm{mg} / \mathrm{mL}$ xylazine $+11.36 \mathrm{mg} / \mathrm{mL}$ ketamine $+1.82 \mathrm{mg} / \mathrm{mL}$ butorphanol $+1.82 \mathrm{mg} / \mathrm{mL}$ methadone). Hereafter, anaesthesia was maintained with constant propofol infusion $(15 \mathrm{mg} / \mathrm{kg} / \mathrm{h}$ intravenous (i.v.); B. Braun, Melsungen, Germany). Arterial i.v. access for blood drawing was granted in the right femoral artery via a minor incision and two venous i.v. accesses for injections were granted in the left and right mammary veins Analgesia was assured by i.v. injection of fentanyl during surgery. During anaesthesia, animals were endotracheally intubated and ventilated. Vital parameters (heart rate, body temperature, blood pressure, oxygen saturation and end tidal $\mathrm{CO}_{2}$ ) were continuously monitored during the scan. The pigs were euthanized immediately after scanning with an i.v. injection of pentobarbital $(200 \mathrm{mg} / \mathrm{ml}$ pentobarbital + $20 \mathrm{mg} / \mathrm{ml}$ lidocain hydrochlorid). All animal procedures were approved by the Danish Council for Animal Ethics (journal no. 2012-15-2934-00156).

All tracers were given as i.v. injections. Injected radioactive and mass doses were as follows: 11: 494 and $492 \mathrm{MBq}(\mathrm{n}=2), 0.59$ and 2000.18 $\mu \mathrm{g}(\mathrm{n}=2) ; 12: 518 \mathrm{MBq}, 0.67 \mu \mathrm{g} ; 13: 469 \mathrm{MBq}, 0.71 \mu \mathrm{g} ; 14: 277 \mathrm{MBq}, 0.03$ $\mu \mathrm{g}$. The pigs were scanned for $90 \mathrm{~min}$ in list mode in a high resolution research tomography (HRRT) scanner (Siemens). PET-scanning was started at the time of injection $(\mathrm{t}=0 \mathrm{~min})$. Blood samples were drawn at time 
points $2.5,5,10,20,30,60,90$ minutes. The HRRT list mode data were reconstructed and analyzed as previously described. ${ }^{5}$

\section{Human studies}

3 healthy females (age $22 \pm 2$, mean \pm S.D.) underwent both 11 and 13 PET scans on the same experimental day. Both tracers were given as i.v. injections. Injected radioactive and mass doses were as follows (mean \pm S.D.): $11: 552 \pm 58 \mathrm{MBq}, 0.487 \pm 0.146 \mu \mathrm{g} ; 13: 498 \pm 77 \mathrm{MBq}, 0.333 \pm 0.111 \mu \mathrm{g}$. The subjects were scanned for $120 \mathrm{~min}$ in list mode in a high resolution research tomography (HRRT) scanner (Siemens). Blood samples were drawn throughout the duration of the two scans. The study was registered and approved by the local ethics committee (protocol-ID: (KF) 01 2006-20). All participants gave written informed consent.

\section{References}

(1) Pedersen, D. S., and Rosenbohm, C. (2001) Dry Column Vacuum Chromatography. Synthesis 16, 24312434.

(2) Hansen, M., Phonekeo, K., Paine, J. S., Leth-Petersen, S., Begtrup, M., Brauner-Osborne, H., and Kristensen, J. L. (2014) Synthesis and structure-activity relationships of N-benzyl phenethylamines as 5HT2A/2C agonists. ACS Chem. Neurosci. 5, 243-249.

(3) Koziorowski, J., Larsen, P., and Gillings, N. (2010) A quartz-lined carbon-11 target: striving for increased yield and specific activity. Nucl. Med. Biol. 37, 943-948.

(4) Gillings, N., and P. Larsen, P. (2005) A highly flexible modular radiochemistry system. J. Label Compd. Radiopharm. 48, 338.

(5) Ettrup, A., Hansen, M., Santini, M. A., Paine, J., Gillings, N., Palner, M., Lehel, S., Herth, M. M., Madsen, J., Kristensen, J., Begtrup, M., and Knudsen, G. M. (2011) Radiosynthesis and in vivo evaluation of a series of substituted 11C-phenethylamines as 5-HT (2A) agonist PET tracers. Eur. J. Nucl. Med. Mol. Imaging 38, 681693.

(6) Herth, M. M., Leth-Petersen, S., Lehel, S., Hansen, M., Knudsen, G. M., Gillings, N., Madsen, J., and Kristensen, J. L. (2014) Accelerating preclinical PET-screening: reductive amination with [11C]methoxybenzaldehydes. RSC Advances 4, 21347-21350.

(7) Gillings, N. (2009) A restricted access material for rapid analysis of [11C]-labeled radiopharmaceuticals and their metabolites in plasma. Nucl. Med. Biol. 36, 961-965. 
Analytical Spectra

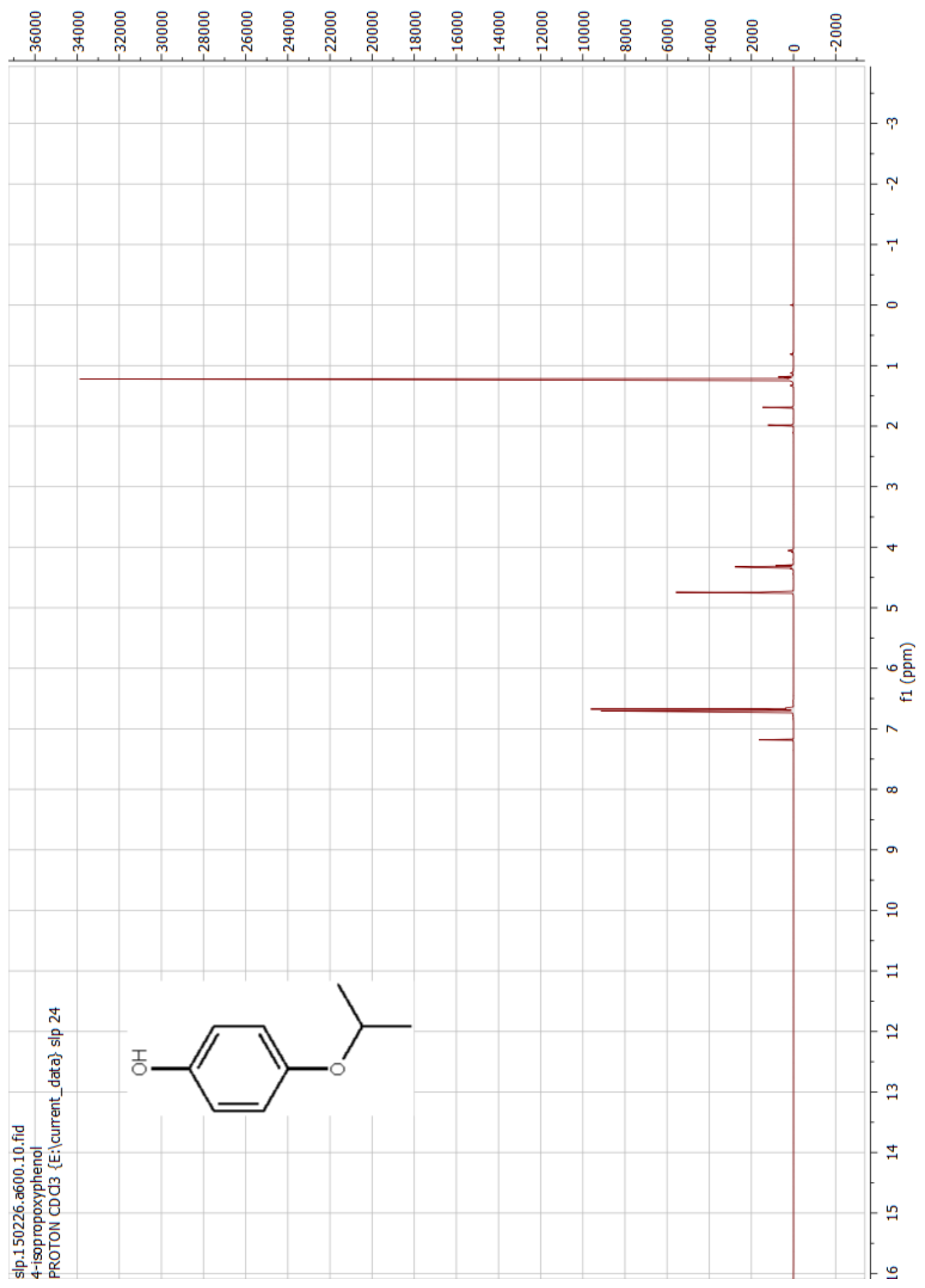




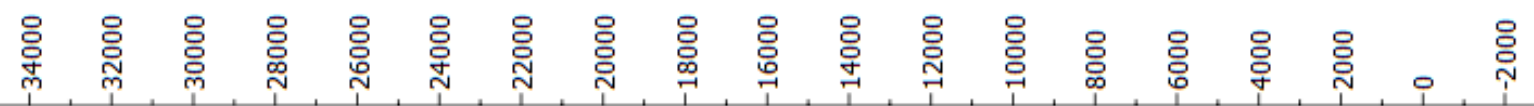
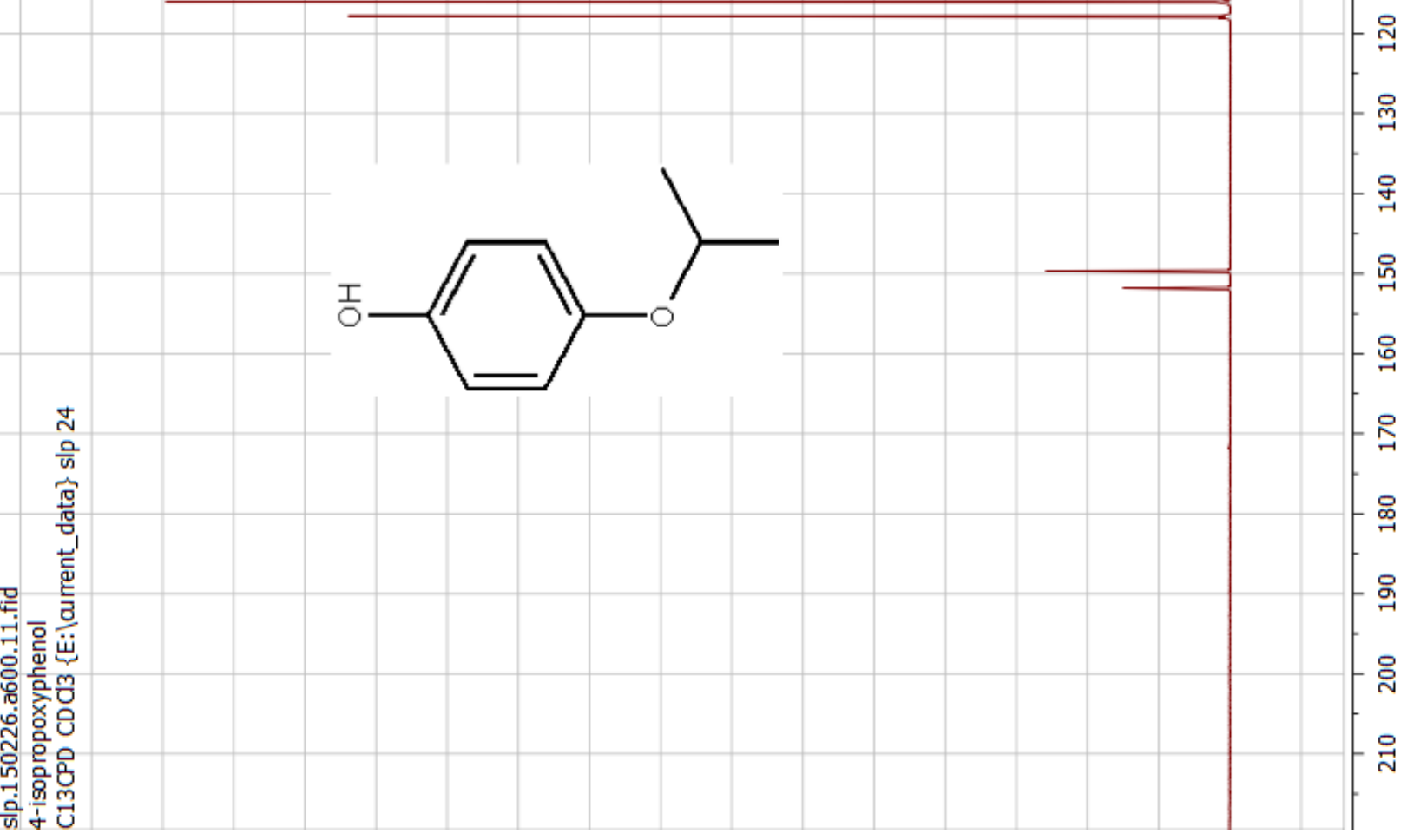


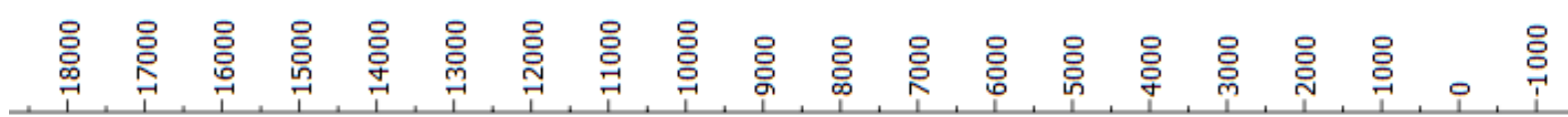

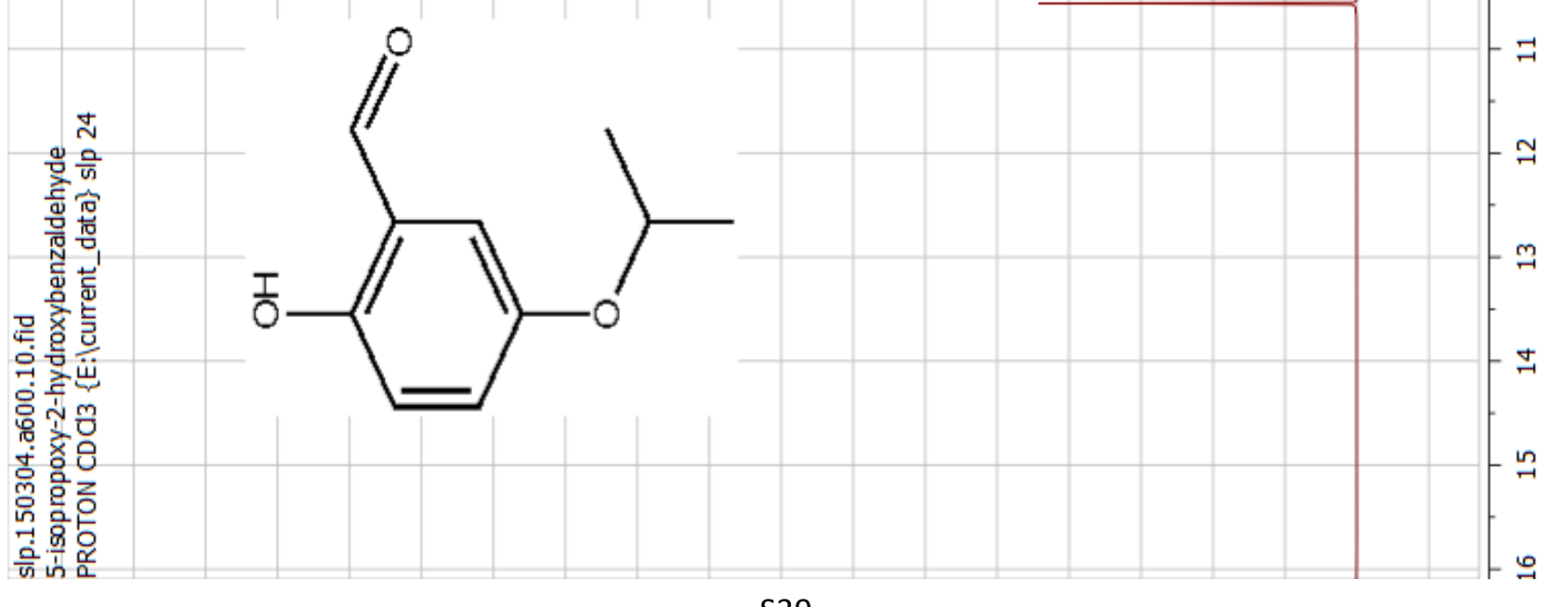




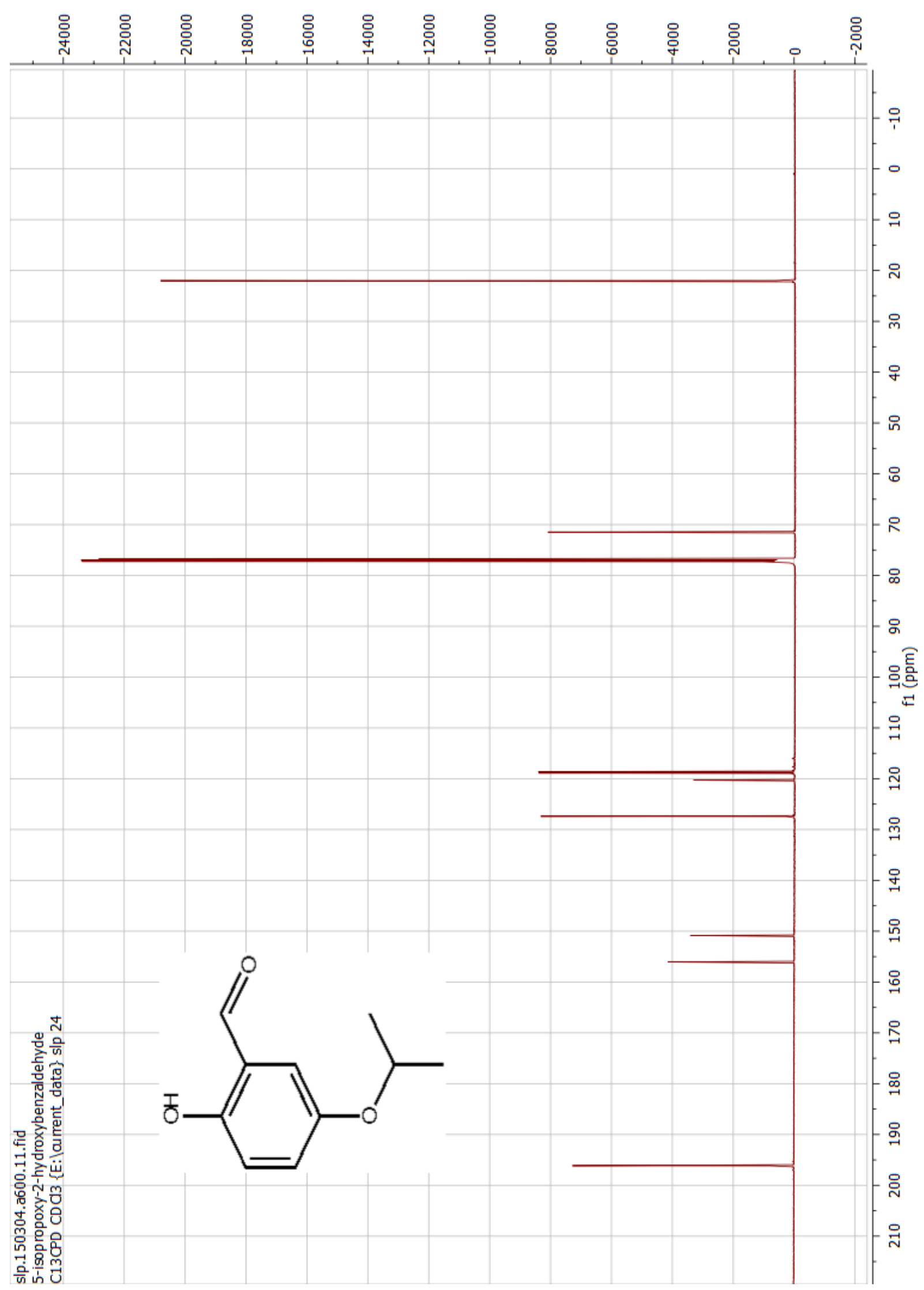




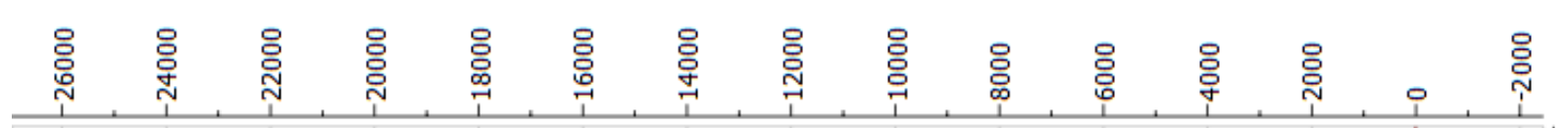

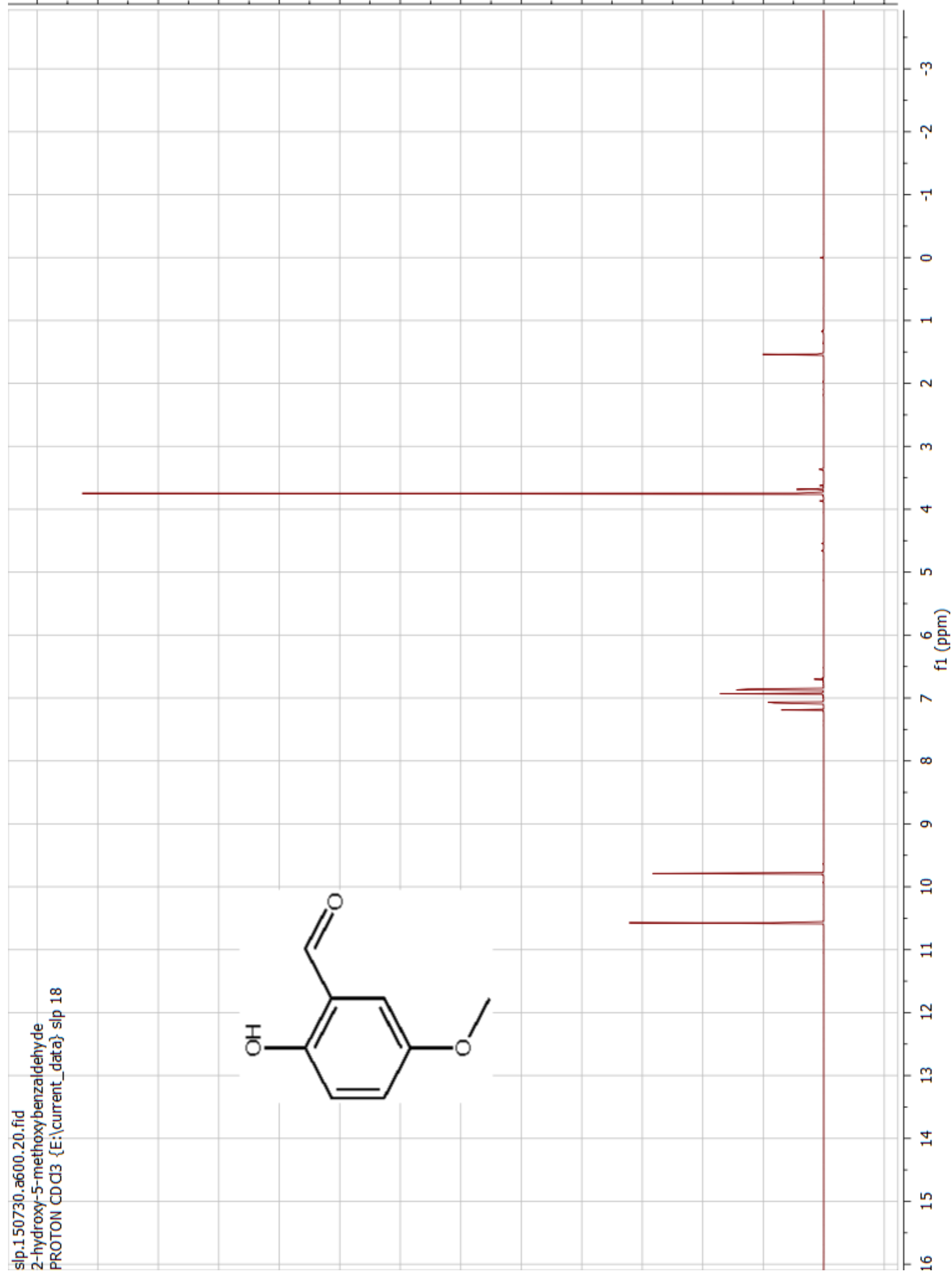




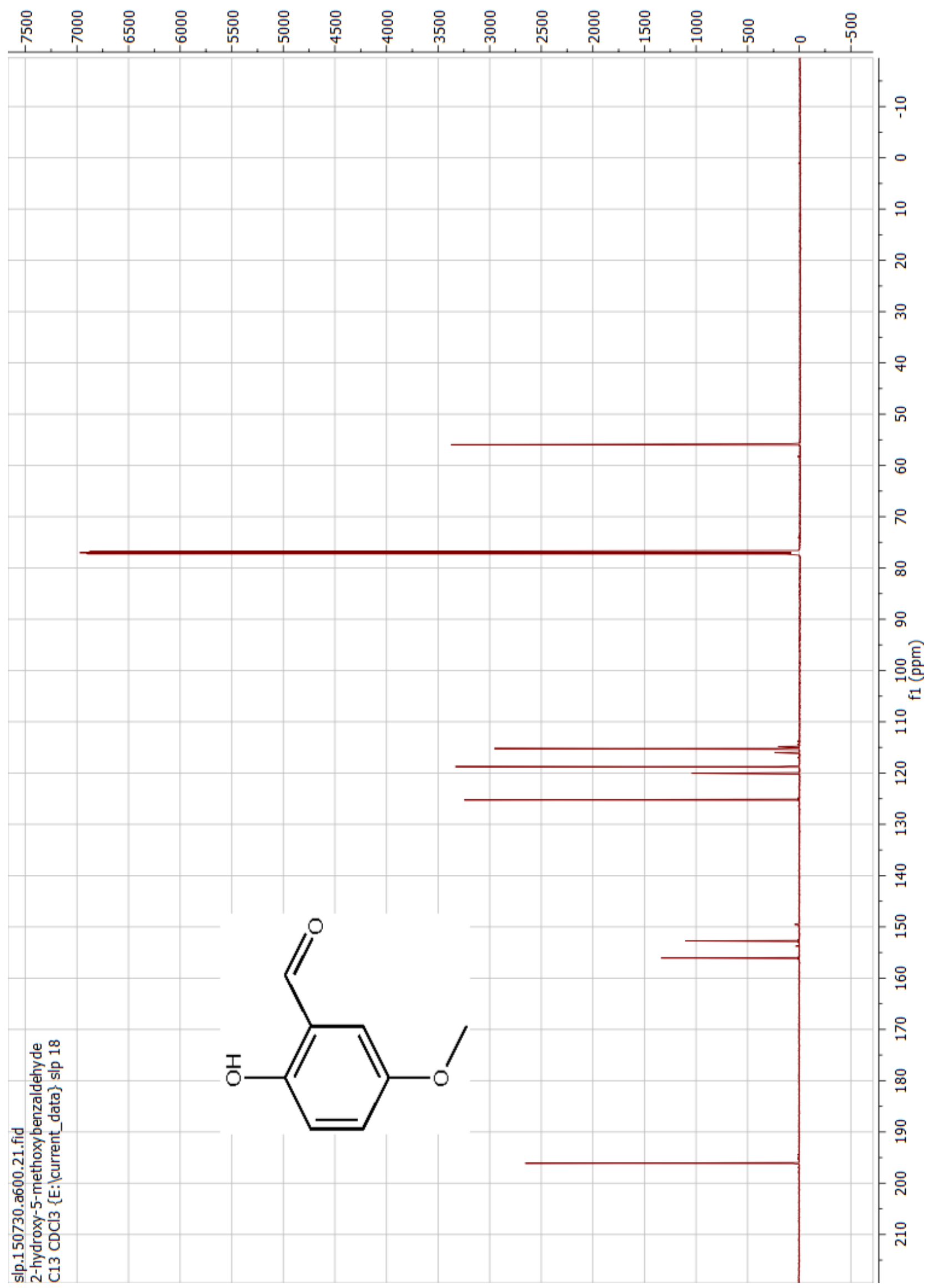




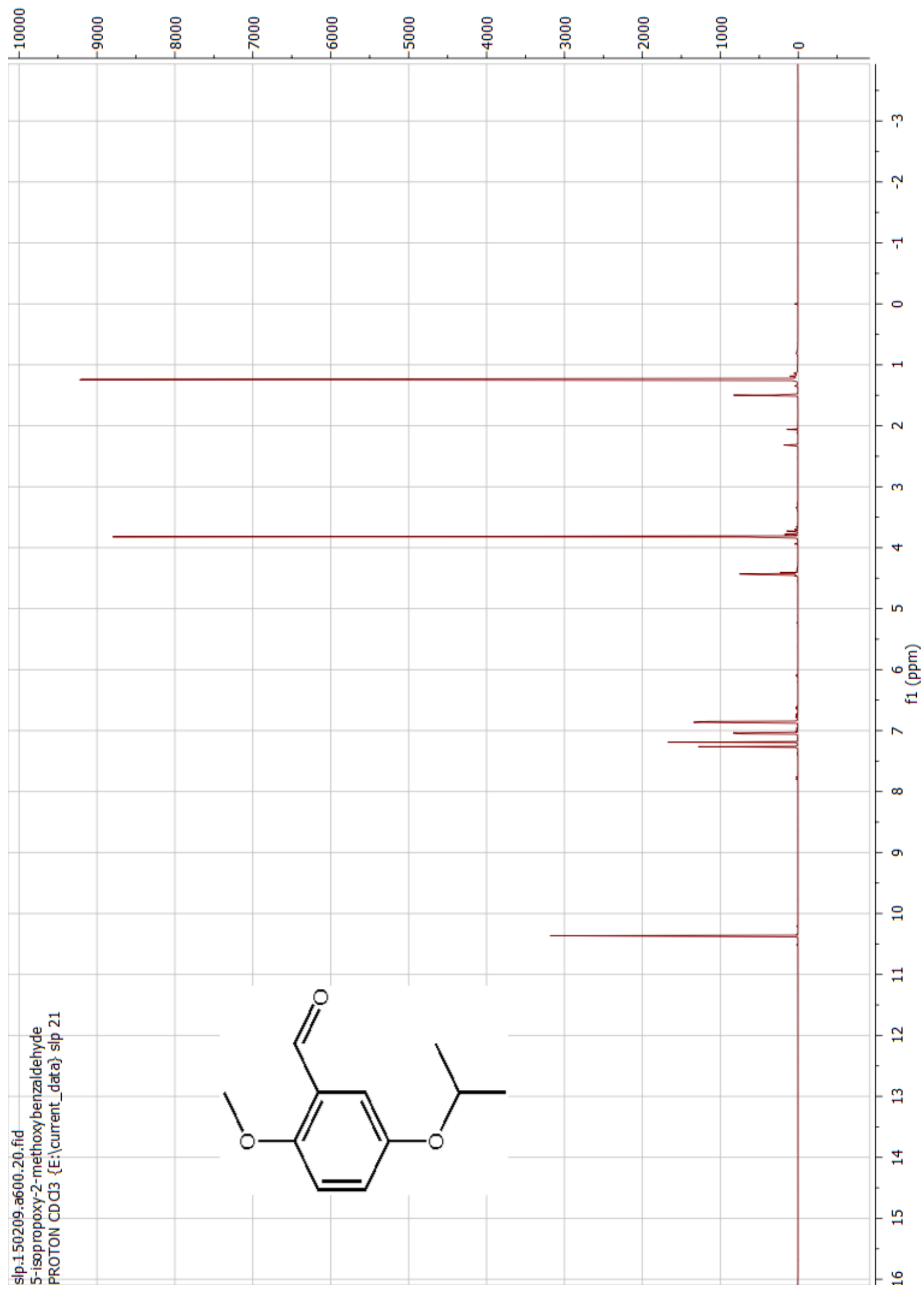




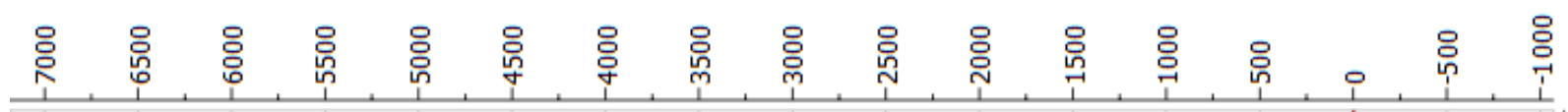

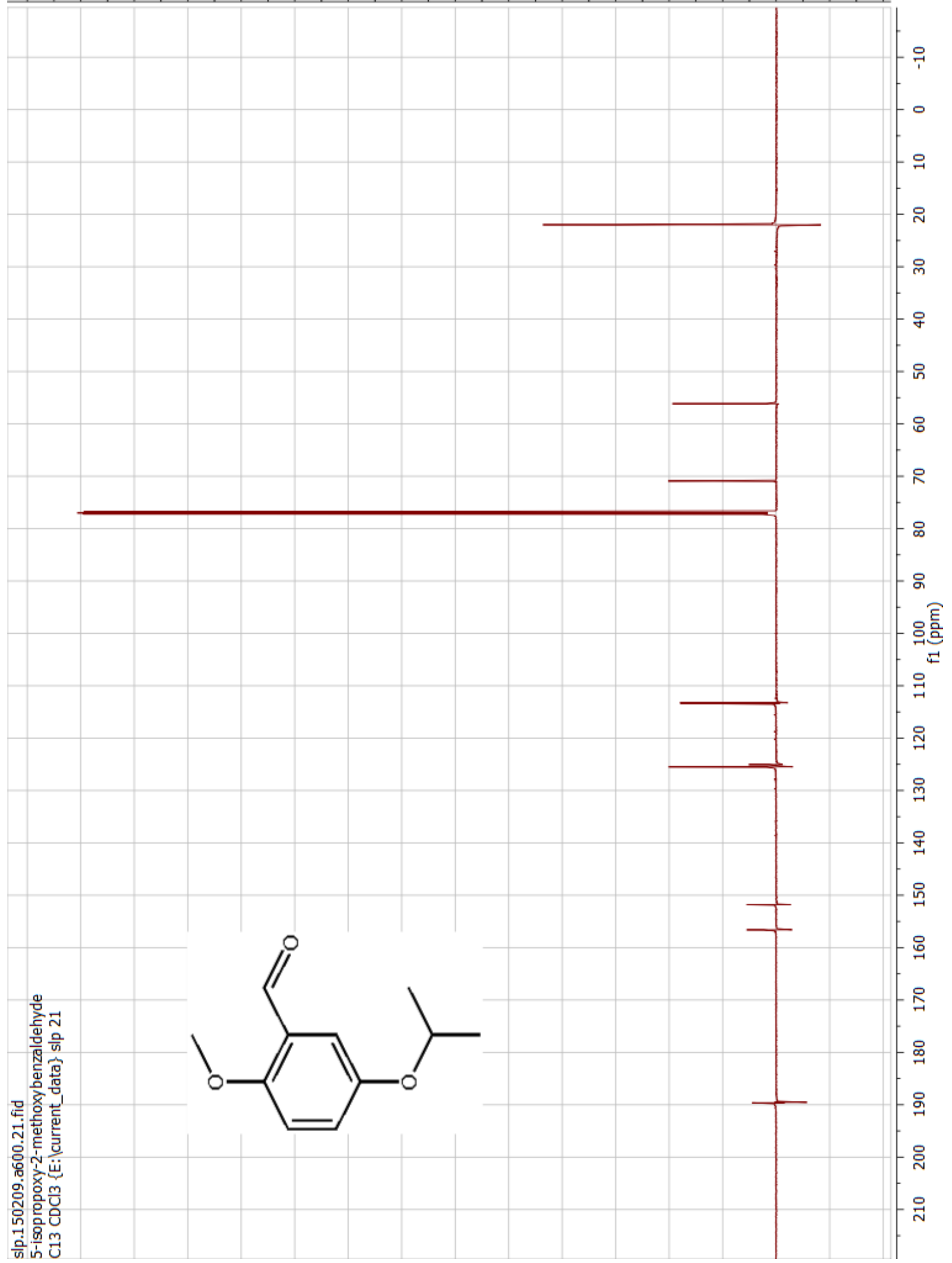




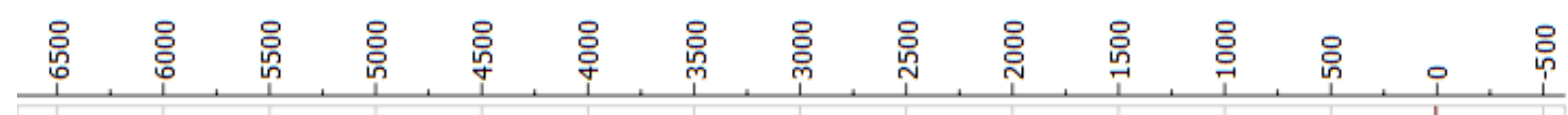

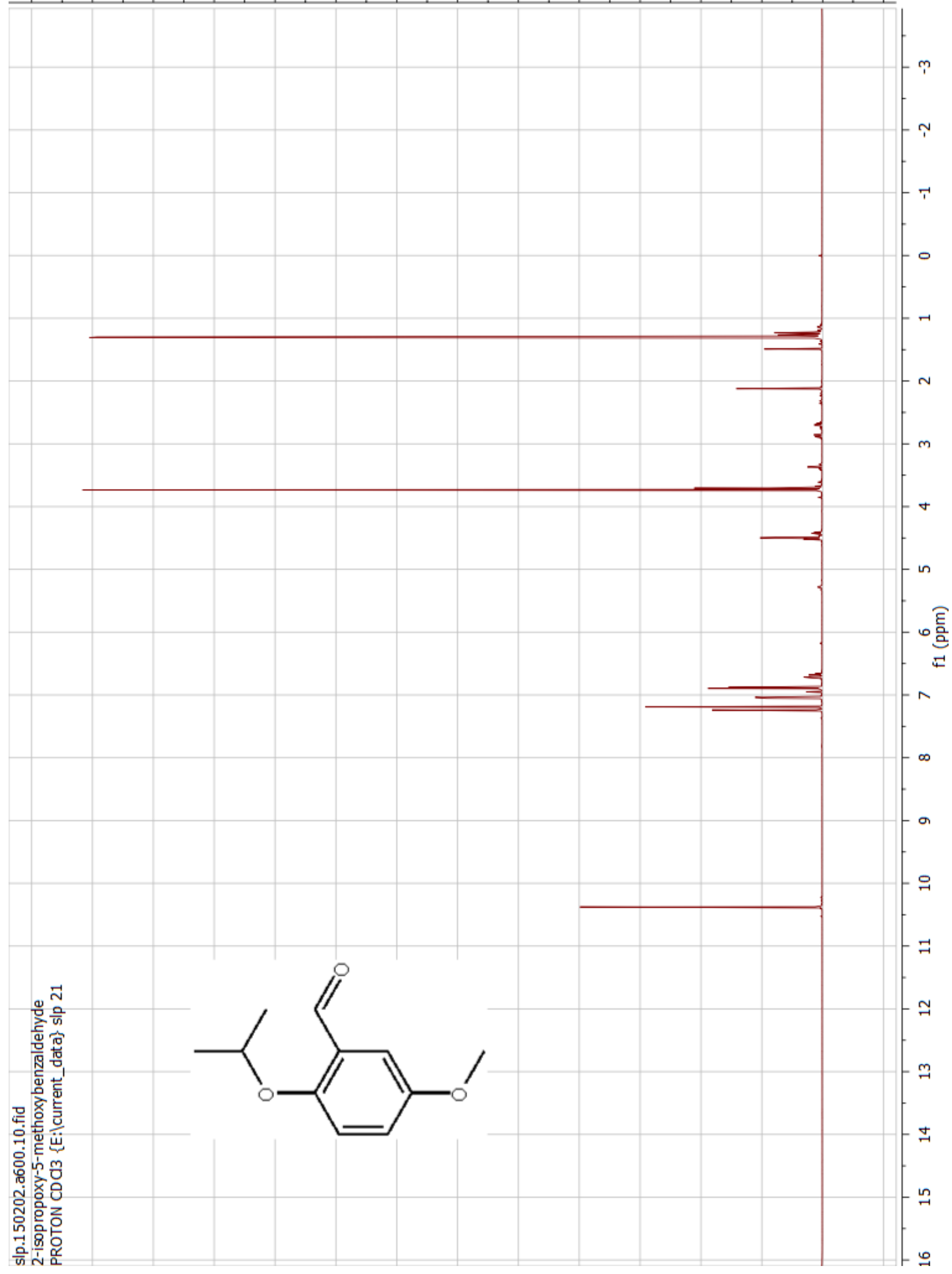




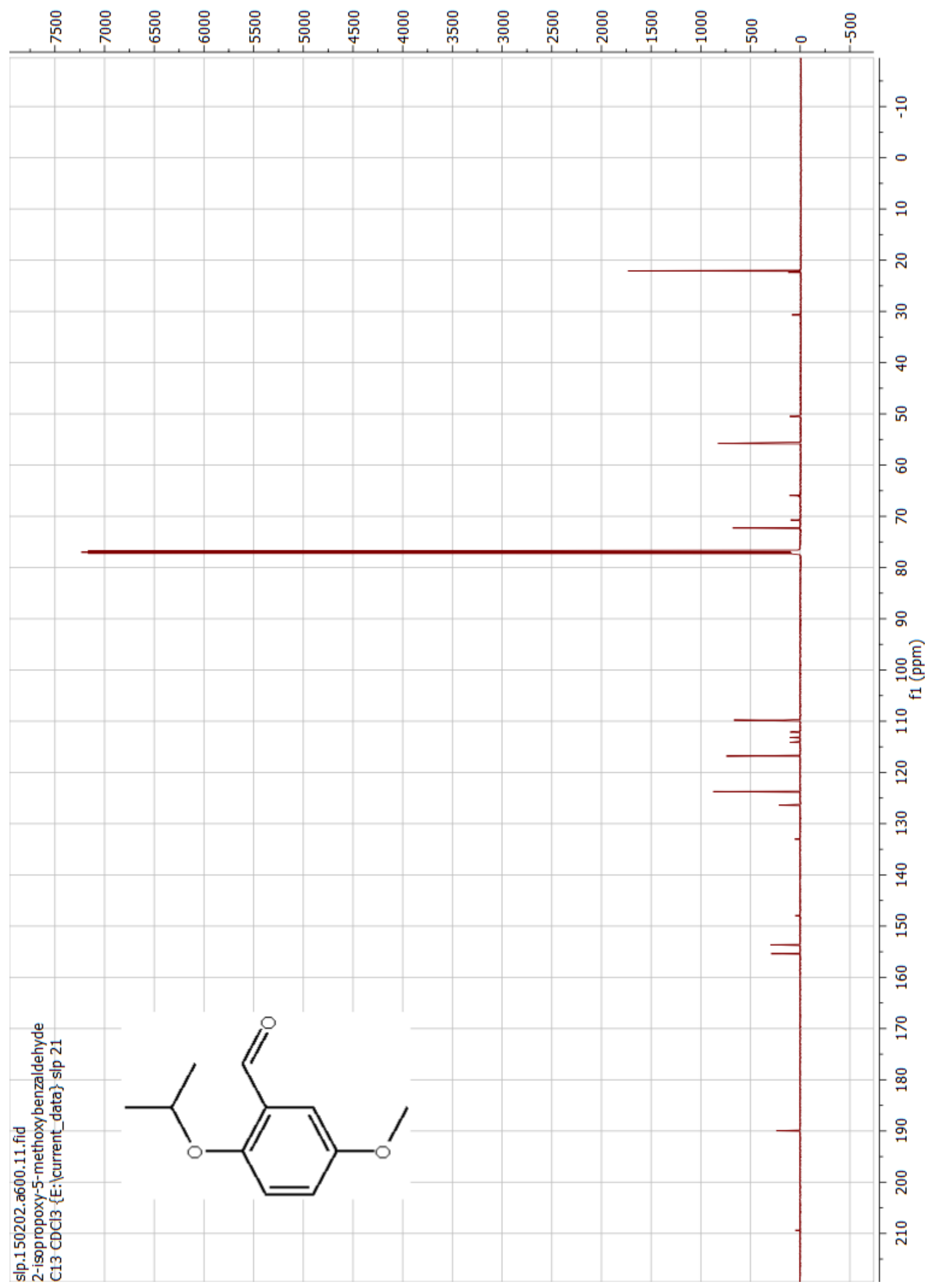




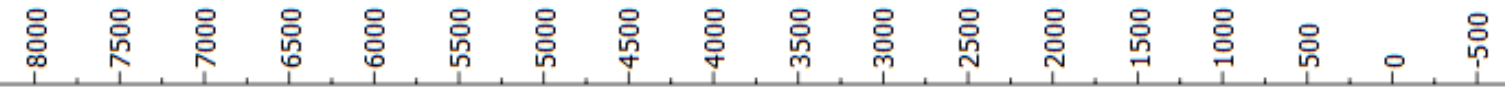

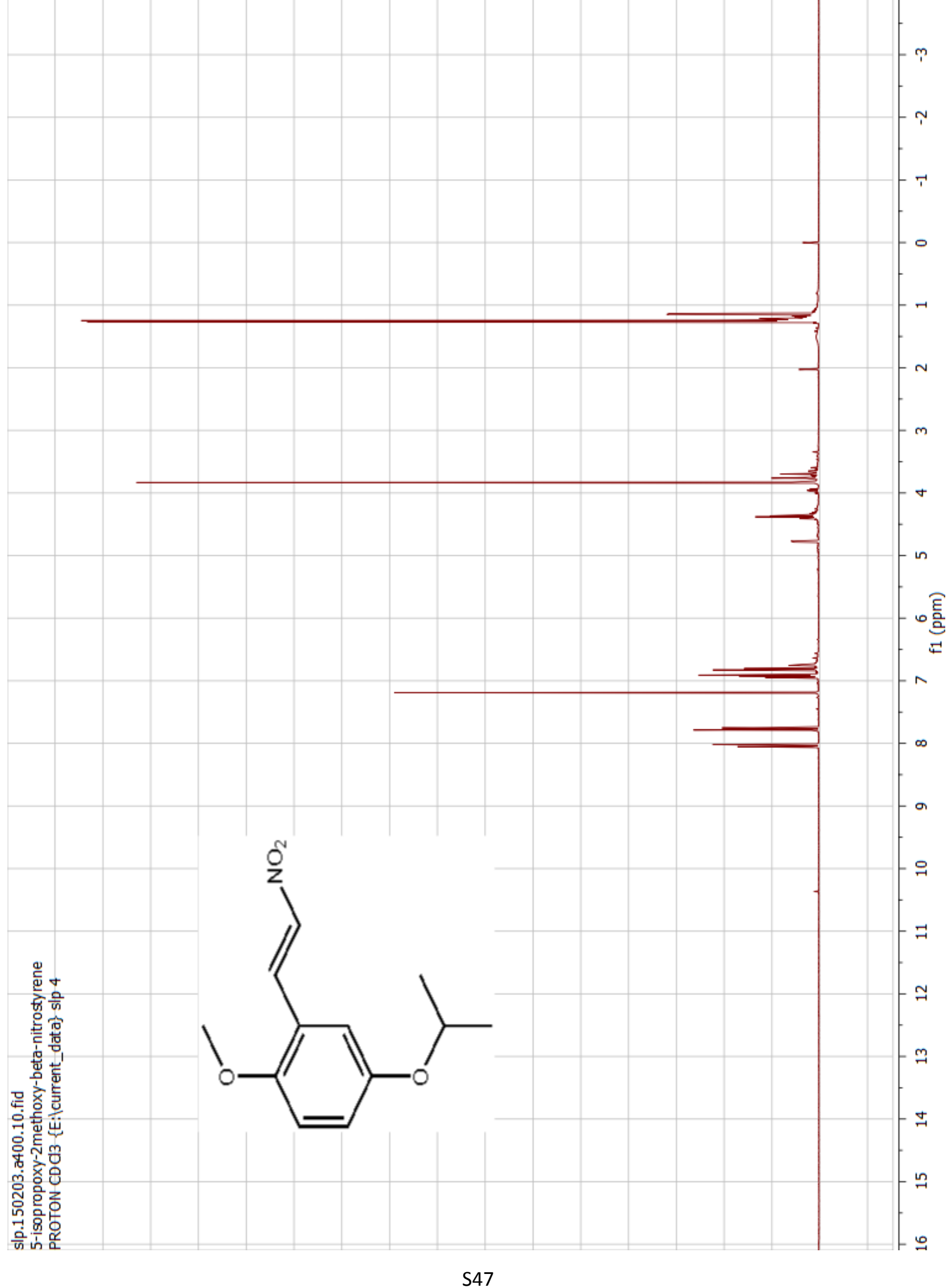




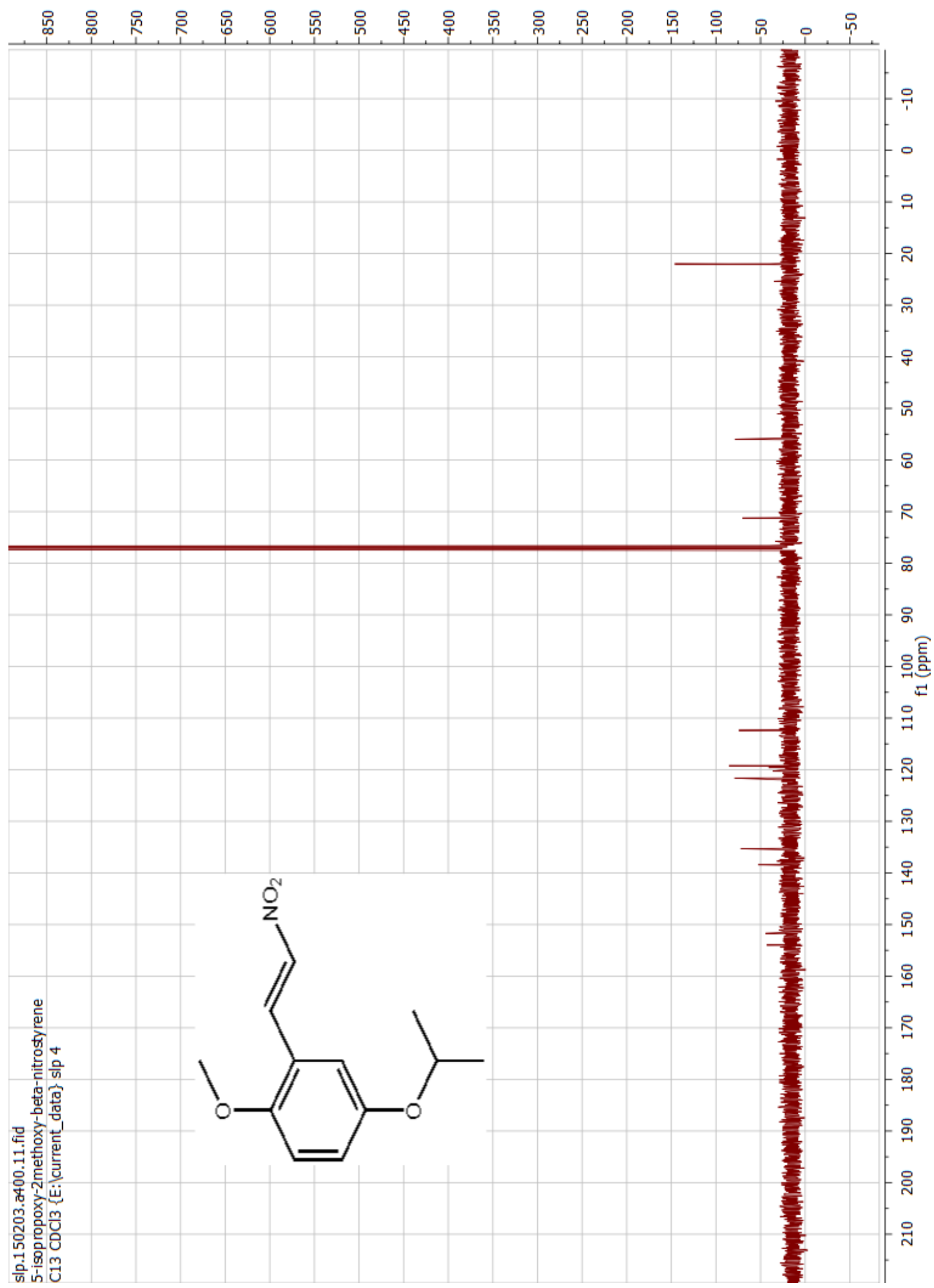




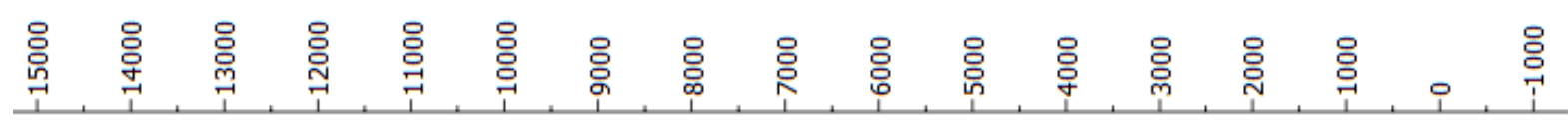

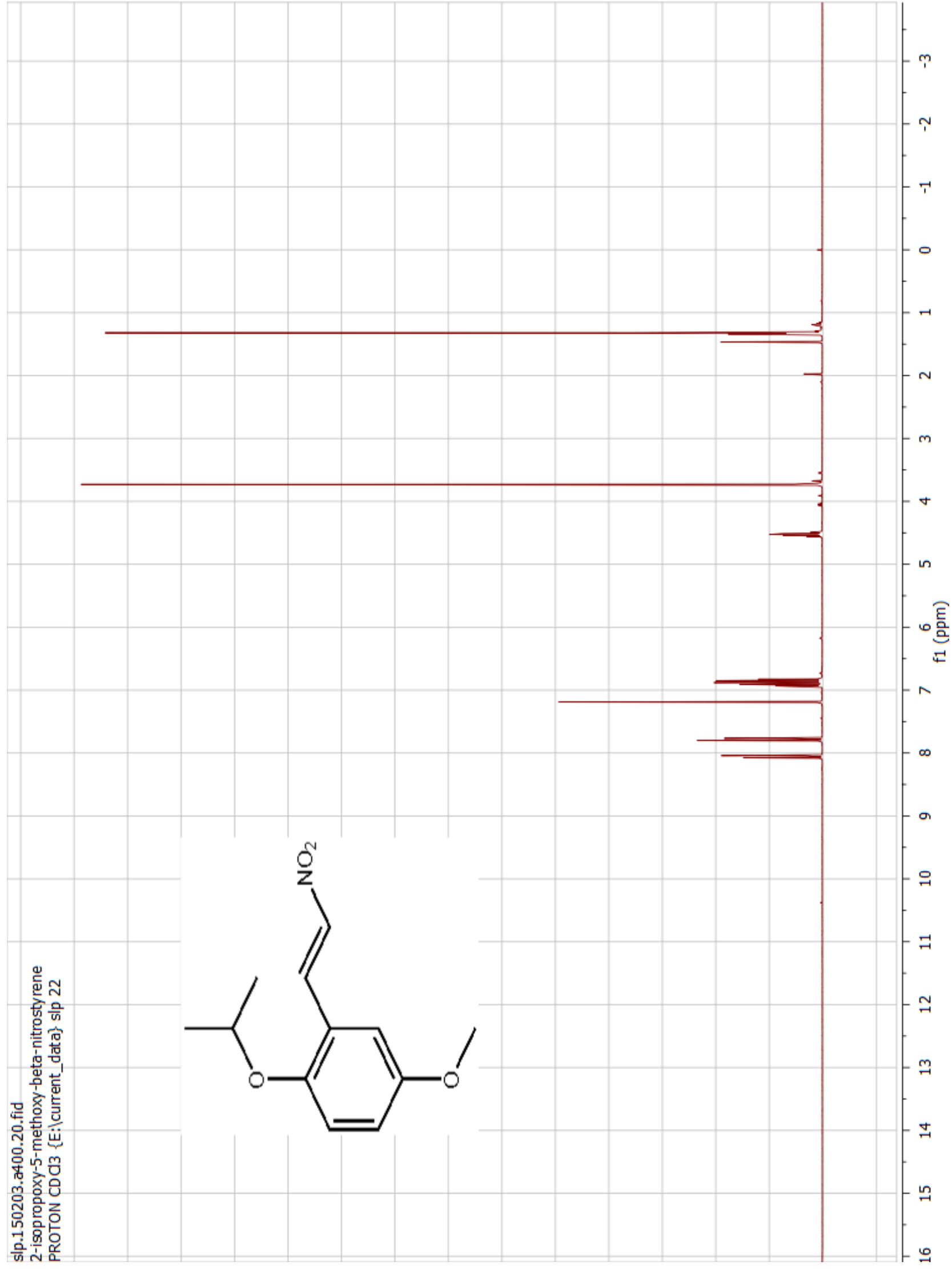




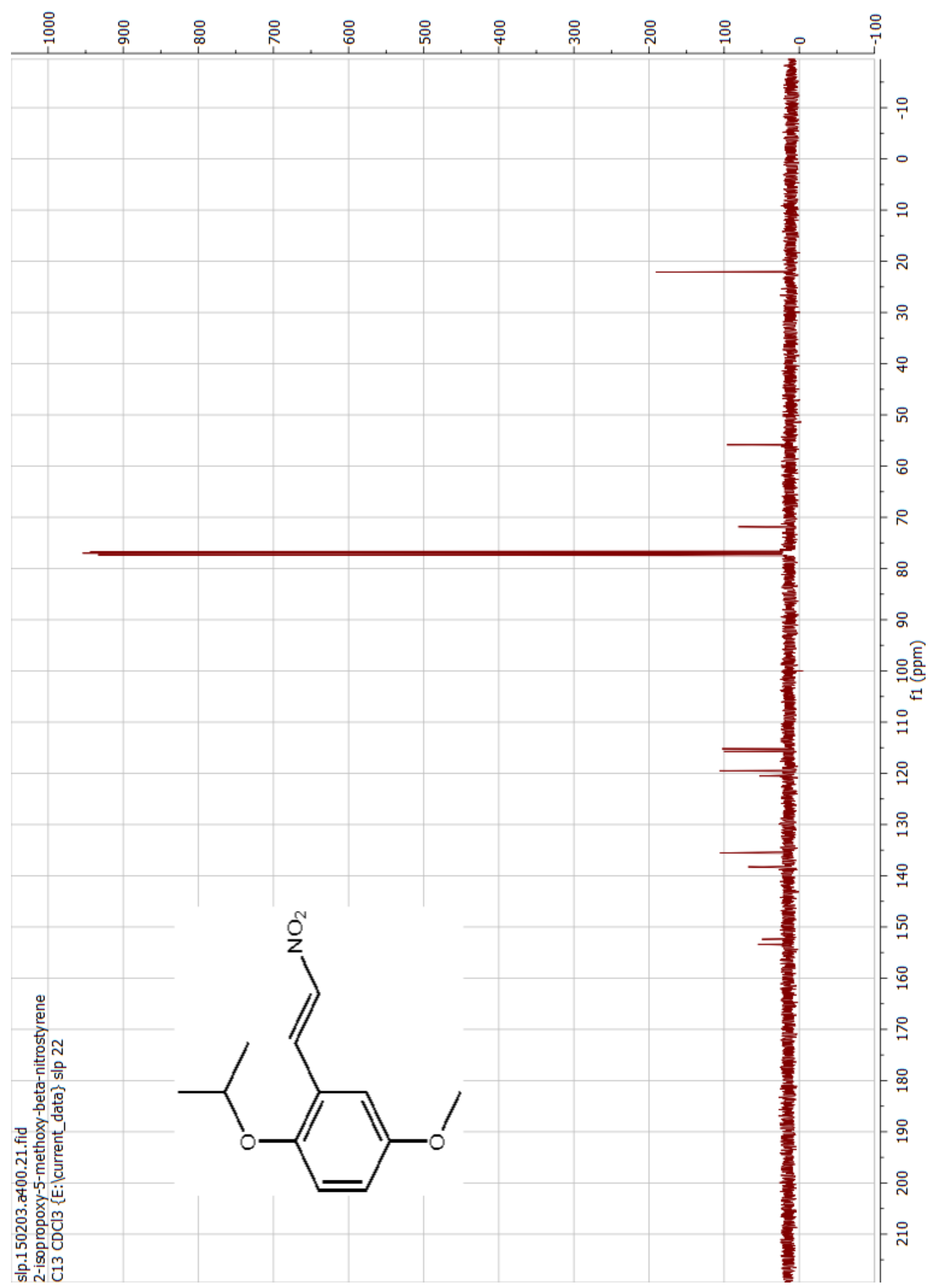




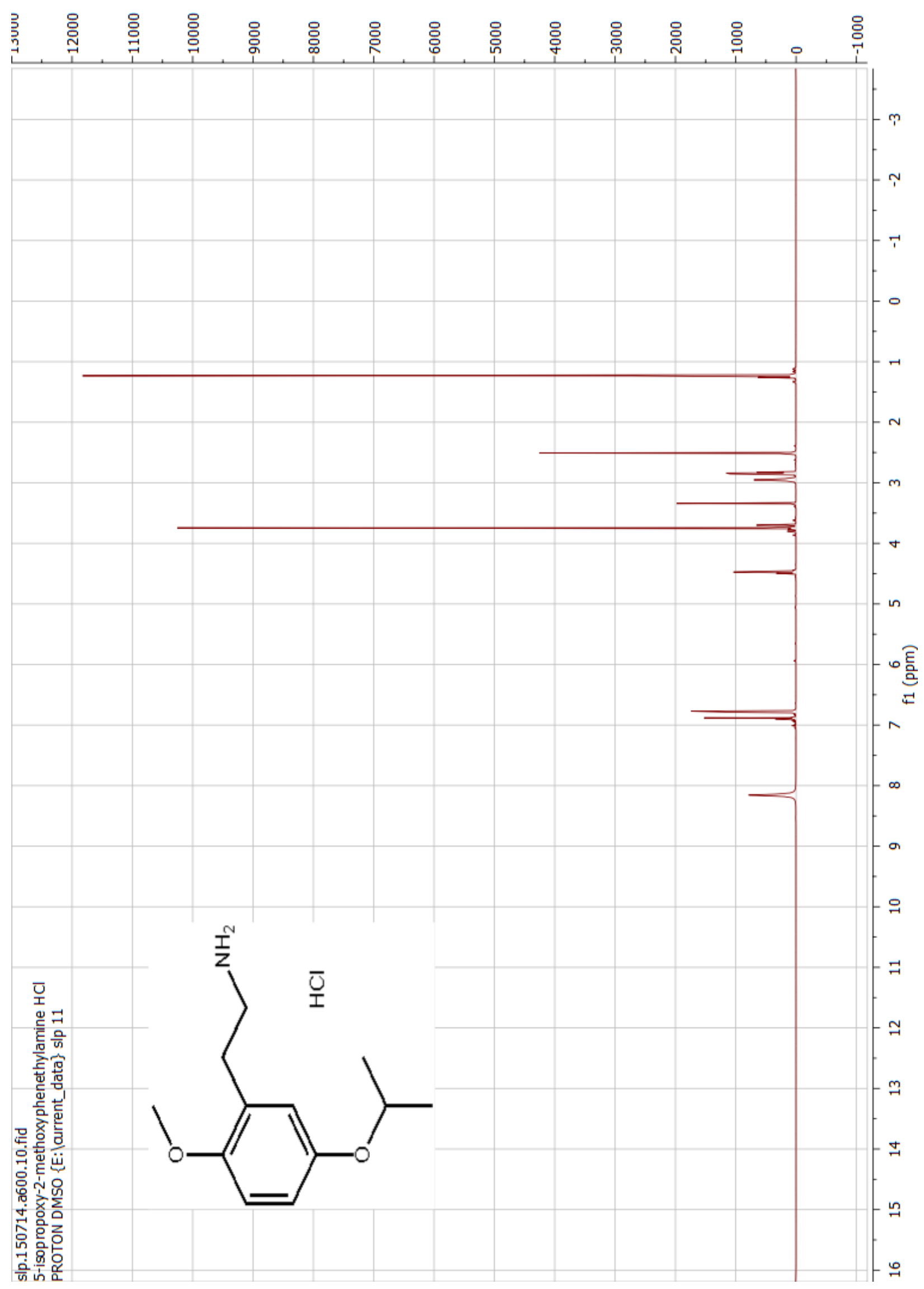




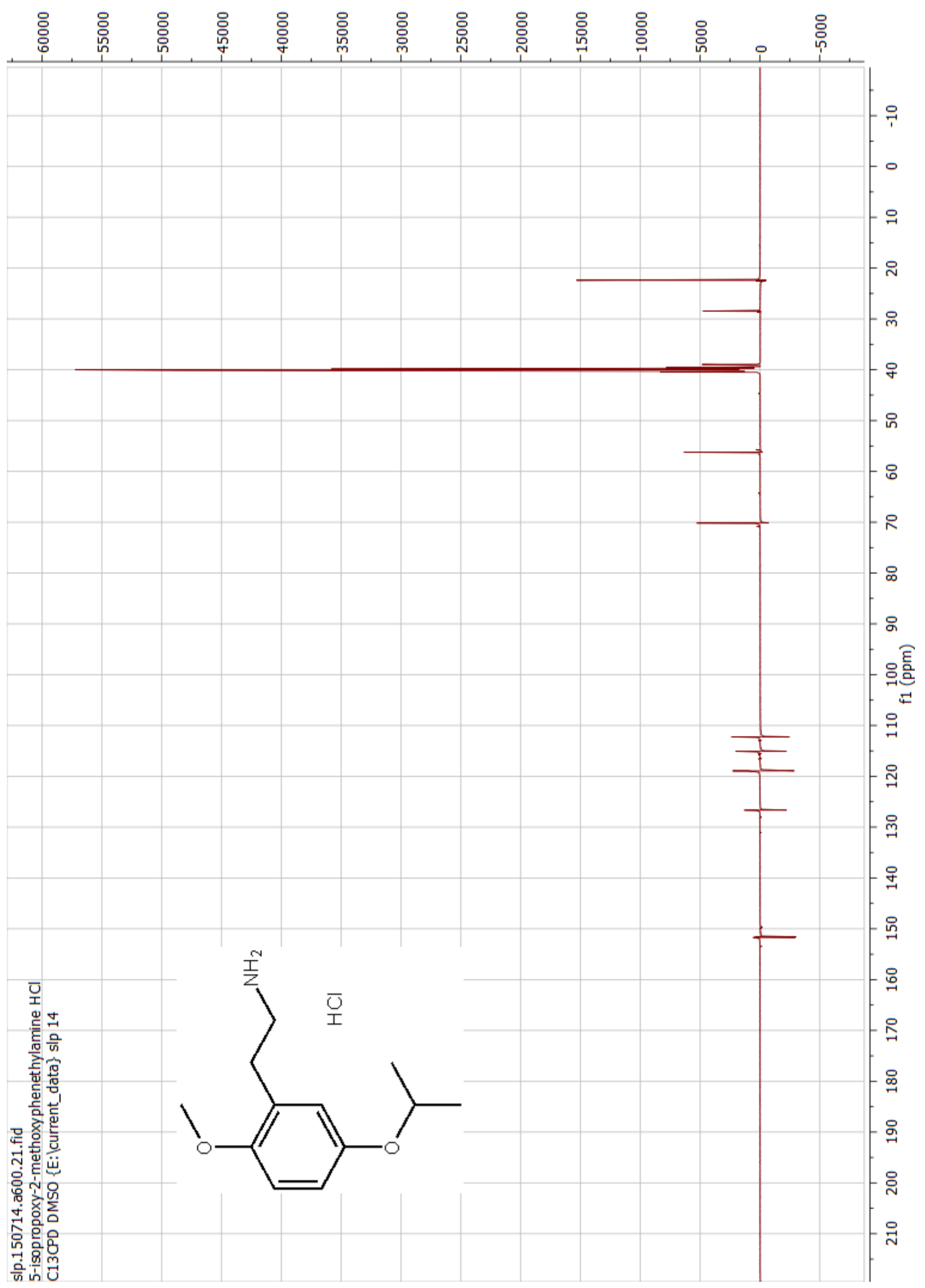




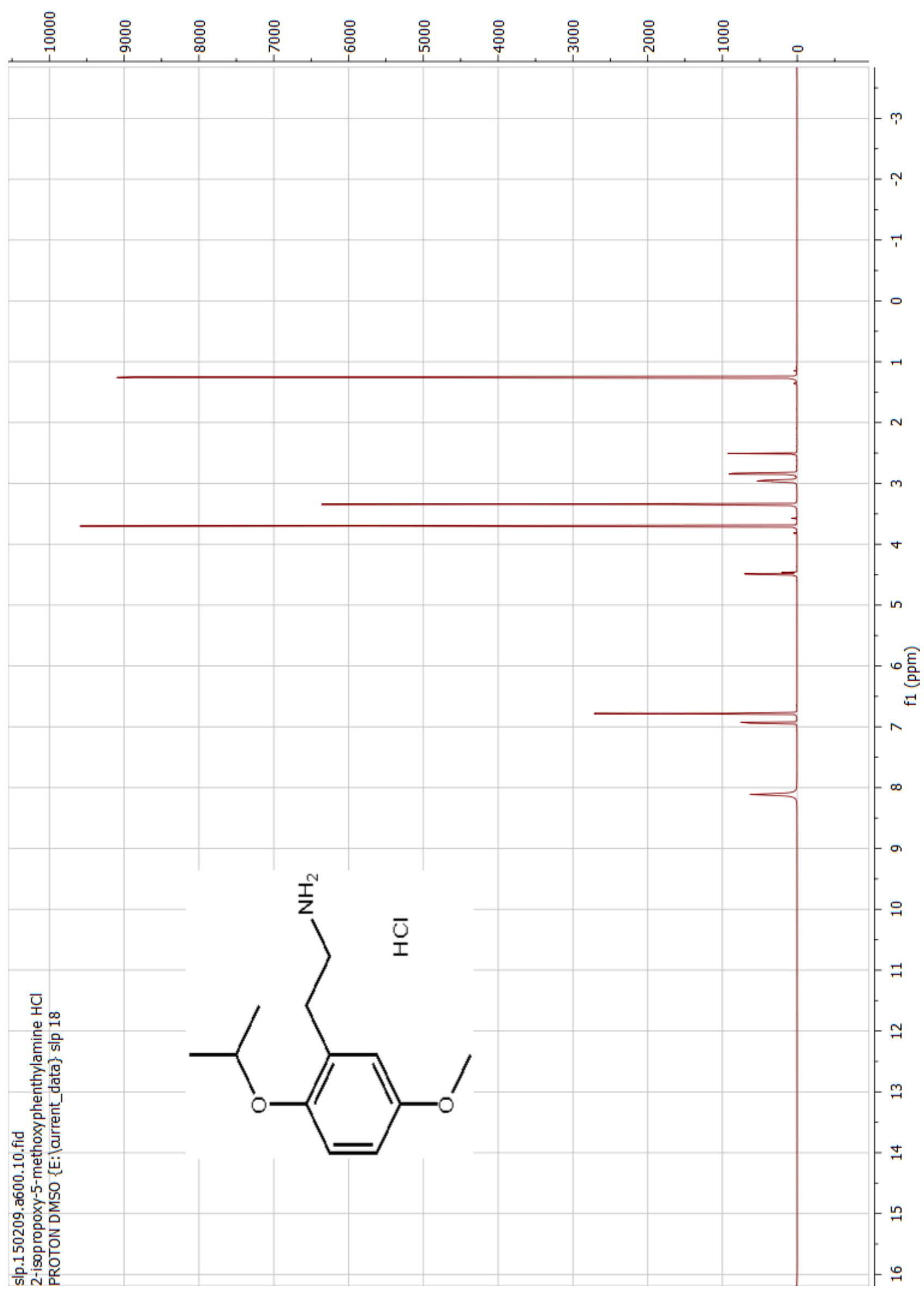




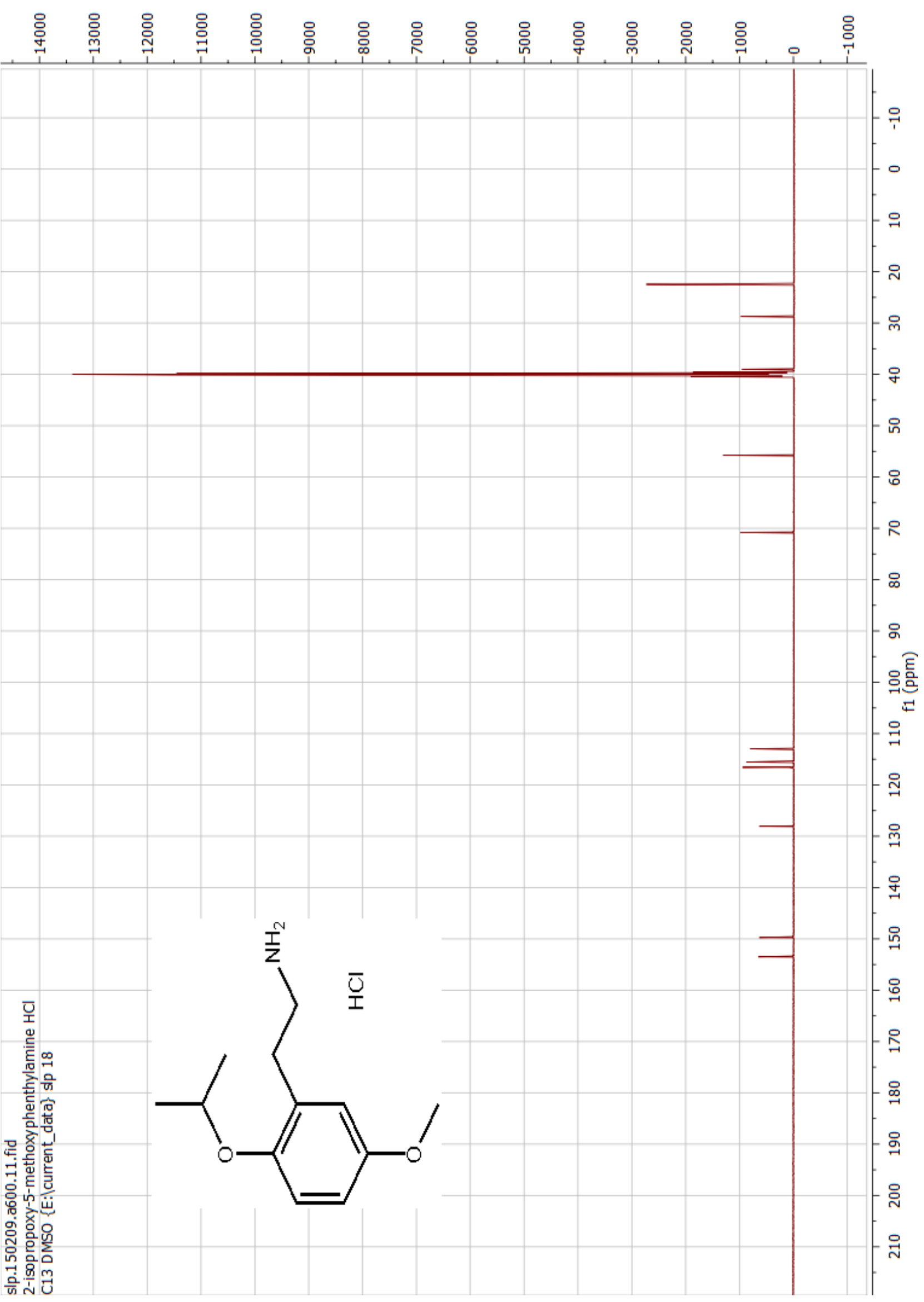




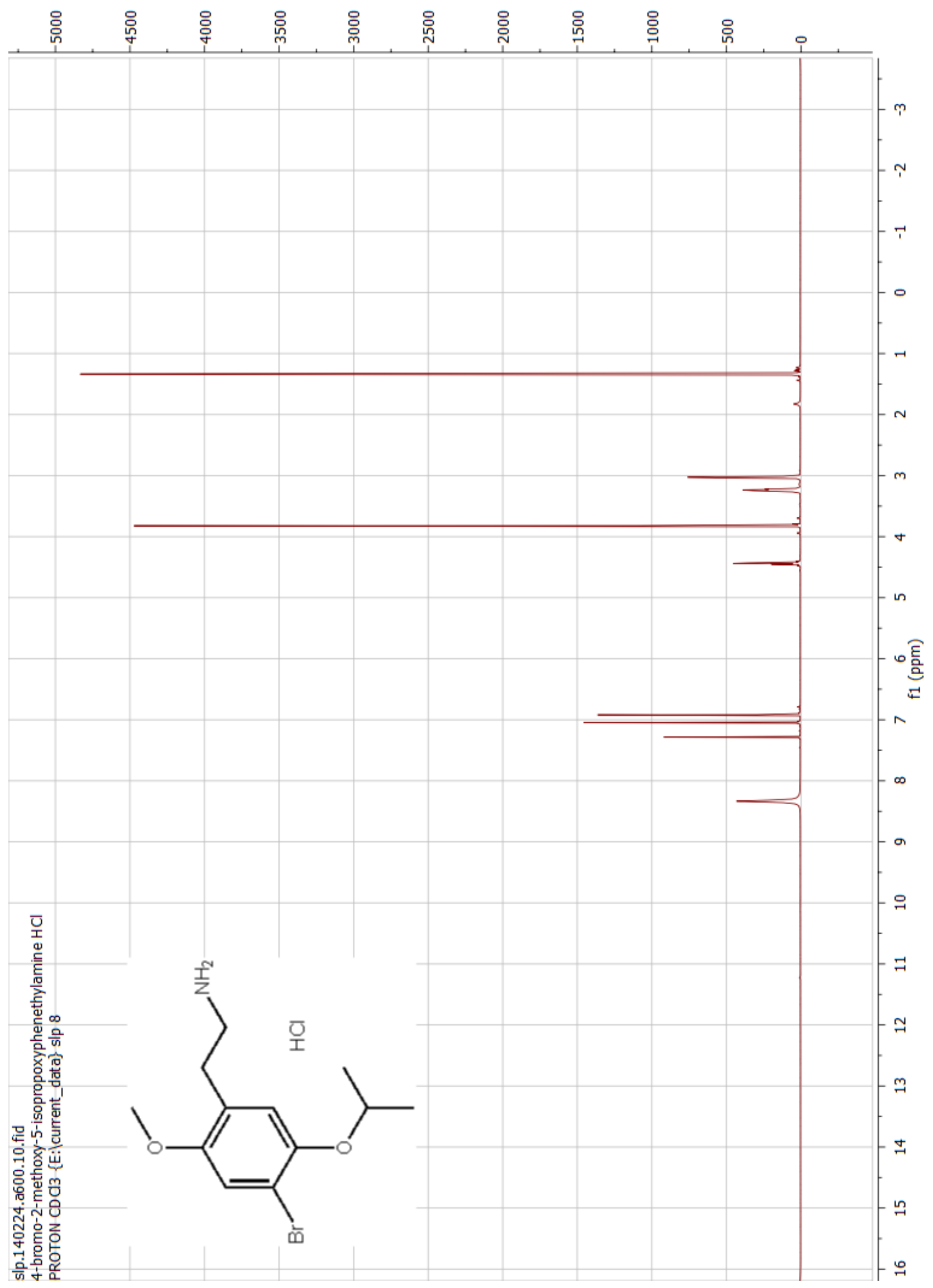




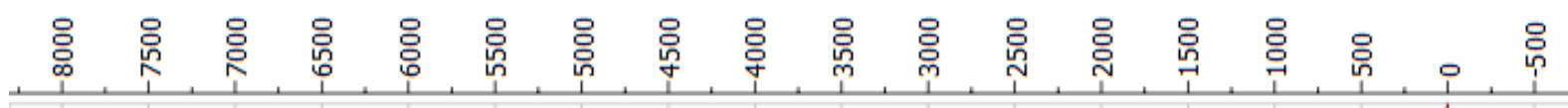

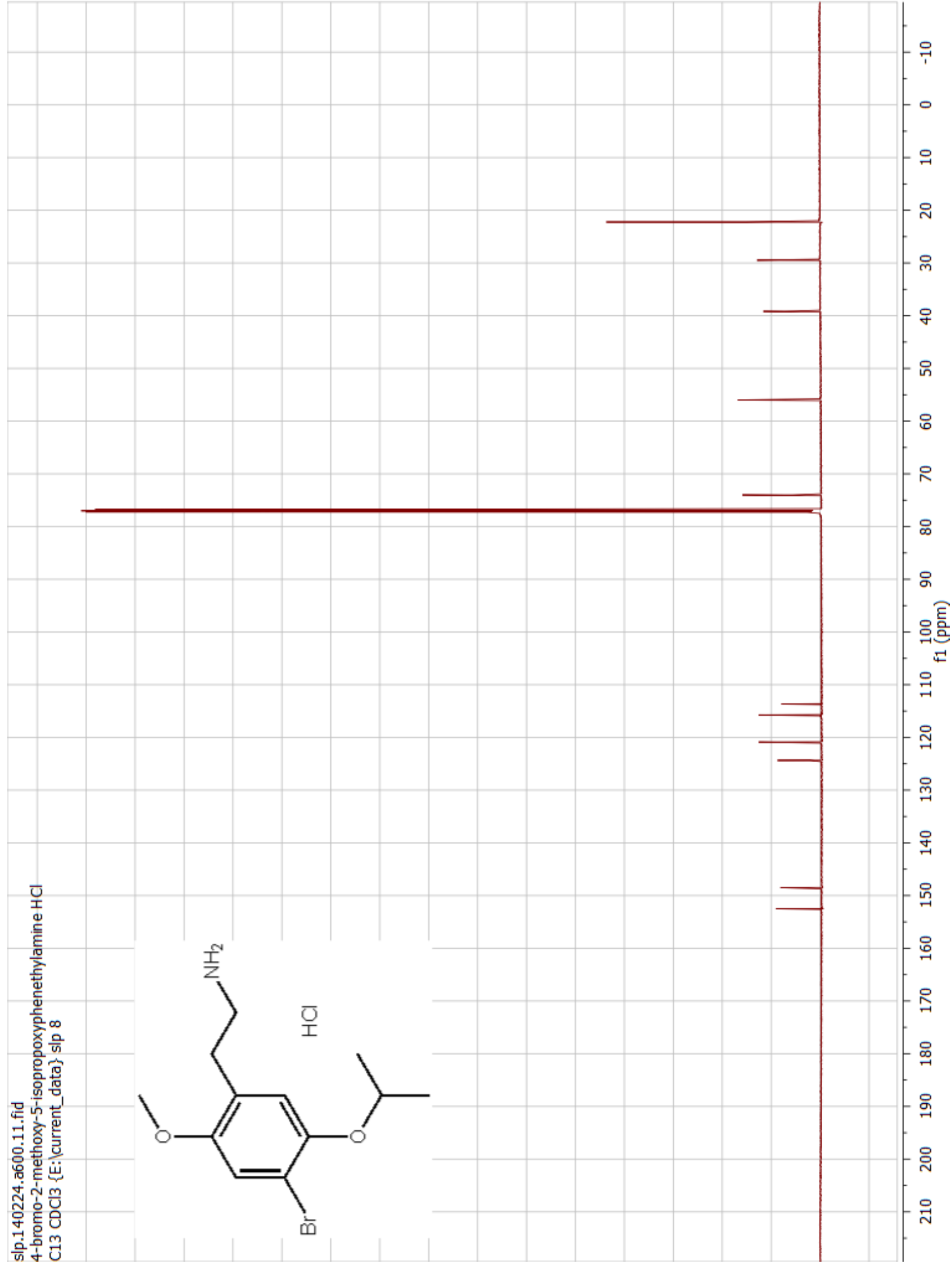




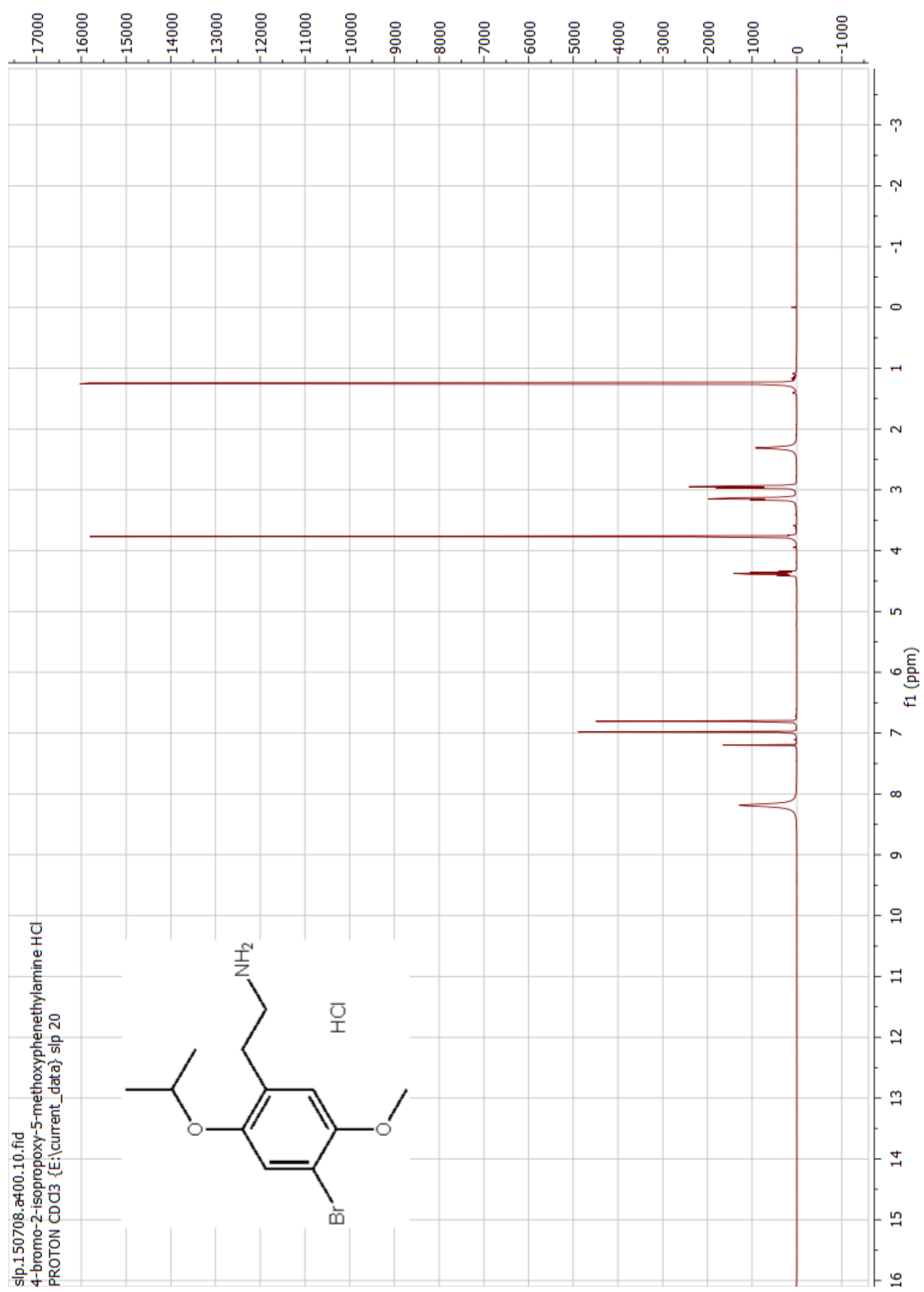




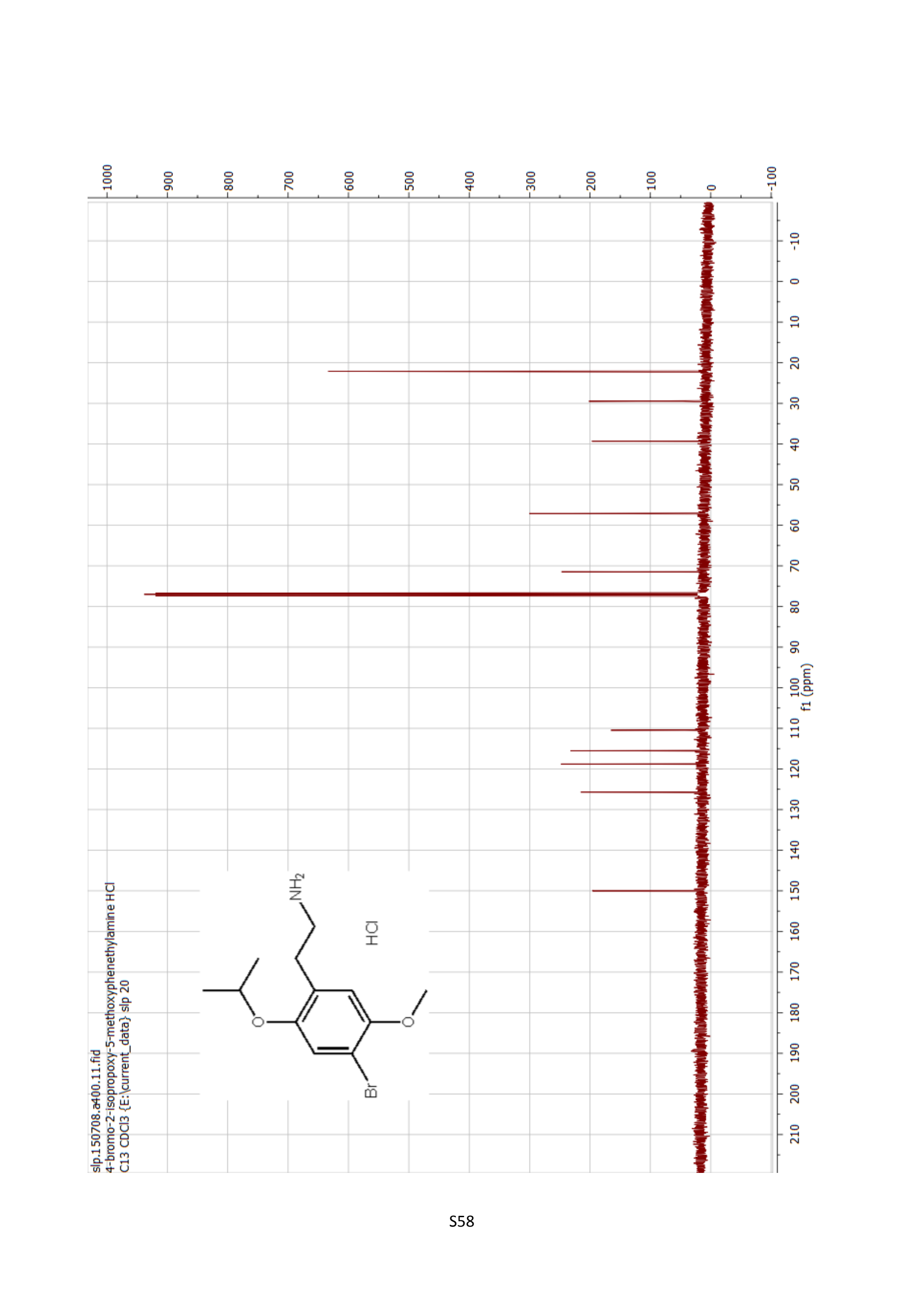




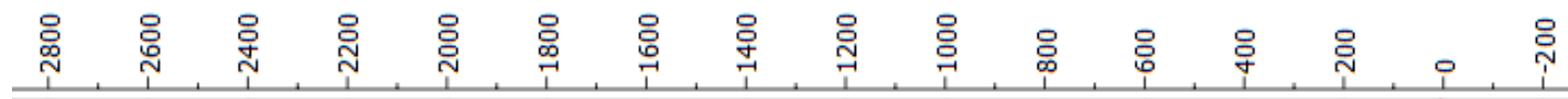

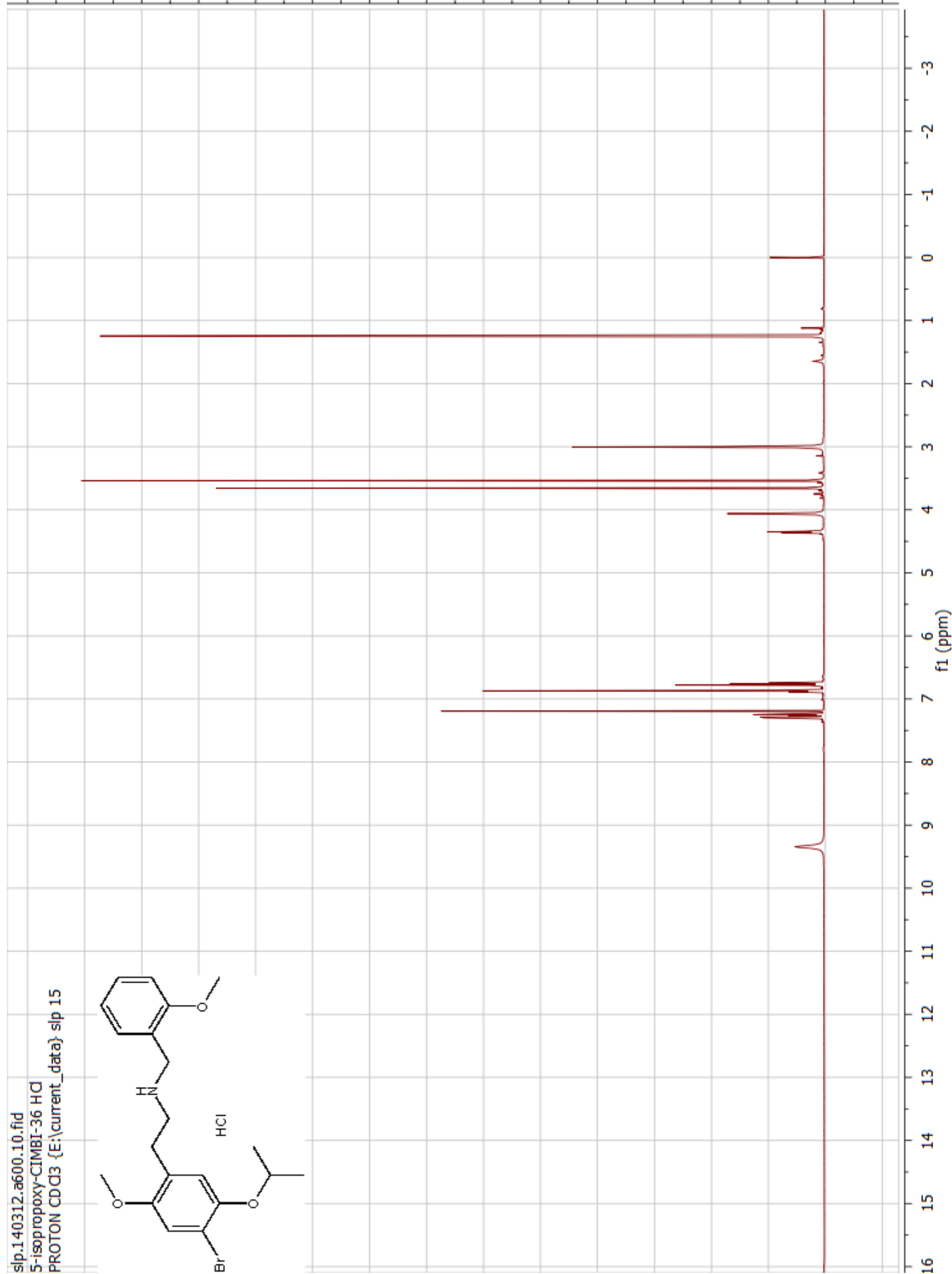




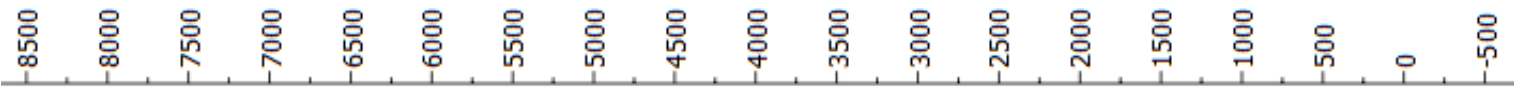

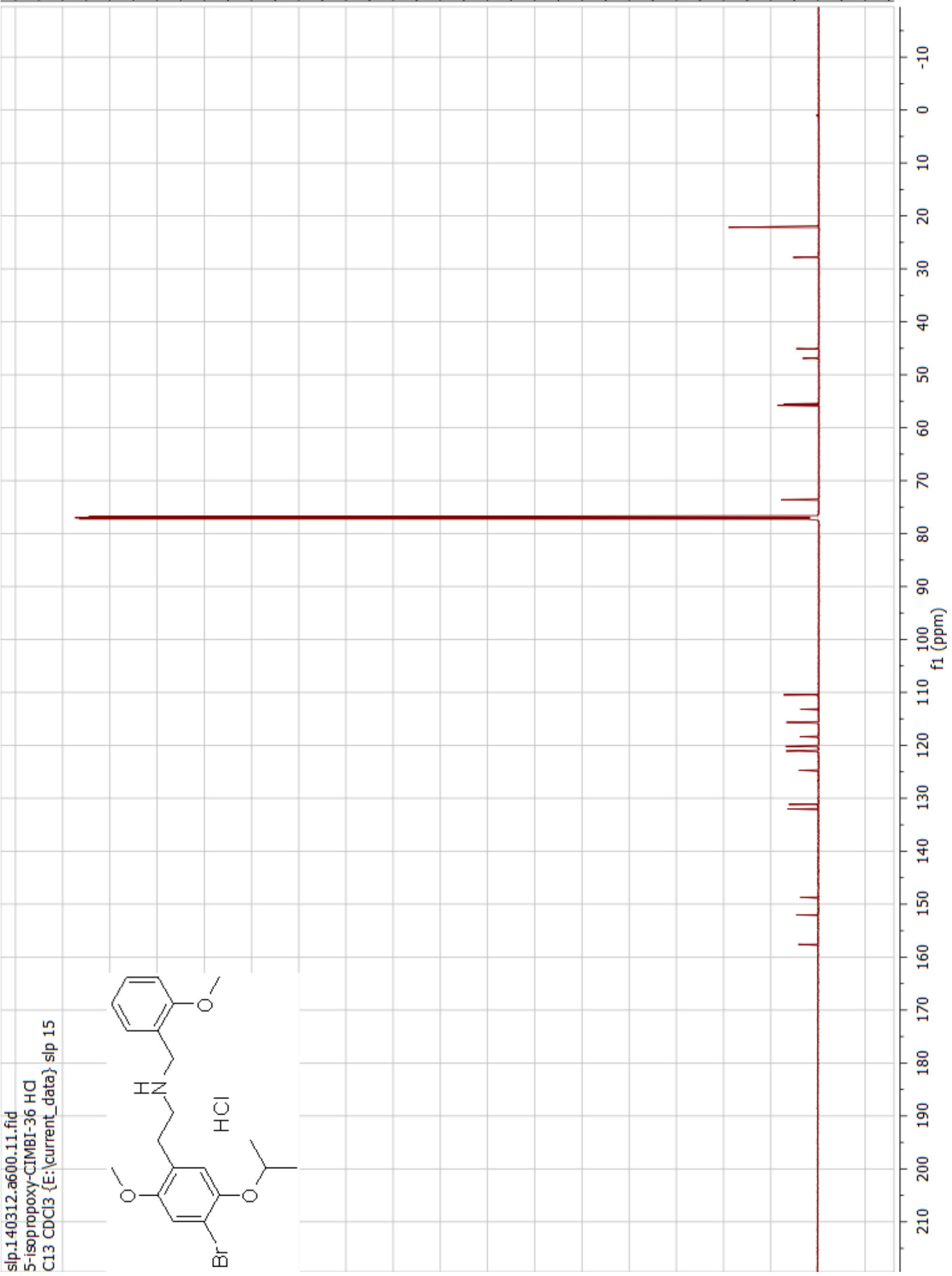




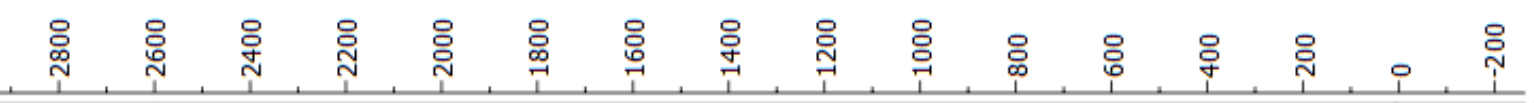

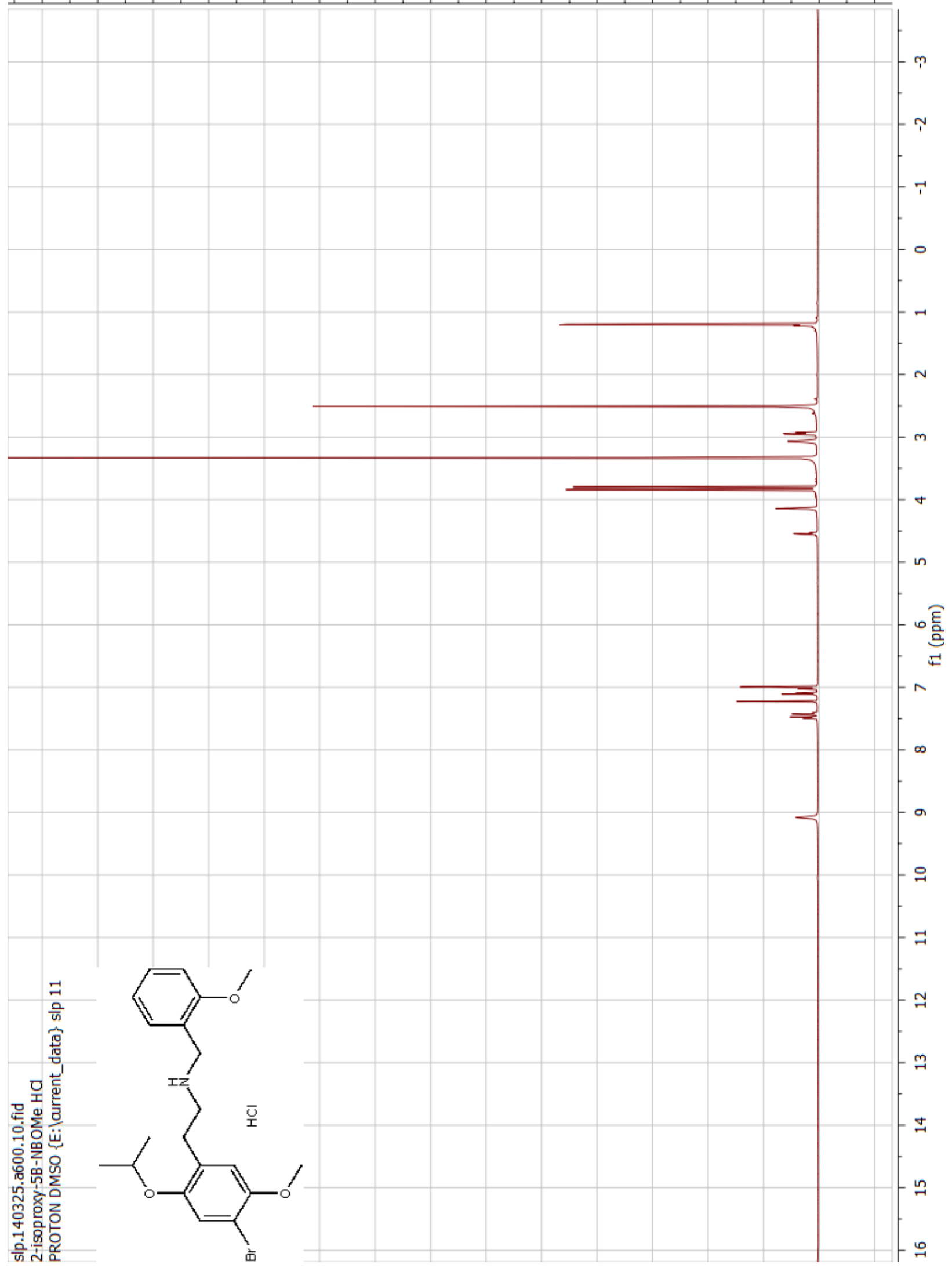




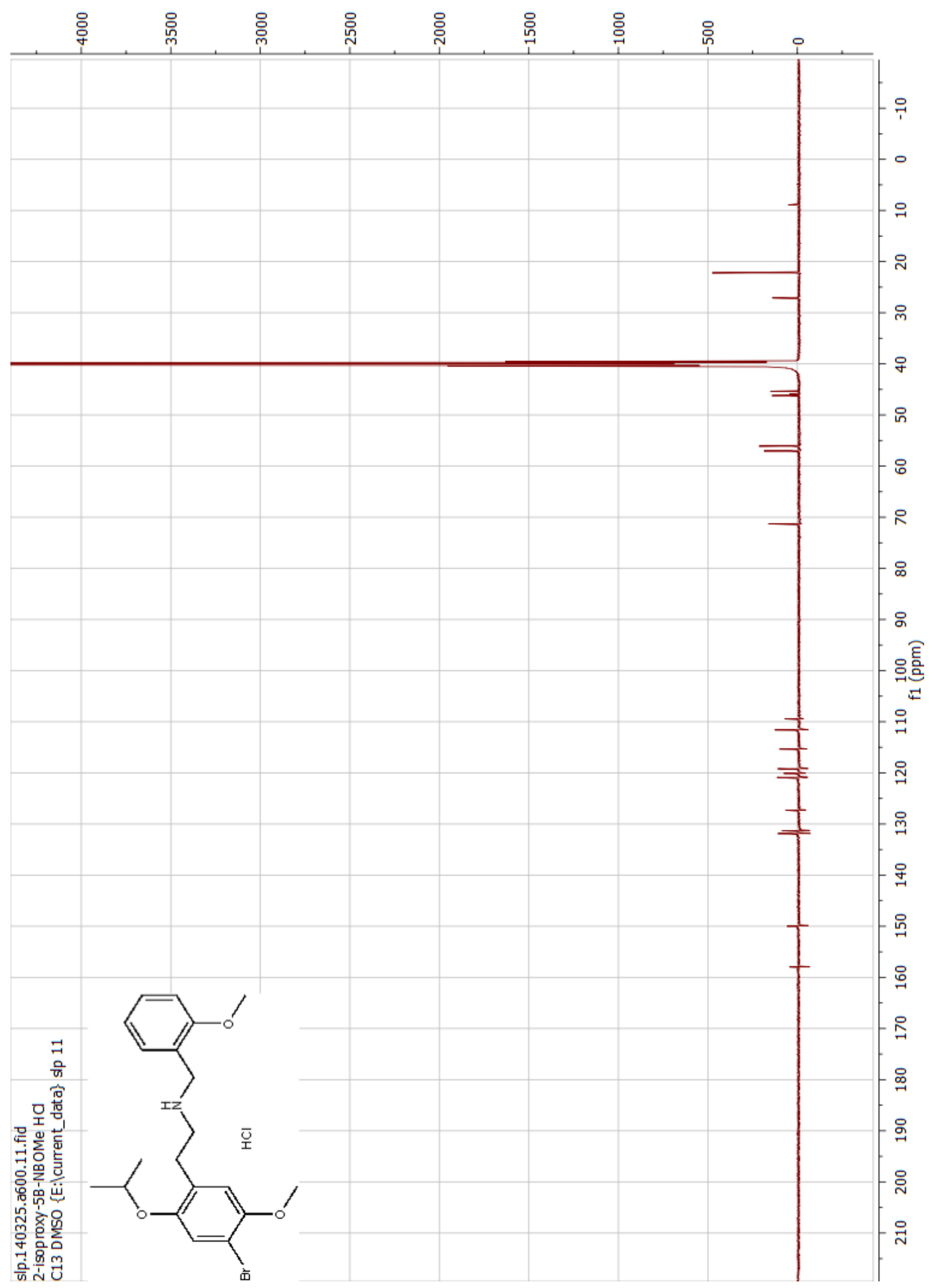




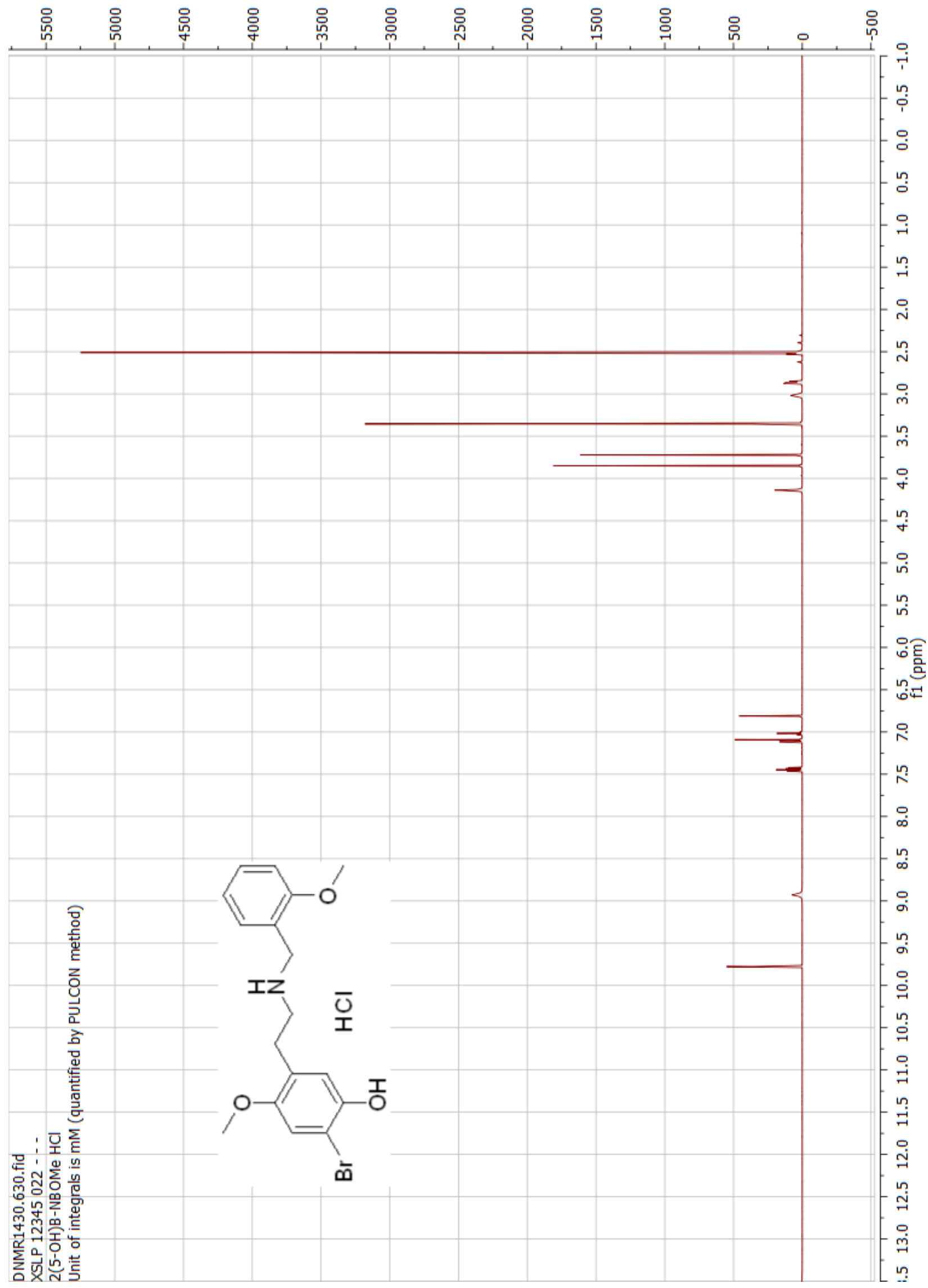




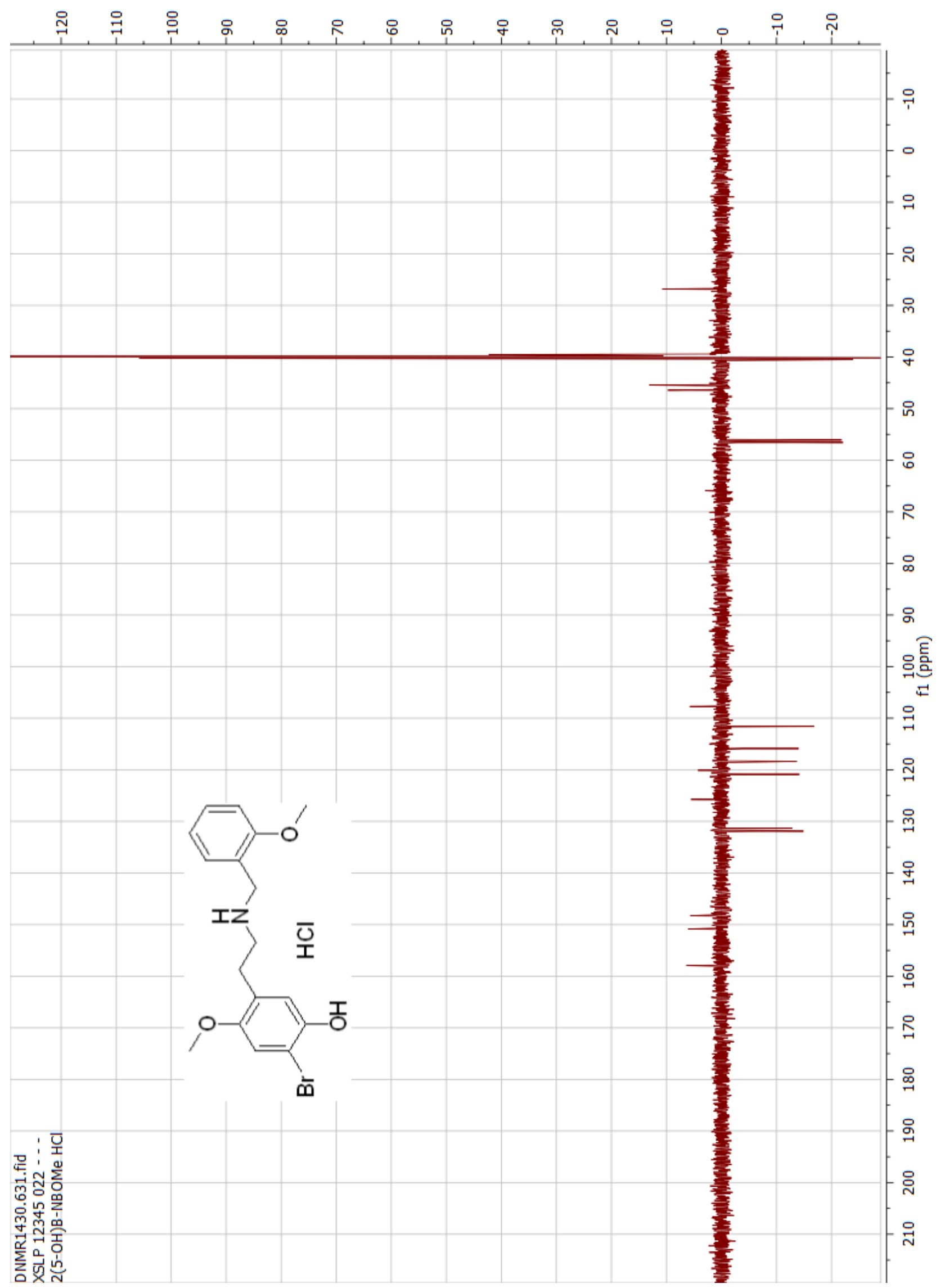


Openlynx Report - XSLP

Sample: 1

Vial: $1: 5$

Date:23-Jul-2014

ID-2 5-OH B-NBOMe-1

Inlet Method:Method452

Description:

Printed: Wed Jul 23 13:20:31 2014

\section{Sample Report:}

Sample 1 Vial 1:5 ID 2_5-OH_B-NBOMe-1 File 2_5-OH_B-NBOMe-1 Date 23-Jul-2014 Time 13:16:47 Description

1: MS APPI+ :TIC Smooth (SG, 2x2)

(2)

$5.9 e+008$

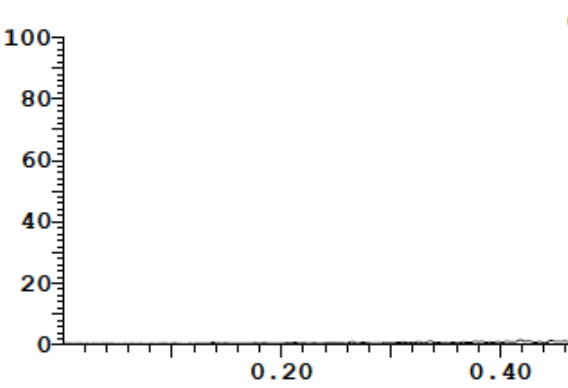

0.49

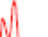

$\infty$

0.60

0.80

1.00

1.20

(2) PDA Ch1 254nme1.2nm

(2)

1008

0.50
8. $0 e^{-2}$
$6.0 e-2$
$4.0 e-2$
$2.0 e-2$
0.0

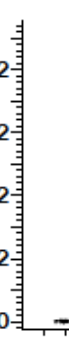

(3) ELSD Signal

(2)

1008

0.60

0.80

1.00

$9.527 e-2$

Range: $9.762 e-2$

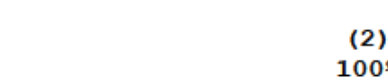

20

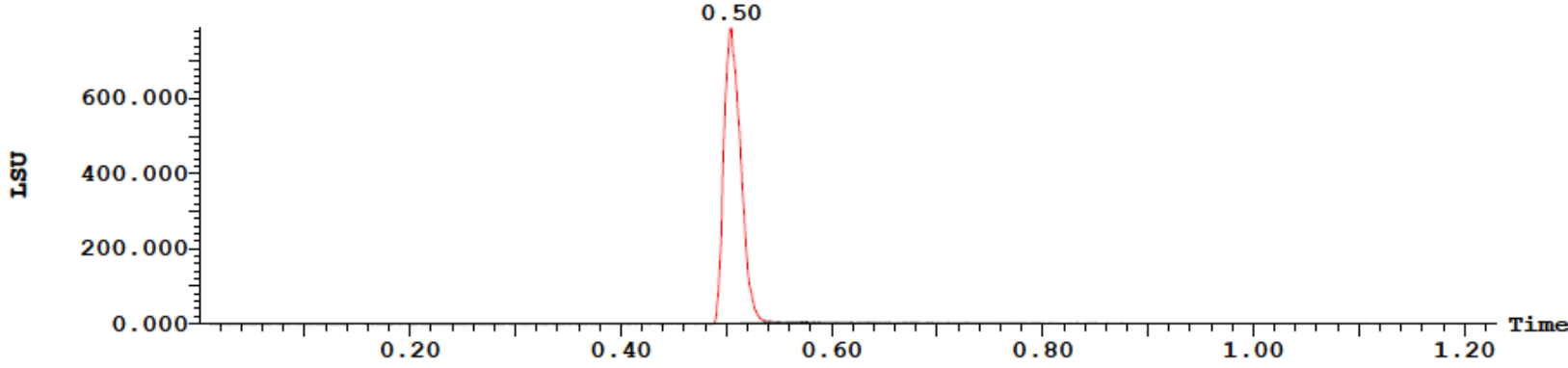


Openlynx Report - XSLP

Sample: 1

File:2_5-OH_B-NBOMe-1

Vial:1:5

Date:23-Jul-2014

ID:2_5-OH_B-NBOMe-1

Page 2

Description:

Inlet Method:Method452

Time: 13:16:47

Printed: Wed Jul 23 13:20:31 2014

Sample Report (continued):

Peak ID Compound Time Mass Found

$$
1
$$
0.48
Peak

2

${ }_{2}$ ID

Time
0.49

Mass Found

1:MS APPI+

$5.7 e+006$

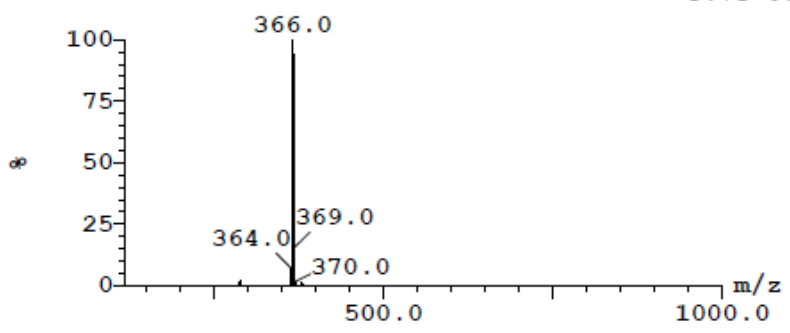

Peak ID Compound Time Mass Found

1:MS APPI+

2. $5 e+006$

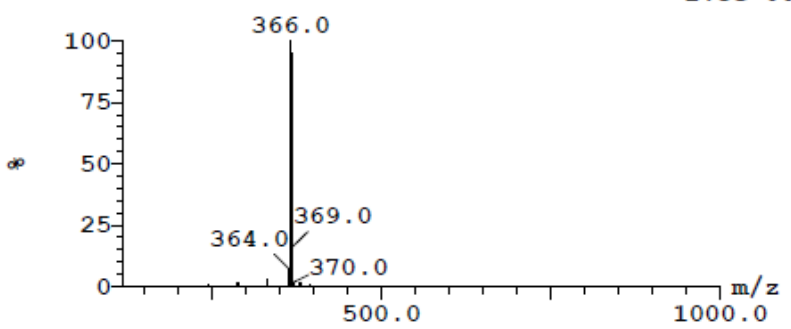

$1:$ MS APPI+

$5.8 e+006$

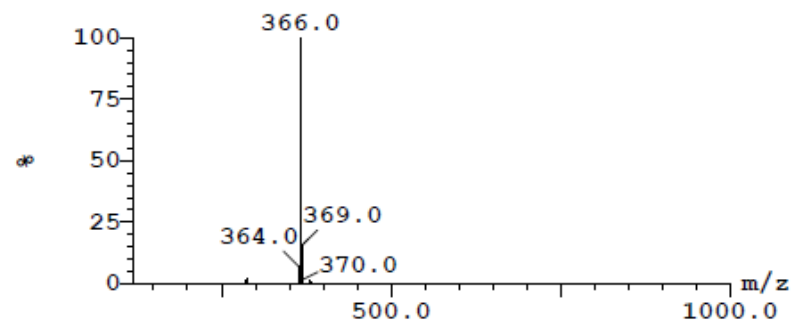




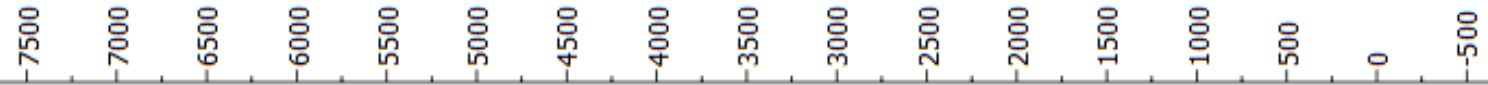

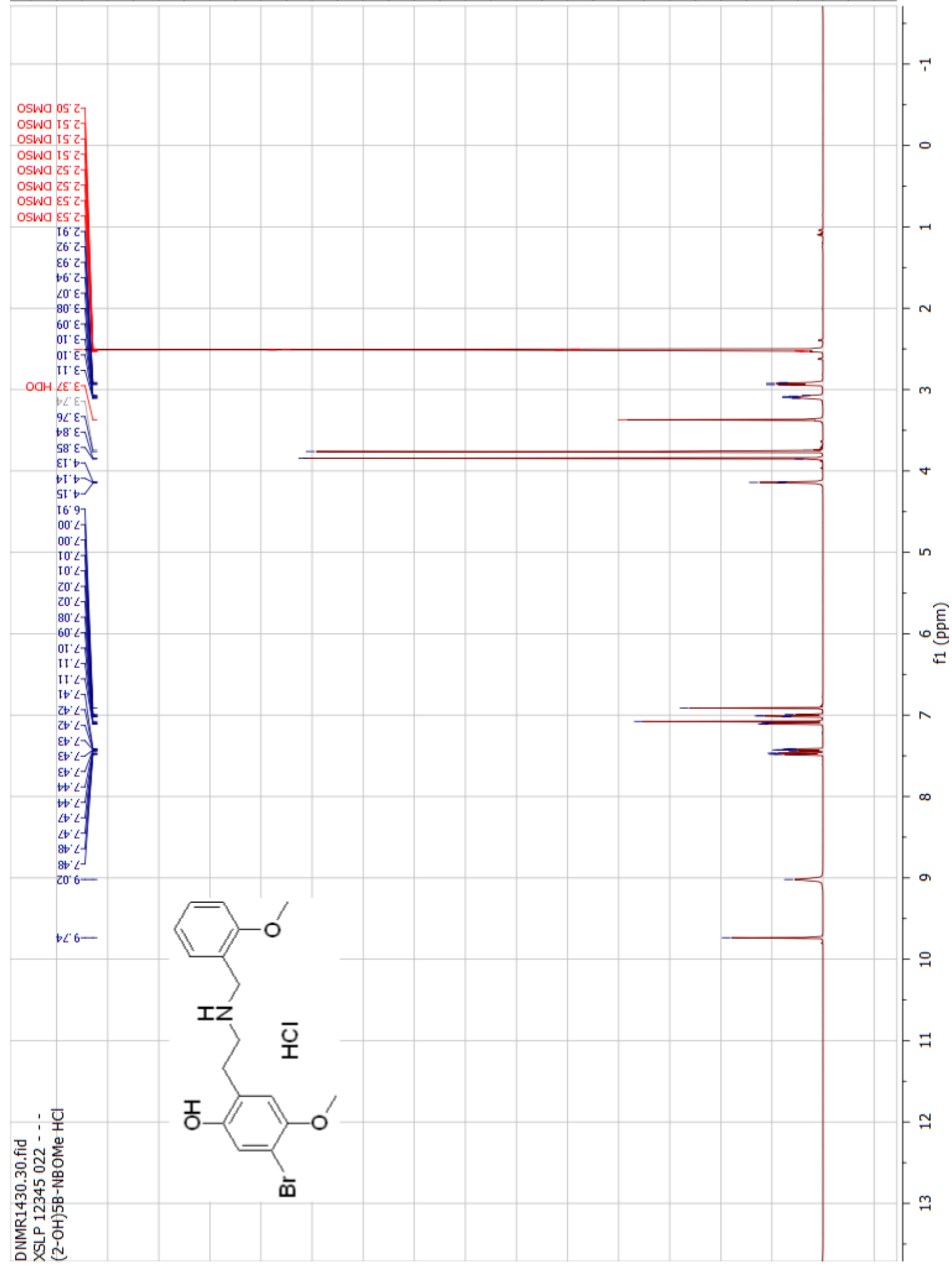




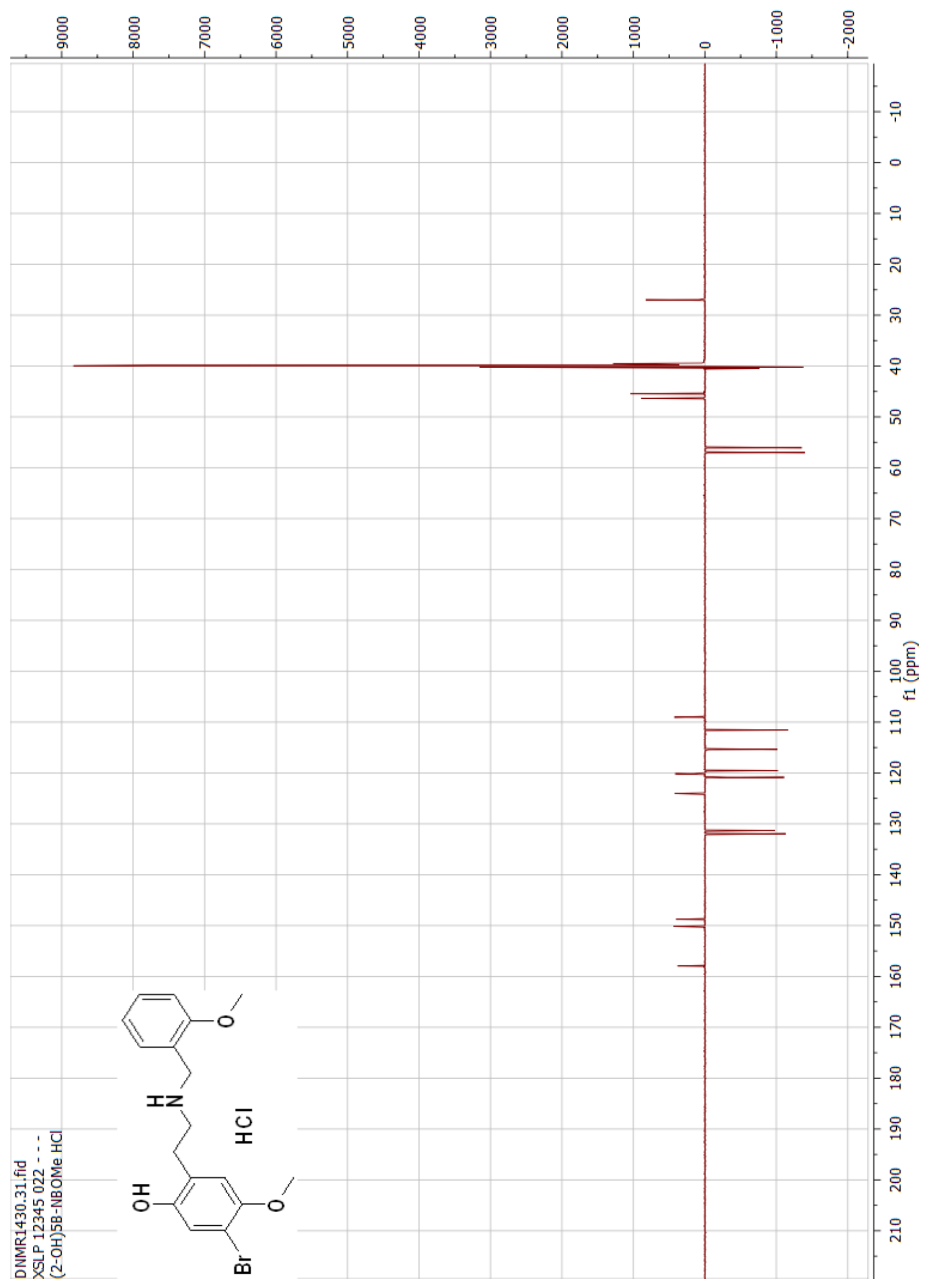


Sample Report:

Sample 1 Vial 1:26 ID 2-OH-5B-NBOMe File 2-OH-5B-NBOMe Date 21-Jul-2014 Time 10:24:35 Description

1: MS APPI+ :TIC Smooth (SG, $2 \times 2$ )

(2)

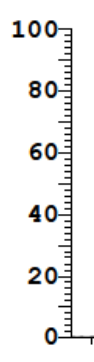
$(26)^{8}$ Q64) $(2) 0.468$ 468 - $48(2)$

$0.47\left[\begin{array}{r}46 \\ 0\end{array}\right.$

0.48

(2) PDA Ch1 $254 \mathrm{~nm} @ 1.2 \mathrm{~nm}$
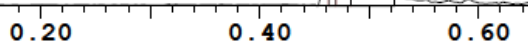

0.80

1.00

1.20

(2)

968

1. $277 e-1$

0.48

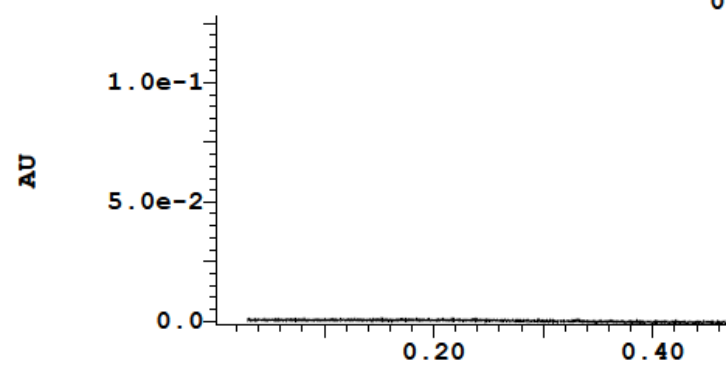

Range: $1.293 \mathrm{e}-1$

(3) ELSD Signal
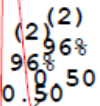

0.5050

0.40

0.60

0.80

1.20

(3)

998

250.000責

0.49

Range: 1222.121 
Printed: Mon Jul 21 10:30:20 2014

Sample Report (continued):

Peak ID Compound Time Mass Found $1 \quad 0.47$$$
369 .
$$

364.0

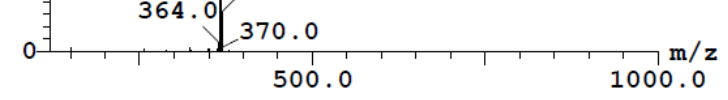

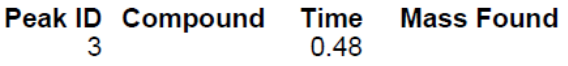

\section{1:MS APPI+}

8. $0 e+006$

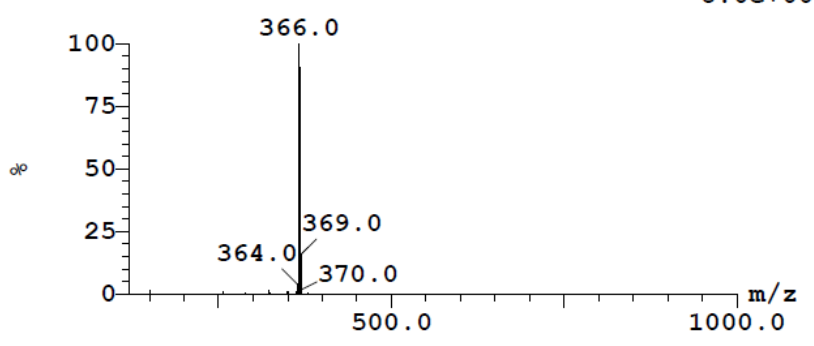

Peak ID Compound Time Mass Found

$1:$ MS APPI+

7. $9 e+006$

$1:$ MS APPI+

8. $0 e+006$

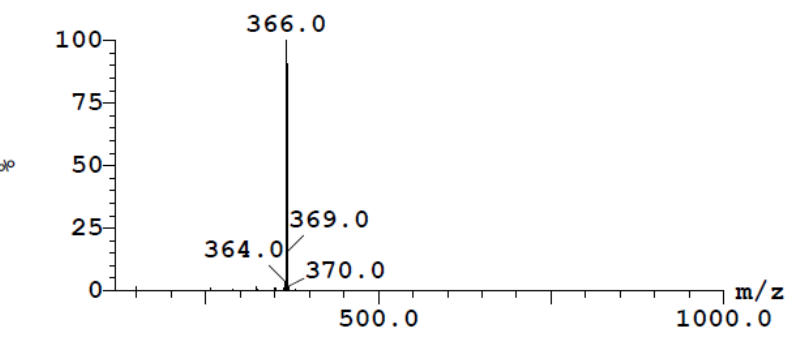




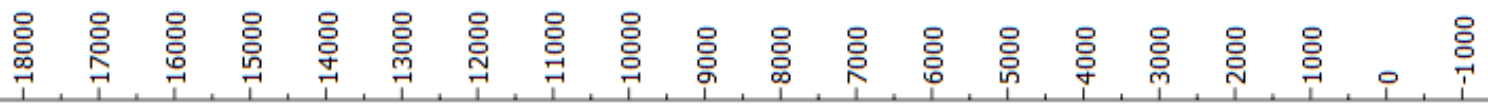

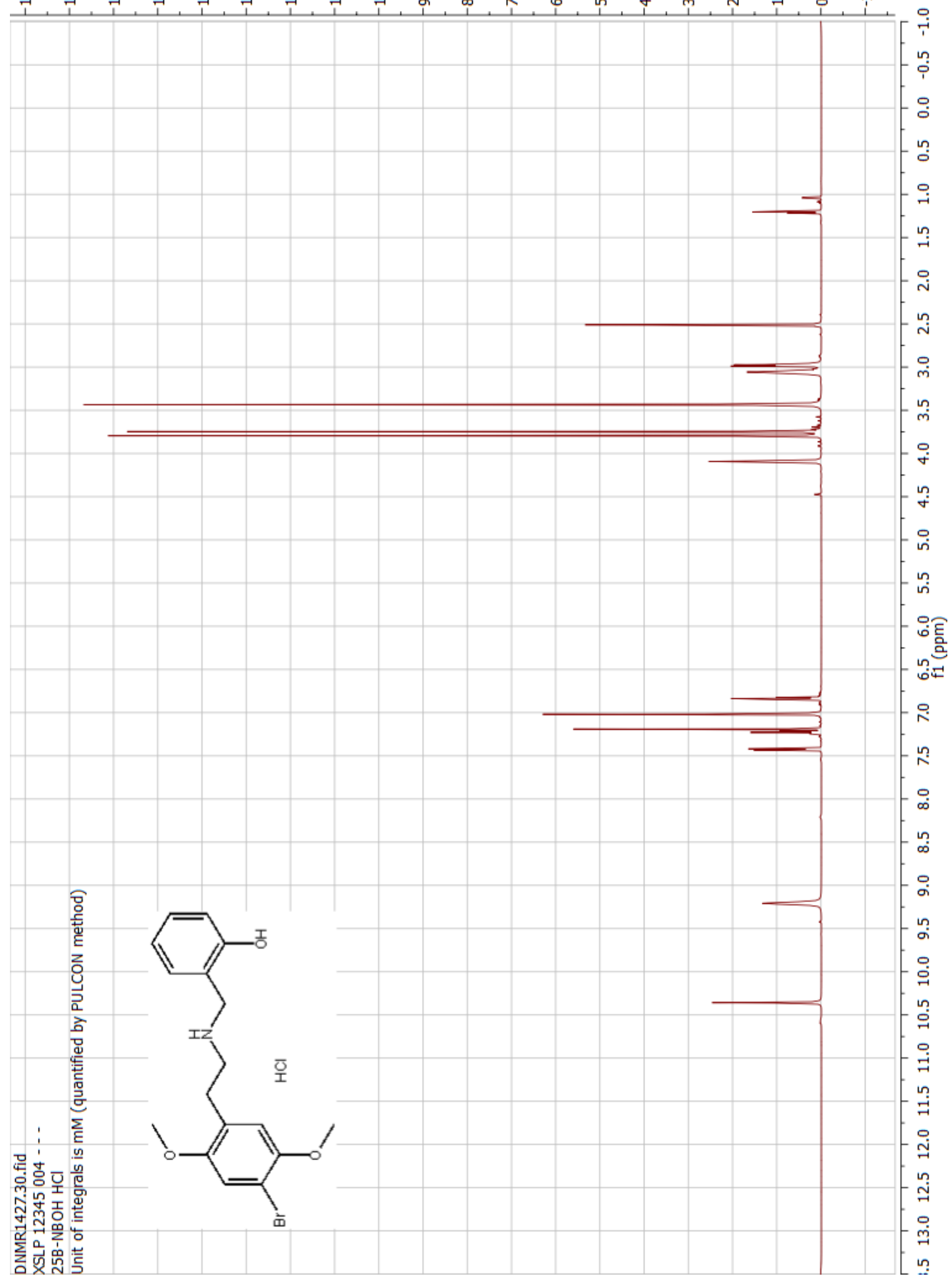




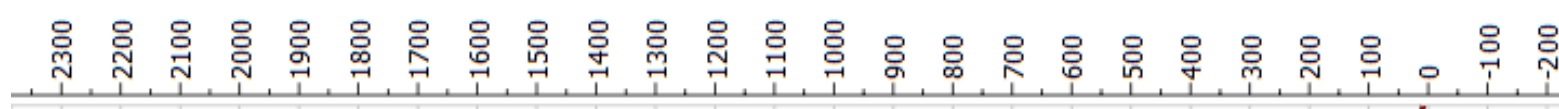

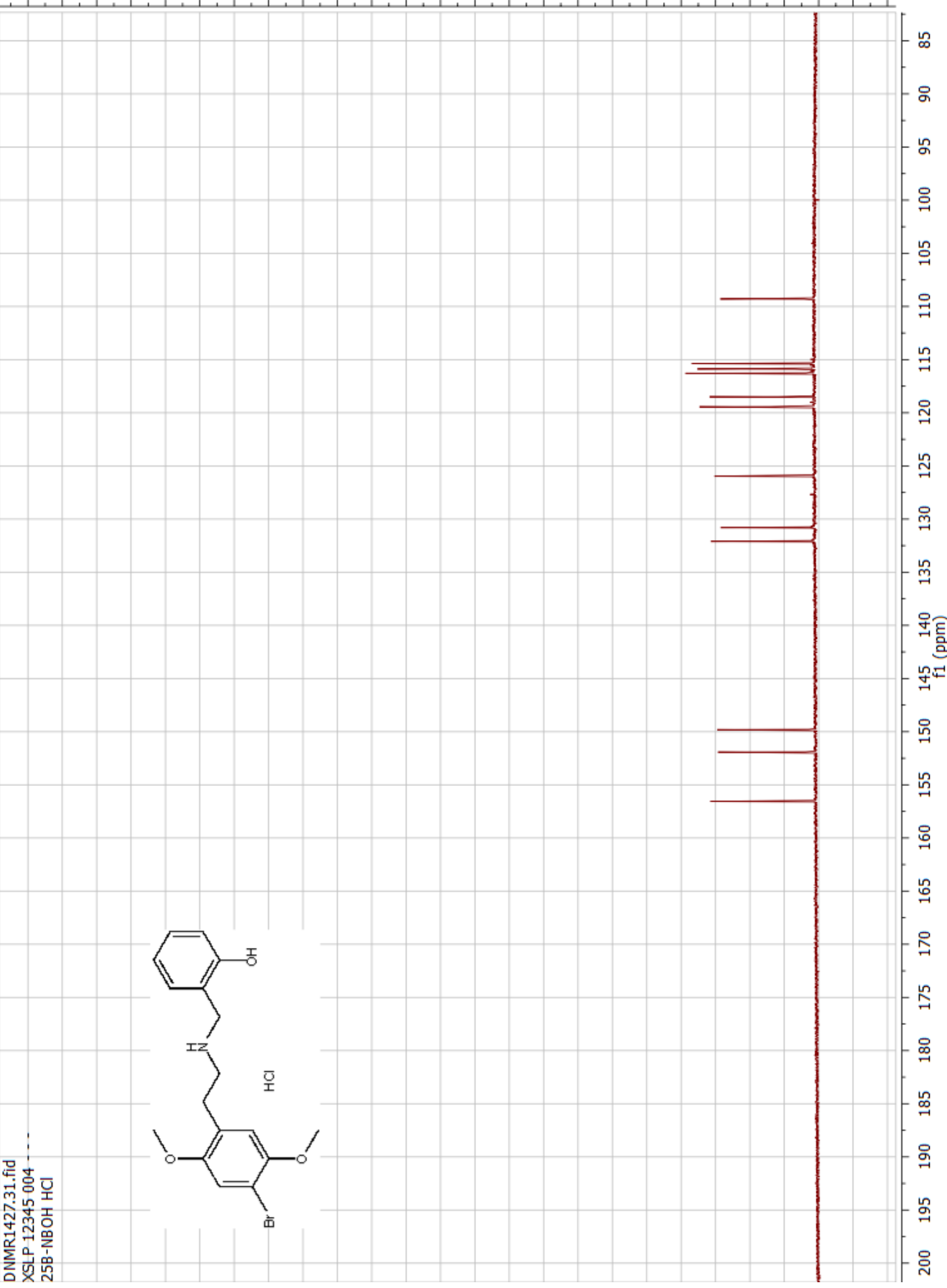


File:25B-NBOH

Printed: Mon Jul 21 13:40:16 2014

Sample Report:

Sample 1 Vial 1:4 ID 25B-NBOH File 25B-NBOH Date 21-Jul-2014 Time 13:34:13 Description

1: MS APPI+: TIC Smooth (SG, $2 \times 2$

(4)

$6.8 e+008$
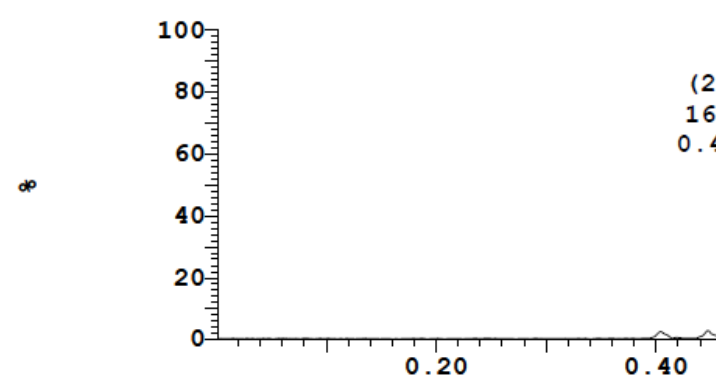

618

0.51

(2)

168

0.49

(2) PDA Ch1 254nme1.2nm

(4)
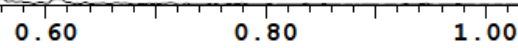

1.20

1. $228 e-1$

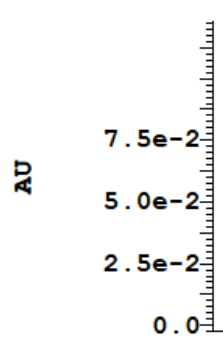

0.51

Range: $1.253 e-1$

(3) ELSD Signal

0.40

0.60

0.80

1.00

1.20

(2)

$(4)$

1131.472

750.000青

42 .

Range: 1131.743

: 52 
File:25B-NBOH

Date:21-Jul-2014

ID.25B-NBOH

Time:13:34:13

Description:

Inlet Method:Method452

Printed: Mon Jul 21 13:40:16 2014

Sample Report (continued):

Peak ID Compound Time Mass Found 0.49

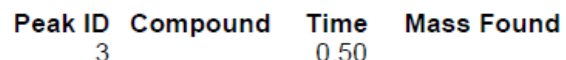

$1:$ MS APPI+

7. $5 e+006$

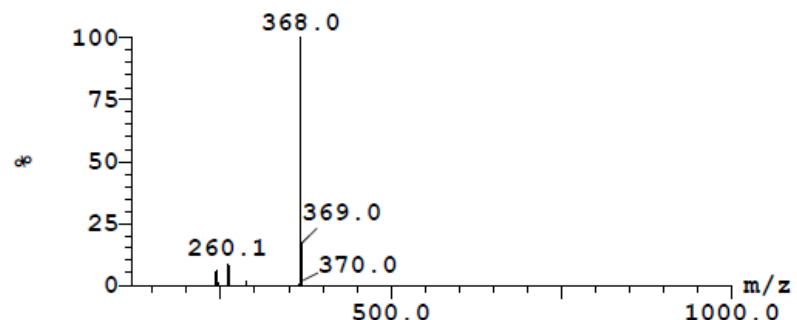

Peak ID Compound Time Mass Found

$4 \quad 0.51$

1000.0

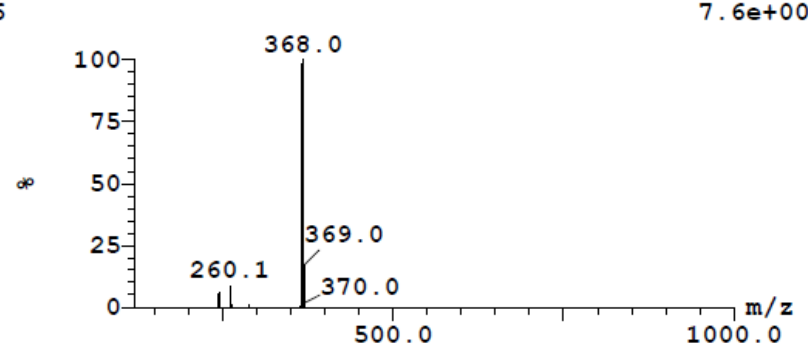

1 :MS APPI+

. $6 e+006$

1 :MS APPI+

7. $6 \mathrm{e}+006$

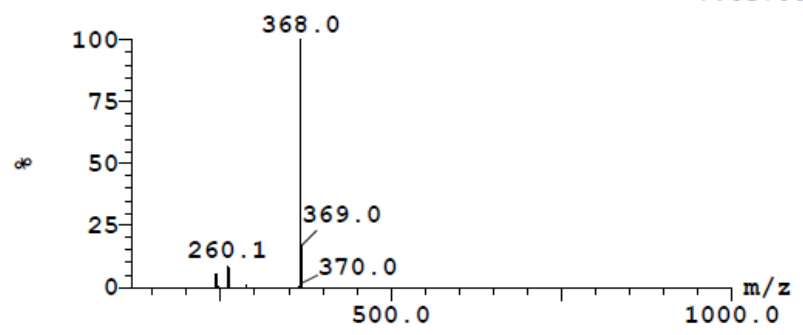


Printed: Mon Jul 21 12:30:06 2014

Sample Report:

Sample 1 Vial 1:39 ID 2-OGlu-F23 File 2-OGlu-F23 Date 21-Jul-2014 Time 12:00:01 Description

1: MS APPI+ :TIC Smooth (SG, $2 \times 2)$

(4)

088

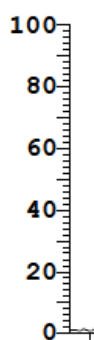

158
0.63
)

$2.1 e+008$

(2) PDA Ch1 254nme1.2nm

0.20

0.40

$(4)$

0.80

1.00

1.20

888

4. $918 e-2$

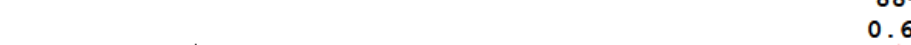

\section{.64}

Range: $5.143 e-2$

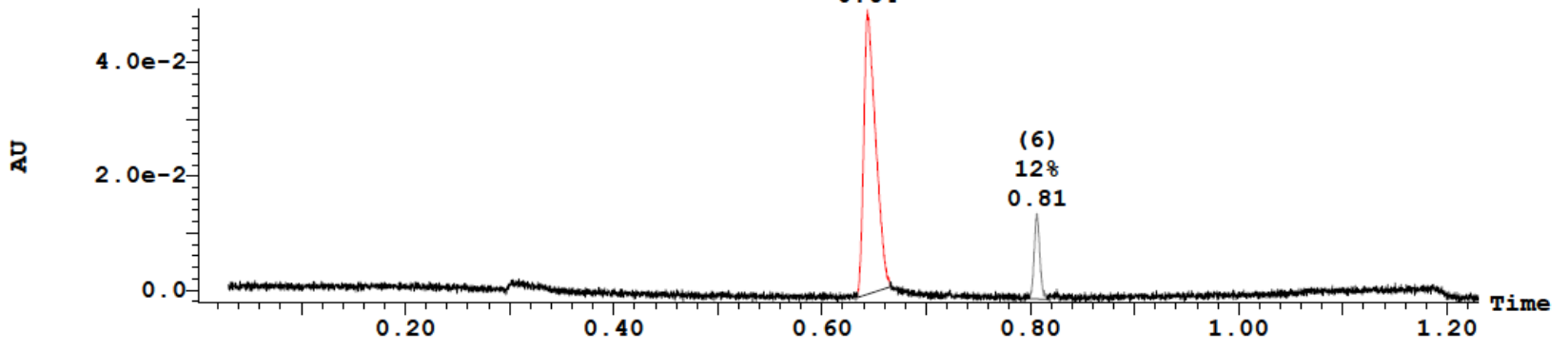

(3) ELSD Signal

(4)

1102.000

96
0.65

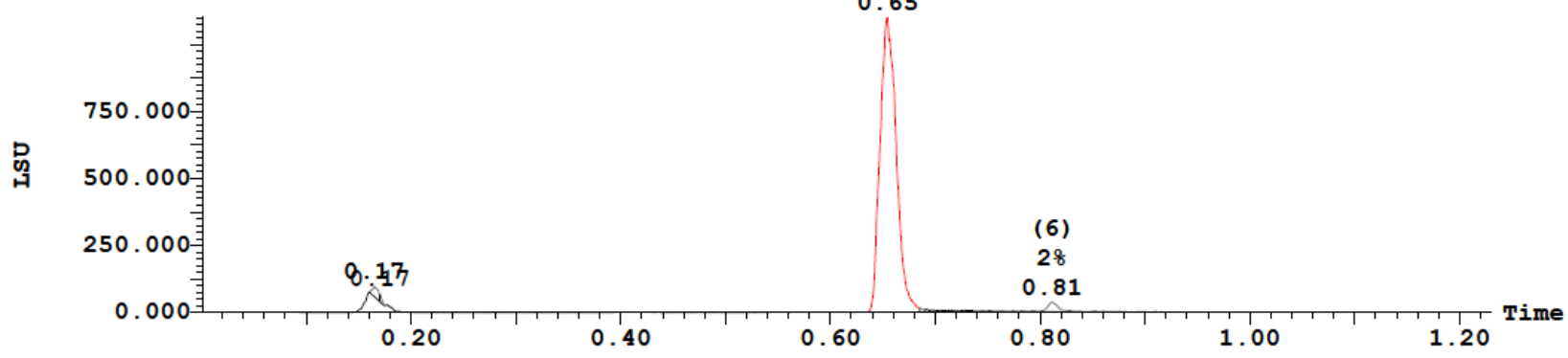


Printed: Mon Jul 21 12:30:06 2014

Sample Report (continued):

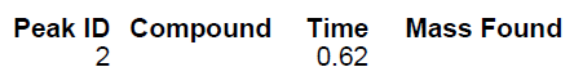

Peak ID Compound Time
3

$1:$ MS APPI+

9. $7 e+005$
Mass Found

$1:$ MS APPI+

1. $1 e+006$
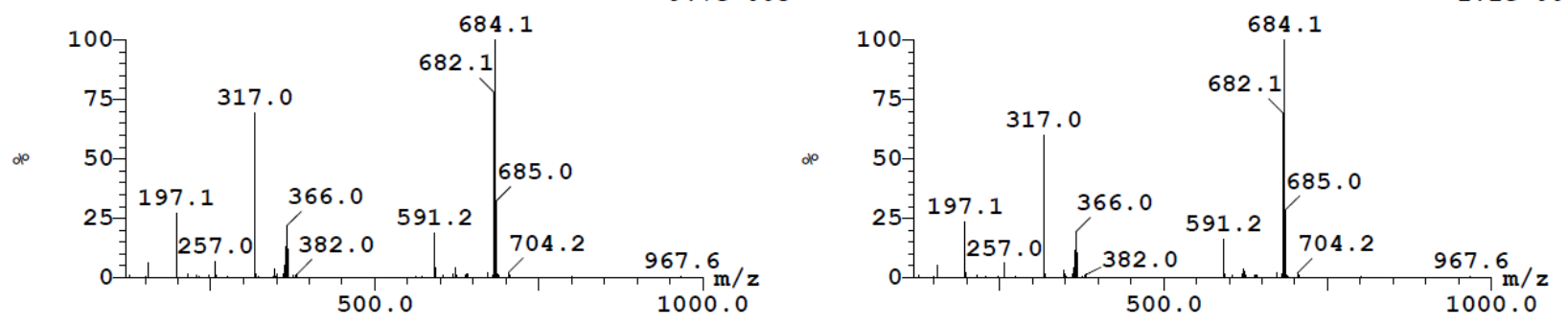

Peak ID Compound Time Mass Found

$1:$ MS APPI+

1. $2 e+006$

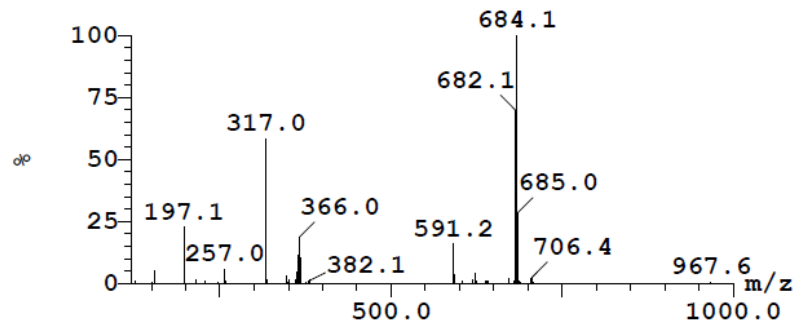


Sample Report:

Sample 1 Vial 1:8 ID NBOGluOAcF32 File NBOGluOAcF32 Date 24-Jul-2014 Time 13:53:31 Description

1: MS APPI+ :TIC Smooth (SG, 2x2)

(3)

$1.6 e+008$
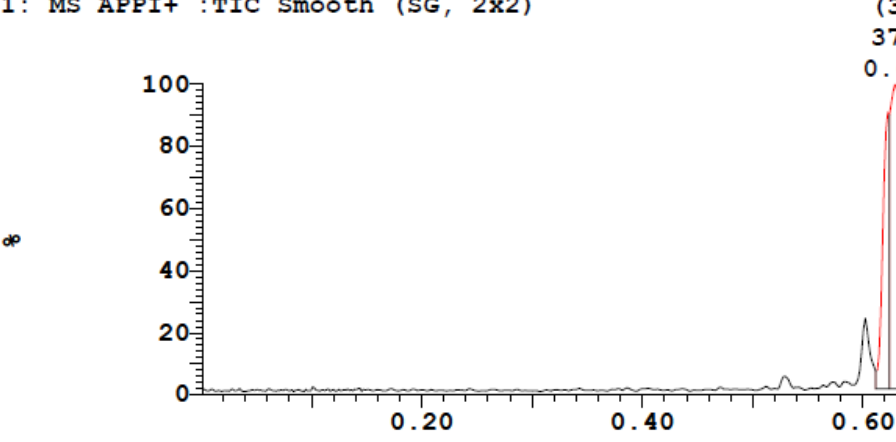

378

(2) PDA Ch1 254nme1.2nm

0.60

0.80

1.00

1.20

(4)

7. $713 e-2$

988
.64

Range: $7.953 e-2$

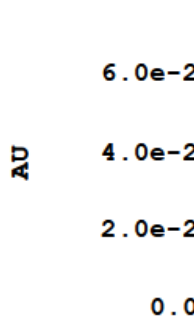

(3) ELSD Signal

0.20

(2) $(2)$

$0.62^{28}$

.00

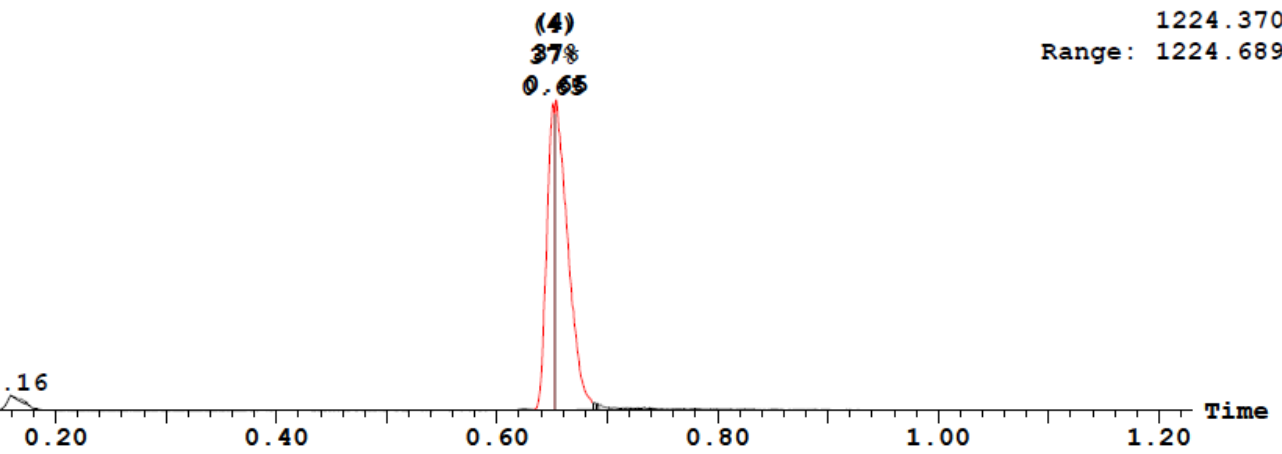


Printed: Thu Jul 24 13:59:38 2014

Sample Report (continued):

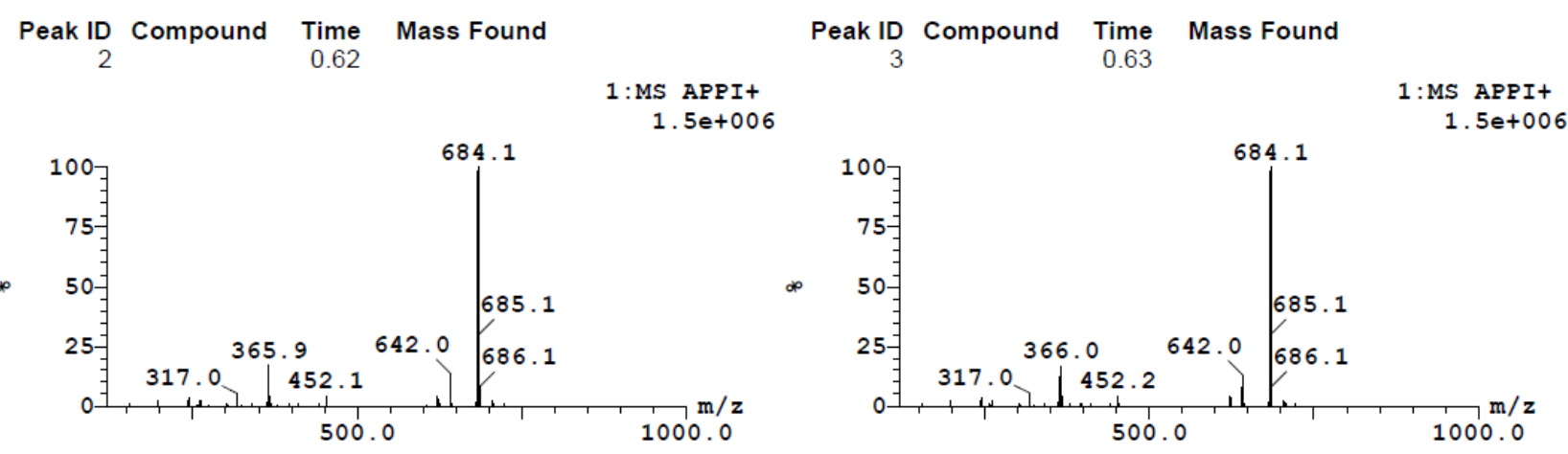

Peak ID Compound Time Mass Found

4 Compound

$1:$ MS APPI+

1. $6 e+006$

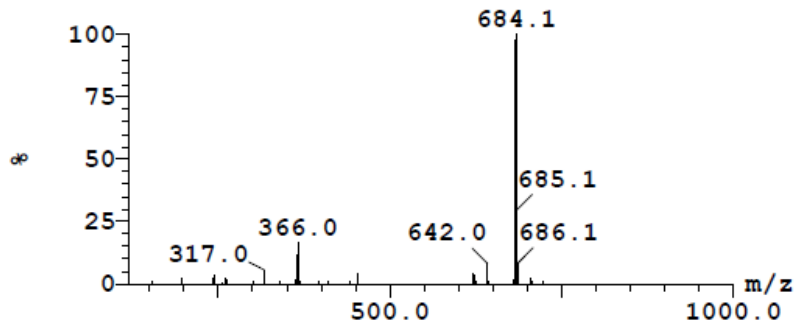




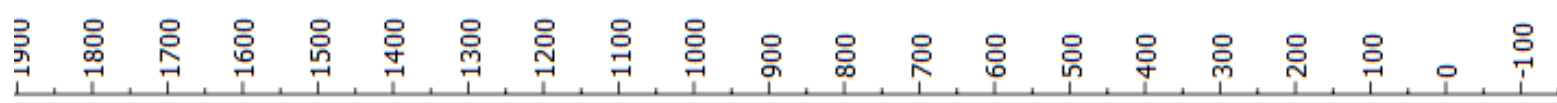

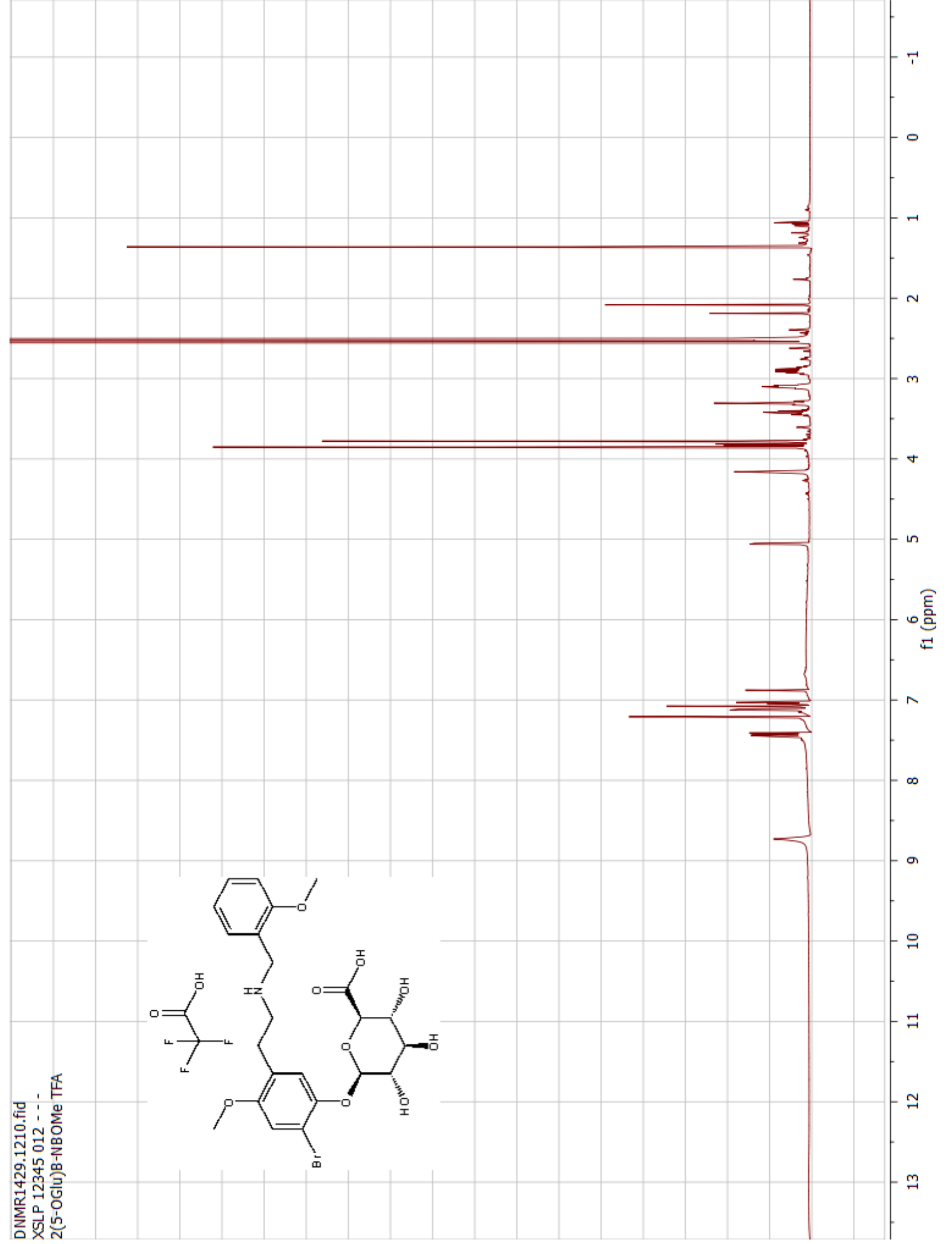




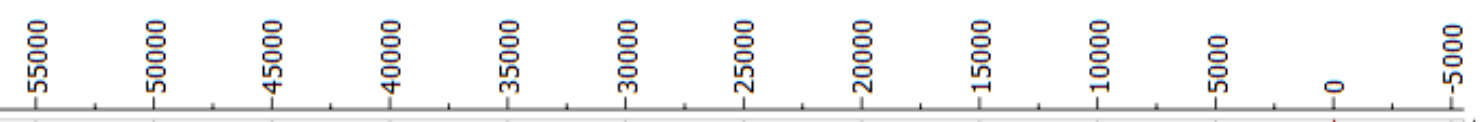

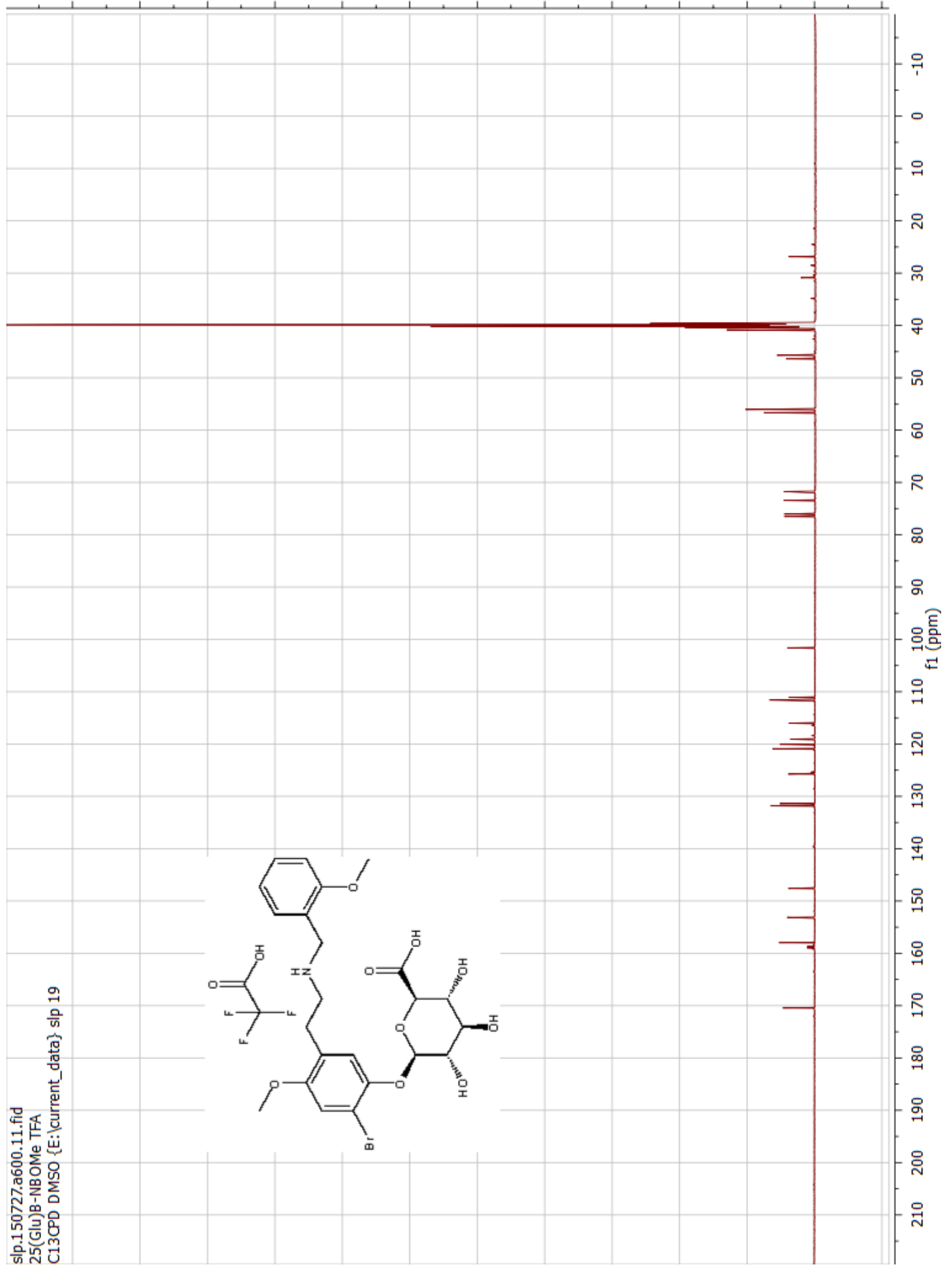


Printed: Fri Jul 18 12:59:46 2014

Sample Report:

Sample 1 Vial 1:40 ID 2_5-OGlu_B-NBOMe File 2_5-OGlu_B-NBOMe Date 18-Jul-2014 Time 12:51:39 Description

1: MS APPI+ :TIC Smooth (SG, 2x2) (1)
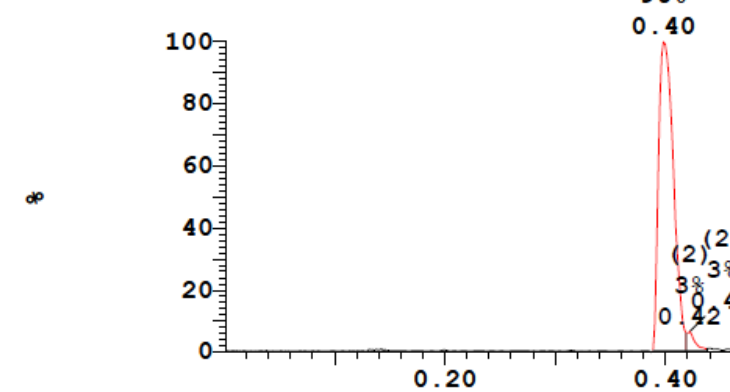

$$
)_{38}^{(2)}
$$

$\mathbb{1}_{2} 42$

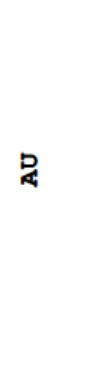

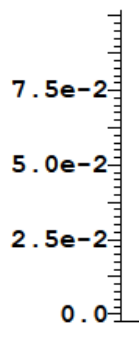

(3) ELSD Signal 
Description

Printed: Fri Jul 18 12:59:46 2014

Sample Report (continued):

\section{Peak ID Compound Time Mass Found 0.40}

Peak ID Compound $\begin{array}{r}\text { Time } \\ 2\end{array} \quad$ Mass Found
0.42

1:MS APPI+

2. $3 e+006$
1:MS APPI+

2. $3 e+006$

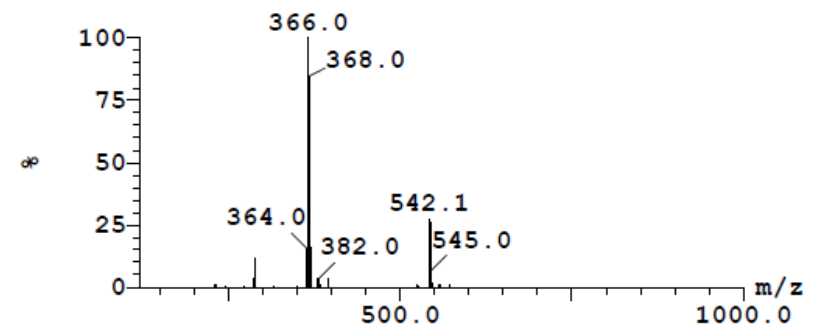

$\begin{array}{rrr}\text { Peak ID Compound } \begin{array}{r}\text { Time } \\ 6\end{array} & \text { Mass Found } \\ 0.48 & \end{array}$ 1000.0

1 :MS APPI+

2. $2 e+004$

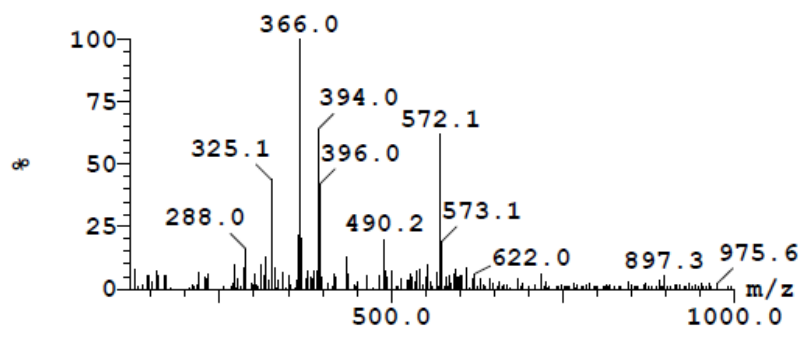




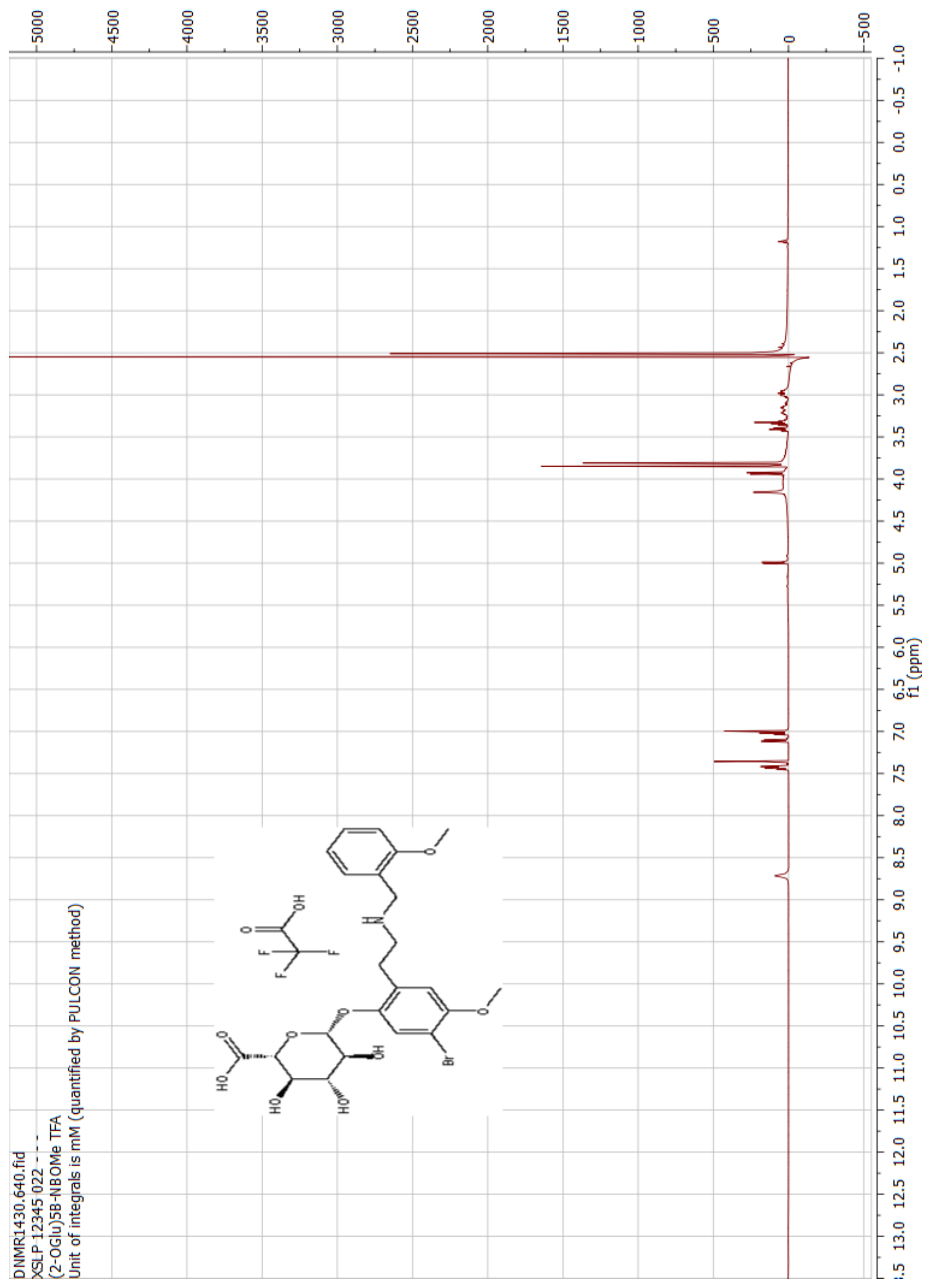




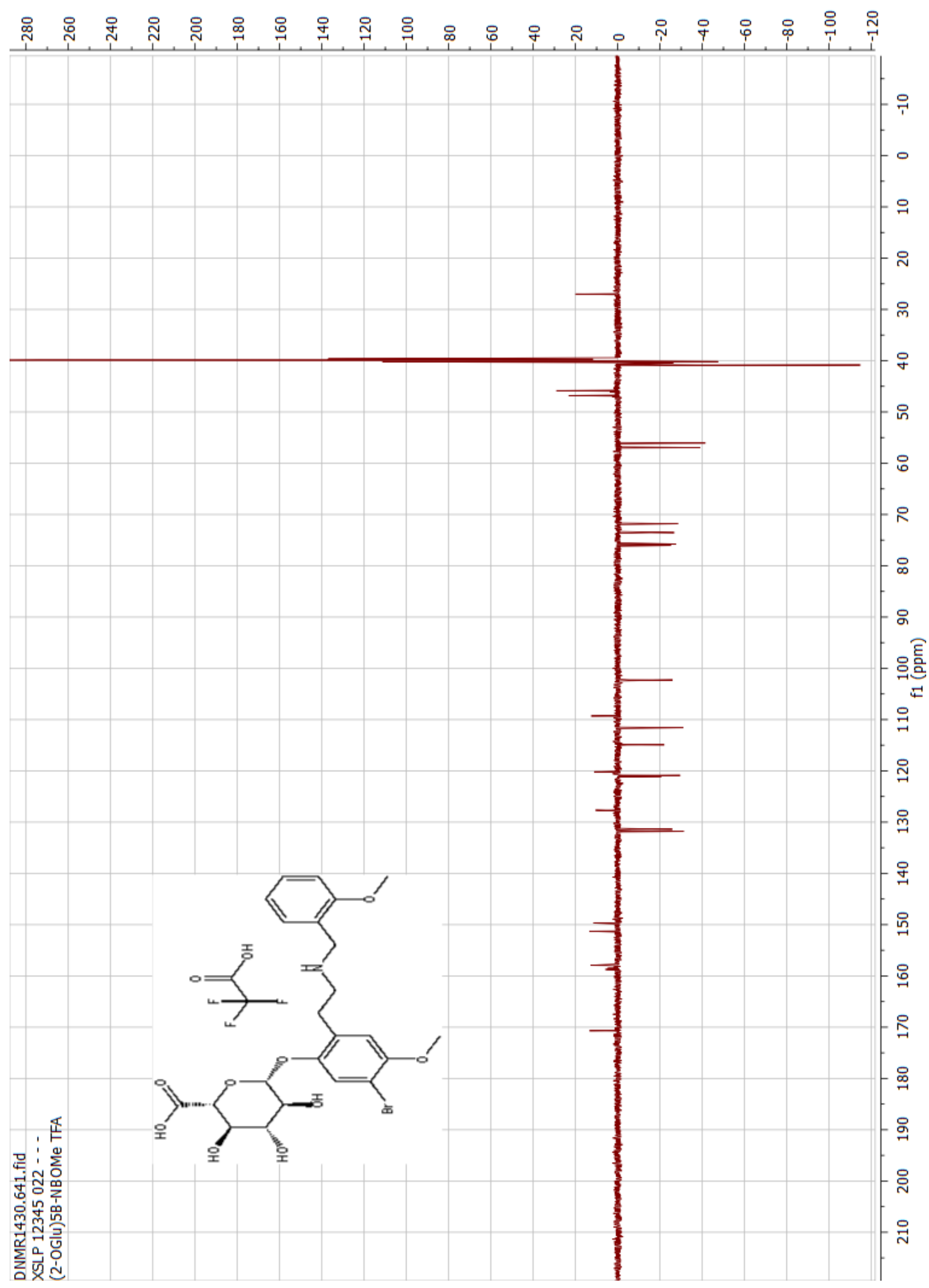


Printed: Tue Jul 22 12:18:50 2014

\section{Sample Report:}

\section{Sample 1 Vial 1:13 ID 2-OGlu-5B-NBOMePrepF1 File 2-OGlu-5B-NBOMePrepF1 Date 22-Jul-2014 Time 12:00:20 Description}

1: MS APPI+ :TIC Smooth (SG, 2x2) (1)

1. $4 e+007$

778
0.41

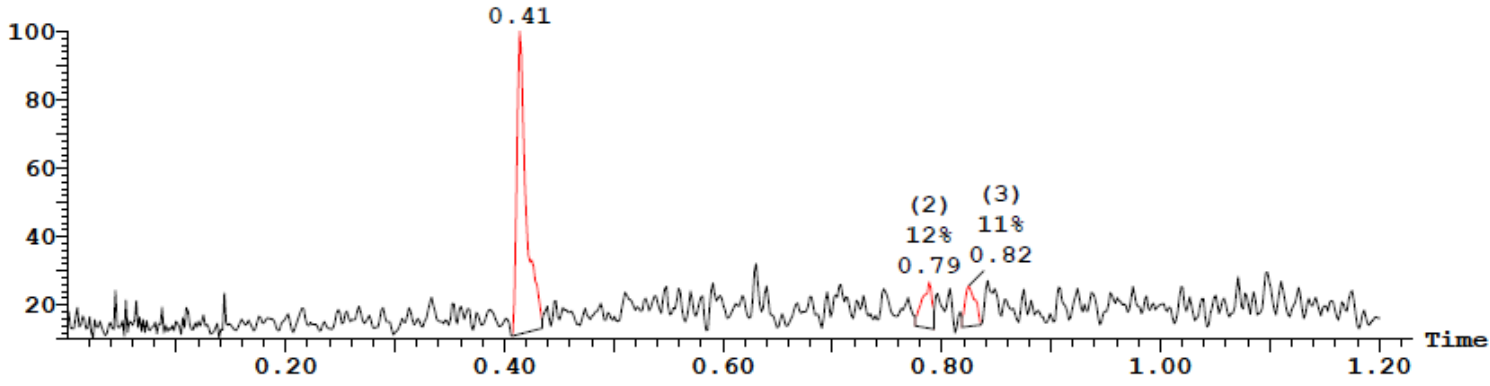

(2) PDA Ch1 254nme1.2nm
$100 \%$

$\begin{array}{ll}\text { (2) PDA Ch1 } 254 \mathrm{~nm} 1.2 \mathrm{~nm} & \text { (1) } \\ & 1008\end{array}$

1. $074 \mathrm{e}-2$

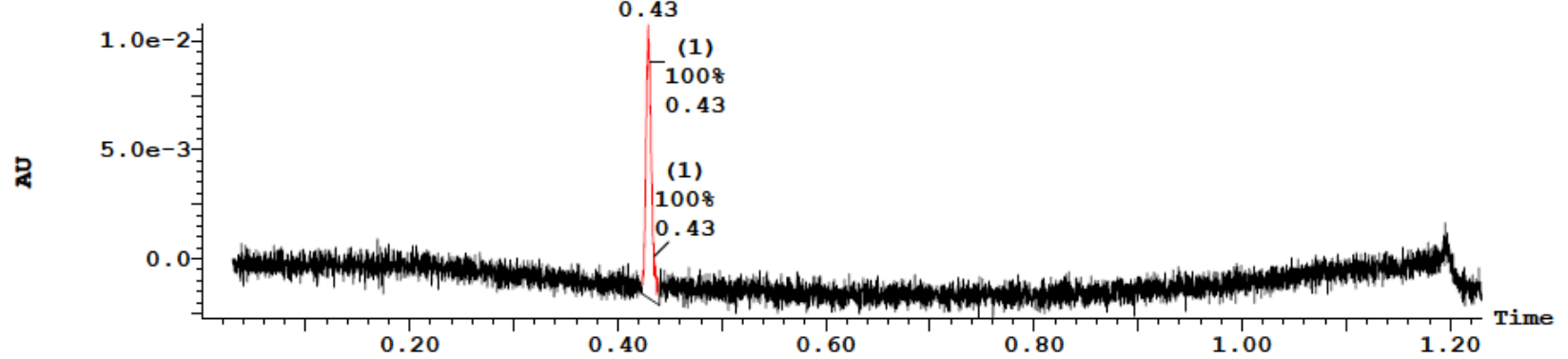

(3) ELSD Signal

(1)

16.925

1008
0.44

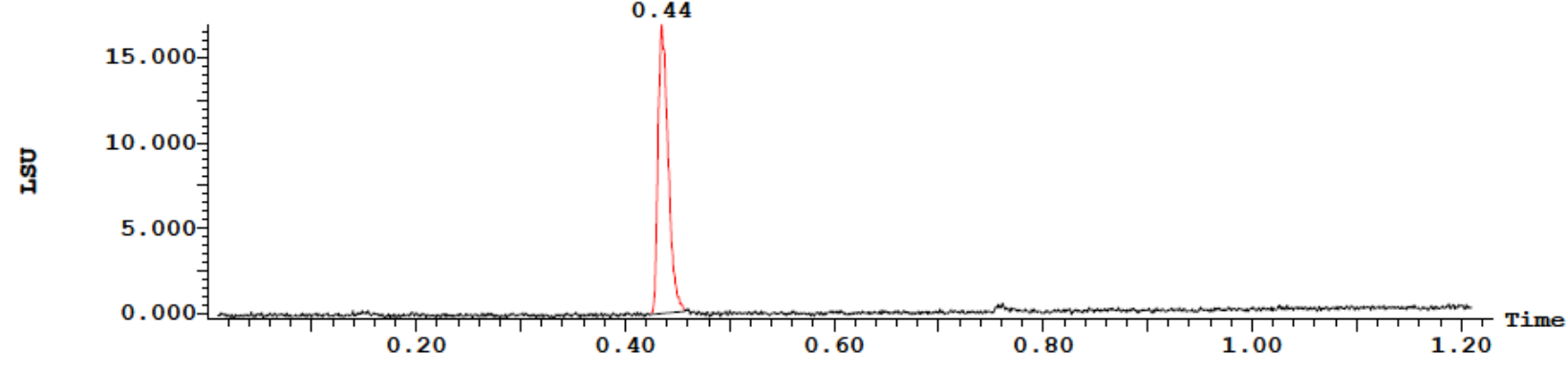


Openlynx Report - XSLP

Sample: 1

File:2-OGlu-5B-NBOMePrepF1

Description:

Printed: Tue Jul 22 12:18:50 2014
Vial:1:13

Date:22-Jul-2014

Inlet Method:Method452
ID:2-OGlu-5B-NBOMePrepF1

Time:12:00:20

Sample Report (continued):

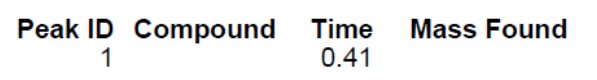

1:MS APPI+

3. $0 e+004$

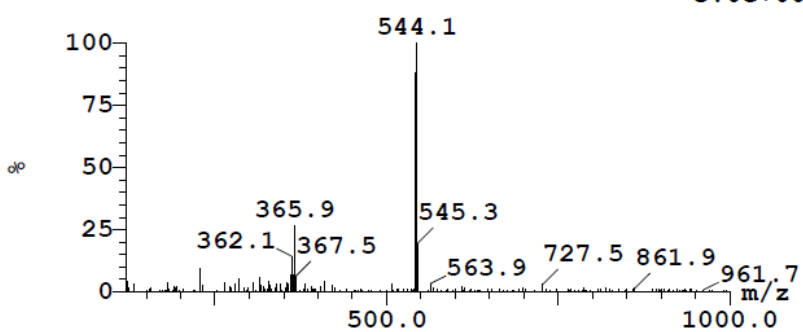

Peak ID Compound Time Mass Found

3 0.82
Peak ID Compound Time

0.79

Mass Found

1 :MS APPI+

3. $2 e+003$

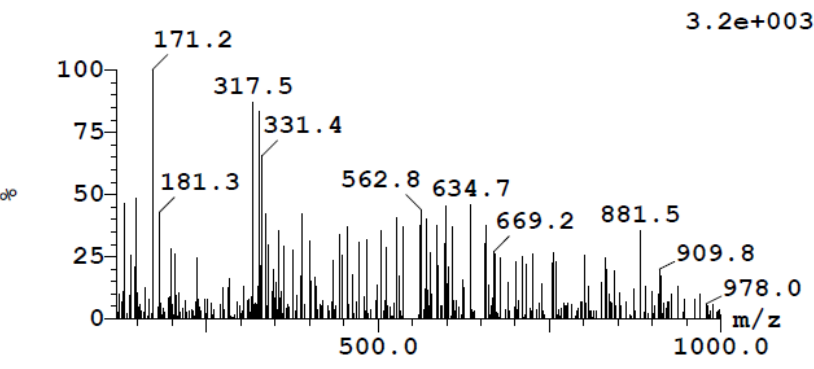

1:MS APPI+

2. $4 e+003$

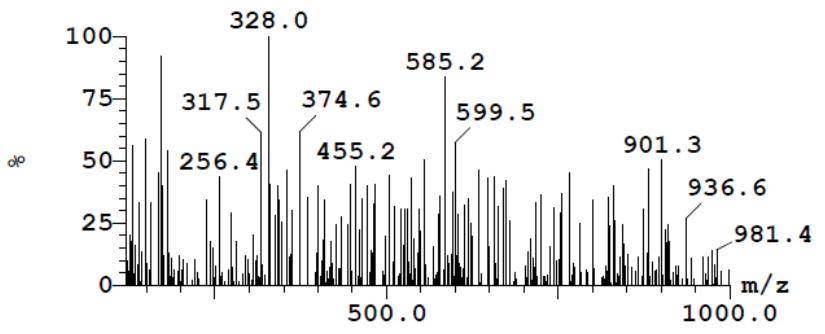




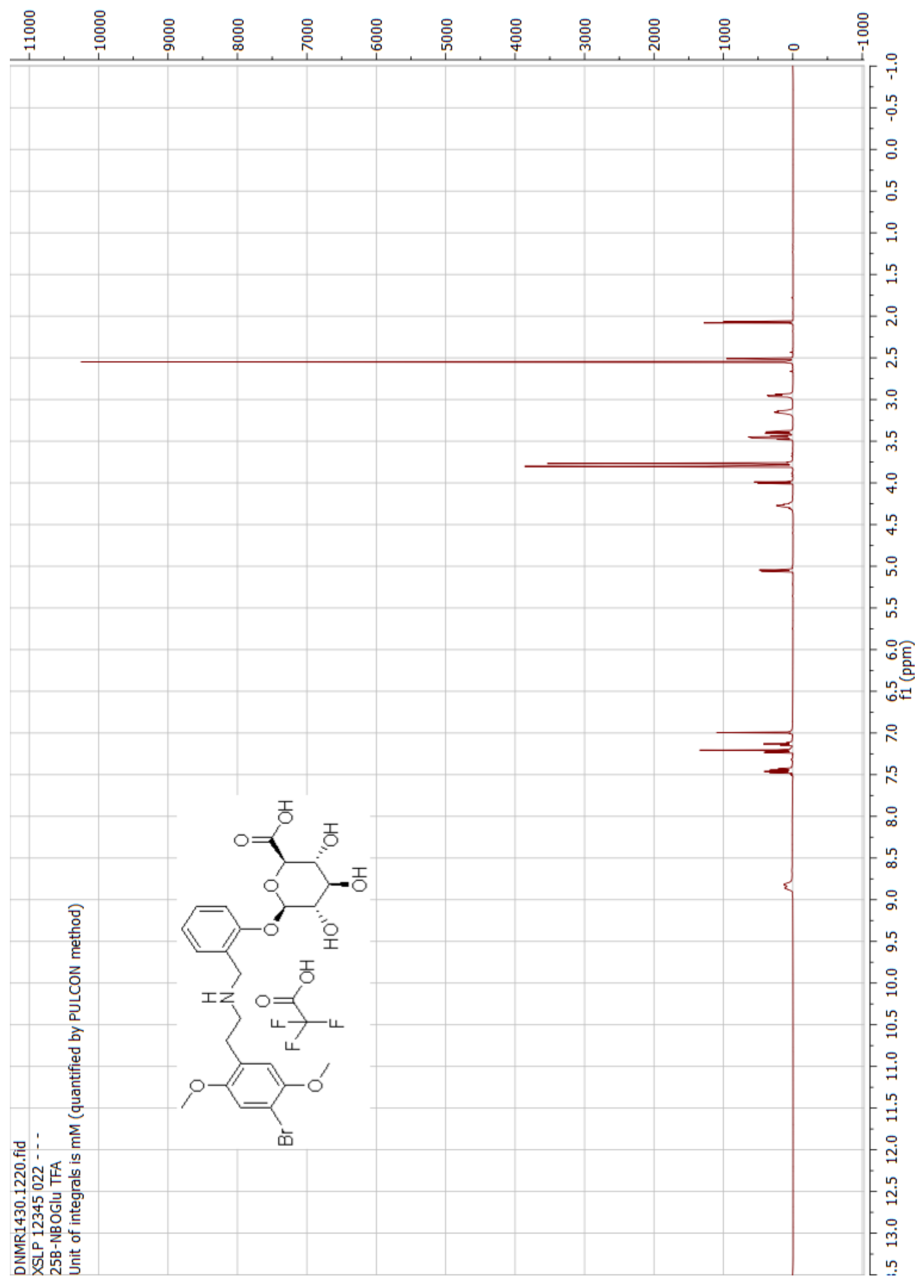




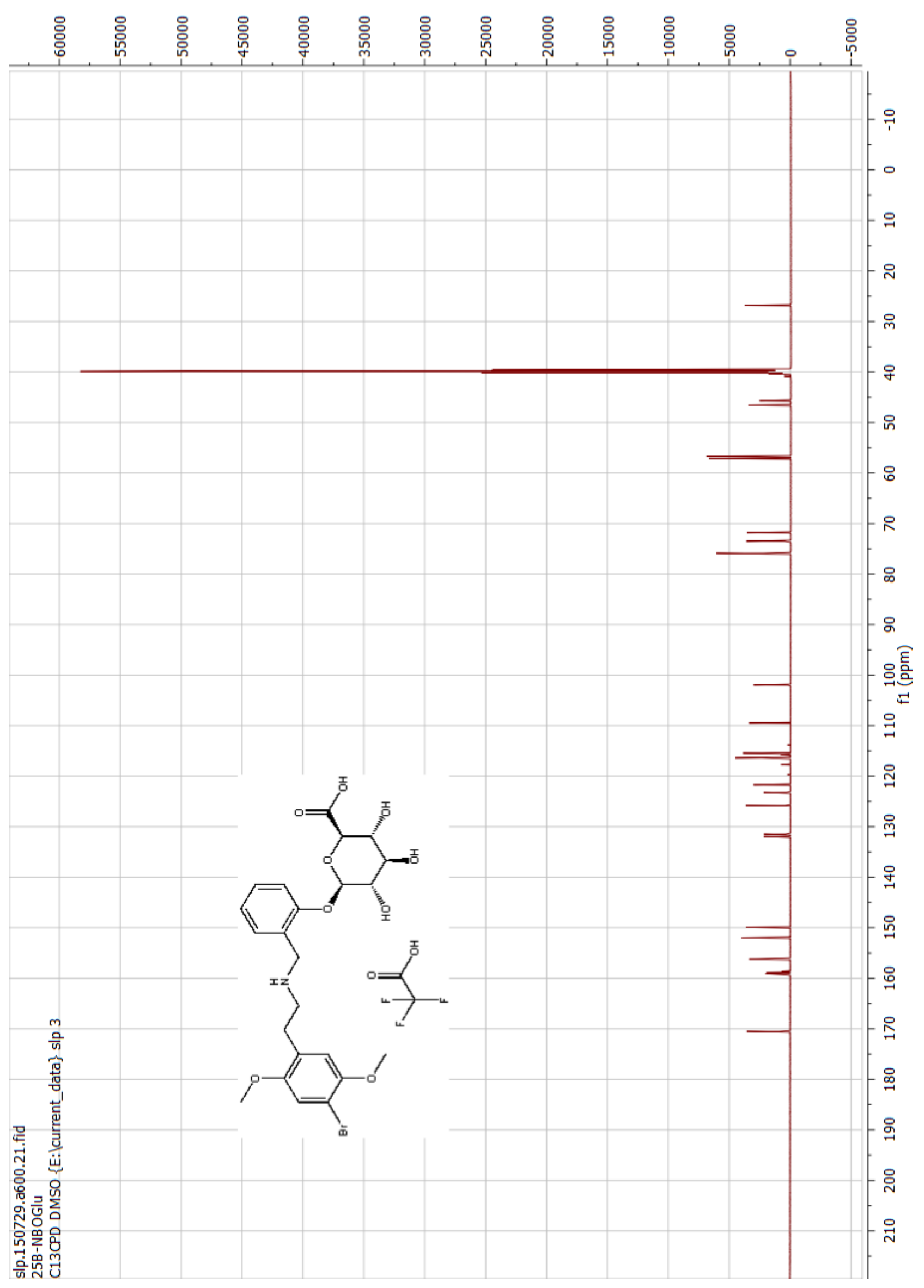


Openlynx Report - XSLP

Sample: 1

Vial:1:40

Date:25-Jul-2014
Inlet Method:Method4

ID:25B-NBOGlu-final

File:25B-NBOGlu-final

Description:

Printed: Fri Jul 25 08:13:17 2014

Sample Report:

Sample 1 Vial 1:40 ID 25B-NBOGlu-final File 25B-NBOGlu-final Date 25-Jul-2014 Time 08:01:26 Description

1: MS APPI+ :TIC Smooth (SG, 2x2)

(1)

3. $4 e+007$
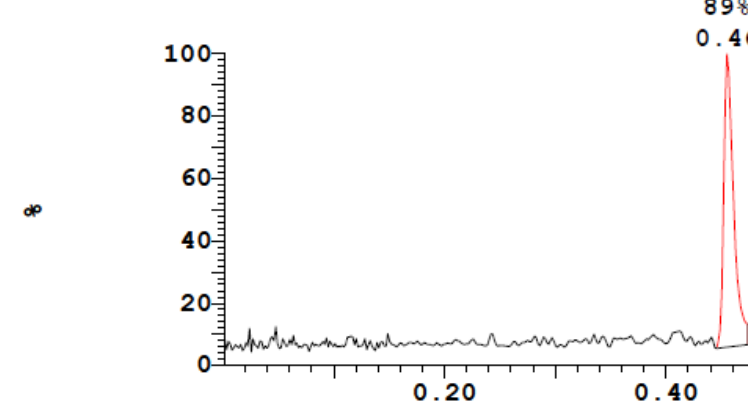

0.46

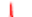

$(3)$

(3)

0.61

48

Time:08:01:26

1.03

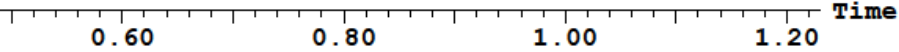

(2) PDA Ch1 254nme1.2nm

(1)

1. $735 e-2$

$100 \%$

Range: $1.984 \mathrm{e}-2$

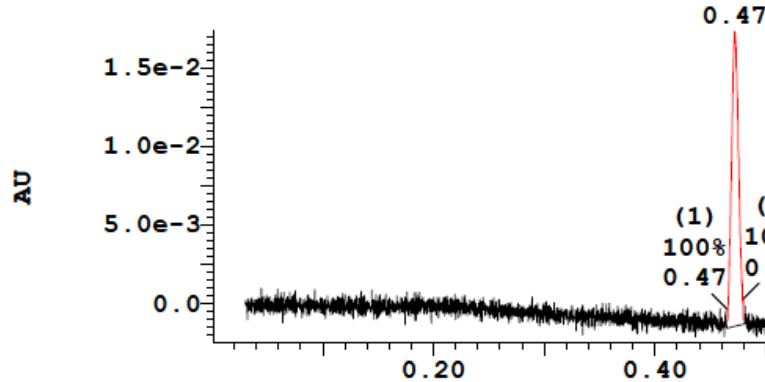

.47

(1) (1)

0.470 .48

(3) ELSD Signal

0.40

0.60

0.80

1.00

1. 20 Time

1008

30.000青

0.48

Range: 37.031

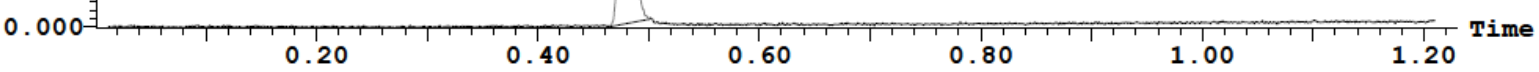


Printed: Fri Jul 25 08:13:17 2014

Sample Report (continued):

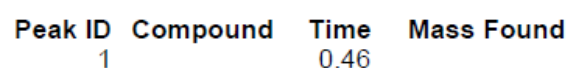

\section{$1:$ MS APPI+}

7. $4 \mathrm{e}+004$ $\begin{array}{rrr}\text { Peak ID Compound } & \text { Time } \\ 3 & 0.61 & \text { Mass Found }\end{array}$

$1:$ MS APPI+

5. $4 e+003$
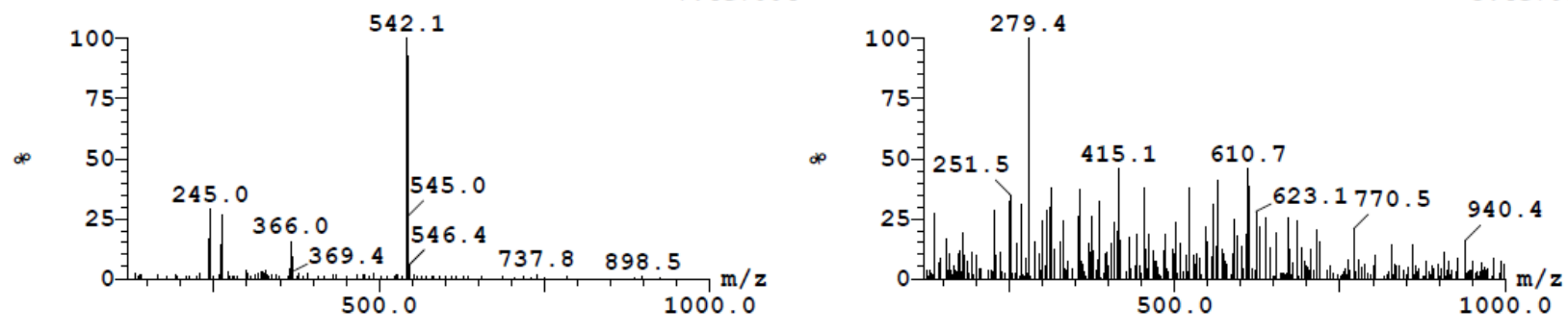

$$
\begin{array}{r}
\text { Peak ID Compound Time } \\
4
\end{array}
$$

\section{1:MS APPI+}

2. $7 e+003$

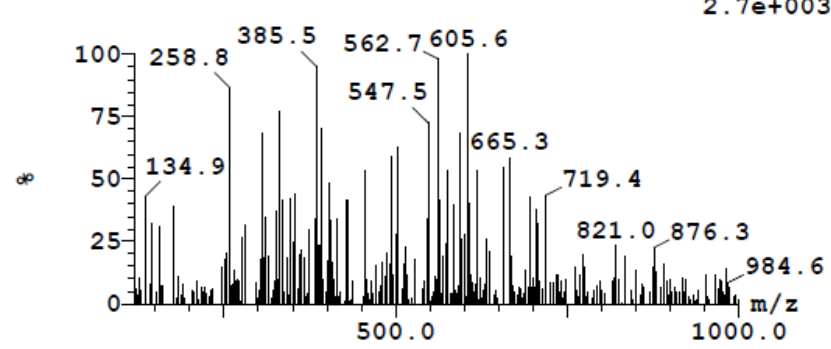




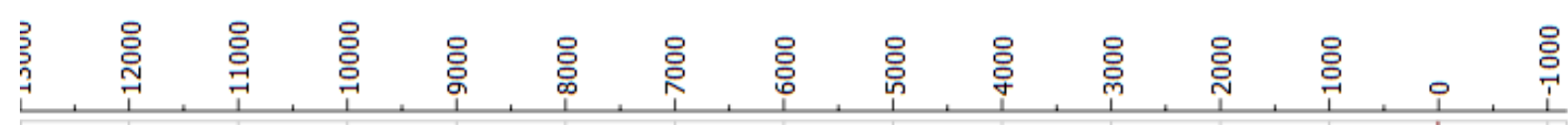

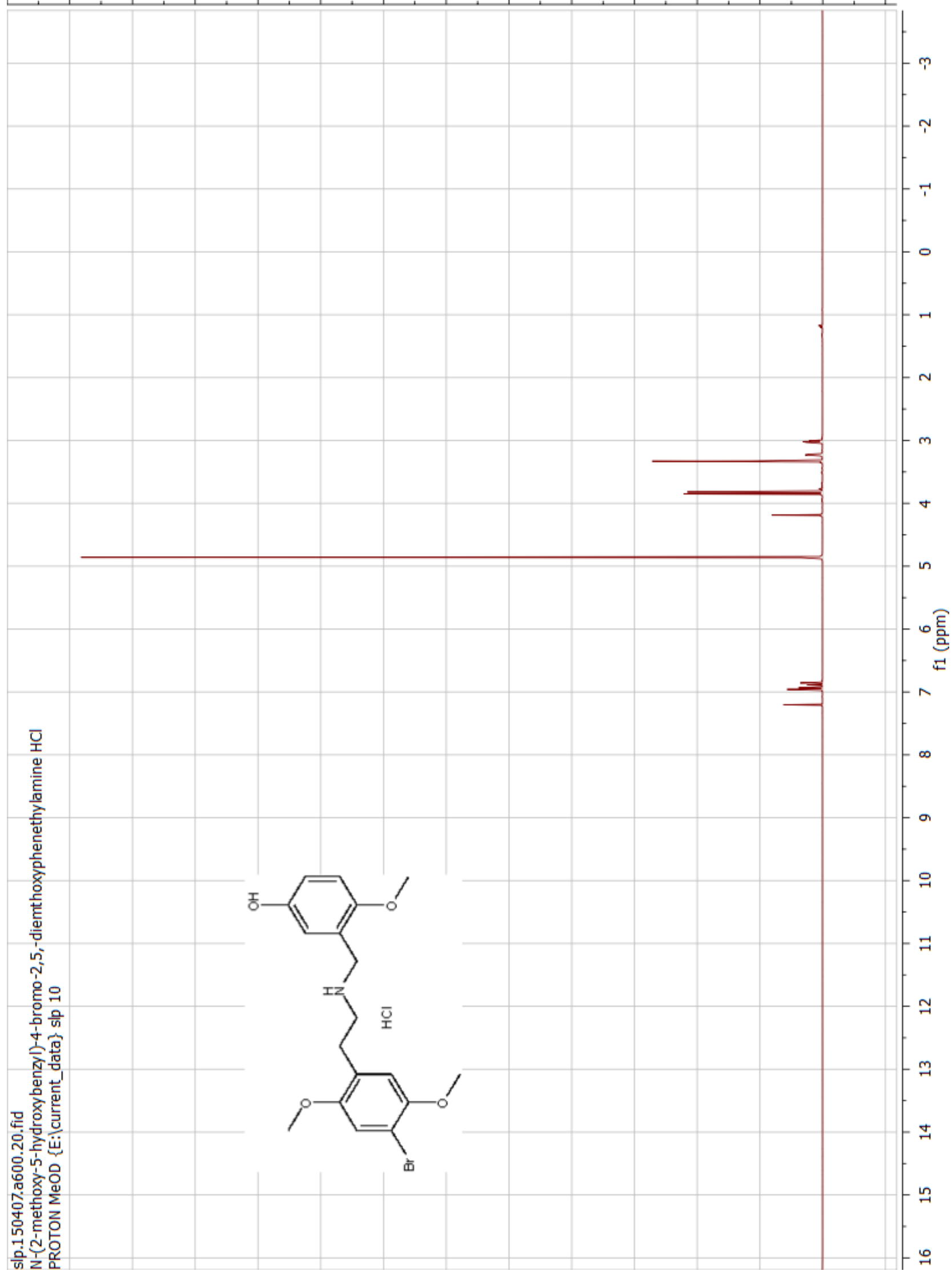




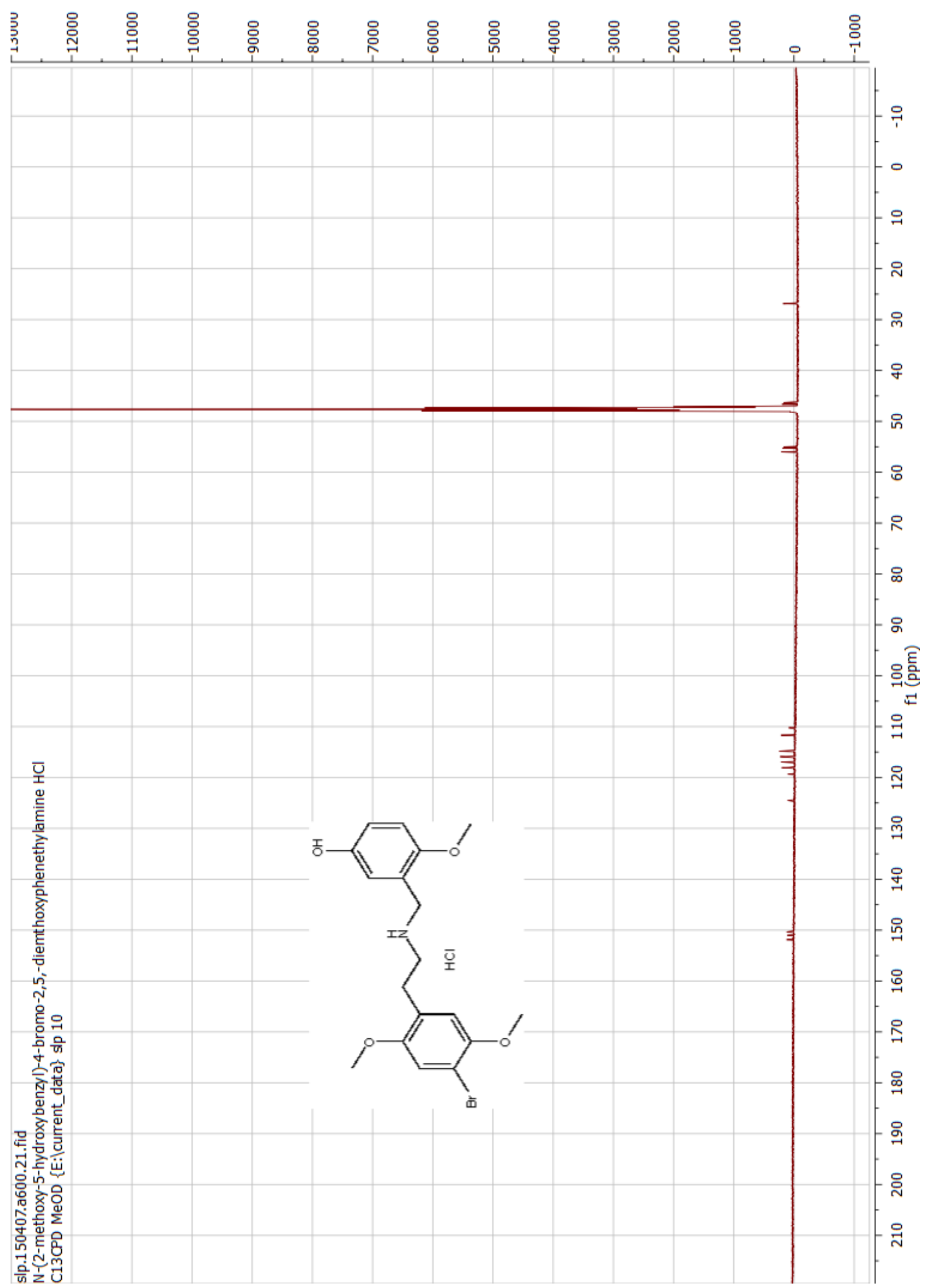




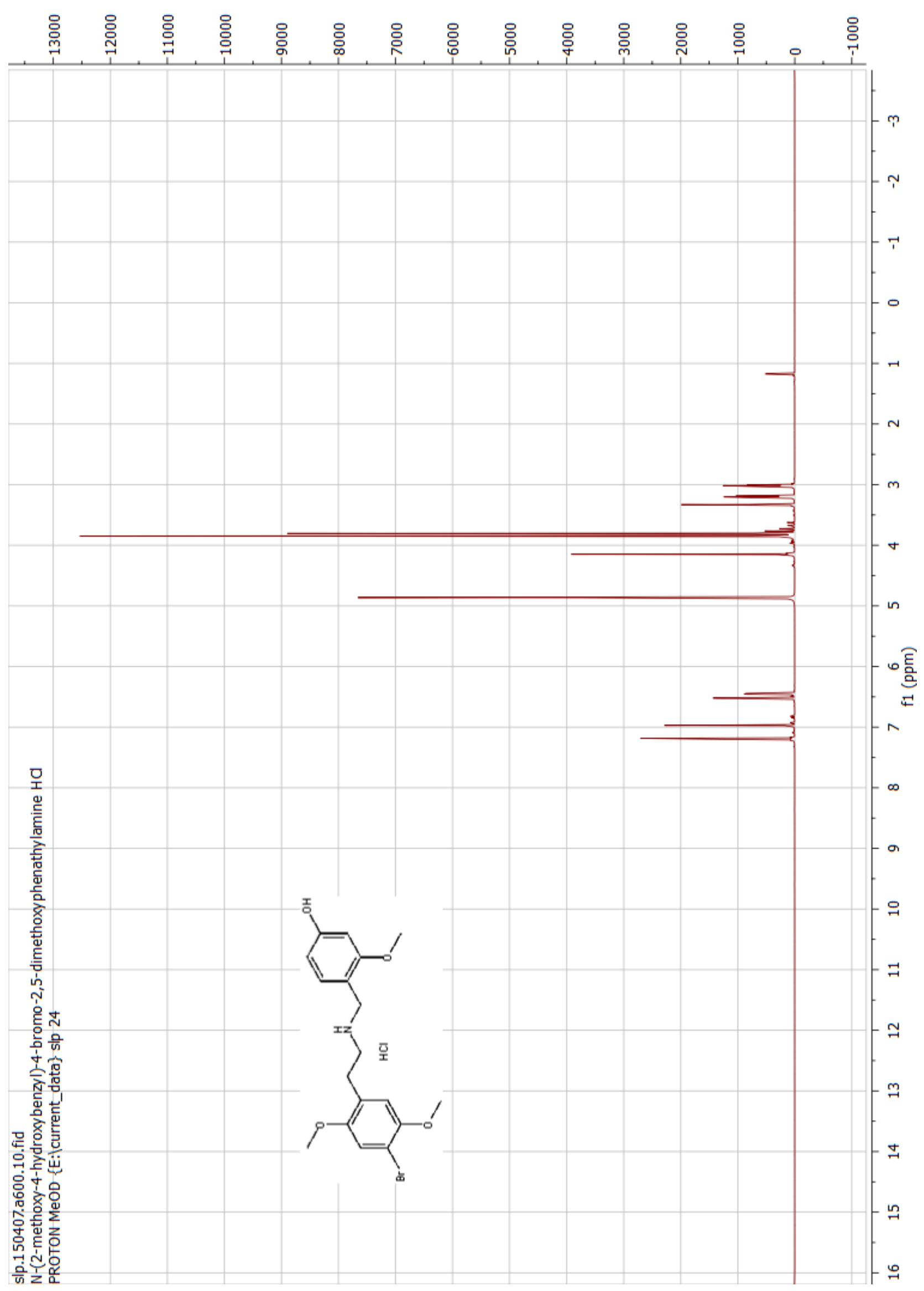




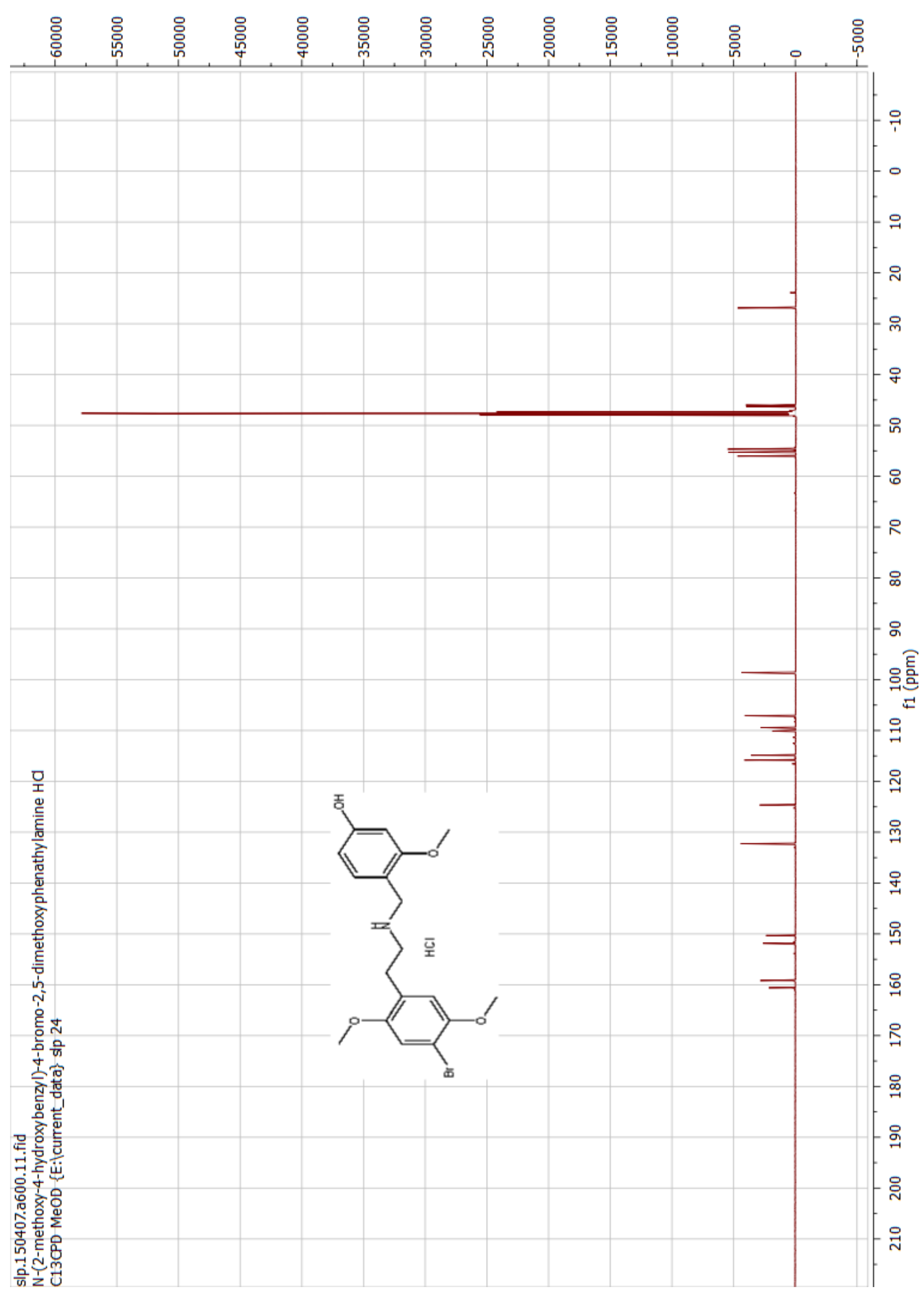




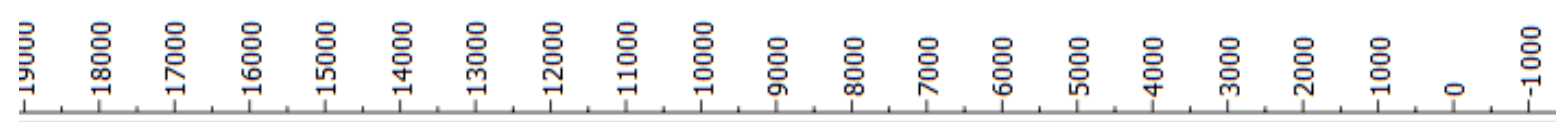

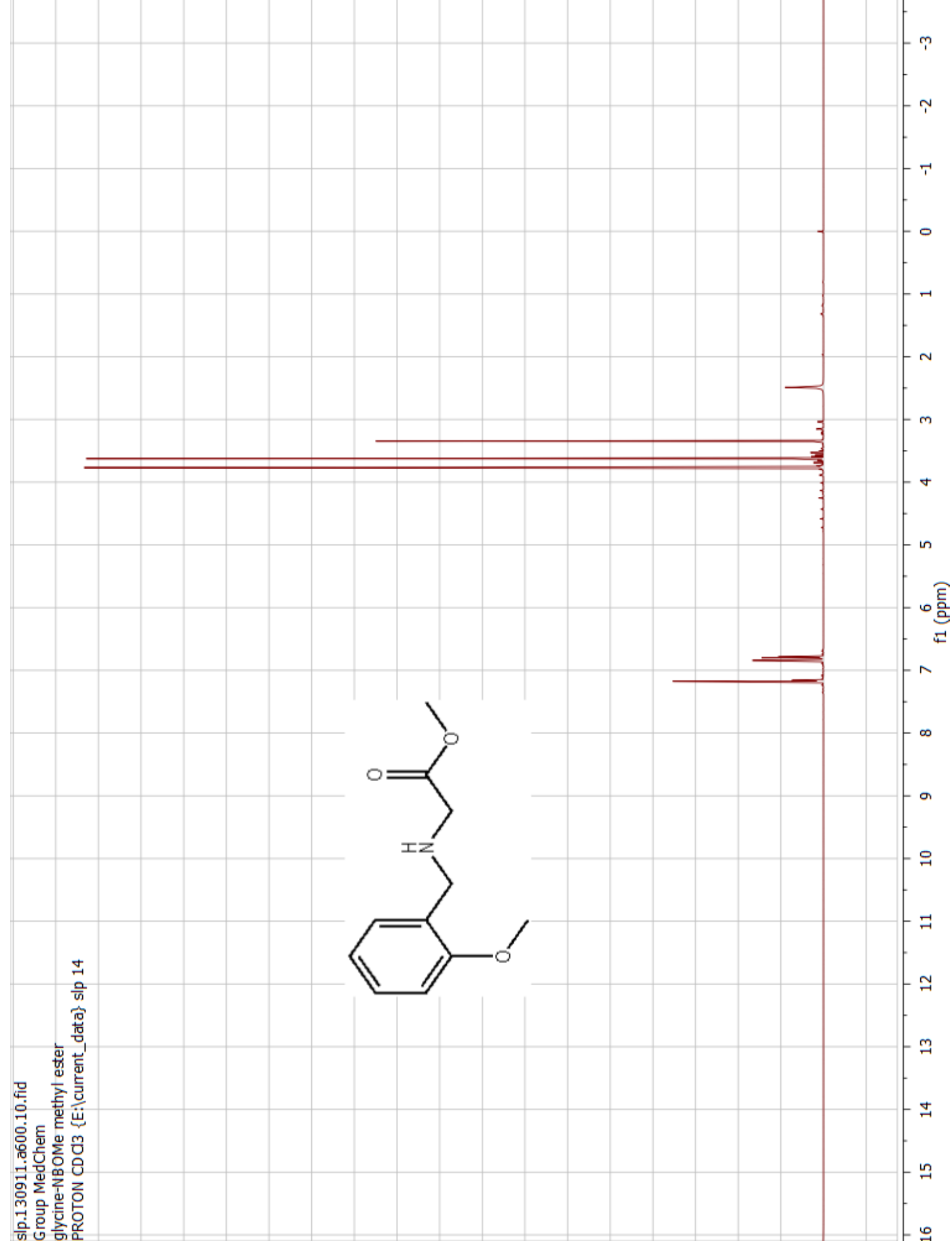




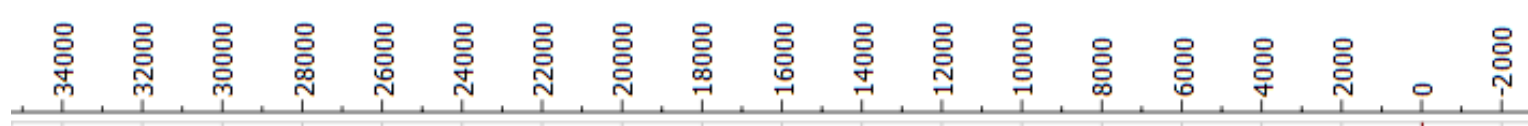

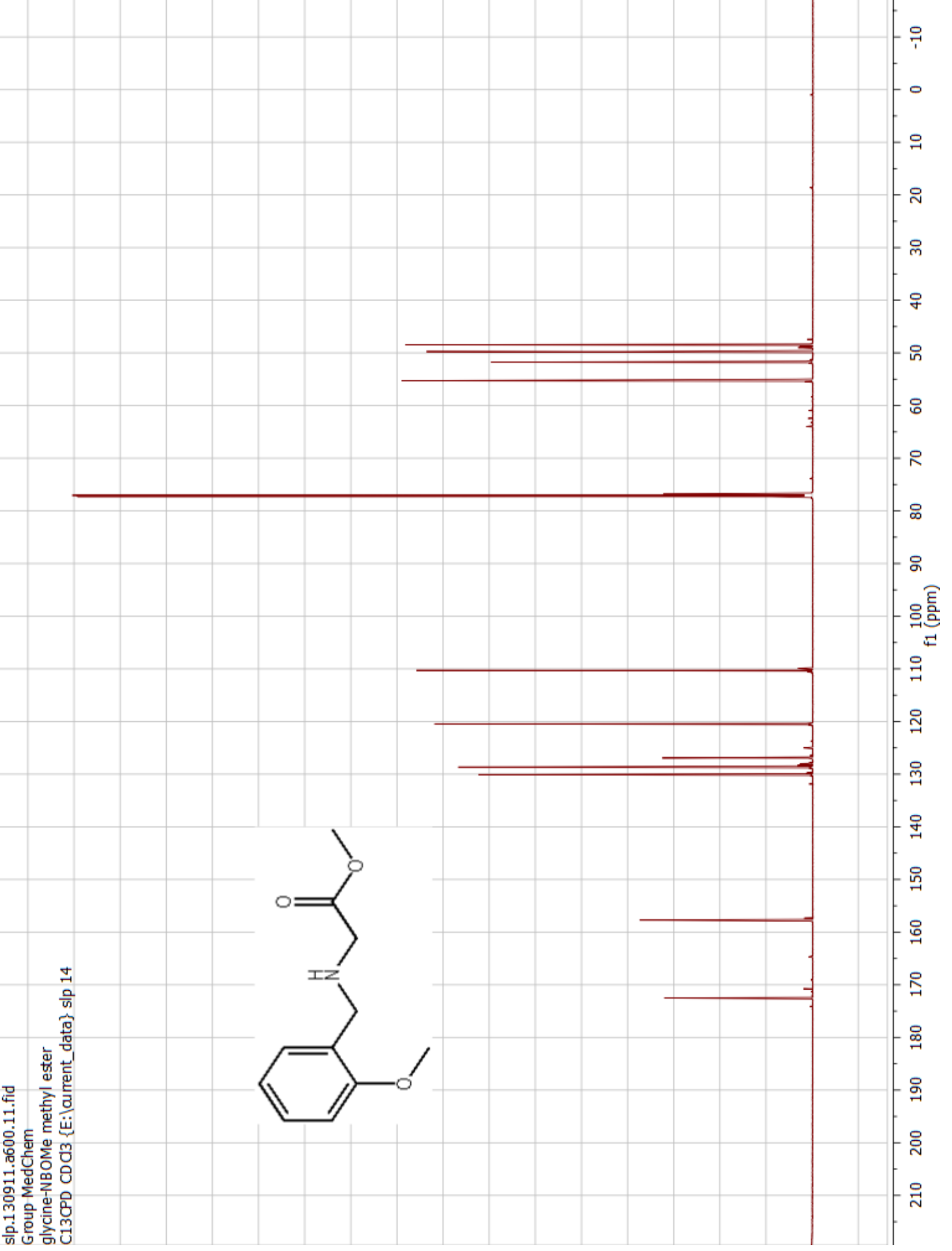




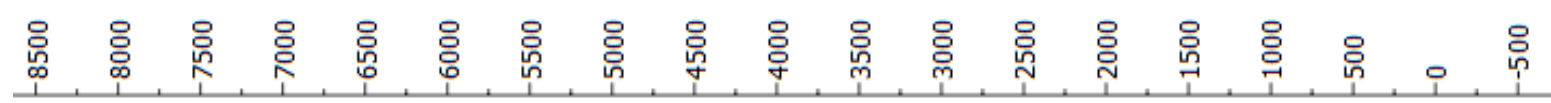

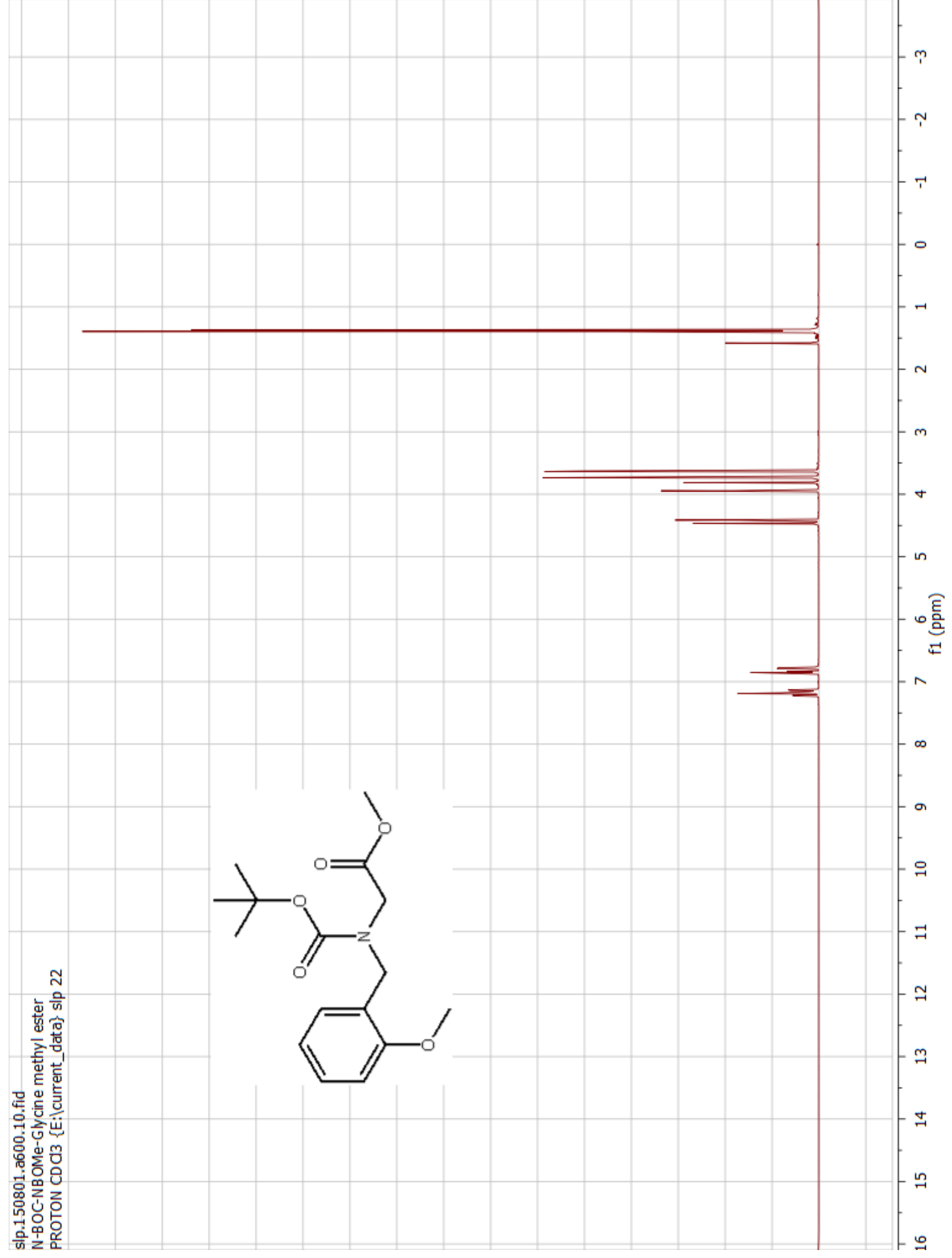




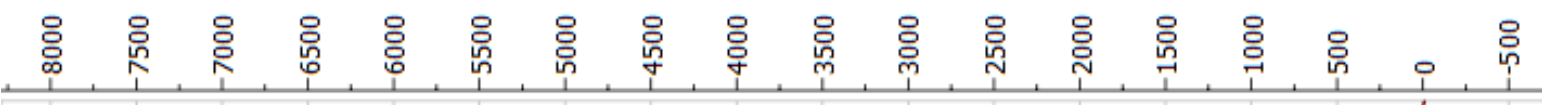

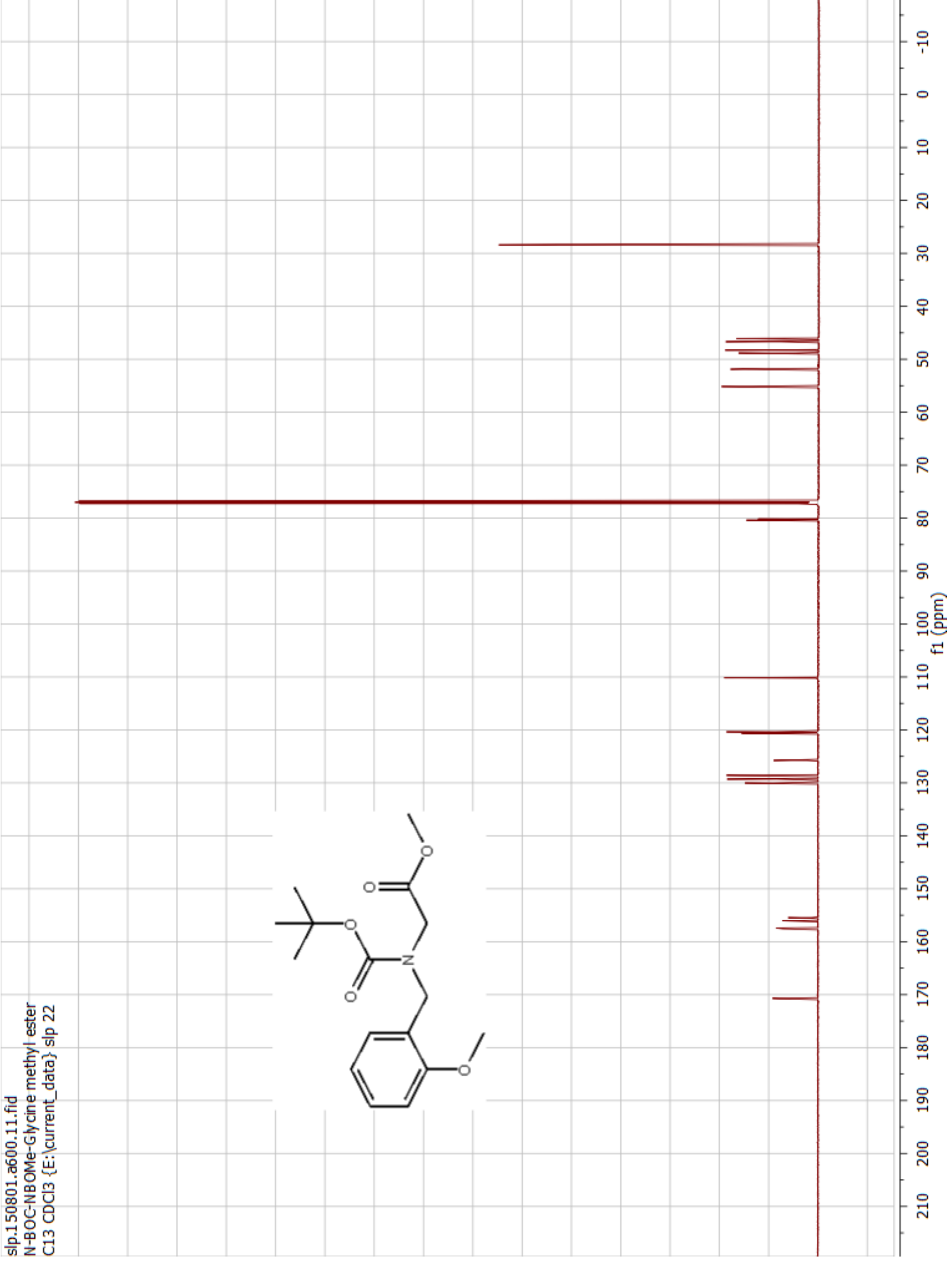




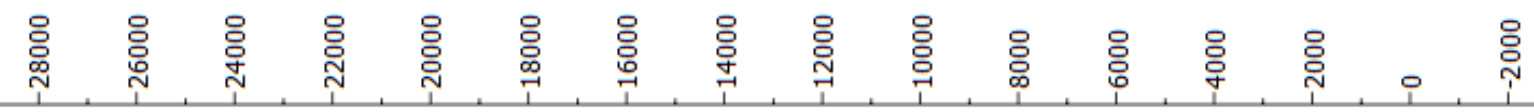

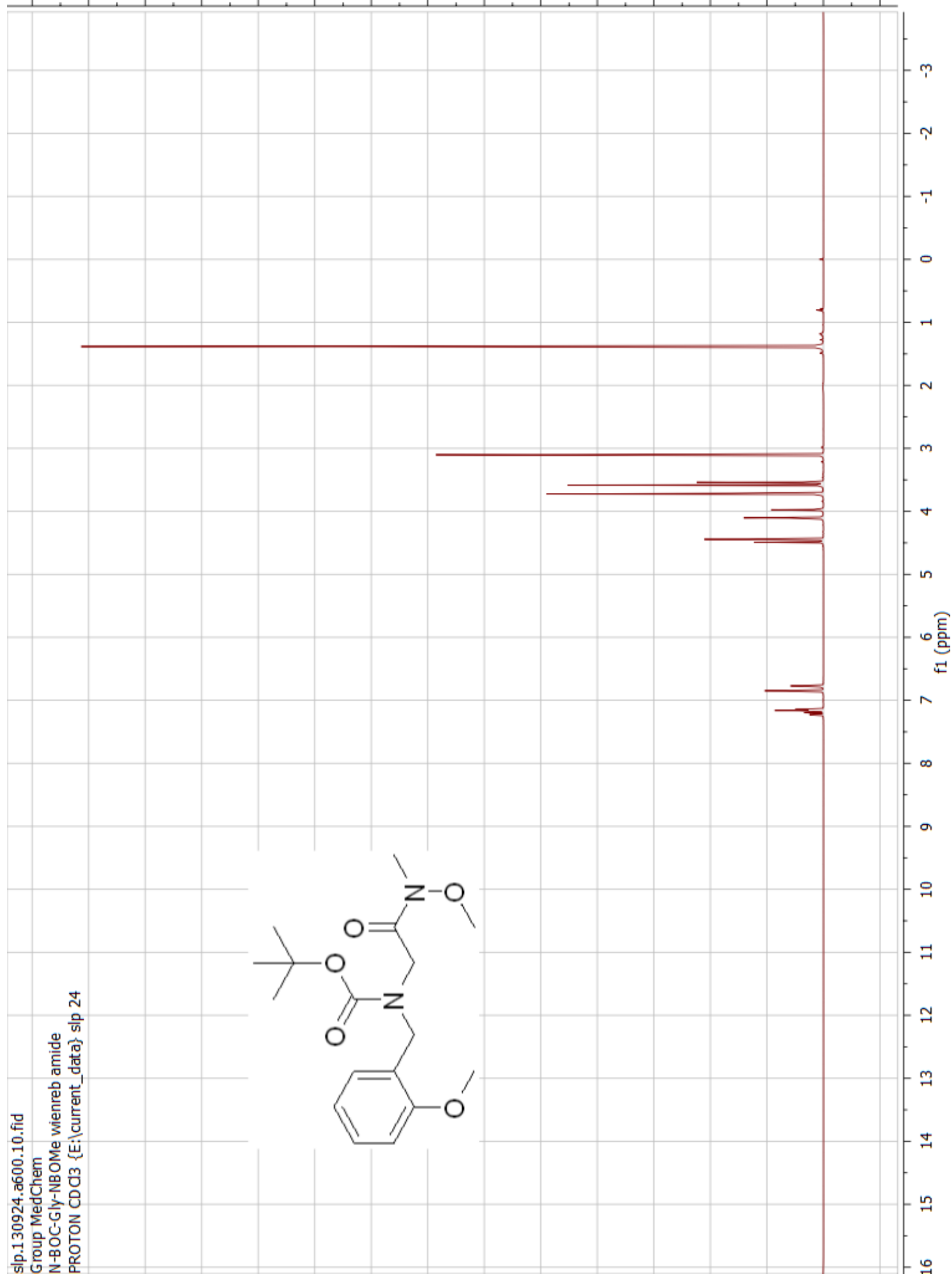




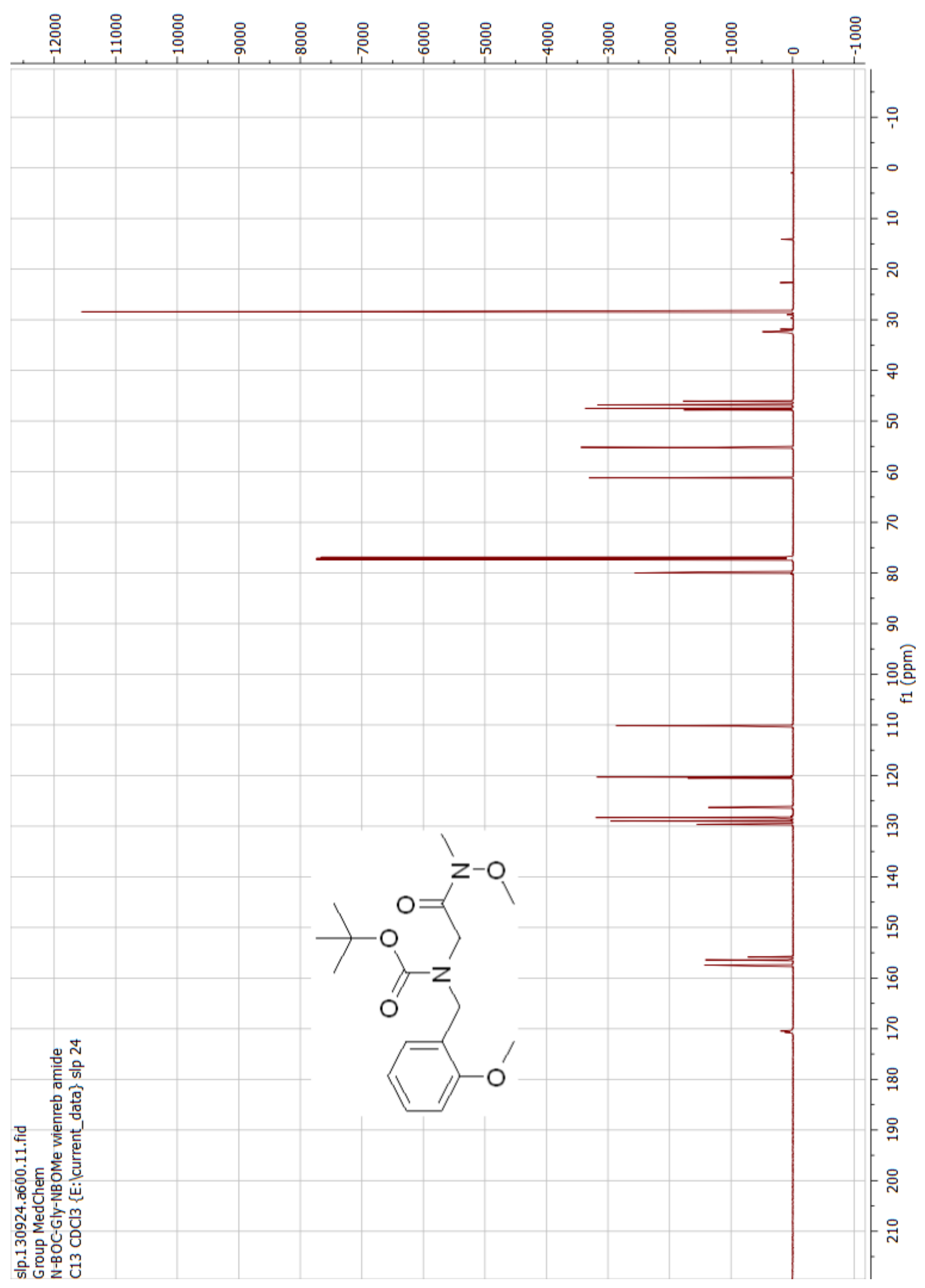




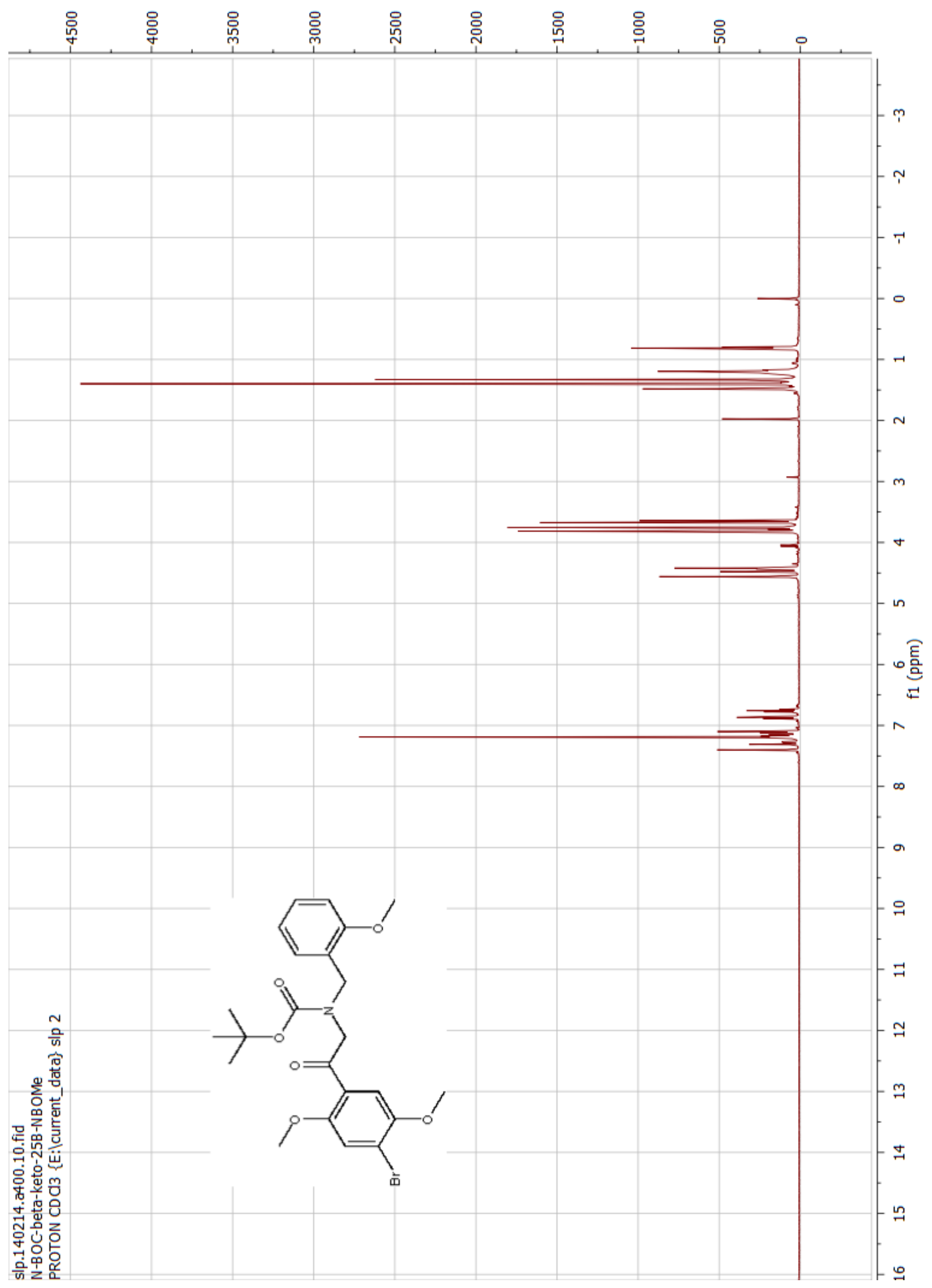




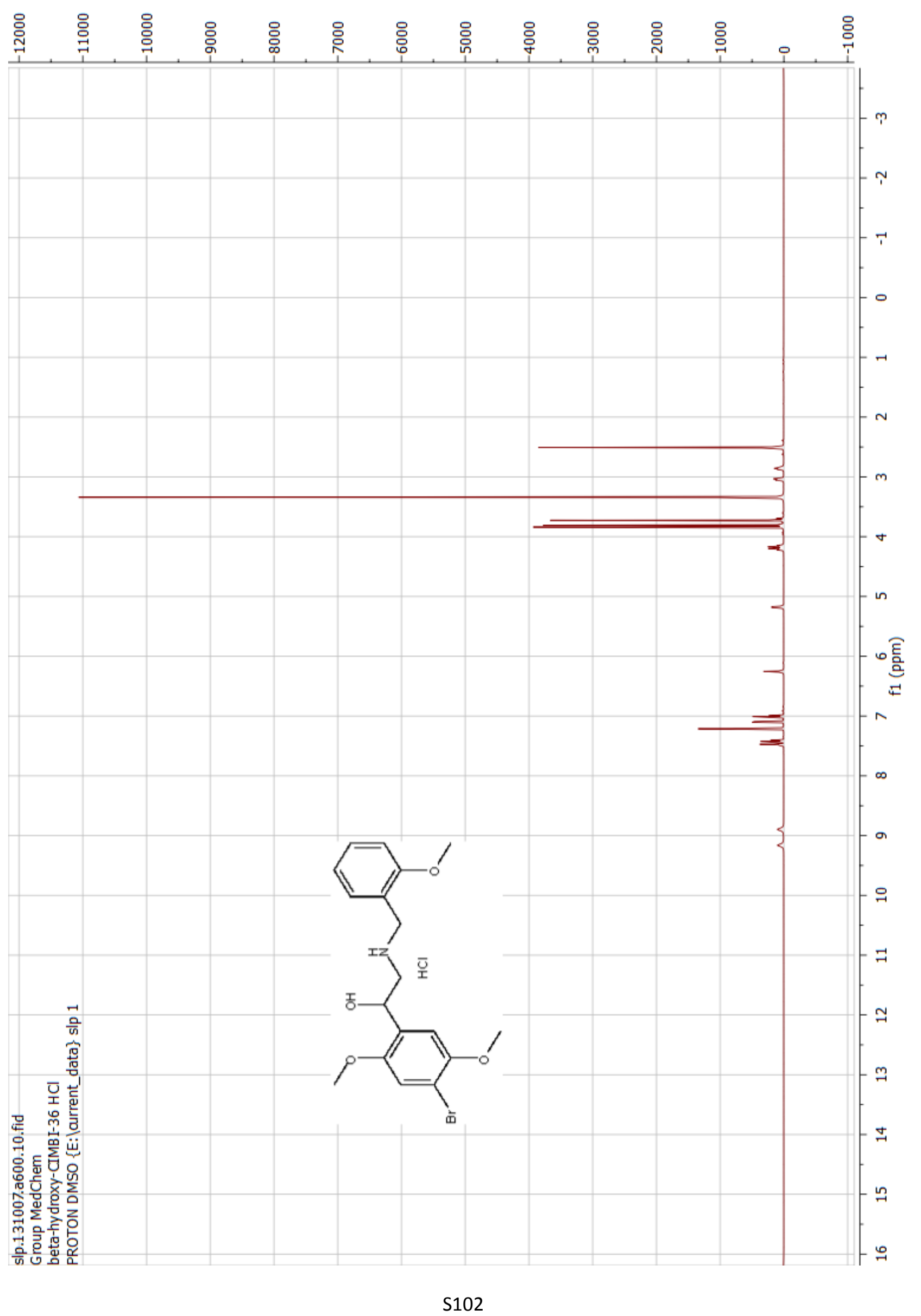




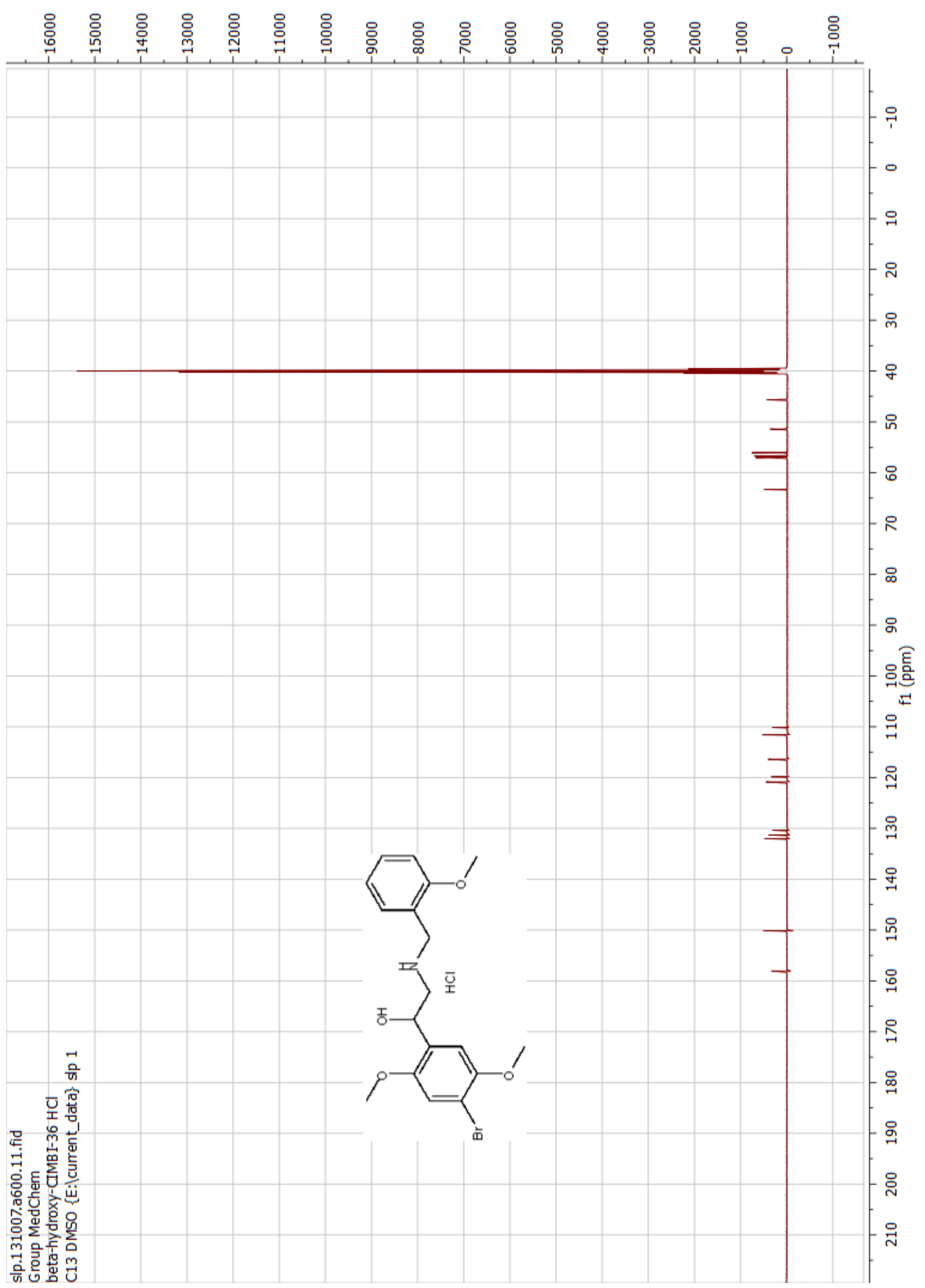




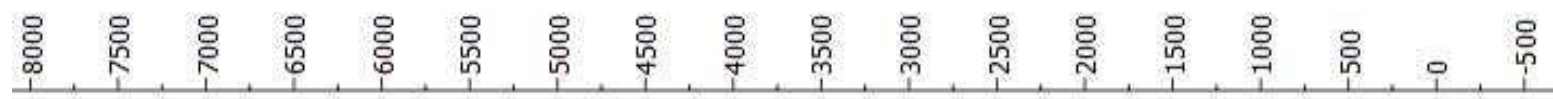

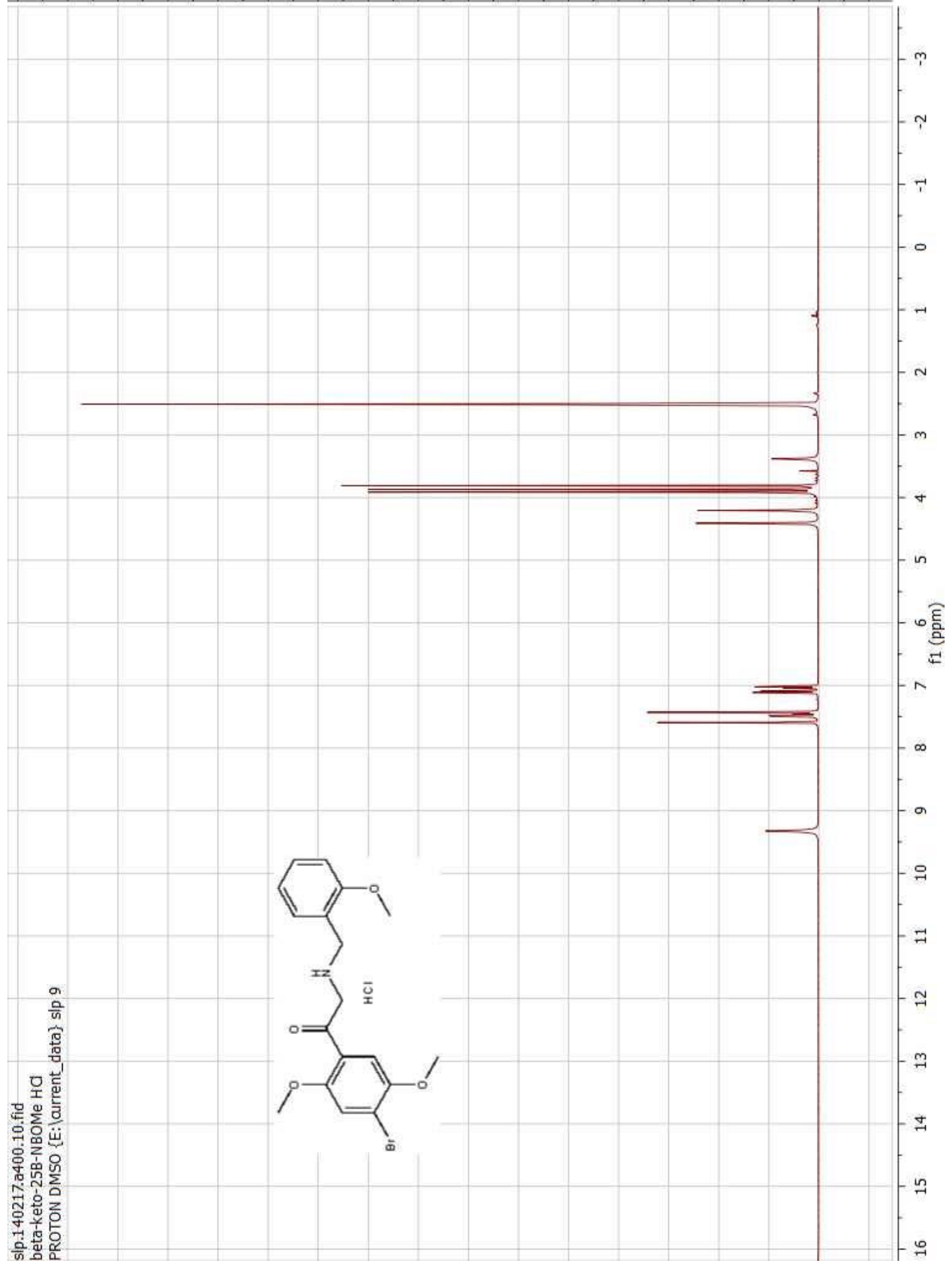




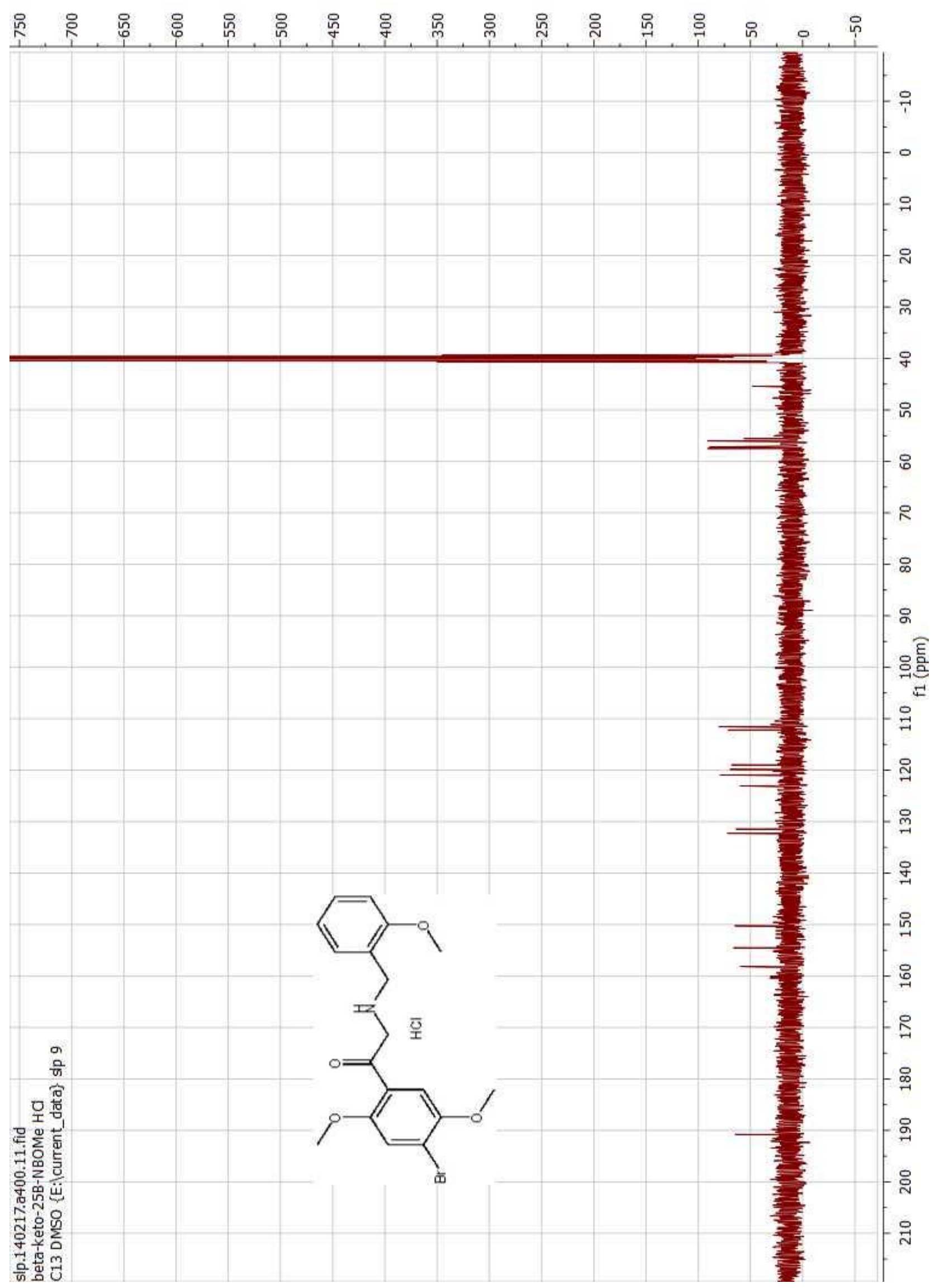




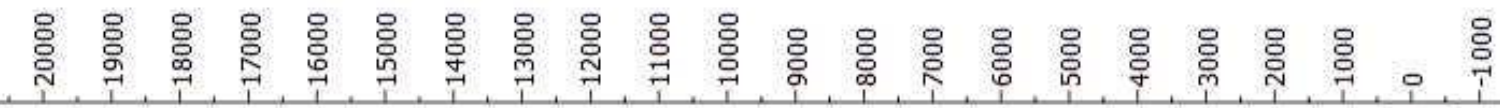

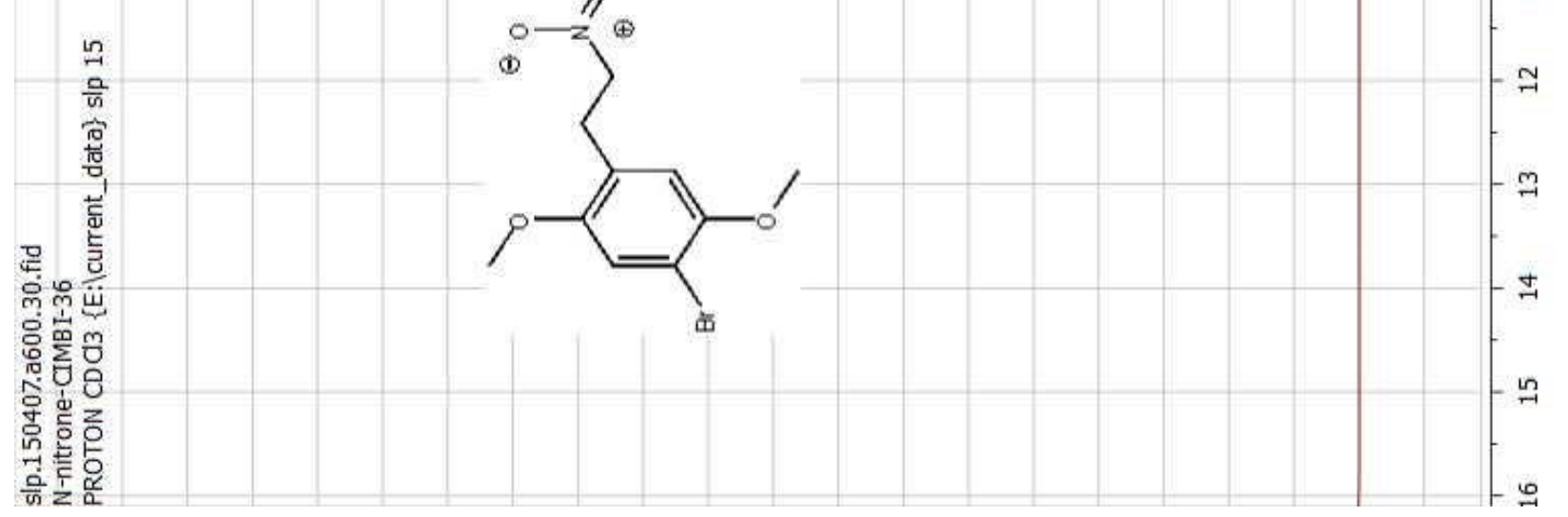




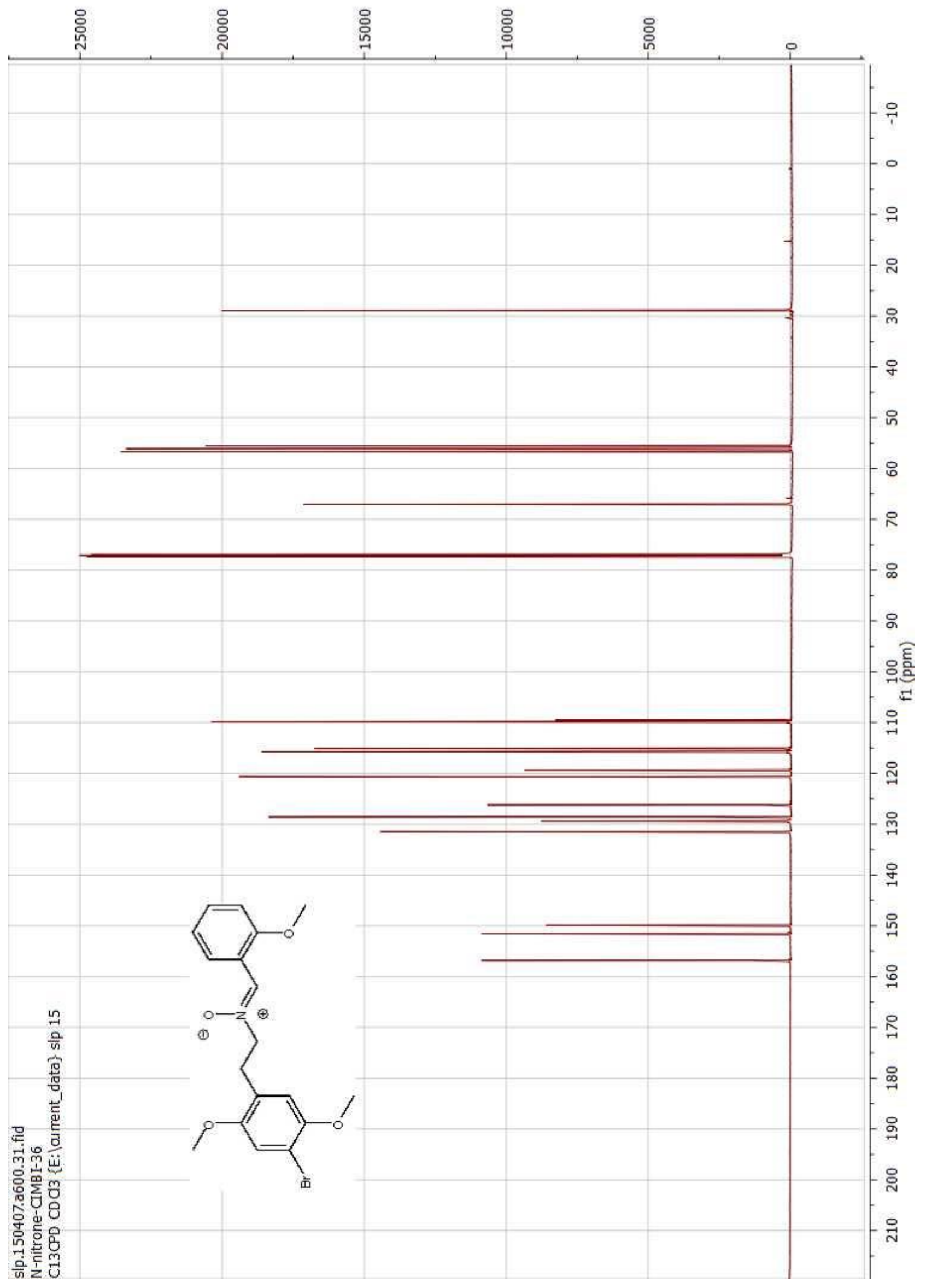




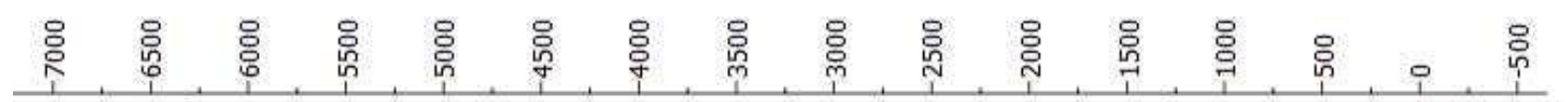

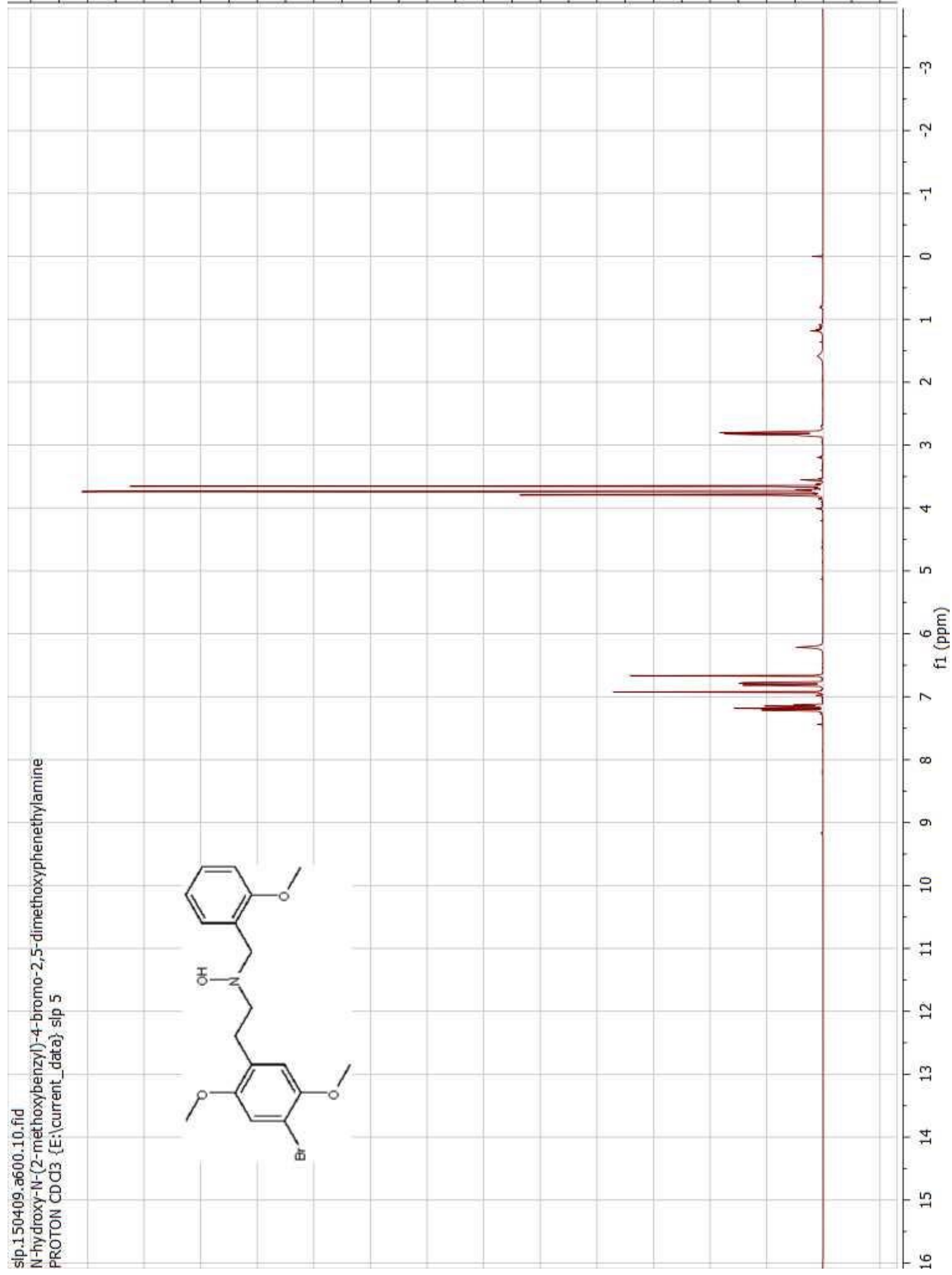




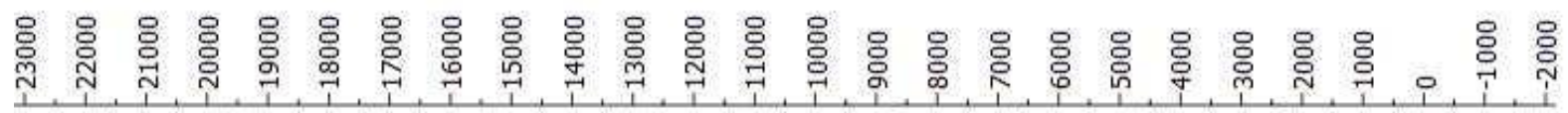

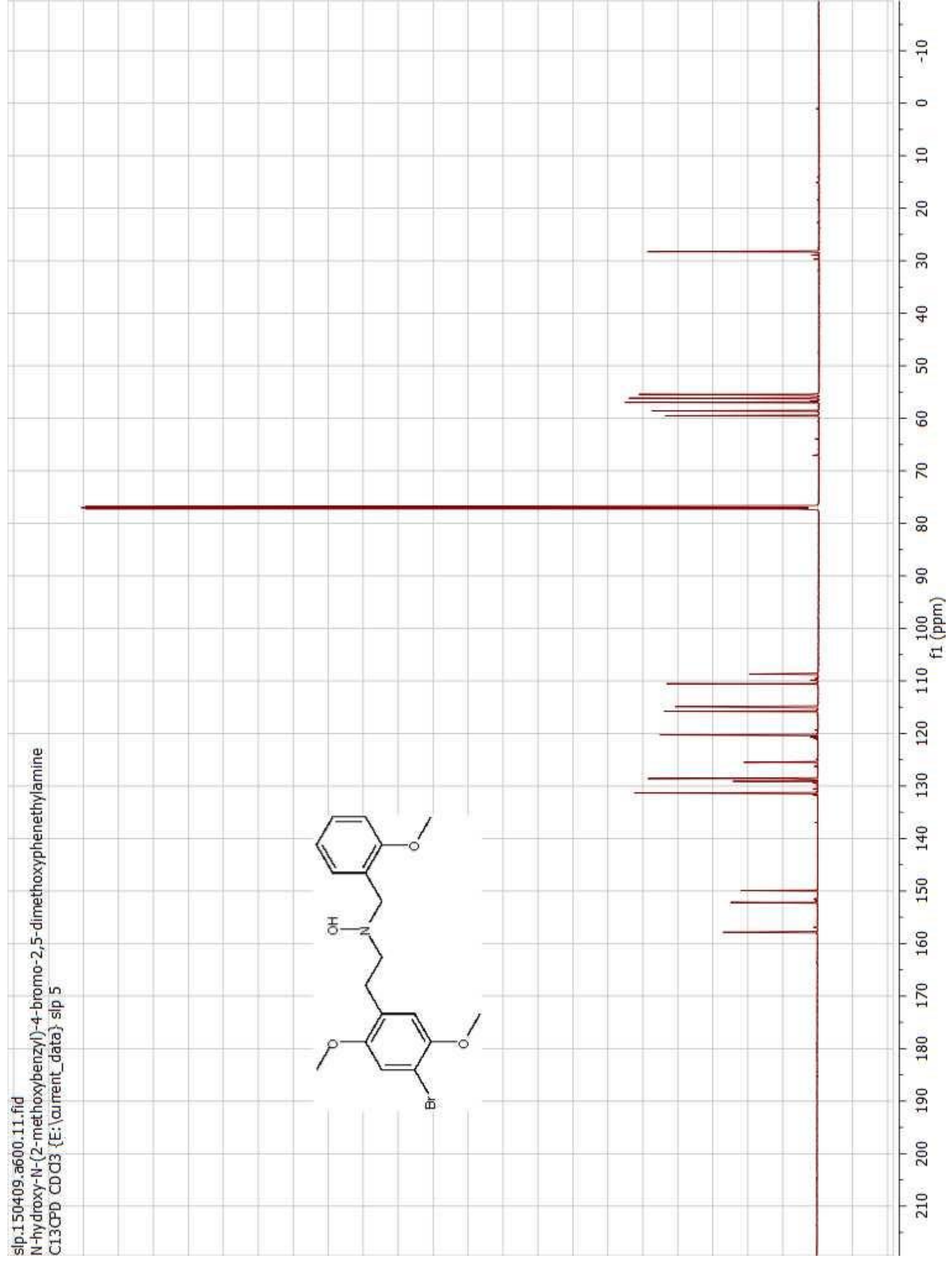




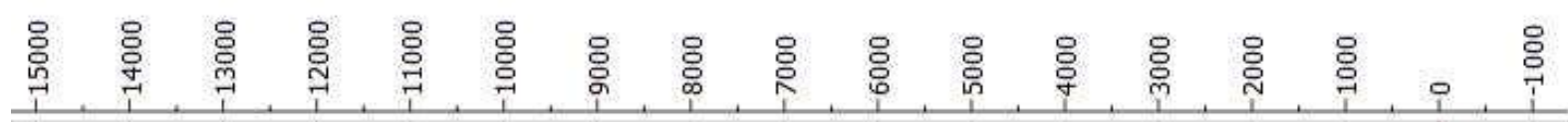

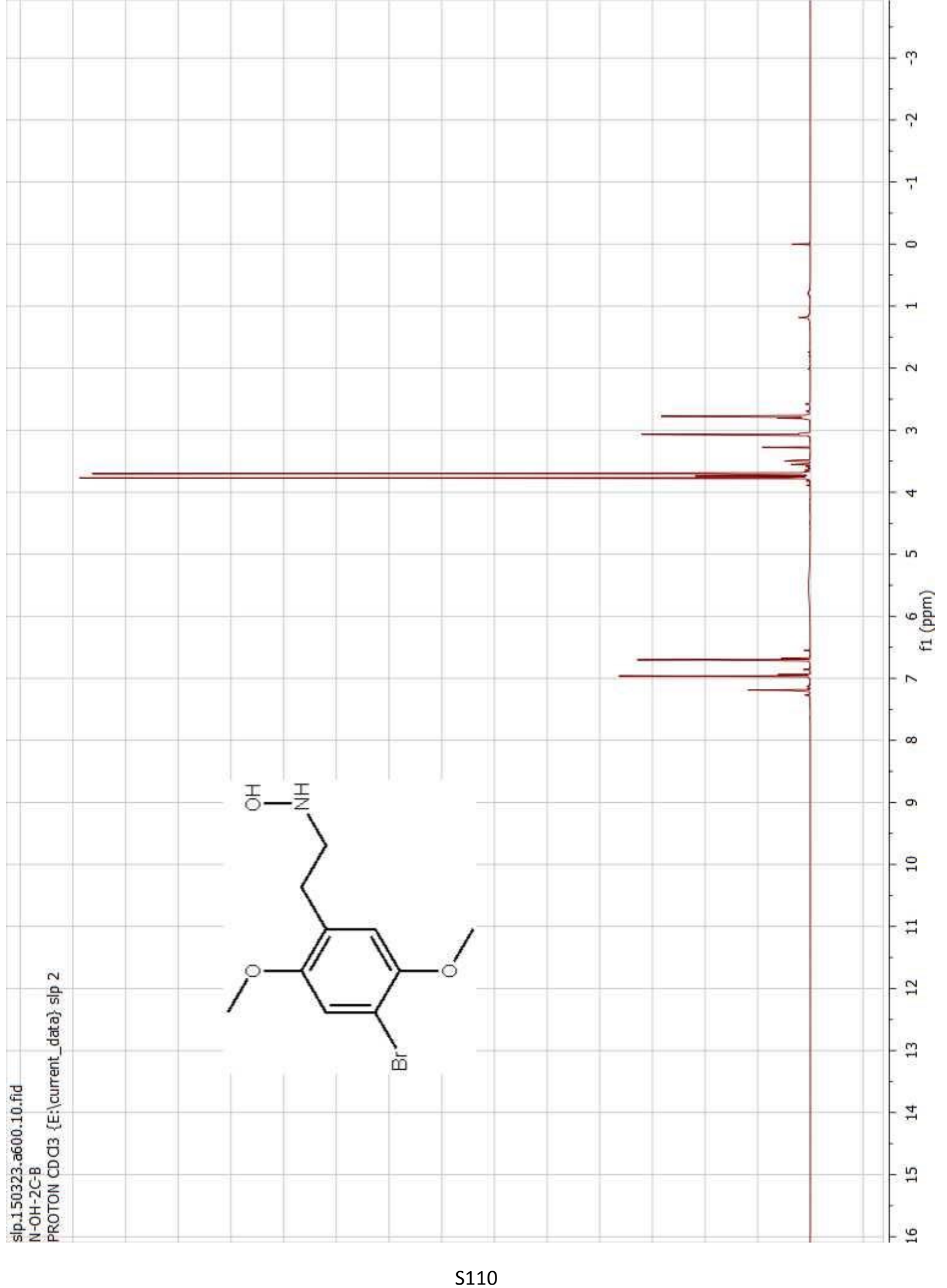




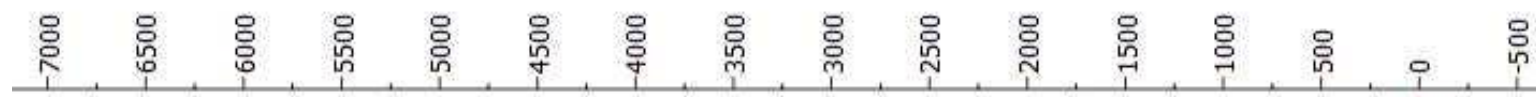

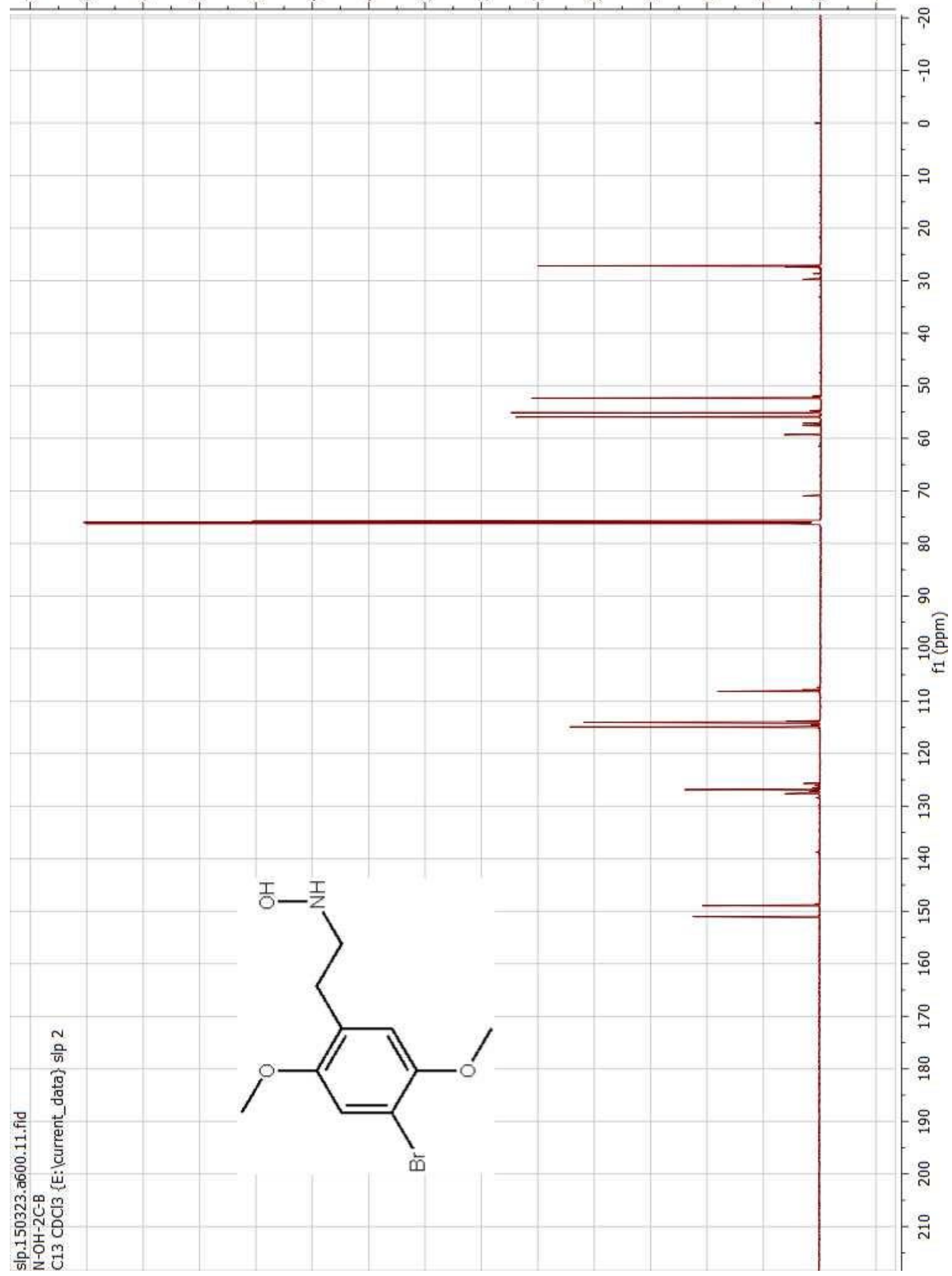




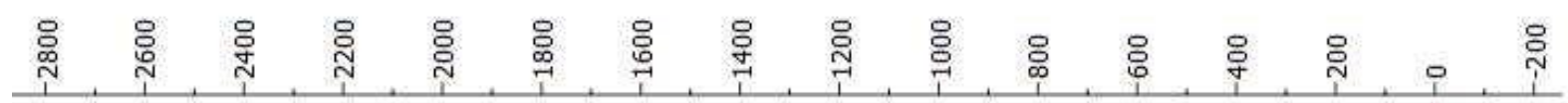

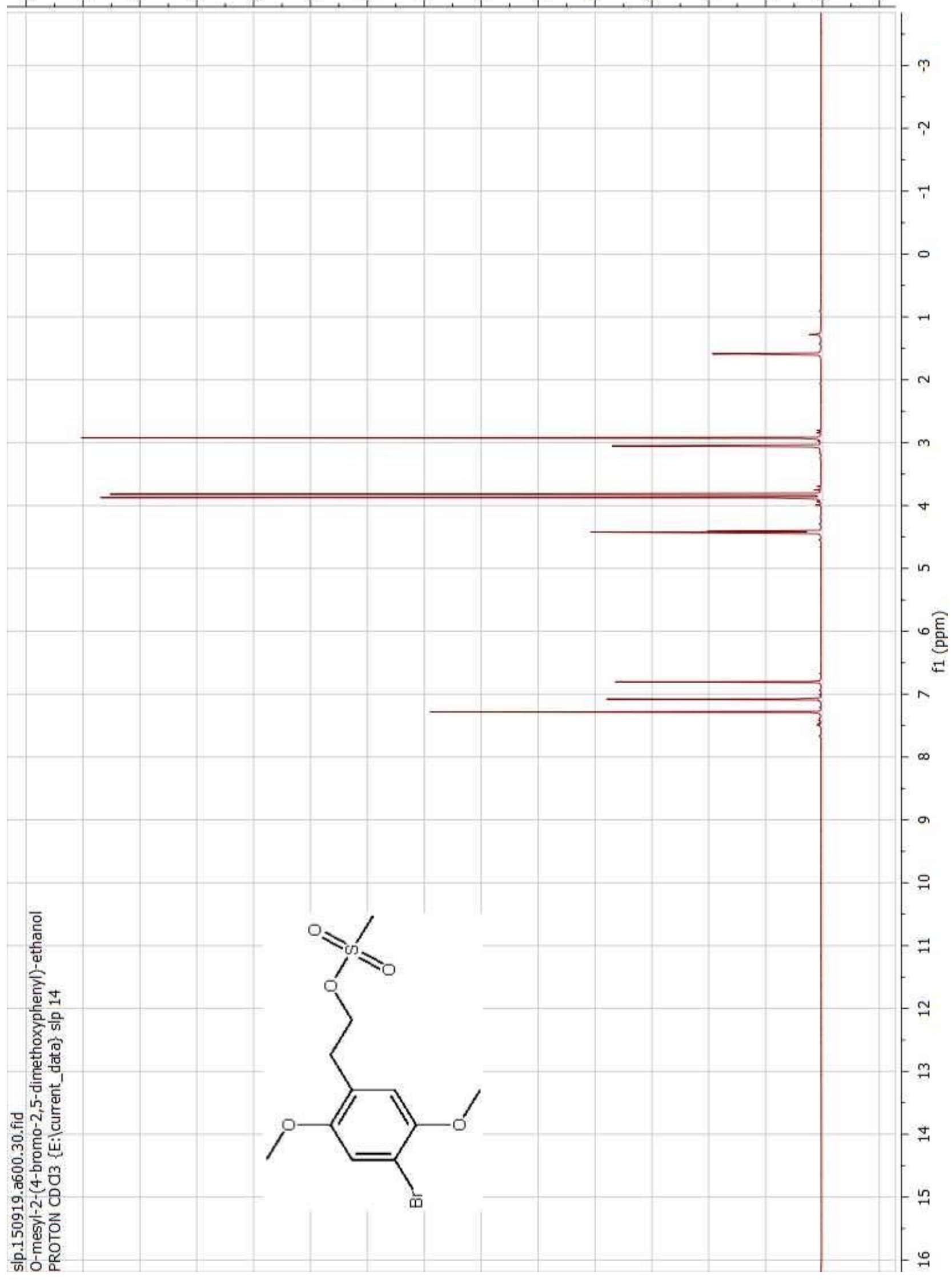




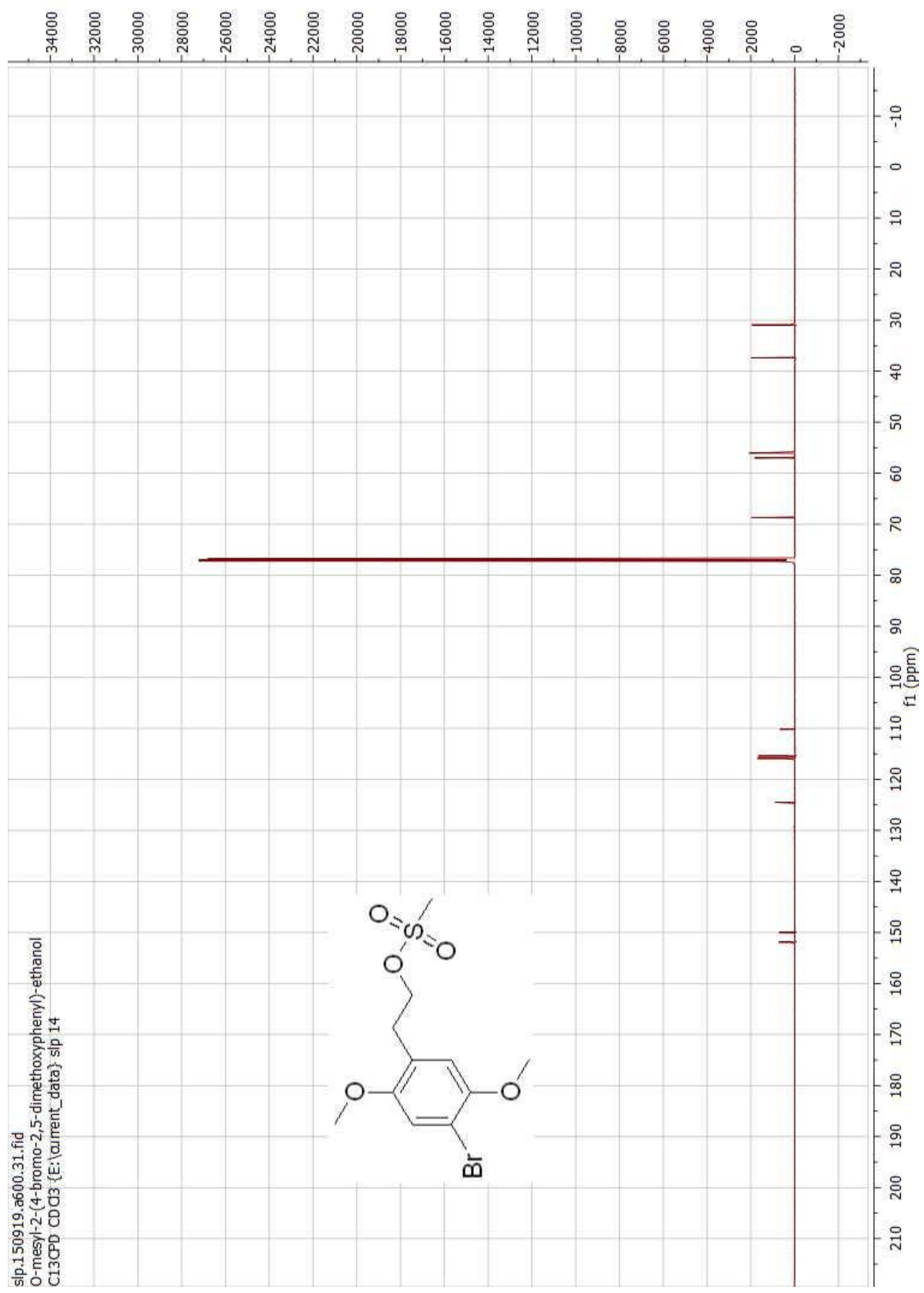




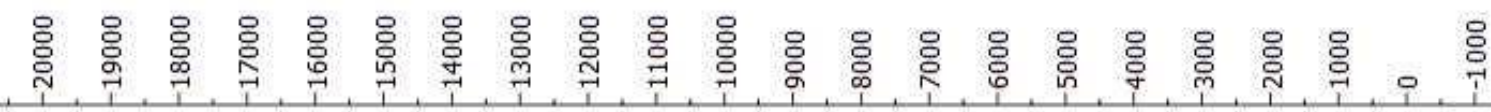

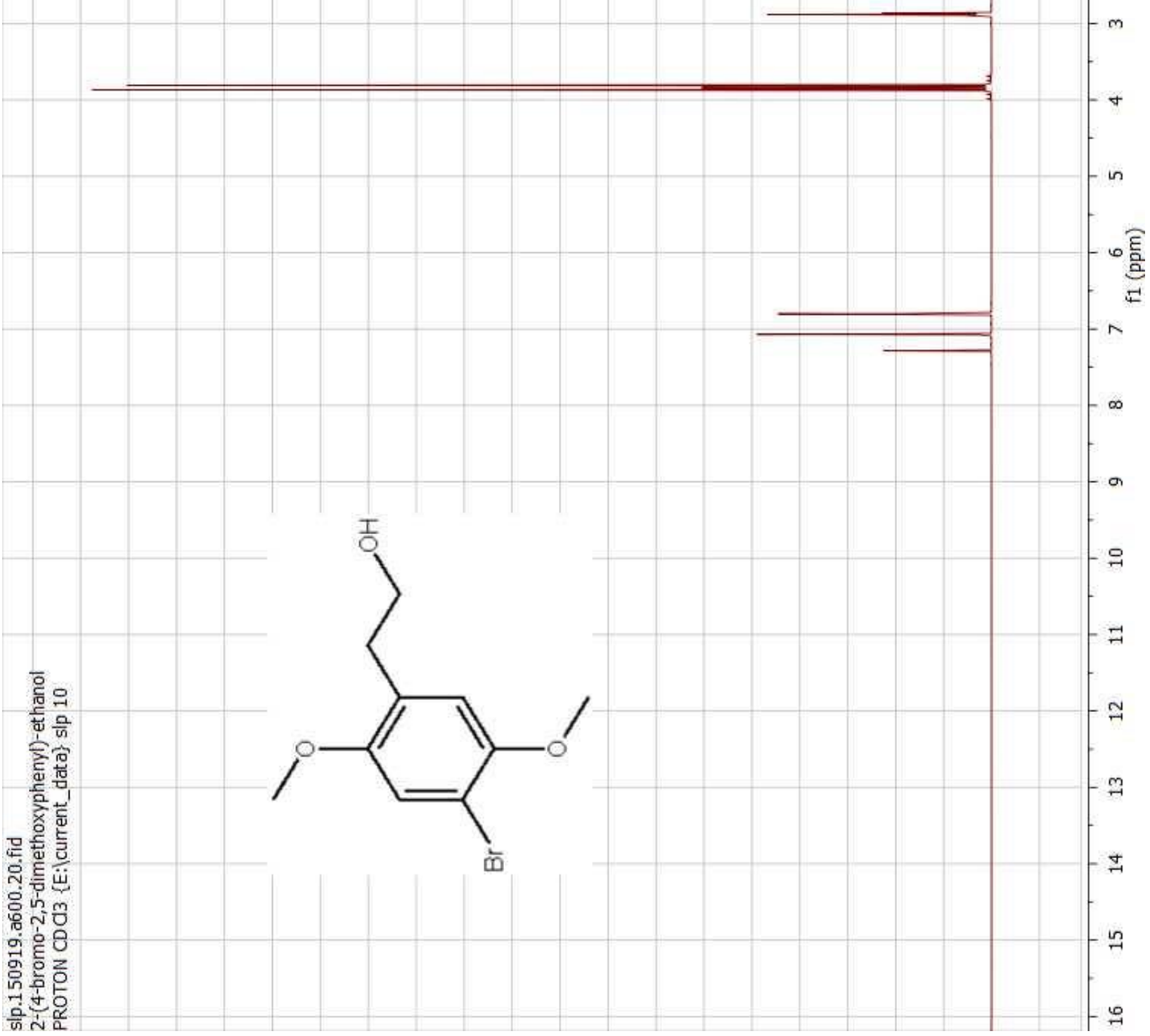




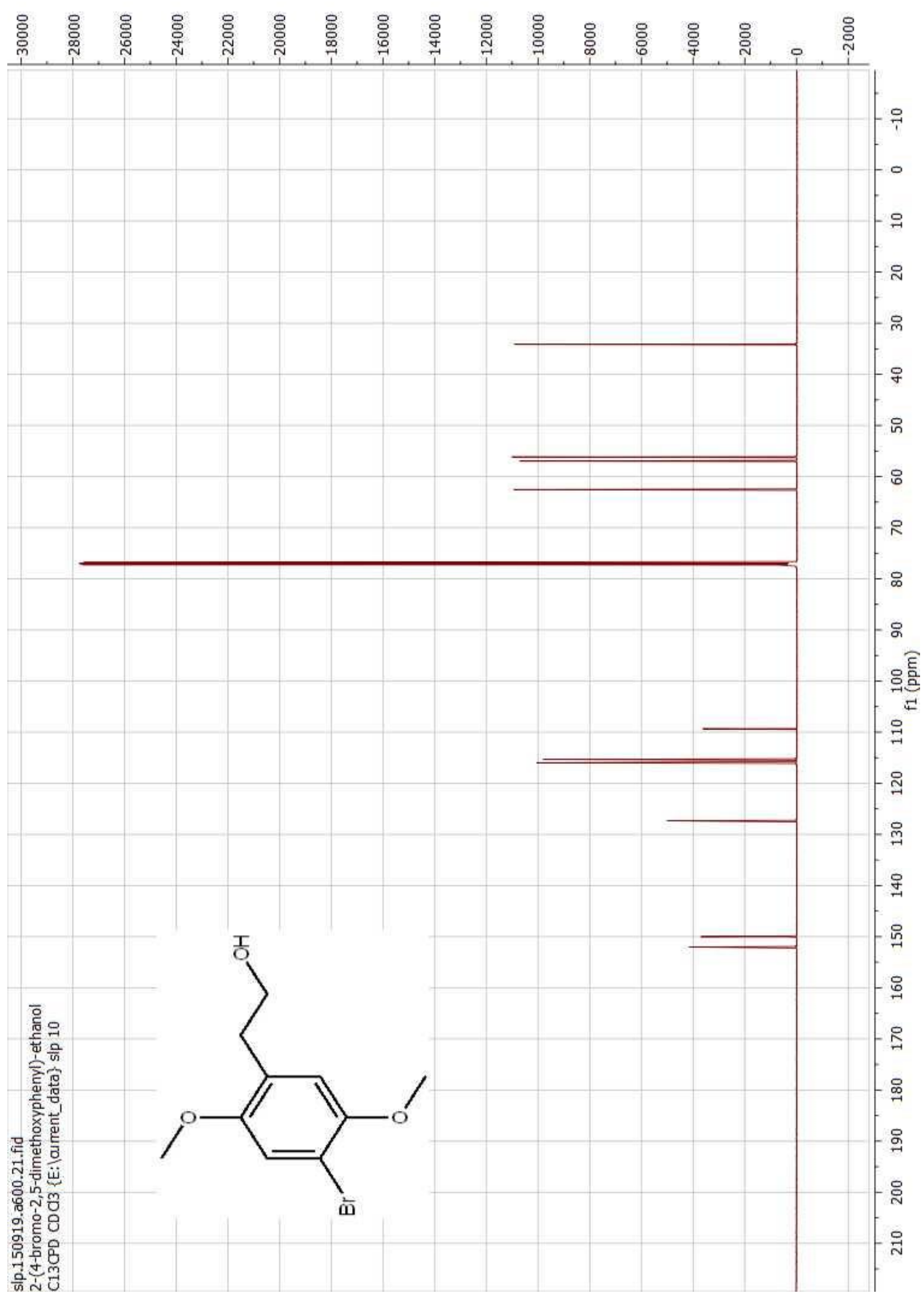




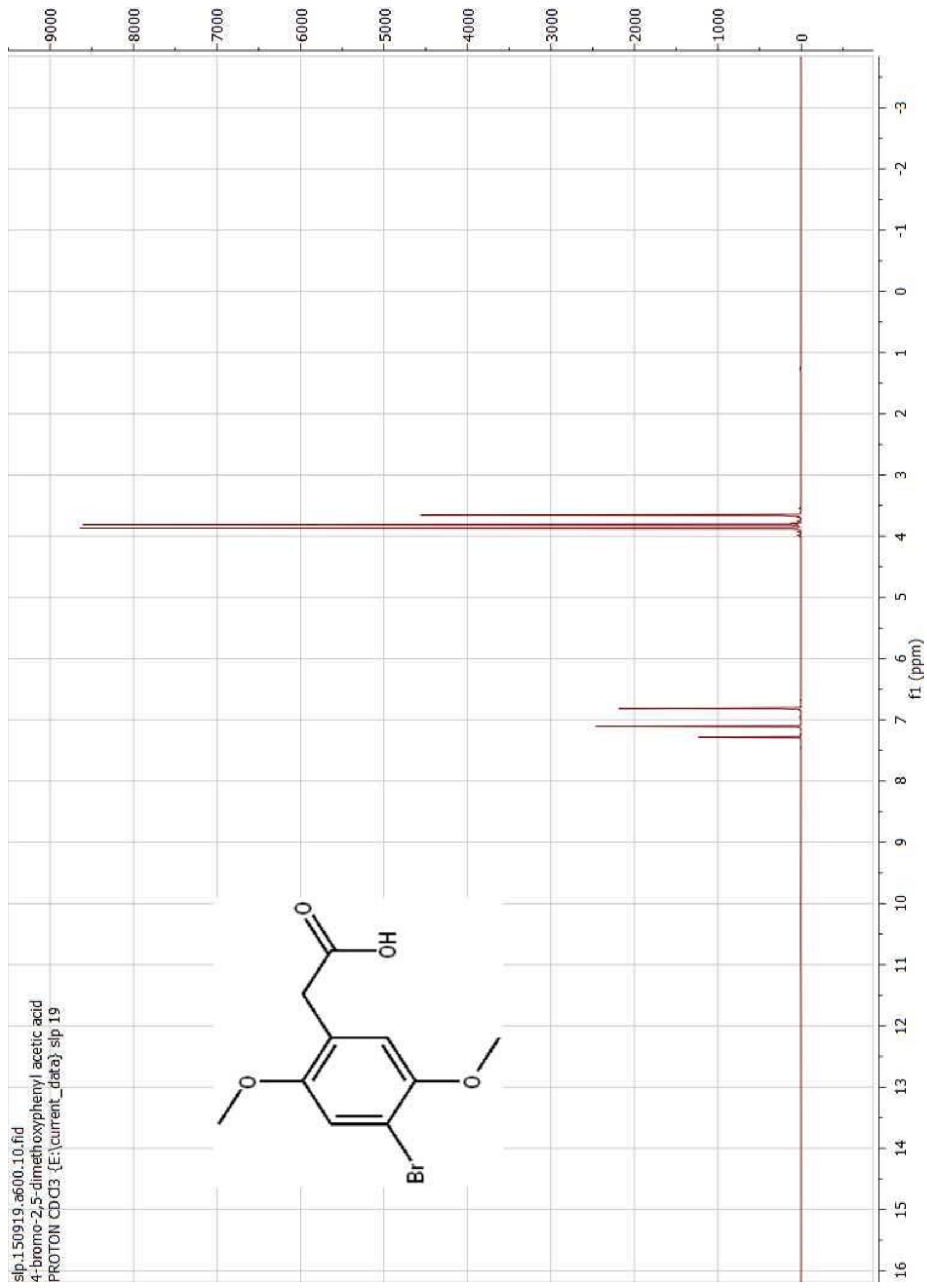




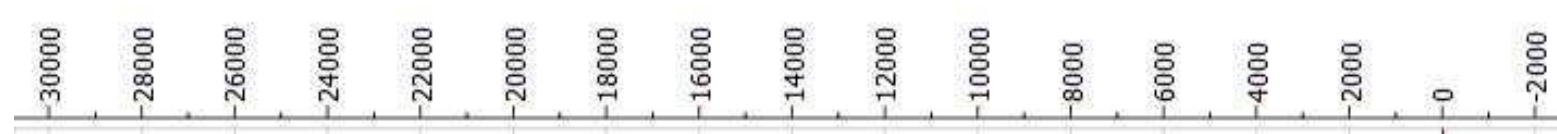

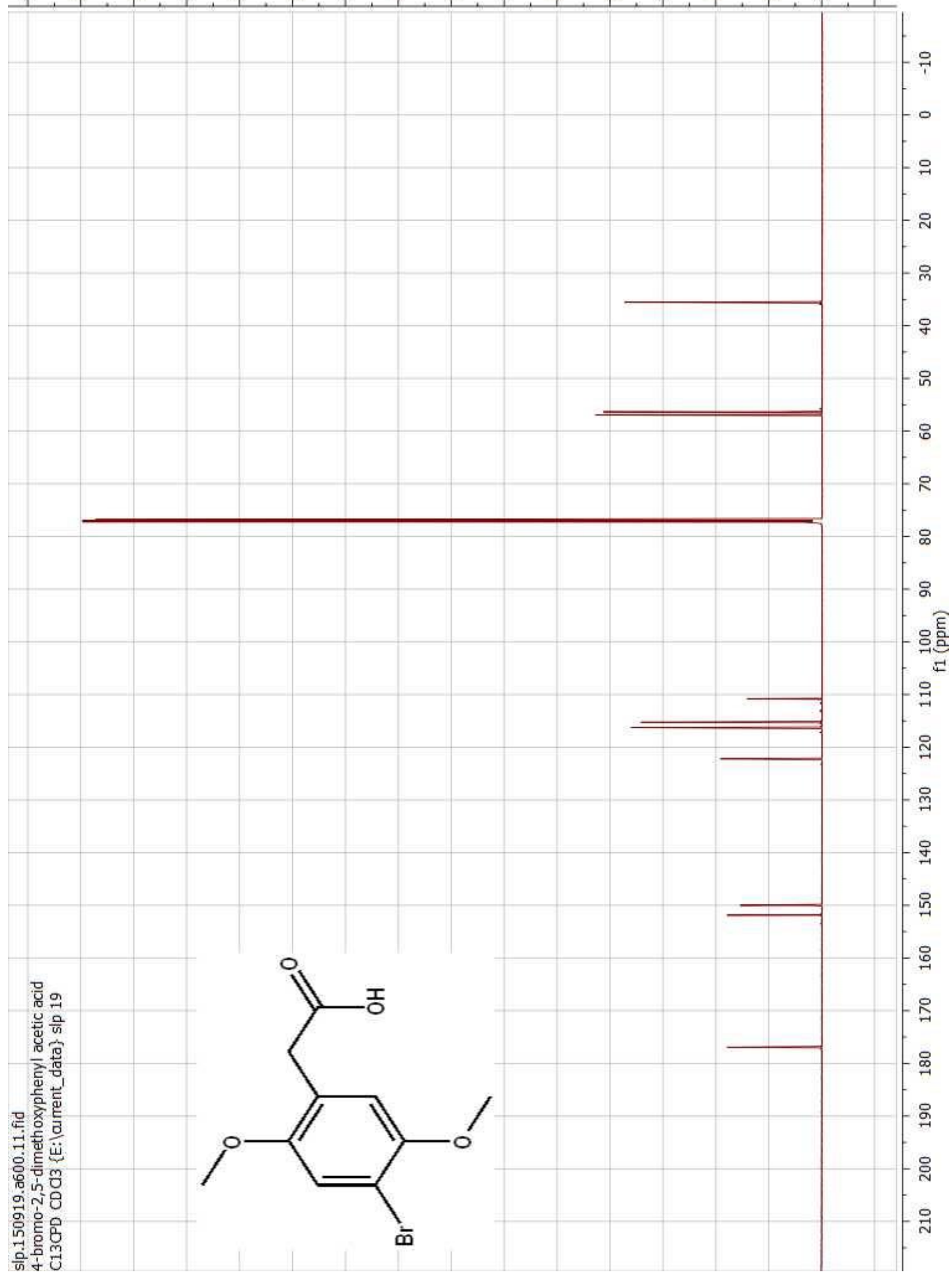

